\title{
Thermotropic polyesters and polylactide
}

Citation for published version (APA):

de Kort, G. W. (2021). Thermotropic polyesters and polylactide: a route to sustainable and reprocessable reinforced composites. [Doctoral Thesis, Maastricht University]. Maastricht University. https://doi.org/10.26481/dis.20210125gk

Document status and date:

Published: 01/01/2021

DOI:

10.26481/dis.20210125gk

Document Version:

Publisher's PDF, also known as Version of record

\section{Please check the document version of this publication:}

- A submitted manuscript is the version of the article upon submission and before peer-review. There can be important differences between the submitted version and the official published version of record.

People interested in the research are advised to contact the author for the final version of the publication, or visit the DOI to the publisher's website.

- The final author version and the galley proof are versions of the publication after peer review.

- The final published version features the final layout of the paper including the volume, issue and page numbers.

Link to publication

\footnotetext{
General rights rights.

- You may freely distribute the URL identifying the publication in the public portal. please follow below link for the End User Agreement:

www.umlib.nl/taverne-license

Take down policy

If you believe that this document breaches copyright please contact us at:

repository@maastrichtuniversity.nl

providing details and we will investigate your claim.
}

Copyright and moral rights for the publications made accessible in the public portal are retained by the authors and/or other copyright owners and it is a condition of accessing publications that users recognise and abide by the legal requirements associated with these

- Users may download and print one copy of any publication from the public portal for the purpose of private study or research.

- You may not further distribute the material or use it for any profit-making activity or commercial gain

If the publication is distributed under the terms of Article $25 \mathrm{fa}$ of the Dutch Copyright Act, indicated by the "Taverne" license above, 


\section{Thermotropic polyesters and polylactide:}

a route to sustainable and reprocessable reinforced composites 
Thermotropic polyesters and polylactide: a route to sustainable and reprocessable reinforced composites

G.W. de Kort, Maastricht University, 2021

ISBN: 978-94-6423-092-5

(C) Gijs de Kort, 2021

Cover by Gijs de Kort

Design and layout by Gijs de Kort

Printed by ProefschriftMaken

This thesis has been printed on White Zero recycled paper. The paper is $100 \%$ recycled, $\mathrm{CO}_{2}$ neutral, produced from chlorine-free pulp and suitable for further recycling.

The research leading to these results has received funding by the H2020 Framework Program of the European Union under grant agreement $N^{\circ} 685614$. The work has been carried out at the Aachen Maastricht Institute for Biobased Materials.
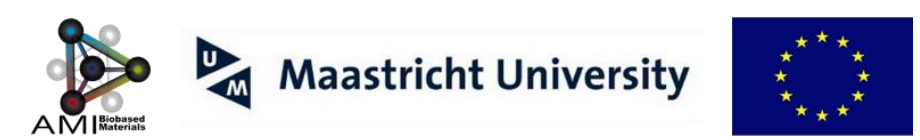


\title{
Thermotropic polyesters and polylactide: \\ a route to sustainable and reprocessable reinforced composites
}

\author{
PROEFSCHRIFT
}

ter verkrijging van de graad van doctor aan de Universiteit Maastricht, op gezag van de Rector Magnificus, Prof. dr. Rianne M. Letschert volgens het besluit van het College van Decanen, in het openbaar te verdedigen op maandag 25 januari 2021 om 13.00 uur

door

Gijsbert Willem de Kort 


\section{Promotor}

Prof. Dr. S. Rastogi

\section{Copromotor}

Dr. Ir. C.H.R.M. Wilsens

\section{Beoordelingscommissie}

Prof. dr. L. Moroni (voorzitter)

Prof. dr. A. Pich

Prof. dr. D. Vlassopoulos (Foundation for Research and Technology - Hellas)

Prof. dr. S. van der Zwaag (Delft University of Technology) 


\section{Table of contents}

Chapter 1 - Introduction $\quad 1$

1.1 Liquid crystallinity and liquid crystalline polymers 2

1.2 Thermotropic main-chain liquid crystalline polyesters 4

1.3 Thermoplastic reinforced composites 5

1.4 Sustainability and reprocessing / recycling 8

1.5 Aim and outline of this thesis. 11

1.6 References 13

Chapter 2- Effect of shear rate on the orientation and relaxation of a vanillic $\begin{array}{ll}\text { acid based liquid crystalline polymer. } & 17\end{array}$

2.1 Introduction 18

$\begin{array}{ll}2.2 \text { Methods and Materials } & 20\end{array}$

2.3 Results and Discussion 23

2.4 Conclusions 36

2.5 References 38

Chapter 3 - Controlling processing, morphology and mechanical performance in blends of polylactide and thermotropic polyesters. $\quad 41$

3.1 Introduction $\quad 42$

3.2 Methods and Materials $\quad 44$

3.3 Theoretical considerations 46

3.4 Results and Discussion 49

3.5 Conclusions $\quad 64$

3.6 References 66

3.7 Supporting Information $\quad 71$

Chapter 4 - Thermoplastic PLA-LCP composites: a route towards sustainable, reprocessable, and recyclable reinforced materials. $\quad 75$

4.1 Introduction $\quad 76$

$\begin{array}{ll}\text { 4.2 Methods and Materials } & 77\end{array}$

4.3 Results and Discussion $\quad 79$

$\begin{array}{ll}4.4 \text { Conclusions } & 87\end{array}$ 
4.5 References $\quad 88$

4.6 Supporting Information $\quad 91$

Chapter 5 - A reactive processing route to thermotropic polyesters with low processing temperature and enhanced relaxation time.

5.1 Introduction

5.2 Methods and Materials 100

5.3 Results and Discussion 103

5.4 Conclusions 117

5.5 References 118

5.6 Supporting Information 122

Chapter 6 - The importance of viscosity control for recyclable reinforced thermoplastic composites.

6.1 Introduction 130

6.2 Methods and Materials

6.3 Results and Discussion 134

6.4 Conclusions 149

6.5 References 151

6.6 Supporting Information 154

Chapter 7 - Valorization addendum

7.1 Potential and challenges regarding industrial implementation of LCP reinforced thermoplastic composites

7.2 Room for improvement: backbone composition and prepolymer molar mass

7.3 Towards different applications: cholesteric prepolymers 163

$\begin{array}{ll}7.4 \text { Closing remarks } & 165\end{array}$

$\begin{array}{ll}\text { Summary } & 166\end{array}$

$\begin{array}{ll}\text { Acknowledgements } & 170\end{array}$

$\begin{array}{ll}\text { Curriculum Vitae } & 172\end{array}$

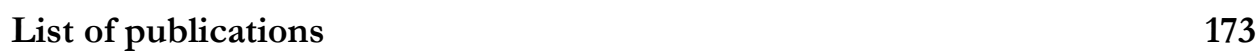


Chapter 1 - Introduction 


\subsection{Liquid crystallinity and liquid crystalline polymers}

Liquid crystalline materials can adopt a state in between the ordered, immobile crystalline state and the random, mobile liquid state. The liquid crystalline state can effectively be described as a mesophase, where the molecules are mobile but some degree of order is maintained. These materials exhibit their liquid crystalline phase under specific conditions, eg. in solution (lyotropic), or upon reaching a certain temperature (thermotropic). Different liquid crystalline phases are distinguished by their distinct molecular ordering ${ }^{1,2}$. Several distinct liquid crystalline phases have been discovered. In the nematic phase (Figure 1.1a), there is only directional ordering of the molecular axis. In smectic phases (Figure 1.1a), there is positional ordering in addition to directional ordering, as the molecules form a layered structure. The cholesteric phase (Figure 1.1b), or chiral nematic phase, resembles the nematic phase but the direction of ordering in different layers rotates due to the presence of chiral moieties.

(a)

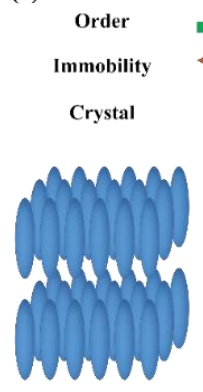

3D order

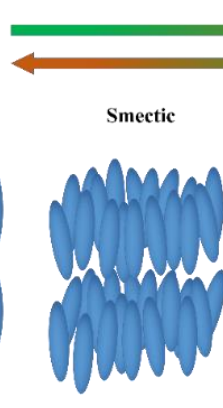

2D order

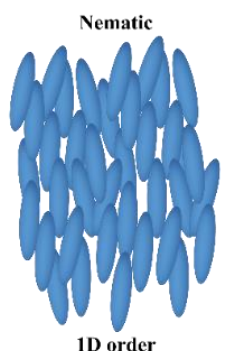

$1 D$ order (b)

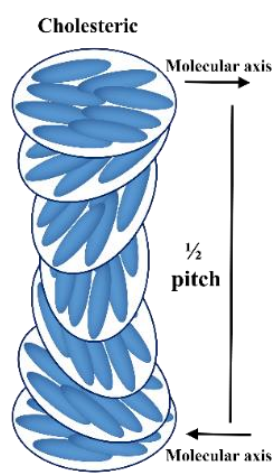

Figure 1.1 (a) Scheme showing the ordered structures of different liquid crystalline phases. (b) Structure of a cholesteric phase.

Mesogenic groups, rigid moieties with a distinct shape (typically containing chemical motives such as double bonds, benzene rings, or other ring structures), are present in these materials ${ }^{1,2}$. These moieties are the cause of liquid crystalline behavior, as disc- or rod-like, rigid parts of the molecules cannot interact efficiently when ordered randomly. To achieve the most favourable thermodynamic state, the molecules need to orient (decreasing the entropy of the system) to allow effective interactions (decreasing the enthalpy of the system). Upon heating, the isotropic state can be obtained. Liquid crystalline phases are observed in a wide range of materials including small molecules, polymers and colloidal systems. The observation of liquid crystalline phases in systems built up of large units such as colloids implies that steric interactions can also play a large role. Liquid crystalline polymers can be considered as a number of rigid segments, 
similar to liquid crystalline small molecules, which are connected. The behavior of a liquid crystalline polymer is strongly influenced by the way the mesogenic units are incorporated into the polymer chain, eg. as pendant groups connect via a non-mesogenic backbone (side-chain LCPs), or along the backbone of the polymer (main-chain LCPs). The LCPs described in this thesis are main-chain LCPs, of which properties, such as the nematic-isotropic transition temperature, mechanical performance, and response to external fields, is not only dependent on the chemical composition of the backbone but also on the length of the polymer chain.

Liquid crystalline polymers (LCPs) are an exceptionally versatile class of materials, as the rigid moieties can be built into the polymers in many different ways, resulting in vastly different properties. For example, LCPs can be used as optical materials and responsive materials (liquid crystalline elastomers) ${ }^{3,4}$, as exceptionally strong materials (highly oriented LCP fibers) $)^{5,6}$, or as materials with incredible thermal resistance (liquid crystalline thermosets) $)^{7,8}$.
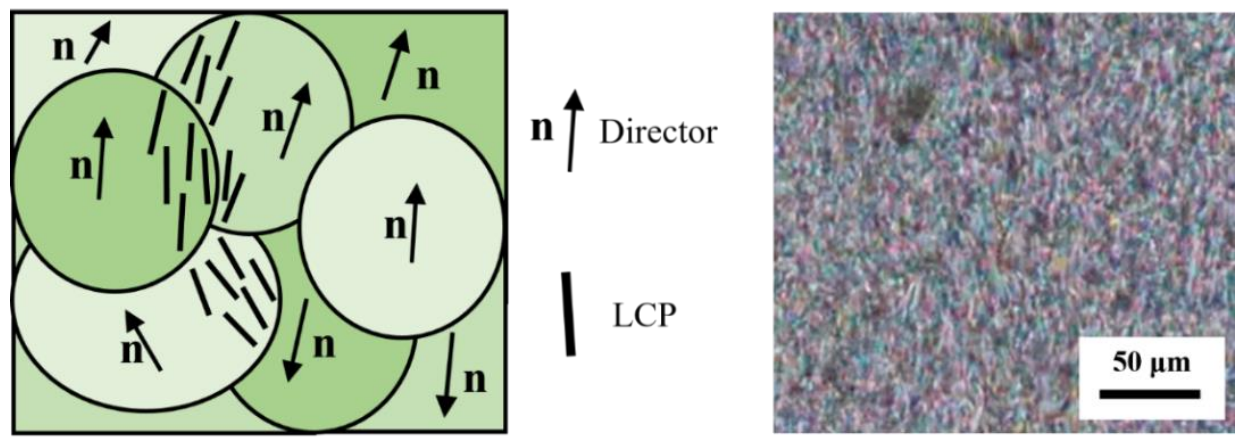

Figure 1.2 Schematic representation of the polydomain texture as observed in nematic LCPs and an example as viewed between crossed polarizers.

The direction along which the molecular axis of a nematic material is oriented is not necessarily constant on a macroscopic level: regions with different preferred directions of alignment exist within a sample (Figure 1.2, left) ${ }^{9}$. Molecules cannot effectively move between different regions, as they are mainly mobile along the direction of the (shared) molecular axis. This structure consisting of domains with local directional ordering, separated by boundaries, is associated with the (polydomain) texture of a liquid crystalline material as is observed between crossed polarizers by optical microscopy. Since liquid crystalline materials are inherently birefringent, due to their anisotropic nature, optical microscopy between crossed polarizers unravels the morphological characteristics of the liquid-crystalline phase. A schematic representation of the polydomain texture of nematic LCPs and an example of the Schlieren texture that is 
typically observed in nematic phases are shown in Figure 1.2. The polydomain texture of liquid crystalline materials contributes to the complexity of their viscoelastic behavior.

\subsection{Thermotropic main-chain liquid crystalline polyesters}

A class of LCP that is of great relevance for this thesis are thermotropic main-chain LCPs, and more specifically the polyesters of this class. These materials exhibit a liquid crystalline phase above a certain temperature and the rigid moieties are incorporated into the backbone of the polymeric chains. Generally, thermotropic LCPs have a high persistence length compared to typical thermoplastic polymers, and respond to flow in a unique way ${ }^{9-11}$. This makes for materials that are melt processable, with a relatively low viscosity, and that are easily oriented in a flow field. Upon deformation, the polydomain texture is stretched and the domains are subsequently broken up. During these processes, the LCP chains align parallel to the flow direction on a macroscopic level. These flow properties are beneficial as they allow for the facile production of strong objects with coherently oriented chains on a macroscopic level via common melt processing techniques such as fiber-spinning 5,12 , injection molding ${ }^{13,14}$, or even 3D printing ${ }^{15}$. The high cooling rates that the material experiences in these processing methods are crucial to maintain a high level of chain-orientation in the processed LCPs.

Thermotropic polyesters are produced via a melt-polycondensation of aromatic monomers derived from dicarboxylic acids, diols, and hydroxyl acids. The polymerization proceeds via acidolysis ${ }^{16,17}$, where an acetylated hydroxyl group reacts with a carboxylic acid under the formation of an ester and acetic acid, as shown in Figure 1.3. Typically, the polymerization starts at $200{ }^{\circ} \mathrm{C}$ under inert atmosphere while acetic acid is removed. To promote acidolysis, the temperature is gradually increased to 300 $350{ }^{\circ} \mathrm{C}$. In the final stage of the reaction, vacuum is applied to remove the remaining acetic acid. These high reaction temperatures result in numerous side reactions ${ }^{17,18}$, but are required to suppress crystallization during polymerization. The requirement of high polymerization temperatures imposes challenges in the incorporation of rigid bio-based monomers due to their lower thermal stability. The high temperatures that are required to suppress the crystallization of these LCPs, implies that equally high temperatures are required during processing of LCPs in the nematic melt $\left(\mathrm{T}_{\text {processing }}>300^{\circ} \mathrm{C}\right)$. 

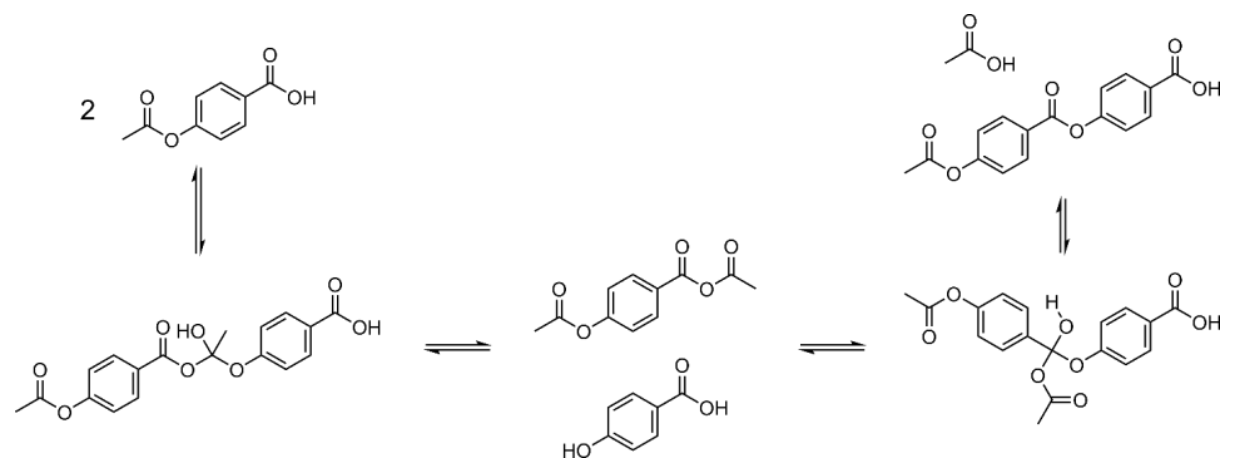

Figure 1.3 Representation of the coupling of two p-acetoxy benzoic acid molecules via acidolysis. In an acidolysis polycondensation the formed acetic acid is removed in order to push the chemical equilibria towards the formation of polymer.

The processing temperature of LCPs can be reduced by decreasing the regularity of their backbone, as this reduces the formation and melting temperature of potential crystallites ${ }^{9}$. This can be done by numerous methods ${ }^{19-22}$, such as incorporating bent monomeric units (eg. 3-hydroxy benzoic acid), incorporating substituted monomeric units (eg. vanillic acid), increasing the overall number of co-monomers, or by incorporating flexible monomeric units (eg. suberic acid).

\subsection{Thermoplastic reinforced composites}

Thermoplastic composites consist of two or more immiscible melt-processable polymer phases. During melt processing the minor phase is dispersed in the matrix and a blend is formed. The properties of the composite depend on the type of dispersed phase used (eg. a rubbery filler typically results in improved impact properties, whereas a hard filler can be used to increase the stiffness). This thesis focusses on reinforced composites: the stiffness and strength of the polymer matrix are increased by the addition of the dispersed phase, which typically has a fibrous morphology. The use of main-chain LCPs as the dispersed phase provides a facile route to obtain reinforced thermoplastic polymers ${ }^{23,24}$, as the LCP can form strong fibrils within the matrix under the right conditions (Figure 1.4, left). Fiber-reinforced composites are known for their excellent mechanical properties in combination with a low density ${ }^{25}$. The mechanical performance of LCP reinforced thermoplastic composites is comparable to that of short glass fiber-reinforced materials ${ }^{24,26}$, and is unlikely to compete with composites based on carbon fiber or pre-made fibers of lyotropic LCPs. The true potential of the LCP reinforced thermoplastic composites lies in the fact that they are highly compatible with conventional melt processing techniques (allowing cheap production and versatility in terms of design), and their potential to contribute to a circular economy. 
The latter refers to their ability to be effectively reprocessed without deterioration of their mechanical performance, which opens up a route to obtain more sustainable reinforced composites.
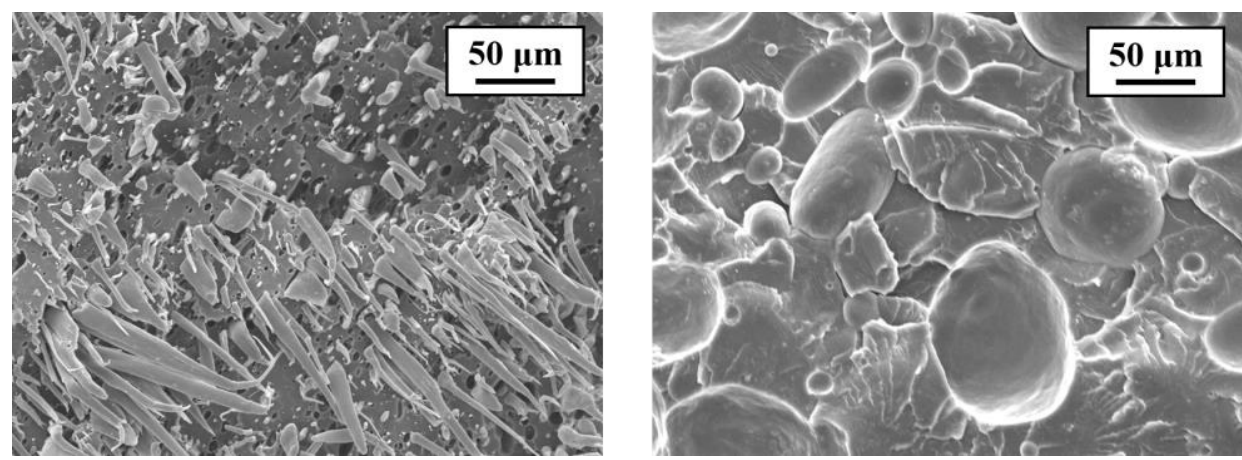

Figure 1.4 Scanning electron microscopy images representing the desired fibrillated LCP morphology for mechanical reinforcement (left) and the undesired nodular LCP morphology (right). Images taken are taken from cryogenically fractured composites consisting of $30 w t \%$ LCP in PLA.

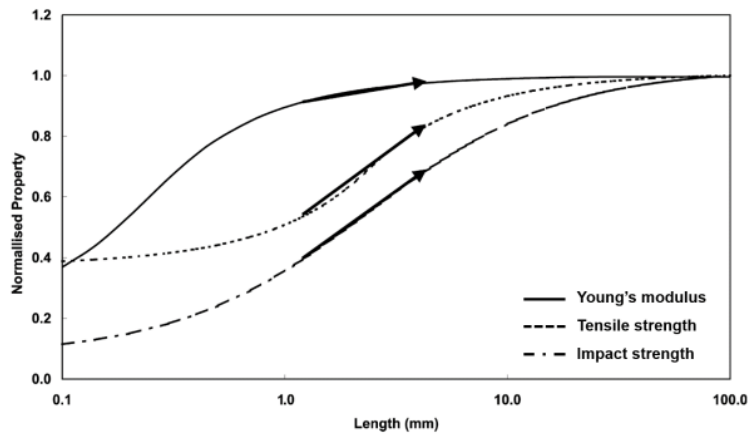

Figure 1.5 Young's modulus, Tensile strength, and Impact strength of a fiber-reinforced composite as a function of the fiber length. Adapted from Thomason ${ }^{27}$.

The performance of a composite depends not only on the nature of the different phases. It is affected by various factors such as the matrix-filler interface, the filler content, and the morphology of the filler. In fiber-reinforced composites, the fiber content, in combination with the orientation, length and diameter of the fibers largely determine the mechanical performance of the material ${ }^{9,27}$. For example, the Young's modulus can be increased by the incorporation of relatively short fibers, whereas longer fibers are required to increase the tensile strength and impact performance (Figure 1.5), as larger stresses need to be transferred from the matrix to the fiber in the latter cases. This is no different in LCP reinforced thermoplastic composites, where stretched LCP fibrils provide reinforcement ${ }^{23}$. However, the fibrillar morphology is not the equilibrium 
state of a molten blend, as the surface area of fibrils is larger than that of the energetically preferred spherical droplets. This means that the fibrillar morphology must be produced and frozen in during processing of the molten blend to prevent rapid relaxation into a spherical or nodular morphology (Figure 1.6, bottom).
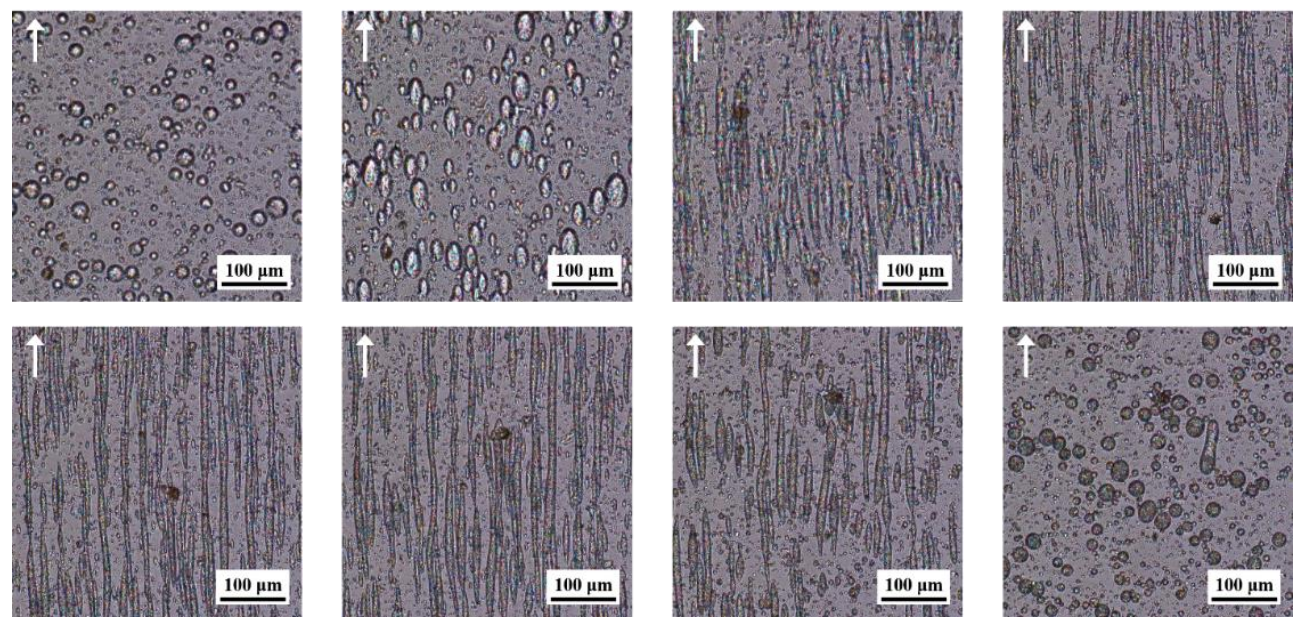

Figure 1.6 Development of the LCP morphology in a shear flow field tracked via optical microscopy. Images show a blend consisting of $10 \mathrm{wt} \%$ LCP in PLLA at $200{ }^{\circ} \mathrm{C}$. The white arrows mark the flow direction. Stretching of spherical LCP droplets upon the starting of flow (top). Relaxation of the stretched LCP fibrils upon the cessation of flow (bottom).

The formation of the blend morphology during processing is governed by three processes $^{28-30}$ : the deformation of droplets, the breakup of droplets, and the coalescence of droplets. In a deforming molten blend, the matrix flowing around a droplet imposes hydrodynamic forces on it, whereas the surface tension counteracts these forces. When the hydrodynamic forces on the droplet are larger than the surface forces, the droplet deforms (Figure 1.6, top images). With the uniaxial deformation of the droplet, the diameter decreases while its length increases. Concomitantly, the hydrodynamic forces acting on the droplet decrease, whereas the surface forces increase due to the increasing surface area. Eventually, this results in a situation where the stretched droplet is no longer stable and breaks up, creating smaller droplets. Upon contact, droplets may coalesce, forming a larger droplet. The interplay between these processes, during deformation, determines the resultant morphology. Breakup and coalescence have opposing effects on the average droplet size, and when given sufficient time in a constant flow field they will cancel out one another resulting in a dynamic equilibrium. These processes are dependent on many parameters, such as the viscoelastic behavior 
of the dispersed phase, the viscoelastic behavior of the matrix phase, the composition of the blend, and the applied processing conditions (eg. flow profile, cooling) ${ }^{31-35}$.

\subsection{Sustainability and reprocessing / recycling}

In the Cambridge dictionary, the word 'sustainability' is defined as 'the quality of causing little or no damage to the environment and therefore able to continue for a long time'. By that definition, the way modern-day society exploits our planet is not sustainable. We are highly dependent on fossil resources for our energy and materials, and we produce large amounts of waste that end up in the environment ${ }^{36,37}$. As a species, humanity is making an effort to become more sustainable, eg. by initiating a transition to more sustainable sources of energy such as wind, solar, or nuclear, and by attempting to clean up the waste we have produced. Nevertheless, much remains to be done.

Plastic materials, and plastic based composites, are currently not sustainable. They are largely produced from fossil feedstock, require a lot of energy and resources for their production and transport, and when plastic products end up in the environment, they are harmful to ecosystems 38,39 . However, plastics are too versatile, too useful, and simply too important to the daily operation of our society that their use can be avoided. Therefore, we need to use them smarter, in a more efficient and circular fashion. The sustainability problem of plastic materials is an extremely complex issue that lacks a single solution, but instead requires a combination of many solutions. However, it can be expected that both plastics from renewable, bio-based resources and efficient recycling of plastic materials in general are part of the solutions.

Currently, there are several distinct end-of-life scenarios available for plastic materials. The unfavorable scenarios, such as the plastic waste ending in the environment or its accumulation in landfill, need to be replaced by more feasible solutions, such as recycling ${ }^{38-42}$. Plastic materials can be recycled either via a mechanical route, a chemical route, or via energy recovery. The most efficient recycling route is material specific, and depends on the degree of degradation and contamination of the plastic ${ }^{43,44}$. The recycling of reinforced polymer composites comes with an additional challenge: the properties are strongly dependent on the reinforcing phase. During the mechanical recycling process, the reinforcing phase, often brittle glass- or carbon fibers, is damaged and broken up, causing a decrease in mechanical properties ${ }^{45-47}$. Solvolysis processes can be beneficial in the case of fibers that are resource intensive to produce (eg. carbon fiber), as the reinforcing fibers can be effectively reclaimed. However, these processes themselves are quite resource intensive, demand large quantities of harmful solvents, and lead to loss of the matrix ${ }^{48,49}$. 
Thermoplastic reinforced composites are highly suitable candidates for mechanical recycling without loss of performance ${ }^{24,26}$. The mechanical properties depend on the morphology that is formed during melt processing. If the viscoelastic behavior of the dispersed phase and matrix remains similar even after several reprocessing cycles, reprocessing of thermoplastic composites should be feasible using standard processing techniques such as extrusion.

Poly(lactide) (PLA) is one of the front-runners of bio-based polymers, and is produced on a commercial scale ${ }^{50}$. Due to good biocompatibility and non-toxic degradation products, it is a staple material in biomedical applications. Its glass transition temperature is in the proximity of $60^{\circ} \mathrm{C}$ and the material is generally stiff (Young's modulus 3-4 GPa), but brittle. The repeat monomer unit contains a stereocenter, and both D-lactic acid and L-lactic acid can be produced and used in the production process of PLA. The enantiomeric purity of PLA can be used to tune its properties: PLA with a high enantiomeric purity is a semi-crystalline material (although the crystallization typically proceeds slowly), whereas PLA with a low optical purity is amorphous. PLA is often labelled as a biodegradable polymer; however, this is only partially correct. The biodegradability of PLA is highly dependent on the environment to which it is exposed (eg. physiological conditions, soil, composting conditions), and greatly affected by the material parameters of the specific PLA (eg. molar mass, crystallinity) ${ }^{51}$.

That a polymer is bio-based does not necessarily mean it is sustainable, and therefore a closer look at the production and end-of-life scenarios of PLA is warranted. The commercial production of PLA starts with the production of biomass, typically corn or sugarcane, which is enzymatically hydrolyzed to dextrose and subsequently fermented in a biochemical process to produce lactic acid. This is used to produce a low molecular weight PLA via polycondensation and the obtained polymer is then depolymerized to form lactide (cyclic lactic acid dimer). Finally, the lactide is polymerized to obtain high molecular weight PLA by ring opening polymerization (ROP) ${ }^{50}$. The production of a kilogram of PLA has a considerably lower energy consumption and a smaller contribution to global warming compared to the production of the same amount of polyolefins (eg. poly(propylene) and poly(ethylene) $)^{52}$.

End-of-life scenarios of PLA products include compositing, thermomechanical recycling and chemical recycling. In composting the PLA is subjected to optimal conditions for biodegradation (eg. elevated temperatures and high humidity), and the polymer is metabolized by microorganisms. The effectiveness of this process is once again linked to the material parameters. Composting prevents that PLA waste products end up in the environment, and produces compost that can be used to produce new 
biomass, however, it is not the preferred end-of-life scenario for PLA. In thermomechanical recycling, the PLA waste is ground to pellets and subsequently melt processed to obtain a new product. This is a more feasible end-of-life scenario, as a new PLA product is obtained. However, the limited thermal stability in the melt can lead to gradual decrease in performance ${ }^{33,54}$. A very interesting aspect of PLA as a sustainable material is the efficiency with which it can be chemically recycled: it is considerably less resource intensive to produce PLA via chemical recycling than from biomass ${ }^{55}$. To recall, the chemical recycling of PLA simply requires depolymerization of the PLA to lactide, which can then be used to produce new PLA via ring opening polymerization. Compared to the production from biomass, chemical recycling does not involve additional steps beyond the collection and separation of the waste plastic, and does not require the initial, resource intensive steps that are necessary to obtain lactic acid. Based on the relatively environmental friendly production, and the availability of a highly feasible end-of-life scenario, it can be concluded that PLA has great potential as a sustainable plastic material (Figure 1.7).

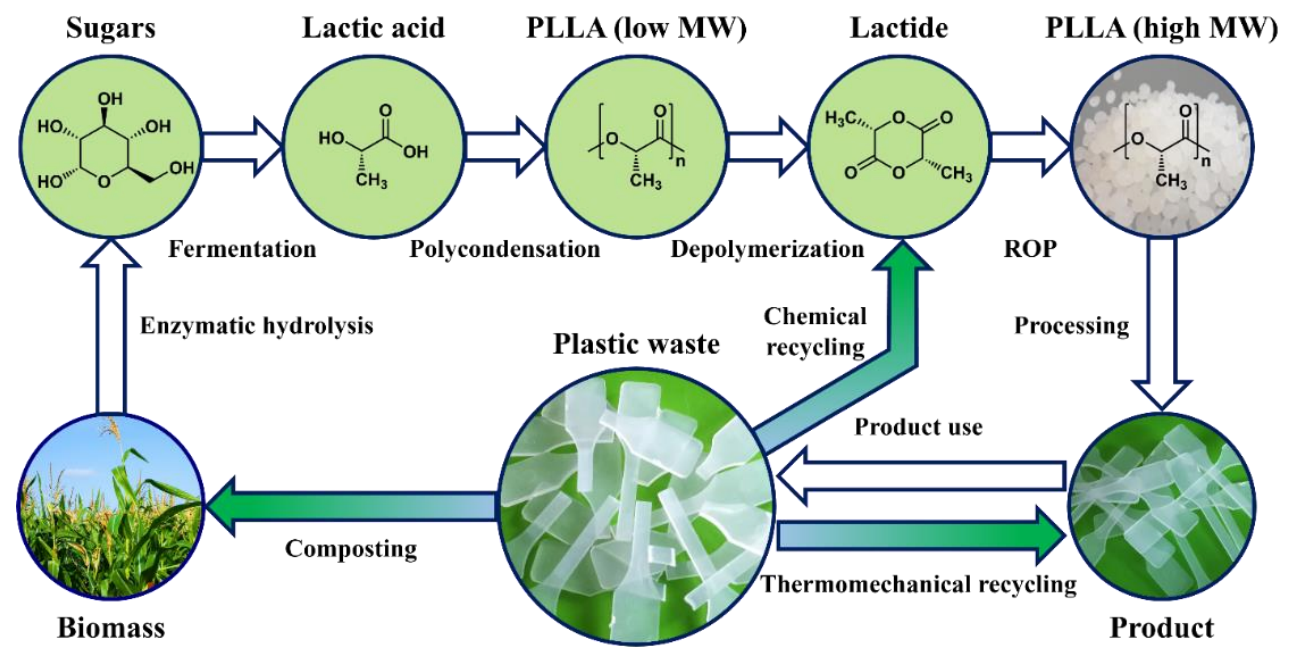

Figure 1.7 Overview of PLLA production and end-of-life scenarios for used PLLA products. 


\subsection{Aim and outline of this thesis.}

Thermotropic liquid crystalline polymers and their composites with thermoplastic matrices have been studied intensively in the past. In literature, effective reinforcement via a fibrillar LCP morphology has been demonstrated and reprocessability has been claimed as one of the advantages of thermoplastic composites. These materials have been lauded for their potential, but hardly any progress has been reported in the past twenty-fife years. The reason for this lies in the complexity that arises from the flow response of thermotropic LCPs and the different processes involved in the formation of a fibrillar morphology during processing. To explore the potential and eventually find commercial applications of these materials, a deeper understanding of the structure formation during (re)processing is required. The aim of this thesis is to study the flow behavior of LCPs and the processes that define the LCP morphology, and to use this understanding to develop a suitable system for the production of reprocessable LCP reinforced thermoplastic composites. PLA, being both bio-based and sustainable, is used as the matrix phase.

- A high level of interchain orientation is the cause for the exceptional properties of liquid crystalline polymers, either as a pure material or as a reinforcing phase in composites. In Chapter 2, the processes underlying the formation, and more importantly, the relaxation of the interchain orientation of liquid crystalline polymers are evaluated.

- The LCP morphology is essential for the performance of LCP-PLA composites. In Chapter 3, the influence of the used processing techniques and the effect of the thermal- and viscoelastic behavior of the LCP on the morphology and performance of thermoplastic composites is investigated.

- Reprocessability of LCP reinforced composites has often been used to exemplify the potential of these materials, however detailed studies proving recyclability are lacking. The reprocessability of LCP-PLA composites via a thermomechanical route and the influence of the molecular weight of the PLA matrix are investigated in Chapter 4.

- The thermal behavior of the LCP and the viscosity ratio of the two polymer in the blend were identified as important parameters regarding the performance of LCP reinforced thermoplastic composites. Therefore, a versatile system, based on LCPs with a low processing temperature and tunable viscosity, has considerable potential for commercial application. Chapter 5 elaborates on the synthesis of a liquid crystalline prepolymer, the controllable increase in molecular weight via reactive extrusion, and the characterization of the properties of the obtained LCPs with 
different molar masses. The relaxation of the orientation of the LCPs upon heating has been evaluated.

- In Chapter 6 the previously described LCPs with different molar masses and concomitantly different viscoelastic behavior are used to produce LCP reinforced thermoplastic composites with a PLA matrix via injection molding. Additionally, the influence of the matrix phase on the relaxation of the LCPs is studied.

- Chapter 7 addresses the potential of the materials and concepts described in this thesis. This includes industrial implementation, valorization opportunities, and concepts to further improve the performance or allow different types of applications. 


\subsection{References}

(1) Han, H.; Bhowmik, P. K. Wholly Aromatic Liquid-Crystalline Polyesters. Prog. Polym. Sci. 1997, 22 (7), 1431-1502. https://doi.org/10.1016/S0079-6700(96)00028-7.

(2) Chung, T.-S. The Recent Developments of Thermotropic Liquid Crystalline Polymers. Polym. Eng. Sci. 1986, 26 (13), 901-919. https://doi.org/10.1002/pen.760261302.

(3) Van Heeswijk, E. P. A.; Kragt, A. J. J.; Grossiord, N.; Schenning, A. P. H. J. Environmentally Responsive Photonic Polymers. Chem. Commun. 2019, 55 (20), 28802891. https://doi.org/10.1039/c8cc09672d.

(4) Timmermans, G. H.; Saes, B. W. H.; Debije, M. G. Dual-Responsive "Smart" Window and Visually Attractive Coating Based on a Diarylethene Photochromic Dye. Appl. Opt. 2019, 58 (36), 9823-9828. https://doi.org/https://doi.org/10.1364/AO.58.009823.

(5) Acierno, D.; Mantia, F. P. La; Polizzotti, G.; Ciferri, A.; Valenti, B. Ultrahigh Modulus Liquid Crystalline Polyesters. Macromolecules 1982, 15 (6), 1455-1460. https://doi.org/10.1021/ma00234a001.

(6) Picken, S. J.; Sikkema, D. J.; Boerstoel, H.; Dingemans, T. J.; van der Zwaag, S. Liquid Crystal Main-Chain Polymers for High-Performance Fibre Applications. Liq. Cryst. 2011, 38 (11-12), 1591-1605. https://doi.org/10.1080/02678292.2011.624367.

(7) Vita, F.; Adamo, F. C.; Pisani, M.; Heist, L. M.; Li, M.; Hegde, M.; Dingemans, T. J.; Samulski, E. T.; Francescangeli, O. Liquid Crystal Thermosets. A New Class of HighPerformance Materials. Liq. Cryst. 2019, 1-11. https://doi.org/10.1080/02678292.2019.1641233.

(8) Iqbal, M.; Picken, S. J.; Dingemans, T. J. Synthesis and Properties of Aligned AllAromatic Liquid Crystal Networks. High Perform. Polym. 2014, 26 (4), 381-391. https://doi.org/10.1177/0954008313516986.

(9) Tjong, S. C. Structure, Morphology, Mechanical and Thermal Characteristics of the in Situ Composites Based on Liquid Crystalline Polymers and Thermoplastics. Mater. Sci. Eng. R Reports 2003, 41, 1-60. https://doi.org/10.1016/S0927-796X(03)00035-4.

(10) Burghardt, W. R.; Brown, E. F.; Auad, M. L.; Kornfield, J. A. Molecular Orientation of a Commercial Thermotropic Liquid Crystalline Polymer in Simple Shear and Complex Flow. Rheol. Acta 2005, 44 (5), 446-456. https://doi.org/10.1007/s00397-004-0424-1.

(11) Beekmans, F.; Gotsis, A. D.; Norder, B. Transient and Steady-State Rheological Behavior of the Thermotropic Liquid Crystalline Polymer Vectra B950. J. Rheol. (N. Y. N. Y). 1996, 40 (5), 947. https://doi.org/10.1122/1.550788.

(12) Cuculo, J. A.; Chen, G. - Y. Extrusion, Fiber Formation, and Characterization of Thermotropic Copolyesters. J. Polym. Sci. Part B Polym. Phys. 1988, 26 (1), 179-200. https://doi.org/10.1002/polb.1988.090260110.

(13) Nguyen, T. N.; Geiger, K.; Walther, T. Flow Behavior of LCP Melts and Its Influence on Morphology and Mechanical Properties of Injection Molded Parts. Polym. Eng. Sci. 2000, 40 (7), 1643-1654. https://doi.org/10.1002/pen.11296.

(14) Wang, Y. L.; Yue, C. Y.; Tam, K. C.; Hu, X. Relationship between Processing, Microstructure, and Mechanical Properties of Injection Molded Thermotropic LCP. J. Appl. Polym. Sci. 2003, 88 (7), 1713-1718. https://doi.org/10.1002/app.11846.

(15) Gantenbein, S.; Masania, K.; Woigk, W.; Sesseg, J. P. W.; Tervoort, T. A.; Studart, A. R. Three-Dimensional Printing of Hierarchical Liquid-Crystal-Polymer Structures. Nature 2018, 561 (7722), 226-230. https://doi.org/10.1038/s41586-018-0474-7.

(16) Padias, A. B.; Hall, H. K. Mechanism Studies of LCP Synthesis. Polymers (Basel). 2011, 3 (2), 833-845. https://doi.org/10.3390/polym3020833. 
(17) Williams, P. A.; Han, X.; Padias, A. B.; Hall, H. K. Kinetics of the Polymerization of 4Acetoxybenzoic Acid and 6-Acetoxy-2-Naphthoic Acid. Macromolecules 1996, 29 (6), 1874-1879. https://doi.org/10.1021/ma951291c.

(18) Lodha, A.; Ghadage, R. S.; Ponrathnam, S. Polycondensation Reaction Kinetics of Wholly Aromatic Polyesters. Polymer (Guildf). 1997, 38 (25), 6167-6174. https://doi.org/10.1016/S0032-3861(97)00183-3.

(19) Jackson, W. J. Liquid Crystal Polymers. 4. Liquid Crystalline Aromatic Polyesters. Br. Polym. J. 1980, 12, 154-163. https://doi.org/10.1002/pi.4980120405.

(20) Economy, J. Aromatic Polyesters of P-Hydroxybenoic Acid. Mol. Cryst. Liq. Cryst. 1989, 169, 1-22. https://doi.org/10.1080/00268948908062731.

(21) Demus, D.; Goodby, J.; Gray, G. W.; Spiess, H. W.; Vill, V. Handbook of Liquid Crystals; Wiley-VCH, 1998. https://doi.org/10.1002/9783527620593.

(22) Collyer, A. A. Thermotropic Liquid Crystal Polymers for Engineering Applications Applications. Mater. Sci. Technol. 1989, 5, 309-322. https://doi.org/10.1179/mst.1989.5.4.309.

(23) Kiss, G. In Situ Composites: Blends of Isotropic Polymers and Thermotropic Liquid Crystalline Polymers. Polym. Eng. Sci. 1987, 27 (6), 410-423. https://doi.org/10.1002/pen.760270606.

(24) Bassett, B. R.; Yee, A. F. A Method of Forming Composite Structures Using in Situ Formed Liquid Crystal Polymer Fibers in a Thermoplastic Matrix. Polym. Compos. 1990, 11 (1), 10-18. https://doi.org/10.1002/pc.750110103.

(25) Bakis, C. E.; Bank, L. C.; Brown, V. L.; Cosenza, E.; Davalos, J. F.; Lesko, J. J.; Machida, A.; Rizkalla, S. H.; Triantafillou, T. C. Fiber-Reinforced Polymer Composites for Construction - State-of-the-Art Review. J. Compos. Constr. 2002, 6 (2), 73-87. https://doi.org/10.1061/(ASCE)1090-0268(2002)6:2(73).

(26) Wang, H.; Lee, K. W.; Chung, T. S.; Jaffe, M. Rheology, Morphology and Properties of LCP/Nylon 66 Composite Fibers. Polym. Compos. 2000, 21 (1), 114-123. https://doi.org/10.1002/pc.10170.

(27) Thomason, J. L. The Influence of Fibre Length and Concentration on the Properties of Glass Fibre Reinforced Polypropylene: 5. Injection Moulded Long and Short Fibre PP.

Compos. Part A Appl. Sci. Manuf. 2002, 33 (12), 1641-1652. https://doi.org/10.1016/S1359-835X(02)00179-3.

(28) Song, C. H.; Isayev, A. I. LCP Droplet Deformation in Fiber Spinning of SelfReinforced Composites. Polymer (Guildf). 2001, 42 (6), 2611-2619. https://doi.org/10.1016/S0032-3861(00)00555-3.

(29) Kamal, M. R.; Utracki, L. A.; Mirzadeh, A. Rheology of Polymer Alloys and Blends. In Polymer Blends Handbook, Volume 2; Utracki, L. A., Wilkie, C., Eds.; Spring Netherlands: Dordrecht, 2014; pp 726-853. https://doi.org/10.1007/978-94-007-6064-6.

(30) Utracki, L. A.; Shi, Z. H. Development of Polymer Blend Morphology during Compounding in a Twin-Screw Extruder. Part I: Droplet Dispersion and Coalescence. (A Review). Polym. Eng. Sci. 1992, 32 (24), 1824-1833. https://doi.org/10.1002/pen.760322405.

(31) Heino, M. T.; Hietaoja, P. T.; Vainio, T. P.; Seppälä, J. V. Effect of Viscosity Ratio and Processing Conditions on the Morphology of Blends of Liquid Crystalline Polymer and Polypropylene. J. Appl. Polym. Sci. 1994, $51 \quad$ (2), 259-270. https://doi.org/10.1002/app.1994.070510208.

(32) Tomotika, S. Breaking up of a Drop of Viscous Liquid Immersid in Another Viscous Fluid Which Is Extending at a Uniform Rate. Proc. R. Soc. 1936, 153, 302-318. https://doi.org/10.1098/rspa.1936.0003. 
(33) de Bruijn, R. A. Deformation and Breakup of Drops in Simple Shear Flows, PhD Thesis, Eindhoven University of Technology, 1989. https://doi.org/10.6100/IR318702.

(34) Delaby, I.; Ernst, B.; Germain, Y.; Muller, R. Droplet Deformation in Polymer Blends during Uniaxial Elongational Flow: Influence of Viscosity Ratio for Large Capillary Numbers. J. Rheol. (N. Y. N. Y). 1994, 38 (6), 1705-1720. https://doi.org/10.1122/1.550568.

(35) Bordereau, V.; Shi, Z. H.; Utracki, L. A.; Sammut, P.; Carrega, M. Development of Polymer Blend Morphology During Compounding in a Twin-Screw Extruder. Part III: Experimental Procedure and Preliminary Results. Polym. Eng. Sci. 1992, 32 (24), 18461856. https://doi.org/10.1002/pen.760322407.

(36) Janssens-Maenhout, G.; Crippa, M.; Guizzardi, D.; Muntean, M.; Schaaf, E.; Dentener, F.; Bergamaschi, P.; Pagliari, V.; Olivier, J. G. J.; Peters, J. A. H. W.; et al. Fossil CO2 \& GHG Emissions of All World Countries; 2017. https://doi.org/10.2760/709792.

(37) Hopewell, J.; Dvorak, R.; Kosior, E. Plastics Recycling: Challenges and Opportunities. Philos. Trans. R. Soc. B Biol. Sci. 2009, 364 (1526), 2115-2126. https://doi.org/10.1098/rstb.2008.0311.

(38) Perugini, F.; Mastellone, M. L.; Arena, U. A Life Cycle Assessment of Mechanical and Feedstock Recycling Options for Management of Plastic Packaging Wastes. Environ. Prog. 2005, 24 (2), 137-154. https://doi.org/10.1002/ep.10078.

Al-Salem, S. M.; Lettieri, P.; Baeyens, J. Recycling and Recovery Routes of Plastic Solid Waste (PSW): A Review. Waste Manag. 2009, 29 (10), 2625-2643. https://doi.org/10.1016/j.wasman.2009.06.004.

Asmatulu, E.; Twomey, J.; Overcash, M. Recycling of Fiber-Reinforced Composites and Direct Structural Composite Recycling Concept. J. Compos. Mater. 2014, 48 (5), 593-608. https://doi.org/10.1177/0021998313476325.

Shuaib, N. A.; Mativenga, P. T. Energy Demand in Mechanical Recycling of Glass Fibre Reinforced Thermoset Plastic Composites. J. Clean. Prod. 2016, 120, 198-206. https://doi.org/10.1016/j.jclepro.2016.01.070.

Rahimi, A. R.; Garciá, J. M. Chemical Recycling of Waste Plastics for New Materials Production. Nat. Rev. Chem. 2017, 1, 1-11. https://doi.org/10.1038/s41570-017-0046.

Luijsterburg, B. J.; Jobse, P. S.; Spoelstra, A. B.; Goossens, J. G. P. Solid-State Drawing of Post-Consumer Isotactic Poly(Propylene): Effect of Melt Filtration and Carbon Black on Structural and Mechanical Properties. Waste Manag. 2016, 54, 53-61. https://doi.org/10.1016/j.wasman.2016.04.029.

Choudhury, A.; Mukherjee, M.; Adhikari, B. Thermal Stability and Degradation of the Post-Use Reclaim Milk Pouches during Multiple Extrusion Cycles. Thermochim. Acta 2005, 430 (1-2), 87-94. https://doi.org/10.1016/j.tca.2004.12.025.

Eriksson, P. A.; Albertsson, A. C.; Boydell, P.; Prautzsch, G.; Månson, J. A. E. Prediction of Mechanical Properties of Recycled Fiberglass Reinforced Polyamide 66. Polym. Compos. 1996, 17 (6), 830-839. https://doi.org/10.1002/pc.10676.

Davies, P.; Le Duigou, A.; Pillin, I.; Baley, C.; Bourmaud, A. Effect of Recycling on Mechanical Behaviour of Biocompostable Flax/Poly(l-Lactide) Composites. Compos. Part A Appl. Sci. Manuf. 2008, 39 (9), 1471-1478. https://doi.org/10.1016/j.compositesa.2008.05.008.

Kuram, E.; Tasci, E.; Altan, A. I.; Medar, M. M.; Yilmaz, F.; Ozcelik, B. Investigating the Effects of Recycling Number and Injection Parameters on the Mechanical Properties of Glass-Fibre Reinforced Nylon 6 Using Taguchi Method. Mater. Des. 2013, 49, 139-150. https://doi.org/10.1016/j.matdes.2013.02.027. 
(48) Goto, M.; Sasaki, M.; Tokuno, S.; Shibata, K.; Iwaya, T. Recycling of Fiber Reinforced Plastics Using Depolymerization by Solvothermal Reaction with Catalyst. J. Mater. Sci. 2007, 43 (7), 2452-2456. https://doi.org/10.1007/s10853-007-2017-8.

(49) Morin, C.; Loppinet-Serani, A.; Cansell, F.; Aymonier, C. Near- and Supercritical Solvolysis of Carbon Fibre Reinforced Polymers (CFRPs) for Recycling Carbon Fibers as a Valuable Resource: State of the Art. J. Supercrit. Fluids 2012, 66, 232-240. https://doi.org/10.1016/j.supflu.2012.02.001.

(50) Auras, R. Poly(Lactic Acid) Synthesis, Structures, Properties, Processing, and Application; Auras, R., Lim, L., Selke, S., Tsuji, H., Eds.; John Wiley \& Sons, Inc.: Hoboken, 2010. https://doi.org/10.1017/CBO9781107415324.004.

(51) Kolstad, J. J.; Vink, E. T. H.; De Wilde, B.; Debeer, L. Assessment of Anaerobic Degradation of Ingeo ${ }^{\mathrm{TM}}$ Polylactides under Accelerated Landfill Conditions. Polym. Degrad. Stab. 2012, 97 (7), 1131-1141. https://doi.org/10.1016/j.polymdegradstab.2012.04.003.

(52) Vink, E. T. H.; Davies, S. Life Cycle Inventory and Impact Assessment Data for 2014 Ingeo ${ }^{\circledR}$ Polylactide Production. Ind. Biotechnol. 2015, 11 (3), 167-180. https://doi.org/10.1089/ind.2015.0003.

(53) Pillin, I.; Montrelay, N.; Bourmaud, A.; Grohens, Y. Effect of Thermo-Mechanical Cycles on the Physico-Chemical Properties of Poly(Lactic Acid). Polym. Degrad. Stab. 2008, 93 (2), 321-328. https:// doi.org/10.1016/j.polymdegradstab.2007.12.005.

(54) Badia, J. D.; Strömberg, E.; Karlsson, S.; Ribes-Greus, A. Material Valorisation of Amorphous Polylactide. Influence of Thermo-Mechanical Degradation on the Morphology, Segmental Dynamics, Thermal and Mechanical Performance. Polym. $\begin{array}{llll}\text { Degrad. Stab. } & \text { 2012, } & 97 & \text { (4), }\end{array}$ https://doi.org/10.1016/j.polymdegradstab.2011.12.019.

(55) Piemonte, V.; Sabatini, S.; Gironi, F. Chemical Recycling of PLA: A Great Opportunity Towards the Sustainable Development? J. Polym. Environ. 2013, 21 (3), 640-647. https://doi.org/10.1007/s10924-013-0608-9. 


\title{
Chapter 2 - Effect of shear rate on the orientation and relaxation of a vanillic acid based liquid crystalline polymer.
}

\begin{abstract}
Thermotropic liquid crystalline polymers are a class of materials with excellent properties for high performance applications. These properties come in large part from a certain state of interchain orientation on the macroscopic level, which is strongly influenced by deformation in the molten state. The transient response to shear and the relaxation upon cessation of flow are therefore important aspects in this class of materials. To get a better understanding of the underlying processes and identify the dominant parameters we analyzed the flow response of a novel bio-based LCP and analyzed the structure at different length scales by in situ POM and WAXD. Upon inception of shear, the polydomain texture is initially stretched, at larger strain breakup processes become increasingly important, and eventually a steady state texture is obtained. The shear stress response showed good coherence between optical and rheoWAXD data. The evolution of the orientation parameter coincides with the evolution of the texture: the order parameter increases as the texture stretches, drops slightly in the breakup regime, and reaches a constant value in the plateau regime. The relaxation of the shear stress and the polydomain texture showed two distinct processes with different timescales: The first is fast contraction of stretched domain texture, the second a slow coalescence of the polydomain texture. The timescale of the orientation parameter's relaxation matched with that of the slow coalescence process. All processes were found to scale with shear rate in the tested regime. These observations can have far-reaching implications for the processing of LCPs as they indicate that a higher shear rate during processing can correspond to an increased relaxation rate of the orientation parameter and therefore a decrease in anisotropy and material properties after cooling.
\end{abstract}

\section{Published as:}

de Kort, G. W.; Leoné, N.; Stellamanns, E.; Auhl, D.; Wilsens, C. H. R. M.; Rastogi, S. Effect of Shear Rate on the Orientation and Relaxation of a Vanillic Acid Based Liquid Crystalline Polymer. Polymers. 2018, 10, 935 


\subsection{Introduction}

Thermotropic liquid crystalline polyesters (LCPs) are an excellent class of materials for high performance applications. The liquid crystalline behavior above the crystal melting temperature results in low thermal expansion coefficients, low viscosities and introduces the potential to reach high strength and modulus when processed correctly ${ }^{1-}$ ${ }^{6}$. In main-chain LCPs, these properties originate from the rigid chemical structure of the polymer backbone; the mesogenic moieties in the backbone provide a high aspect ratio to the polymer and limits the formation of entanglements 5 . Instead of the usual entangled isotropic melt, the LCPs can be described as a suspension of domains in which preferred directional order exists 7 . This behavior represents the key in achieving excellent mechanical properties upon unidirectional orientation; due to the molecular orientation inside the nematic domains, imposed stresses to the material can actively be carried by the polymer chains instead of the entanglement network ${ }^{6}$.

The well-known unidirectional properties of LCPs can only be obtained in the case coherent orientation on a macroscopic level is achieved. By far, the most efficient route to obtain macroscopic orientation in thermotropic LCPs is through the application of an elongational flow field, as is the case during the fiber spinning process. A thermotropic polyester whose processing behavior has been extensively studied is the copolymer of poly(ethylene terephthalate) modified with $60 \mathrm{~mol} \%$ of hydroxybenzoic acid. This polymer exhibits a broad melting range, characteristic for LCPs, and is processable at $260^{\circ} \mathrm{C}$. From literature two characteristic routes are reported to process this LCP: The first route is reported by Muramatsu and Krigbaum, who demonstrated that molecular orientation is already induced by the application of shear and contraction forces in the capillary of a capillary rheometer ${ }^{8-10}$. As this pre-oriented melt exited the capillary die, no extensive drawing was required to achieve high modulus fibers. These observations are in line with slit contraction experiments ${ }^{11}$. Processing via this route proved to yield better mechanical properties when performed at processing temperatures of $260^{\circ} \mathrm{C}$ and higher. In contrast to the first route, the second route relies only on heavy spin-drawing to induce molecular orientation, as has been reported by Acierno and coworkers ${ }^{6}$. Interestingly, for this processing route a decrease of the processing temperature to $225^{\circ} \mathrm{C}$ resulted in significant enhancement of the mechanical performance. These results make it clear that, when both shear and flow components before and after the die are to be taken into account, processing of LCPs becomes rather complex. For example, higher spinning temperatures yield better results in the case orientation is induced via shear, while the opposite relation holds in case the orientation is induced by elongation ${ }^{12,13}$. In addition, draw ratio, temperature, block formation 
(rearrangements along the backbone at high temperatures) and flow history can influence the orientation in the fiber, and therefore yield a direct response in fiber mechanical properties. The degree of orientation is not only an important factor regarding the mechanical response of thermotropic LCP fibers, but has proven to be critical in their composites with thermoplastics as well ${ }^{14-17}$.

For this particular reason, and to generate fundamental understanding on the rheological behavior of thermotropic LCPs, the response of LCPs to shear flow fields has been extensively studied over the last decades. In order to gain deeper understanding of the structural background of this complex rheological behavior, it is essential that multiple time- and length scales can be observed simultaneously. Examples of LCPs that have been studied include the Vectra copolymers, PET-HBA copolymers, and stilbene based LCPs ${ }^{11-13,18-23}$. A general feature in transient shear flow of these LCPs is that their viscosity and shear stress exhibit two peaks separated by a minimum before reaching a steady state value. These features are generally attributed to the evolution of the domain texture and the interchain orientation, though their exact relation remains a topic for discussion. This response appears strongly dependent on various parameters including, molecular composition of the LCP, experimental temperatures, flow- and thermal history ${ }^{11,19,24-30}$. Despite extensive research and discussions on the complex rheological behavior LCPs, a unifying theory capable of describing flow behavior of thermotropic LCPs is still absent. Additional challenges arise from the inaccessibility of the isotropic state for commercially relevant aromatic thermotropic LCPs ${ }^{11}$, whereas the presence of flexible aliphatic units in model systems with low transition temperatures might lead to different behavior ${ }^{24}$.

Recently, we have reported on the synthesis and processing of a series of thermotropic LCPs based on vanillic acid ${ }^{14,31,32}$. By choosing the right monomers, high-end polymer materials can be obtained, either fully or in large part, from bio-based sources. These materials can readily be synthesized at mild reaction temperatures $\left(\leq 260{ }^{\circ} \mathrm{C}\right)$ yielding molecular weights $\geq 10 \mathrm{~kg}$ mole $^{-1}$ which allow for fiber spinning. The low melting point of $126{ }^{\circ} \mathrm{C}$ allows for melt-processing in the nematic phase at $150{ }^{\circ} \mathrm{C}$. Melt-processing of these materials yielded highly oriented fibers with a tensile modulus of 10-12 GPa following the method of Acierno and coworkers through extensive spin-drawing ${ }^{32}$. Further investigation revealed that these vanillic acid based LCPs undergo rapid molecular relaxation during processing. Such rapid relaxation of molecular orientation might limit their application as LCPs are generally used as unidirectional reinforcing polymers, thereby requiring a high orientation parameter in the processing direction. To obtain more insight into the orientation and relaxation processes involved, during and after flow, in these vanillic acid based aromatic-aliphatic thermotropic polyesters, in this 
study we report the rheological response of these materials analyzed by in situ WAXD and polarization optical microscopy.

\subsection{Methods and Materials}

Suberic acid, 1,4-diacetoxybenzene, and 4-hydroxy-3-methoxybenzoic acid were purchased from Sigma-Aldrich. $p$-Acetoxybenzoic acid was purchased from TCI Europe.

\section{Acetylation of 4-hydroxy-3-methoxybenzoic acid.}

4-Hydroxy-3-methoxybenzoic acid (50 g, $304.5 \mathrm{mmol}$ ) was placed in a $250 \mathrm{~mL}$ roundbottom flask on a magnetic stirring plate. Dry acetic anhydride $(50 \mathrm{~mL}, 530 \mathrm{mmol})$ was added together with a catalytic amount of $\mathrm{H}_{2} \mathrm{SO}_{4}$. The mixture was heated to $80{ }^{\circ} \mathrm{C}$ and left to react for $6 \mathrm{~h}$. After cooling the mixture to $0{ }^{\circ} \mathrm{C}, 200 \mathrm{~mL}$ of water was added, and the obtained yellow crystals were filtered, washed with water, and dried in vacuo at $60{ }^{\circ} \mathrm{C}$ for $14 \mathrm{~h}$.

\section{Polymerization Procedure.}

The polymerization was performed in a $100 \mathrm{~mL}$ three-neck round-bottom flask fitted with a mechanical stirrer. The monomer mixture consisting of $p$-acetoxybenzoic acid (29.1 g, $162 \mathrm{mmol}$ ), suberic acid (28.2 g, $162 \mathrm{mmol})$, 1,4-diacetoxybenzene (31.4 g, 162 $\mathrm{mmol})$, and 4-acetoxy-3-methoxybenzoic acid (11.3 g, $54 \mathrm{mmol}$ ) was introduced together with $60 \mathrm{mg}$ of $\mathrm{Zn}(\mathrm{AcO})_{2}$ to the round-bottom flask. The monomers were dried overnight in vacuo at $40{ }^{\circ} \mathrm{C}$ prior to usage to eliminate moisture. Furthermore, after loading of the monomers the round-bottom flask was iteratively flushed with argon and reduced pressure for three times prior to the start of the reaction to minimize oxygen content. Next, a small argon flow was applied to the system and the temperature was increased stepwise to $200^{\circ} \mathrm{C}$. As soon as acetic acid started to be formed, the reaction temperature was gradually increased to $260^{\circ} \mathrm{C}$ after which the polymerization was allowed to proceed for $6 \mathrm{~h}$. Next, reduced pressure was applied to the system for $12 \mathrm{~h}$ to build up molecular weight. The final product was isolated from the hot reactor flask in the form of a polymer melt.

\section{Material Characterization.}

The molecular weight distribution was determined via size exclusion chromatography (SEC). The polymer was dissolved in 1,1,1,3,3,3-hexafluoroisopropanol (HFIP) containing $0.019 \%$ sodium trifluoroacetate. After dissolution the sample was filtered over a $0.2 \mu \mathrm{m}$ PTFE filter prior to injection. The system is equipped with a Waters 1515 Isocratic HPLC pump, a Waters 2414 refractive index detector $\left(40{ }^{\circ} \mathrm{C}\right)$, a Waters 2707 autosampler, and a PSS PFG guard column followed by 2 PFG-linear-XL ( 7 mm, $8 \times$ 
$300 \mathrm{~mm}$ ) columns in series at $40^{\circ} \mathrm{C}$. HFIP (Apollo ScientificLimited) with potassium trifluoroacetate $\left(3 \mathrm{~g} \mathrm{~L}^{-1}\right)$ was used as an eluent at a flow rate of $0.8 \mathrm{~mL} \mathrm{~min}{ }^{-1}$. The molecular weights were calculated against poly(methyl methacrylate) standards (Polymer Laboratories, $M_{\mathrm{p}}=580 \mathrm{Da}$ up to $M_{\mathrm{p}}=7.1 \times 10^{6} \mathrm{Da}$ ).

The glass transition temperature $\left(T_{\mathrm{g}}\right)$ and the peak temperature of the crystalline-tonematic transition $\left(T_{\mathrm{m}}\right)$ were determined by differential scanning calorimetry (DSC) using a TA Instruments Q2000 DSC. The heating and cooling-rates of the sample were $10{ }^{\circ} \mathrm{C} \mathrm{min}^{-1}$ and measurements were performed under a nitrogen rich atmosphere.

The evolution of the melt polydomain texture was evaluated under crossed polarizers using an Olympus BX53 Microscope (20x magnification) equipped with an Olympus DP26 camera. The microscope was operated in transmission. The samples were loaded in a Linkam CSS 450 shear cell that was preheated to $200{ }^{\circ} \mathrm{C}$ and subsequently squeezed until a gap of $50 \mu \mathrm{m}$ was reached. The sample was kept in the shear cell for 10 minutes prior to the measurement to prevent temperature fluctuations and to allow relaxation of the flow history. During the experiments, the samples were sheared until a strain of at least 200 strain units had been applied in order to ensure that the steady state texture was reached. Subsequently, the relaxation of the polydomain texture was evaluated over a 10-minute period following the cessation of shear. The applied shear rate was calculated based on the position of the aperture in the shear cell, the angular rotation rate of the rotating plate, and the sample thickness, under the assumption of a linear velocity profile and no slip conditions at the glass plates.

$\mathrm{X}$-ray scattering experiments were performed at the rheo-X end station of beamline P10, situated at the German electron synchrotron PETRA III of DESY Hamburg. Given the high brilliance of PETRA III in combination with the flux and wavelengths provided at P10 $(5-25 \mathrm{keV})$, the beamline is particularly well suited for time resolved Xray diffraction studies on soft matter and complex fluids. The Small-angle X-ray Scattering rheo SAXS setup of P10 was built around an inverted Haake MARS II rheometer, invented by B. Struth et al. and manufactured by Thermo Fisher in collaboration with DESY Hamburg ${ }^{33}$. In contrast to horizontal configurations, it utilizes a vertical Bragg reflex of the primary beam provided by a Silicon crystal (Figure 2.1). 


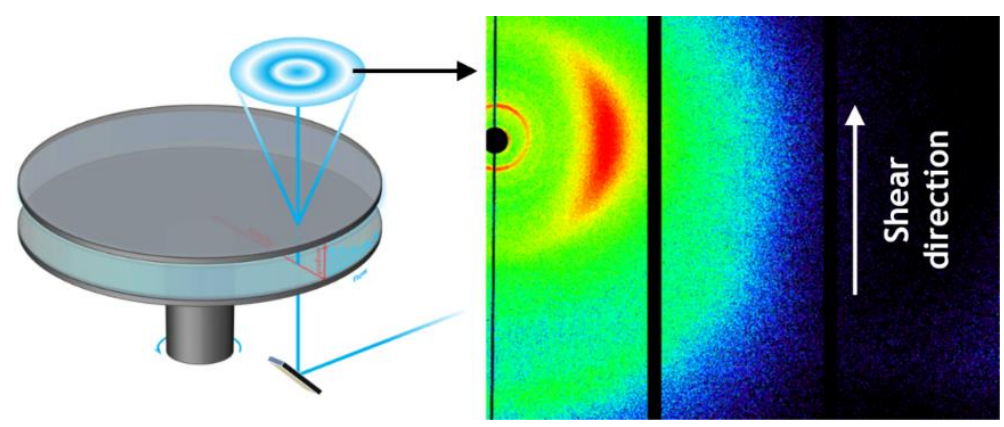

Figure 2.1 Schematic representation of the vertical beam path and the coordinate system of the plate-plate sensor at beamline P10 and the characteristic WAXD pattern from the LCP scattering signal observed during continuous shear at a rate of $10 \mathrm{~s}^{-1}$.

With the motor positioned below the sensor the beam can pass through the sample all the way up to the X-ray detector. The vertical beam provides a unique point of view on the structure of the sample, probing the electron density distribution parallel to the gradient of shear and perpendicular to the vorticity plane using a plate-plate geometry. The probed volume is defined by the size of the beam and the height of the gap as opposed to the length of the secant probed in horizontal configurations, where the signal is averaged over a range of shear rates and partially disturbed at the meniscus. The accessible q-range of the setup has been expanded from Small to Wide-angle X-ray Diffraction (WAXD) to also observe shear induced changes on the molecular scale, hence the term rheo-X. In combination with a newly developed high temperature probe environment it is now possible to conduct time resolved structural rheology on polymer melts on both the micro and the nano scale in situ. At a photon energy of $13.980 \mathrm{keV}$ we used an $\mathrm{Si}[555] \mathrm{X}$-ray reflex to probe the sample in a $\mathrm{D}=35 \mathrm{~mm}$ plate-plate geometry built from a high precision aluminum sensor, originally provided by Thermo Fisher and modified in-house to provide X-ray windows, altogether covered by a single $75 \mu \mathrm{m}$ Kapton sheet. The beam dimensions were set to 200 x $200 \mu \mathrm{m}^{2}$. A Pilatus $300 \mathrm{k}$ 2D X-ray detector was positioned at a distance of $115 \mathrm{~mm}$ away from the sample. The samples were loaded in the rheo-X set up that was preheated to the measurement temperature and subsequently squeezed to a thickness of $500 \mu \mathrm{m}$. Excess material was removed. The material was allowed to relax and equilibrate for 10 minutes before proceeding with the measurement.

The orientation parameter $(S),\left\langle\mathrm{P}_{2 \mathrm{n}}(\cos \varphi)\right\rangle_{\mathrm{d}}$, was calculated via the procedure described by Mitchell and Windle ${ }^{34}$. The azimuthal intensity $I(\varphi)$ at the maximum of the interchain diffraction peak $\left(2 \theta=21^{\circ}\right)$ was taken. The orientation parameter $<\mathrm{P}_{2 \mathrm{n}}(\cos$ $\varphi)>_{d}$ was then determined from an average of a Legendre polynomial, weighted against 
the obtained azimuthal intensity scan using equations (1) - (3). In this case, only the second order Legendre polynomial was taken into account, $\left\langle\mathrm{P}_{2 \mathrm{n}}(\cos \varphi)\right\rangle_{\mathrm{m}}=-0.5$.

$$
\begin{gathered}
\mathrm{S}=<\mathrm{P}_{2 n}(\cos \varphi)>_{d}=\frac{<\mathrm{P}_{2 n}(\cos \varphi)>}{\left.<\mathrm{P}_{2 n}(\cos \varphi)\right\rangle_{m}} \\
<\mathrm{P}_{2 n}(\cos \varphi) \geq \frac{\int_{0}^{\frac{\pi}{2}} I(\theta, \varphi) \mathrm{P}_{2 n}(\cos \varphi) \sin \varphi \delta \varphi}{\int_{0}^{\frac{\pi}{2}} I(\theta, \varphi) \sin \varphi \delta \varphi} \\
<P_{2 n}(\cos \varphi)>_{m}=\frac{(2 n) !}{(-1)^{n} 2^{2 n}(n !)^{2}}=-\frac{1}{2} \text { for the second term }
\end{gathered}
$$

The obtained orientation parameter reflects the contributions of the distribution of the director orientation throughout the bulk poly-domain sample and the contributions of the director on a molecular level ${ }^{22}$. In short, the orientation parameter reflects the degree of anisotropy of the scattering of polymer chains, while assuming that these chains are infinitely long rigid rods. The values of $\mathrm{S}$ vary from 0 , corresponding to a random chain orientation similar to the orientation of an isotropic liquid, to unity, corresponding to the perfect alignment of the polymer chains along the orientational axis.

\subsection{Results and Discussion}

\section{LCP synthesis and characterization}

The LCP investigated in this study is a copolymer of vanillic acid, suberic acid, hydroquinone, and $p$-hydroxy benzoic acid, which was synthesized according to the procedure described by Wilsens et al. ${ }^{31}$ (Figure 2.2a). The studied material has a weight average molecular weight of $22 \mathrm{~kg}^{-10 \mathrm{e}^{-1}}$ and a PDI of 4.7 determined by HFIP-GPC analysis (Figure 2.2b). The molecular weight distribution shows no shoulder on the high molecular weight side, indicating that the copolymer has a random nature and that block formation did not play a significant role ${ }^{31,35}$. The aromatic aliphatic LCP is thermotropic in nature as it shows a stable nematic phase above its crystal melting temperature. The melting and crystallization behavior of the LCP as observed from DSC analysis is depicted in Figure 2.2c. Degradation of the melt occurred above $300{ }^{\circ} \mathrm{C}$, but no isotropisation was observed prior to degradation. 


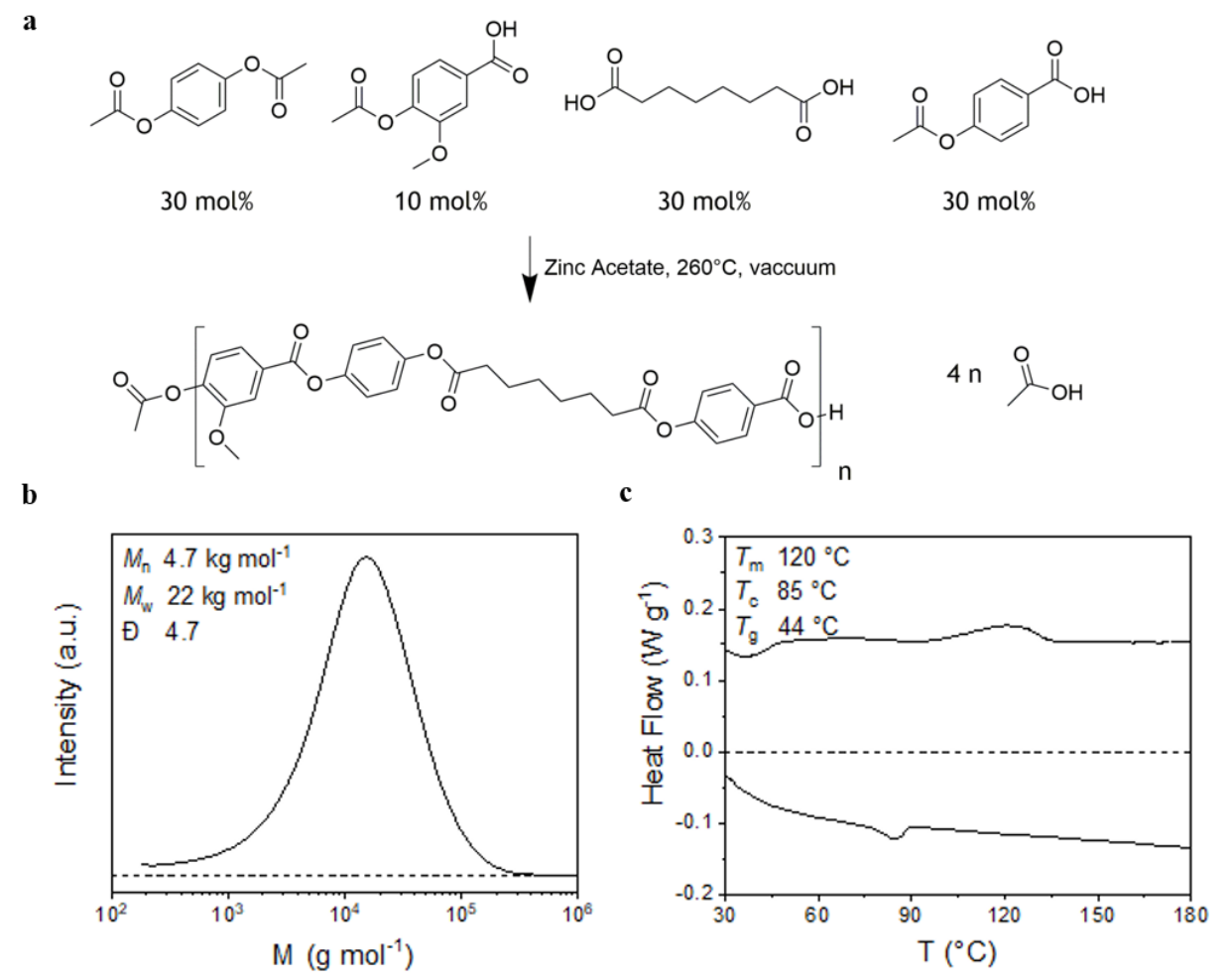

Figure 2.2 (a) Reaction scheme of the LCP synthesis, resulting in a random copolymer. (b) Molecular weight distribution of the LCP. (c) DSC thermogram (endo up) depicting the cooling and second heating of the LCP taken at a rate of $10^{\circ} \mathrm{C} \mathrm{min}^{-1}$.

\section{Analysis of texture development}

To recall, melt-processing of this LCP followed by extensive spin drawing with draw ratios of around 1000 yielded fibers with a high orientation parameter $(S \sim 0.85-0.9)^{32}$. We anticipate that the high spin-drawing velocities is desired to counteract rapid molecular relaxation of the LCP chains during processing. To obtain further insight on the orientation and relaxation process of the LCP, shear experiments were performed in a Linkam Shear-cell in the thermotropic melt, and the textural changes were monitored with polarization optical microscopy. It is well known that thermotropic LPCs exhibit a domain texture in which the chains are molecularly aligned with respect to the director, whereas the director varies for each domain. Upon deformation of the melt, the polydomain texture initially deforms and orients, contributing to the overall molecular alignment of the LCP chains ${ }^{13,36,37}$. At larger deformations breakup and coalescence processes become increasingly important, until the liquid crystalline melt reaches a state of equilibrium in terms of both domain texture and molecular 
orientation. The equilibrium polydomain textures of the LCP studied in this work were compared via polarized optical microscopy (POM, Figure 2.3) under different flow conditions.
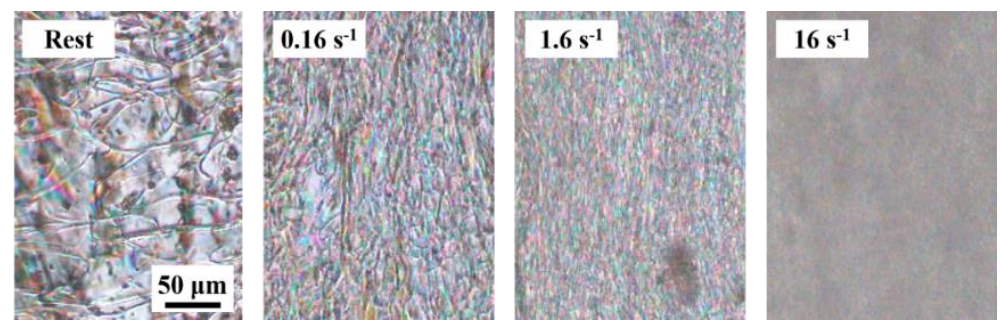

Figure 2.3 Equilibrium polydomain textures of the LCP under different shear rates at $200{ }^{\circ} \mathrm{C}$. The total strain was greater than 200 for each sheared sample. Flow direction is vertical.

Figure 2.3 depicts the birefringent domains that make up the polydomain texture of the LCP. The melt can be characterised as a nematic Schlieren texture, showing deformation dependent disclination lines ${ }^{3}$. The domain size is inversely proportional to the applied shear rate. For example, domain features in the order of $10 \mu \mathrm{m}, 1 \mu \mathrm{m}$, and $<1 \mu \mathrm{m}$, are found at shear rates of $0.16 \mathrm{~s}^{-1}, 1.6 \mathrm{~s}^{-1}$, and $16 \mathrm{~s}^{-1}$, respectively. Similar observations were made by Guo et al., during in situ measurements of light transmission of the thermotropic Vectra LCP V400P copolyester under shear flow ${ }^{12}$. These authors attributed the decrease in optical intensity with increasing shear rate to increased defect density and decreased domain size. In addition to domain breakup, the application of shear to the nematic polydomain texture also results in stretching of the domains in the direction of the shear field. Such domain deformation is clearly visible from Figure 2.3, in particular for the image depicting the optical morphology at the steady shear at 0.16 $\mathrm{s}^{-1}$.

The relaxation process after cessation of flow, depicted in Figure 2.4, for the lowest shear rate of $0.16 \mathrm{~s}^{-1}$ shows that the initially stretched polydomain texture relaxes back to an un-stretched state over the course of 100 seconds, followed by coalescence. Generally, both processes are relatively slow, and the domain size changes over only one order of magnitude during the full process. At a shear rate of $1.6 \mathrm{~s}^{-1}$ (depicted in Figure 2.5), the polydomain texture at cessation of flow is stretched and the domain size is considerably smaller. The same two relaxation processes are observed, although they occur at considerably shorter timescales; 10 seconds after the cessation of flow, the polydomain texture no longer appears stretched and from that point onwards the main process is coalescence of the polydomain texture. Unfortunately, direct observation of the relaxation of a stretched texture followed by coalescence is not possible from the optical images after the application of a shear rate of $16 \mathrm{~s}^{-1}$, due to the small domain size 

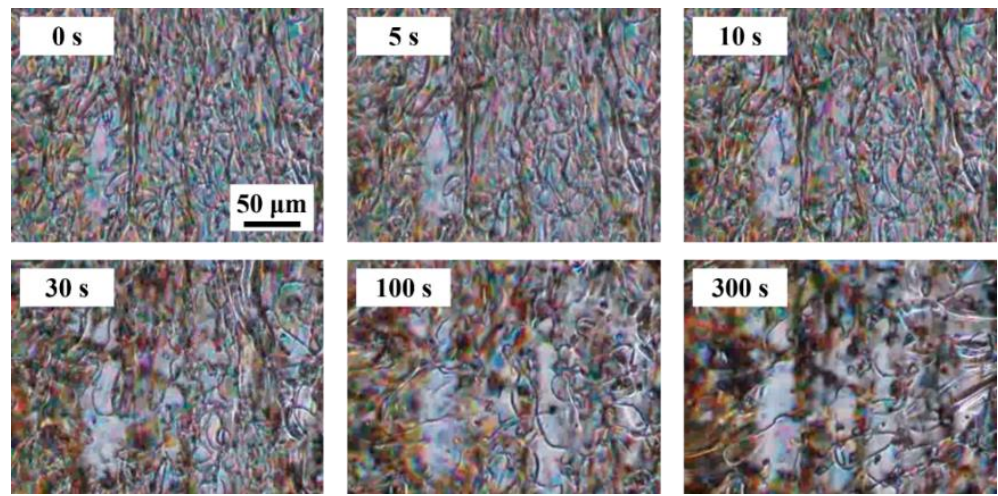

Figure 2.4 Relaxation from equilibrium texture of the LCP after the application of shear $\left(0.16 s^{-1}\right)$ at 200 ${ }^{\circ} \mathrm{C}$. Flow direction is vertical.
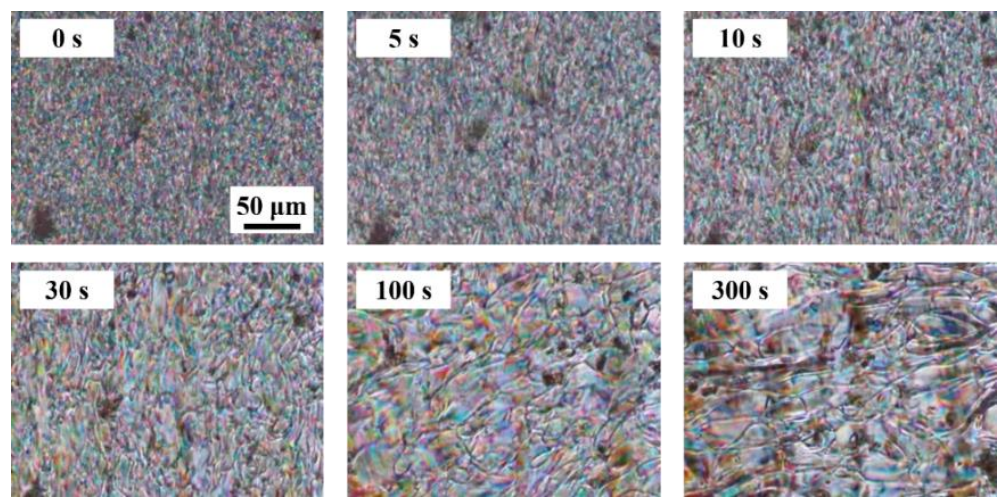

Figure 2.5 Relaxation from equilibrium texture of the LCP after the application of shear $\left(1.6 s^{-1}\right)$ at $200{ }^{\circ} \mathrm{C}$. Flow direction is vertical.
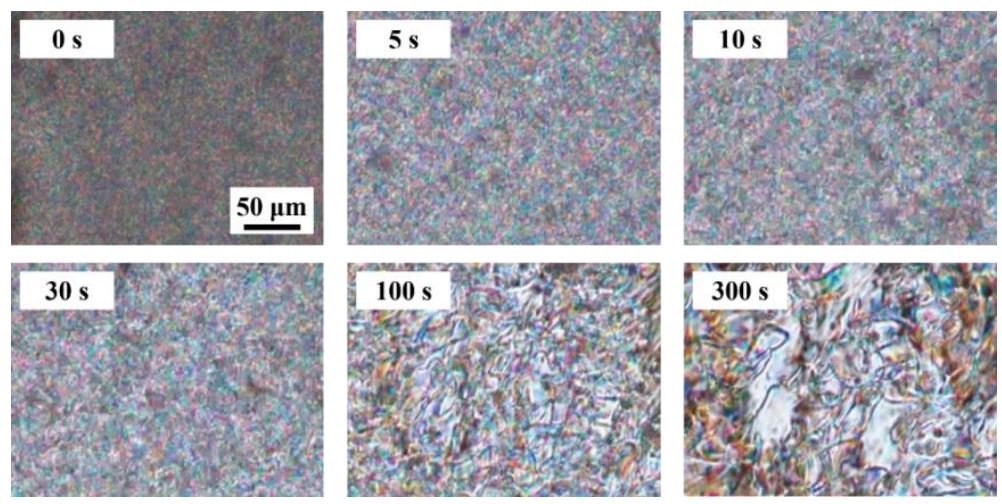

Figure 2.6 Relaxation from equilibrium texture of the LCP after the application of shear $\left(16 \mathrm{~s}^{-1}\right)$ at $200{ }^{\circ} \mathrm{C}$. Flow direction is vertical. 
and the high fluid velocity (Figure 2.6). That being said, considerable brightening occurs within 5 seconds after cessation of shear. After that, the polydomain texture continues to coalesce. Though not visible, it stands to reason to assume that the initial relaxation, dominated by the contraction of the stretched texture, would have occurred completely within the timeframe of 5 seconds. Over time, coalescence continues for all shear rates until a texture close the texture prior to shear is obtained after roughly 300 s.

The textural relaxation of the investigated LCP appears to be governed by two distinct processes; 1) The dissipation of elastic energy, via contraction, stored in the stretched polydomain texture which occurs within the first seconds after cessation of shear, and 2) reduction of the interdomain surface area through domain coalescence which proceeds within minutes. The timescale for both relaxation processes seems to speed up with an increase in the previously applied shear rate. Such behavior can be expected as the steady state textural morphology differs increasingly with increasing shear rate. In other words, the polydomain texture becomes finer and more stretched as the shear rate increases, and thus the amount of excess energy stored in the system, both in the form of interdomain surface area and a stretched texture, increases. Consequently, the more energy stored in the texture, the larger the driving force to relax back to a low energy mono-domain morphology, as is also observed for lyotropic systems ${ }^{38}$. It should be noted that the argument made based on the rheo-optical data is only qualitative, as accurate determination of domain sizes proved challenging due to the decreased quality of the images at high shear rates. Additionally, some flow perpendicular to the shear direction was observed upon cessation of flow, due to bulk flow inertia, which is not uncommon in relatively low viscous liquids at high shear rates.

\section{Rheo-WAXD}

Complementary to the rheo-optical experiments, rheology in combination with in situ Wide-angle X-ray Diffraction (Rheo-WAXD) analysis is used to correlate the viscoelastic behavior to the molecular orientation and relaxation of the LCP in a shear flow field. In general, flow curves of LCPs can exhibit three characteristic regimes ${ }^{20,28,29,39-42}$. In Regime I, at low shear rates, shear thinning occurs as a result of coherent motion of domains. Regime II is characterized by strong changes in the texture and some degree of molecular orientation in the flow direction, which generally occurs at intermediate shear rates. Regime III, also known as the second shear thinning regime, is generally found at even higher shear rates and is associated with the gradual disappearance of defects and the formation of a highly oriented monodomain. Firstly, a flow curve was constructed from the transient experiments by plotting the steady state viscosity of the LCP as a function of shear rate (Figure 2.7). As can be seen from Figure 
2.7, at shear rates between $0.1 \mathrm{~s}^{-1}$ and $3.0 \mathrm{~s}^{-1}$, the viscosity is constant indicating that the LCP resides in Regime II. This is in good agreement with in situ POM (Figure 2.3), where a strong dependence of domain texture on shear rate was found. At shear rates of $10 \mathrm{~s}^{-1}$ and higher, the onset of the second shear thinning regime is observed, marking the transition between Regime II and III.

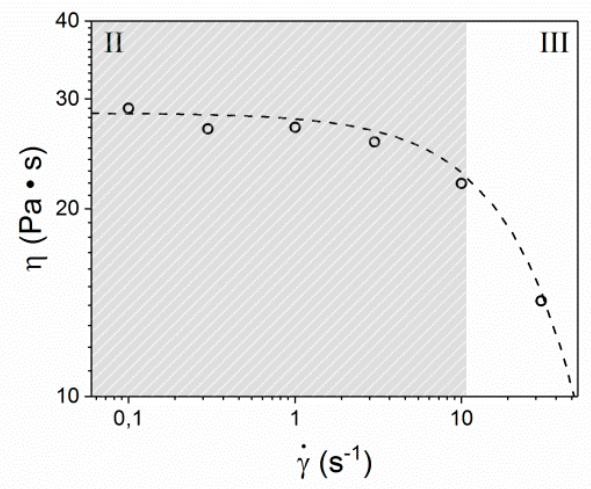

Figure 2.7 Steady state viscosity as a function of shear rate at $150{ }^{\circ} \mathrm{C}$. Regime II is marked in gray.

In addition to the evaluation of the steady state viscosity (Figure 2.7), the start-up of shear has been monitored during the application of varying shear rates. This transient behavior of the LCP is depicted in Figure 2.8 for shear rates of $0.1,1$, and $10 \mathrm{~s}^{-1}$. It is well known from literature, the transient behavior of polydomain thermotropic LCPs is not only complex, but also strongly dependent on sample history and microstructure $11,19,23-30,43$. It is generally accepted that the materials response is related to the evolution of the domain texture and the interchain orientation, but the exact relations remain under discussion. The most general response of thermotropic nematic melts to the inception of shear stress involves four regimes (A-D); Regime A shows a peak in the shear stress at low strain values, followed by a minimum in shear stress, defining regime $\mathrm{B}$. A second stress overshoot at large strain (regime $\mathrm{C}$ ) is often observed before a steady state is reached (regime D). Measurements by Guo et al. on an amorphous, fully aromatic thermotropic LCP indicate that the first stress overshoot (A) and the subsequent minimum (B) correspond to stretching and break down of the domain texture at $\gamma=0-20$, while the second overshoot $(C)$ is related to the evolution of molecular orientation at $\gamma=20-300{ }^{12,13}$. Burghardt $e t$ al. found that, for a different fully aromatic LCP, the main increase in the orientation parameter obtained from WAXD occurred at considerably lower strain $(\gamma=0-40)$, whereas the overall stress profile was similar ${ }^{11}$. This emphasizes the generic nature of the evolution of the shear stress, but the challenges in correlating that to the evolution of the interchain orientation. Independent of differences in their findings regarding the interchain 
orientation, both authors stress the importance of the samples history before the transient. In both studies qualitatively similar procedures were used, and the starting point was a polydomain structure as a result of a squeeze flow during sample loading that had been allowed to relax for some time. In situ rheo-WAXD provides a powerful tool that can aid to elucidate the complex transient behavior of LCPs and the correlated structural changes. Due to their correlation with the structural evolution, the shear stress response of the LCP to the inception of shear, in combination with a controlled relaxation period, can provide information on the textural relaxation via the characteristics in the transient behavior ${ }^{19,44}$.

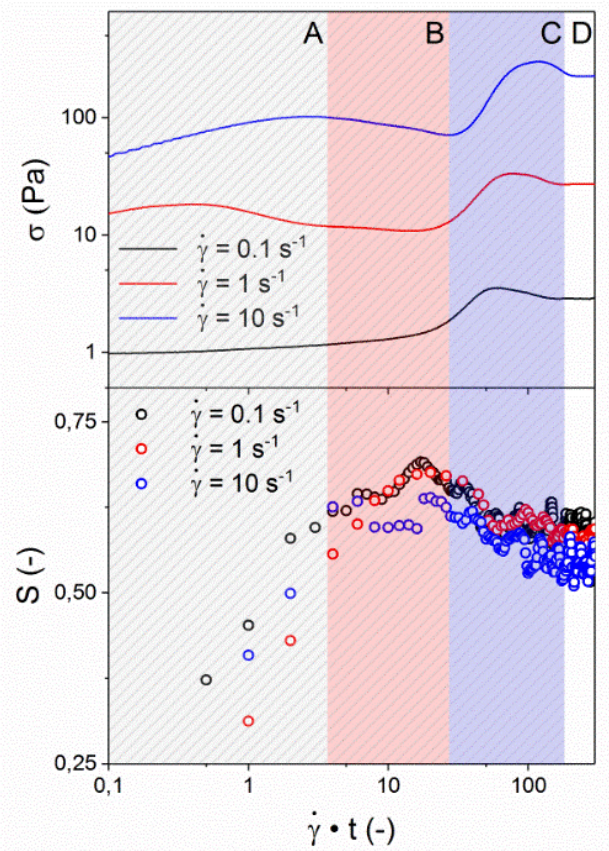

Figure 2.8 Response to inception of shear flow at $150^{\circ} \mathrm{C}$ for shear stress $\sigma$, and orientation parameter $S$. Please note, normalization of the $x$-axis has been performed to compare the shear behavior by plotting $\gamma \cdot t$. The 4 characteristic regimes found during the inception of flow are marked in the figure.

From Figure 2.8, it is apparent that the observed shear stress shows the characteristic features of a polydomain morphology at the measured shear rates, although these features are more dominant at higher shear rates. The characteristic peak in shear stress at low strain values (A) is followed by a minimum in shear stress (B), a second peak (C), and the steady-state plateau (D). All four regimes occur at the same strain, indicating that they are independent of the strain rate. The overall shear stress $(\sigma)$ scales linearly with the applied shear rate, which is expected as the steady-state viscosity is considered constant at the current shear rates, as is visible from Figure 2.7. As is depicted in Figure 
2.1, during Rheo-WAXD experiments, the molecular orientation is monitored via WAXD while the viscoelastic response of the LCP to the applied shear is simultaneously measured via the in-line rheometer. In turn, the molecular orientation is converted to the orientation parameter using equations (1)-(3). The development of the orientation parameter (S), depicted in the bottom half of Figure 2.8, shows that all samples start from similar values $(\mathrm{S} \sim 0.3)$ and show a rapid increase with strain until $\gamma \sim 10$, followed by a slight overshoot before reaching a constant value $(S \sim 0.55-0.6)$. Thus, the development of the orientation parameter scales linearly with the applied shear rate; the plateau value decreases slightly with increasing shear rate.

In the evaluated shear rate regime, both the shear stress and orientation parameter evolution were found to scale linearly with shear rate. A practical consequence of this result is that independent of the shear rate the same state of interchain orientation can be obtained by applying a sufficiently large strain. For the LCP investigated in this publication the increase in interchain orientation occurs in regime $\mathrm{A}$ and $\mathrm{B}(\gamma=0-10)$, corresponding mainly to the stretching of the polydomain texture, as is supported by POM. It was observed in POM, that at higher strains, in regime B and C $(\gamma=10-100)$, breakup and coalescence processes become more dominant, leading to a maximum in the orientation parameter according to WAXD, followed by a slight decrease in the orientation parameter. Once the polydomain texture reaches its steady state, the orientation parameter and shear stress reach their respective plateau values (regime $\mathrm{D}$, $\gamma \geq 100)$. This correlation between the evolution of the shear stress, interchain orientation and the polydomain texture is strengthened by the fact that all show the same linear scaling with shear rate.

After reaching the steady-state plateau, the shear stress and orientation parameter remain constant, until the flow is stopped. Cessation of the deformation (flow) results in relaxation: there is a decrease in the shear stress over time and the texture relaxes its thermodynamically stable state. Though reported findings in literature on LCP relaxation phenomena are not consistent, the timescale for the relaxation of the shear stress is generally faster when compared with the relaxation of both the domain- and the defect textures, and the orientation parameter. In general two distinct relaxation processes are reported, where the fast initial decrease in shear stress is associated to the relaxation of the stretched nematic domains back to their relaxed state, and the slower relaxation process is often associated with coalescence of the nematic domains in the polydomain texture ${ }^{11,18,19,45}$. For both lyotropic ${ }^{38}$ and thermotropic ${ }^{11,18,19}$ LCPs it has been reported that the timescale required for the shear stress to relax is smaller than for the dynamic moduli, the latter of which is generally considered to be related to the state of the polydomain texture. 
The relaxation of the reduced shear stress $\left(\sigma / \sigma_{0}\right)$ is depicted in Figure 2.9a. It is clear the relaxation of the shear stress is enhanced by the application of increasing shear rates. The relaxation is indeed inhomogeneous, indicating the presence of two distinct relaxation processes at all the applied shear rates. During the initial stage of relaxation, the slope is moderate. The second stage is marked by steeper decrease of the reduced shear stress over time. The first process is therefore likely the result of an elastic contribution, as could be expected from the retraction of the stretched polydomain texture. The second process is more dissipative in nature, which fits with the relaxation of the nematic texture.

a

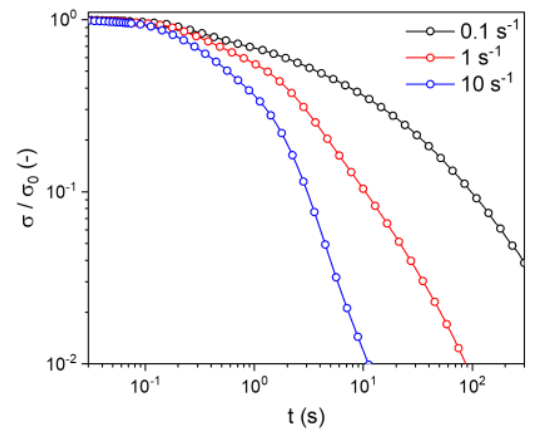

b

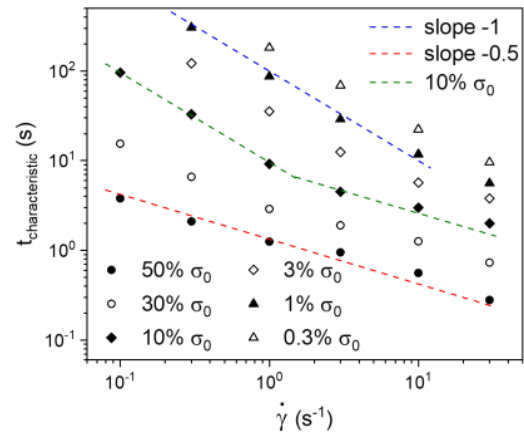

Figure 2.9 (a) Reduced shear stress as function of time for different shear rates at $150{ }^{\circ} \mathrm{C}$. A strain of at least 200 s.u. was applied to the molten LCP prior to relaxation. (b) The characteristic timescales for the relaxation of the shear stress, at different percentages of the applied shear stress $\left(\sigma_{0}\right)$, as a function of the shear rate. The data in Figure $9 b$ is obtained from shear stress relaxation experiments like those shown in Figure $9 a$. The green dotted line illustrates the bend in the curve at $10 \% \sigma_{0}$, signifying the relative contributions of both the relaxation processes at different shear rates.

The effect of shear rate on the two processes observed in the stress relaxation of the

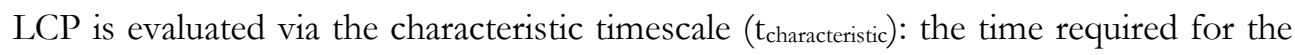
shear stress to relax to a specified percentage of the original value of the applied shear stress $\left(\sigma_{0}\right)$. The characteristic timescales confirm that two distinct processes govern the relaxation of the shear stress, as different scaling with shear rate are observed at different extents of relaxation. At smaller extents of relaxation $\left(50 \% \quad \sigma_{0}\right.$ and $\left.30 \% \quad \sigma_{0}\right)$ the characteristic timescale has a slope of -0.5 , whereas the slope shifts to -1 in the case of more extensive relaxation $\left(1 \% \sigma_{0}\right.$ and $\left.0.3 \% \sigma_{0}\right)$. The former corresponds to the initial relaxation process via contraction, whereas the latter corresponds to relaxation via coalescence and scales linearly with the applied share rate. The contraction process appears to have a larger contribution to the overall relaxation of the shear stress at low shear rates. On the high shear rate side of Figure $2.9 \mathrm{~b}$, a slope of -0.5 is found for higher 
extents of relaxation, whereas on the lower shear rate side the slope transitions to -1 at a relatively small extent of the relaxation. Visually this is observed as a bend in the trend of the characteristic time at $10 \% \sigma_{0}$ as a function of shear rate, which is marked by the green dotted line.

In order to obtain more information regarding the processes involved, the evolution of both the reduced shear stress and molecular orientation upon cessation of flow are compared for three different shear rates $\left(0.1,1\right.$, and $\left.10 \mathrm{~s}^{-1}\right)$, as depicted in Figure 2.10. The timescales are adjusted for the applied shear rate in order to illustrate the effect of the shear rate on the relaxation. The relaxation of the shear stress (Figure 2.10 a) for the different shear rates are found not to collapse on the same curve. This is expected because the relaxation of the shear rate consists of two distinct processes that scale differently with the shear rate. At short timescales, the curves for the different measurements do not overlap since the fast relaxation process does not scale linearly with shear rate, whereas at longer timescales, corresponding to the second process, which does scale linearly with the applied shear rate, the curves converge. Similar behavior has been observed for other thermotropic LCPs ${ }^{11,19}$. Interestingly, the relaxation of the orientation parameter (Figure $2.10 \mathrm{~b}$ ) is delayed, with an onset at approximately 10 (marked by the dotted line). By this time, independent of shear rate, the shear stress has relaxed significantly below $50 \%$ of the original value (Figure $2.10 \mathrm{a}$ ). The relaxation of the interchain orientation, as observed via the orientation parameter, scales linearly with the applied shear rate. Thus, the decay in the orientation parameter can be described by a single exponential decay function when the relaxation time is adjusted for the applied shear rate. The timescale of the second relaxation process (coalescence of the polydomain texture) observed for the shear stress matches well with that of the relaxation of the interchain orientation, and in both cases, linear scaling with shear rate is observed. This indicates that there exists a correlation between this relaxation process, hence the coalescence of the nematic domains in the polydomaintexture, and the orientation parameter. These observations strongly indicate that processing of LCPs at high shear rates result in faster relaxation of the orientation parameter, and potential loss of properties. In fact, such a hypothesis might provide some context to seemingly incoherent data regarding the orientation and mechanical properties of LCPs found in other studies, as will be addressed in a later section. 
Effect of shear rate on the orientation and relaxation of a vanillic acid based liquid crystalline polymer.

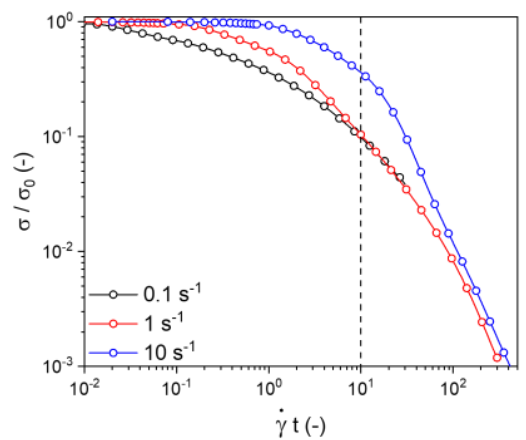

b

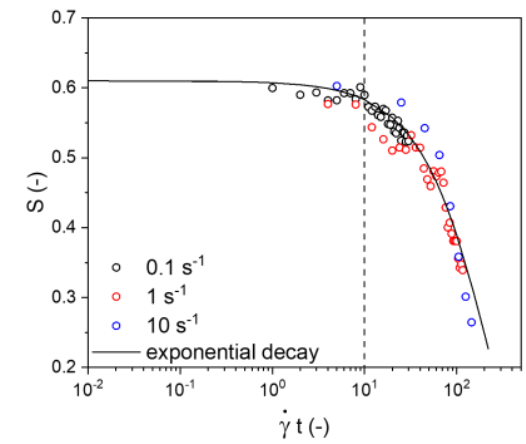

Figure 2.10 Relaxation of the investigated LCP after cessation of shear. A strain of at least 200 s.u. was applied at $150^{\circ} \mathrm{C}$ prior to relaxation. (a) Reduced shear stress as a function of time, adjusted for the applied shear rate. (b) Interchain orientation parameter as a function of time, adjusted for the applied shear rate. The dotted lines mark the onset of relaxation of the order parameter.

To probe the relaxation process of the LCP further, the melt was deformed at a shear rate of $10 \mathrm{~s}^{-1}$ until the steady state was reached, after which it was allowed to relax for a controlled amount of time (1 or $10 \mathrm{~s}$ ) before the deformation was resumed. The reinception of flow provides insight in the extent of relaxation of the domain texture ${ }^{46}$. Additionally, a flow reversal experiment was carried out, and the results are presented in Figure 2.11.
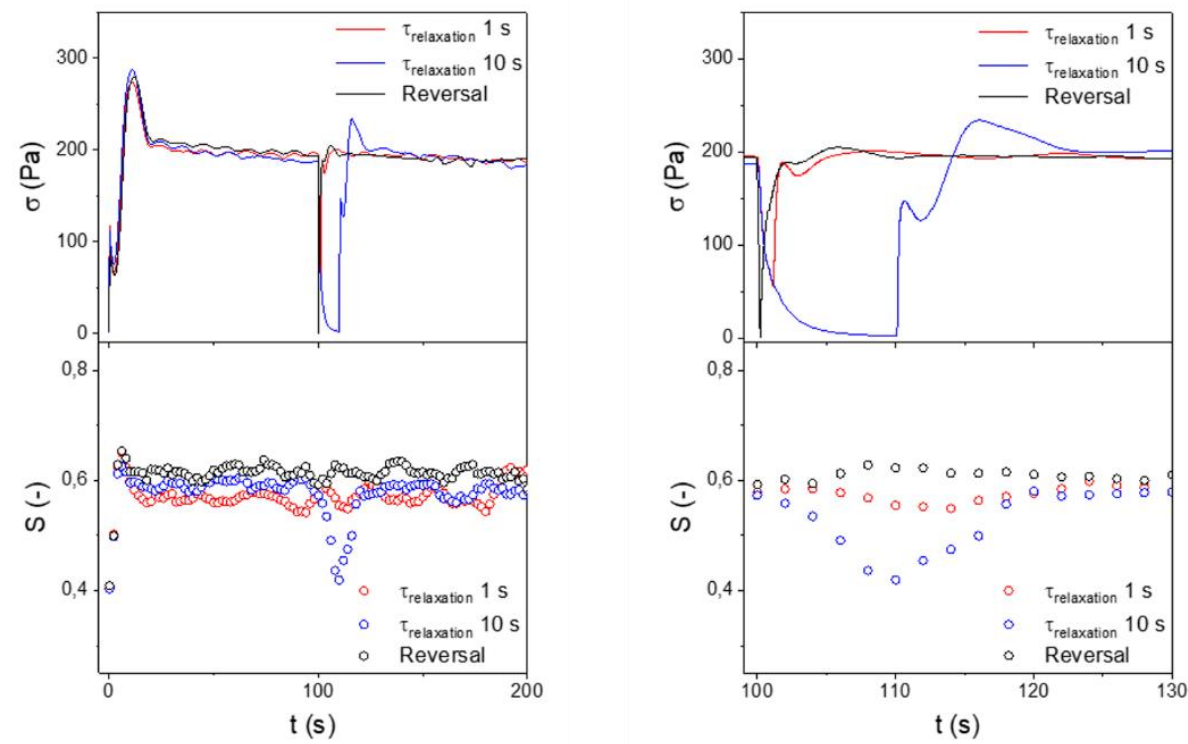

Figure 2.11 Development of shear stress and orientation parameter as a function of time for controlled relaxation experiments. The applied shear rate was $10 s^{-1}$ at $150^{\circ} \mathrm{C}$. 
In general, the shear-stress behavior of the melt during the initial flow inception showed the characteristic minimum and maximum which is in line with previous experiments performed at this shear rate, as depicted in Figure 2.8. Upon cessation of flow for $1 \mathrm{~s}$, relaxation proceeds in good agreement with the relaxation experiments, as no change in the orientation parameter was observed. The exposure time of the WAXD detector was 2 seconds, making the detection of changes on short timescales unlikely, but these findings are in good agreement with the longer relaxation experiments that were carried out at this shear rate, as shown in Figure 2.9. However, after a relaxation period of $10 \mathrm{~s}$ the value of the orientation parameter had already decreased to $\sim 0.4$, but recovered to the previous plateau value of roughly 0.57 within $10 \mathrm{~s}$ upon the reinception of shear (corresponding to $\sim 100$ strain units). During these relaxation periods, the shear stress has relaxed to a much larger extent. Upon flow reversal, no detectable change in orientation parameter was observed. Similarly, the shear stress profile during re-inception of flow provides information on the state of the polydomain texture. After a relaxation period of $1 \mathrm{~s}$ the characteristic maxima and minimum in the transient behavior of LCPs are visible, but they are very close to the plateau value. As such, relaxation has occurred primarily via contraction of the stretched texture. During the re-inception of flow after a relaxation period of $10 \mathrm{~s}$, the characteristics of the transient are significantly more pronounced, indicating that significant coalescence of the polydomain texture has occurred, corresponding with the decrease in the orientation parameter.

These findings, in combination with observations based on microscopy, provide new insight in the relaxation behavior of the LCP melt: The relaxation of the polydomain texture occurs via two distinct mechanisms being contraction of stretch, followed by coalescence. Similarly, the relaxation of the shear stress is governed by two distinct processes, with separate timescales, where the initial fast relaxation of the shear stress is caused by the dissipation of elastic energy stored in the stretched polydomain texture. According to our results, the slower shear stress relaxation process, and correspondingly the relaxation of the orientation parameter, are the result of the coalescence of the broken up domain texture.

In general, our findings are well in line with the findings on the relaxation behavior of Vectra B950, performed by Burghardt et al. ${ }^{11}$ and Beekmans et al. ${ }^{18}$. In their study, Burghardt et al. evaluated the relaxation behavior of the shear stress and the orientation parameter. These authors demonstrated that the shear stress relaxes to a large extent within a normalized timescale of 500 , but exhibited no clear decrease in the interchain orientation within the same timescales. Beekmans et al. studied the relaxation of the polydomain texture via a controlled relaxation period followed by a transient and 
demonstrated that coalescence of the polydomain texture only takes place at timescales longer than 500 units. Since the long timescales required for coalescence exceeded the experimental timescale of Burghardt et al., no consistent changes in the orientation parameter were observed, as is expected based on our findings. To recall our results show coalescence of the polydomain texture to be the governing factor for the relaxation of the orientation parameter, connecting the observations of Burghardt et al. and Beekmans et al.

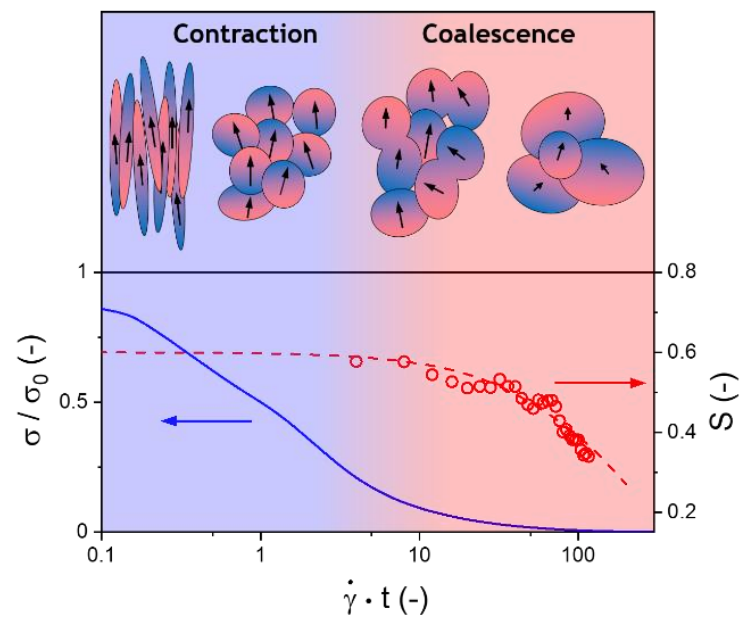

Figure 2.12 Overview of relaxation processes and timescales in LCP.

Figure 2.12 shows the schematic representation of the anticipated relaxation process according to the findings in this work. The initial contraction of the stretched domain texture results in almost complete relaxation of the shear stress. Subsequently, coalescence processes become the main contributor to the relaxation of the polydomain texture. This is a comparatively slow process, where in order for two domains to coalesce changes in interchain orientation are required at the interface of the domains. Local variations in interchain orientation arise, which as coalescence proceeds, become more significant and lead to changes on the level of the irradiated sample, resulting in a decrease in the overall orientation parameter.

The information regarding relaxation processes obtained in this study might be able to clarify a number of previous observations on the behavior of thermotropic LCPs with respect to processing and measurement conditions. As reported in the introductory section of this work, processing studies done on the PET-HBA LCP yielded a contradictory relation between the mechanical properties of the obtained fiber and the processing temperature. Acierno et al. ${ }^{6}$ found improved strength, stiffness, and interchain orientation at lowered temperatures in a process relying on an extensional 
flow field. This finding is in line with our observations; a decrease in processing temperature results in both an increase in textural and interchain relaxation time of the LCP as it exits the die. Because of the increased relaxation time, a higher orientation can be achieved with extensional flow before quenching the fiber. According to our findings, such behavior can be expected for processes where the LCP is subjected to high shear-rate conditions before exiting the die. Muramatsu and Krigbaum $^{8-10}$ found the opposite temperature dependence in a process where a shear flow field played an important role in orienting the chains. In this scenario, the increased processing temperature should increase relaxation rates after processing, unless the shear orientation is performed under well-defined and low shear rates. The application of a large amount of shear at a low shear rate effectively results in molecular orientation of the LCP but with slow relaxation rate. This would explain why Muramatsu and Krigbaum found no change in mechanical performance of the fibers when processed at $260{ }^{\circ} \mathrm{C}$ or higher; apparently, the LCP was not subjected to high shear rates in the used capillary rheometer, thereby yielding a textural morphology with a relaxation time significantly larger than the processing time. Three critical notes on this hypothesis should be placed: 1) Typical shear rates in industrial processing are higher than the rates applied in this study. Other relevant parameters, eg. pressure, were not taken into account in this study. 2) The backbone of the LCP tested in this study is considerably more flexible compared to most commercial LCPs. 3) The actual timescale over which the orientation parameter relaxes will vary strongly between different materials, depending not only on the applied shear rate, but also on parameters such as molecular weight, chemical composition and processing temperature.

\subsection{Conclusions}

The shear response of a bio-based thermotropic LCP has been studied via a combination of in situ POM and in situ WAXD. The studied shear rates correspond to Regime II in the typical three-regime flow curve, characteristically showing a polydomain texture that is strongly influenced by both the applied shear rate and the applied strain. Upon the inception of shear flow, the polydomain texture is initially stretched, while with increasing shear strain breakup and coalescence processes become increasingly important. A steady-state texture is reached around 100 to 200 strain-units, independent of the shear rate. The shear stress response exhibits the two maxima separated by a minimum, followed by a plateau, as expected for a polydomain LCP. The orientation parameter shows a strong increase at low strains, indicating a strong relation to the stretching of the polydomain texture. Interestingly, the value of the orientation parameter shows a small drop before the plateau value was reached. This drop can be 
associated with the more prevalent breakup of the polydomain texture at the applied strains. Persistent linear scaling with shear rate is found for all evaluated parameters. Monitoring the textural morphological changes during the relaxation process after shear using POM, two processes with distinct timescales were observed during the textural relaxation of the LCP melt: A rapid reversion of stretching of the polydomain texture, followed by slow coalescence of the domains. A very similar two-step process is observed for the relaxation of the shear stress. Combined with the findings from microscopy, these observations provide a strong indication that the fast initial process contributing to the shear stress relaxation is related to the release of elastic energy stored in the stretched polydomain texture; and that the second slower process is related to the coalescence of domains. More importantly, this second process appears to be connected to the relaxation of the interchain orientation. These observations are consistent with the performed flow-reversal and re-inception experiments. This has significant implications for the relation between the mechanical properties of LCPs and their processing conditions. Processing under high shear rate conditions speeds up the relaxation of interchain orientation. This might therefore limit the final mechanical performance after processing. This information might prove useful in linking seemingly contradictory reports on the processing and mechanical properties of LCPs and their blends. 


\subsection{References}

(1) Jackson, W. J. Liquid Crystal Polymers. 5. Liquid Crystalline Polyesters Containing Naphtalene Rings. Macromolecules 1983, 16 (7), 1027-1033.

(2) Jackson Jr., W. J.; Kuhfuss, H. F. Liquid Crystal Polymers - 3. Preparation and Properties of Poly(Ester Amides) From P-Aminobenzoic Acid and Poly(Ethylene Terephthalate). J. Appl. Polym. Sci. 1980, 25 (8), 1685-1694.

(3) Han, H.; Bhowmik, P. K. Wholly Aromatic Liquid-Crystalline Polyesters. Prog. Polym. Sci. 1997, 22 (7), 1431-1502. https://doi.org/10.1016/S0079-6700(96)00028-7.

(4) Liquid-Crystal Polymers, 1st ed.; Platé, N. A., Ed.; Springer: New York, 1993. https://doi.org/10.1007/978-1-4899-1103-2.

(5) Liquid Crystal Polymers: From Structures to Applications; Collyer, A. A., Acierno, D., Brostow, W., Eds.; Springer: Essex, 1992.

(6) Acierno, D.; Mantia, F. P. La; Polizzotti, G.; Ciferri, A.; Valenti, B. Ultrahigh Modulus Liquid Crystalline Polyesters. Macromolecules 1982, 15 (6), 1455-1460. https://doi.org/10.1021/ma00234a001.

(7) Chandrasekhar, S. Liquid Crystals, 2nd ed.; Cambridge University Press, 1992.

(8) Muramatsu, H.; Krigbaum, W. R. Fiber Spinning from the Nematic Melt . I . Copolyester of Poly (Ethylene Terephthalate) and p-Oxybenzoate. J. Appl. Polym. Sci. Part B Polym. Phys. 1986, 24, 1695-1711.

(9) Muramatsu, H.; Krigbaum, W. R. Fiber Spinning from the Nematic Melt. II Effect of Thermal History on Spinning of the Copolyester of Polyethylene Terephthalate and pOxybenoate. J. Appl. Polym. Sci. Part B Polym. Phys. 1987, 25, 803-816.

(10) Muramatsu, H.; Krigbaum, W. R. Fiber Spinning from the Nematic Melt. IV. Effect of P Oxybenzoate Crystallinity on the Spinning and Fiber Properties of the Copolyester of Polyethylene Terephthalate and P- oxybenzoate. J. Polym. Sci. Part B Polym. Phys. 1987, 25 (11), 2303-2314. https://doi.org/10.1002/polb.1987.090251108.

(11) Burghardt, W. R.; Brown, E. F.; Auad, M. L.; Kornfield, J. A. Molecular Orientation of a Commercial Thermotropic Liquid Crystalline Polymer in Simple Shear and Complex Flow. Rheol. Acta 2005, 44 (5), 446-456. https://doi.org/10.1007/s00397-004-0424-1.

(12) Guo, T.; Harrison, G. M.; Ogale, A. A. Transient Shear Rheology and Rheo-Optical Microstructural Characterization of a Thermotropic Liquid Crystalline Polymer. Polym. Eng. Sci. 2005, 45 (2), 187-197. https://doi.org/10.1002/pen.20265.

(13) Guo, T.; Naskar, A. K.; Harrison, G. M.; Ogale, A. A. Thermal and Shear Flow Effects on Microstructure of a Thermotropic Liquid Crystalline Polymer. Polym. Eng. Sci. 2006, 46, 1215-1222. https://doi.org/10.1002/pen.

(14) Wilsens, C. H. R. M.; Pepels, M. P. F.; Spoelstra, A. B.; Portale, G.; Auhl, D.; Deshmukh, Y. S.; Harings, J. A. W. Improving Stiffness, Strength, and Toughness of Poly $(\omega-$ Pentadecalactone) Fibers through in Situ Reinforcement with a Vanillic Acid-Based Thermotropic Liquid Crystalline Polyester. Macromolecules 2016, 49 (6), 2228-2237. https://doi.org/10.1021/acs.macromol.5b02419.

(15) Grasser, W.; Schmidt, H. W.; Giesa, R. Fibers Spun from Poly(Ethylene Terephthalate) Blended with a Thermotropic Liquid Crystalline Copolyester with Non-Coplanar Biphenylene Units. Polymer (Guildf). 2001, 42 (21), 8517-8527. https://doi.org/10.1016/S0032-3861(01)00357-3.

(16) Tjong, S. C.; Meng, Y. Z. Morphology and Mechanical Characteristics of Compatibilized Polyamide 6 - Liquid Crystalline Polymer Composites. Polymer (Guildf). 1997, 38 (18), 4609-4615. 
Effect of shear rate on the orientation and relaxation of a vanillic acid based liquid crystalline

polymer.

(17) Malik, T. M.; Carreau, P. J.; Chapleau, N. Characterization of Liquid Crystalline Polycarbonate Blends. Polym. Eng. Sci. 1989, 29 (9), 600-608. https://doi.org/DOI 10.1002/pen.760290906.

(18) Beekmans, F.; Gotsis, A. D.; Norder, B. Influence of the Flow History on Stress Growth and Structure Changes in the Thermotropic Liquid Crystalline Polymer Vectra B950. Rheol. Acta 1997, 36, 82-95.

(19) Beekmans, F.; Gotsis, A. D.; Norder, B. Transient and Steady-State Rheological Behavior of the Thermotropic Liquid Crystalline Polymer Vectra B950. J. Rheol. (N. Y. N. Y). 1996, 40 (5), 947. https://doi.org/10.1122/1.550788.

(20) Langelaan, H. C. On the Linearity of the Pressure Drop during Flow of Thermotropic LCPs in Slits and Capillaries. J. Rheol. (N. Y. N. Y). 1994, 38 (5), 1353. https://doi.org/10.1122/1.550548.

(21) Gillmor, J. R.; Colby, R. H.; Hall, E.; Ober, C. K. Viscoelastic Properties of a Model Main-Chain Liquid Crystalline Polyether. J. Rheol. (N. Y. N. Y). 1994, 38 (5), 1623. https://doi.org/10.1122/1.550562.

(22) Ugaz, V. M.; Burghardt, W. R.; Zhou, W.; Kornfield, J. A. Transient Molecular Orientation and Rheology in Flow Aligning Thermotropic Liquid Crystalline Polymers. J. Rheol. (N. Y. N. Y). 2001, 45 (5), 1029-1063. https://doi.org/10.1122/1.1389317.

(23) Zhou, W. J.; Kornfield, J. A.; Burghardt, W. R. Shear Aligning Properties of a MainChain Thermotropic Liquid Crystalline Polymer. Macromolecules 2001, 34 (11), 3654 3660. https://doi.org/10.1021/ma0018493.

(24) Zhou, W. J.; Kornfield, J. A.; Ugaz, V. M.; Burghardt, W. R.; Link, D. R.; Clark, N. A. Dynamics and Shear Orientation Behavior of a Main-Chain Thermotropic Liquid Crystalline Polymer. Macromolecules 1999, 32 (17), 5581-5593. https://doi.org/10.1021/ma990399f.

(25) Ferna, J. P.; Bello, A.; Pe, E. Parallel and Perpendicular Orientation in a Thermotropic $\begin{array}{llll}\text { Main-Chain } \quad \text { Liquid-Crystalline } & \text { 703-709. }\end{array}$ https://doi.org/10.1021/ma062312d.

(26) Fernandez-Ballester, L.; Thurman, D. W.; Zhou, W.; Kornfield, J. A. Effect of Long Chains on the Threshold Stresses for Flow-Induced Crystallization in IPP: Shish Kebabs vs Sausages. Macromolecules 2012, 45 (16), 6557-6570. https://doi.org/10.1021/ma3000384.

(27) Romo-Uribe, A.; Windle, A. H. "Log-Rolling” Alignment in Main-Chain Thermotropic Liquid Crystalline Polymer Melts under Shear: An In situ WAXS Study. Macromolecules 1996, 29 (19), 6246-6255. https://doi.org/10.1021/ma960211h.

(28) RomoUribe, A.; Lemmon, T. J.; Windle, A. H. Structure and Linear Viscoelastic Behavior of Main-Chain Thermotropic Liquid Crystalline Polymers. J. Rheol. (N. Y. N. Y). 1997, 41 (5), 1117-1145. https://doi.org/10.1122/1.550820.

(29) Cocchini, F.; Nobile, M. R.; Acierno, D. Transient and Steady Rheological Behavior of the Thermotropic Liquid-Crystal Copolymer 73/27 Hba Hna. J. Rheol. (N. Y. N. Y). 1991, 35 (6), 1171-1189. https://doi.org/Doi 10.1122/1.550170.

(30) Burghardt, W. R.; Fuller, G. G. Transient Shear Flow of Nematic Liquid Crystals: Manifestations of Director Tumbling. J. Rheol. (N. Y. N. Y). 1990, 34 (6), 959-992. https://doi.org/10.1122/1.550151.

(31) Wilsens, C. H. R. M.; Verhoeven, J. M. G. A.; Noordover, B. A. J.; Hansen, M. R.; Auhl, D.; Rastogi, S. Thermotropic Polyesters from 2,5-Furandicarboxylic Acid and Vanillic Acid: Synthesis, Thermal Properties, Melt Behavior, and Mechanical Performance. Macromolecules 2014, 47 (10), 3306-3316. https:// doi.org/10.1021/ma500433e. 
(32) Wilsens, C. H. R. M.; Deshmukh, Y. S.; Liu, W.; Noordover, B. A. J.; Yao, Y.; Meijer, H. E. H.; Rastogi, S. Processing and Performance of Aromatic-Aliphatic Thermotropic Polyesters Based on Vanillic Acid. Polym. (United Kingdom) 2015, 60, 198-206. https://doi.org/10.1016/j.polymer.2015.01.045.

(33) Stellamanns, E.; Meissner, D.; Lohmann, M.; Struth, B. A Unique Rheology / SAXS Combination at DESY / Petra III. J. Phys. Conf. Ser. 2013, 425 (PART 20), 18-21. https://doi.org/10.1088/1742-6596/425/20/202007.

(34) Mitchell, G. R.; Windle, A. H. Orientation in Liquid Crystal Polymers. In Developments in crystalline polymers; Basset, D. C., Ed.; Elsevier Applied Science: London, UK, 1988. https://doi.org/10.1007/978-94-009-1341-7_3.

(35) Li, X.; Huang, M.; Guan, G.; Sun, T. Molecular Structure of Liquid-Crystalline Copolyesters of Vanillic Acid , 4-Hydroxybenzoic Acid and Poly ( Ethylene Terep h Thalate ). Die Angew. Makromol. Chemie 1995, 227, 69-85.

(36) Vermant, J.; Moldenaers, P.; Picken, S. J.; Mewis, J. A Comparison between Texture and Rheological Behavior of Lyotropic Liquid-Crystalline Polymers during Flow. J. Nonnewton. Fluid Mech. 1994, 53, 1-23. https://doi.org/10.1016/0377-0257(94)85039-9.

(46) Viola, G. G.; Baird, D. G. Studies on the Transient Shear Flow Behavior of Liquid Crystalline Polymers. J. Rheol. (N. Y. N. Y). 1986, 30 (3), 601-628. https://doi.org/10.1122/1.549863. 


\title{
Chapter 3 - Controlling processing, morphology and mechanical performance in blends of polylactide and thermotropic polyesters.
}

\begin{abstract}
Thermoplastic composites based on thermotropic liquid crystalline (LCP) materials are interesting candidates for reinforced composite application due to their promising mechanical performance and potential for recyclability. In combination with a societal push towards the more sustainable use of materials, these properties warrant new interest in this class of composites. Though numerous studies have been performed in the past, a coherent set of design rules for LCP design for the generation of injection molded reinforced thermoplastic composites is not yet available, likely due to the complex interplay between LCP and matrix components. In this study, we report on the processing of poly(L-lactide) (PLLA) with two different LCPs, with low processing temperatures, to identify critical parameters for the morphological development and mechanical performance of LCP reinforced composites. The influence of blend composition and the processing conditions on mechanical response of the composites is investigated using rheology, Wide-angle X-ray Diffraction, mechanical analysis, and microscopic techniques. In general, we observe that both the matrix viscosity and the viscosity ratio are determining the deformation and breakup of dispersed LCP droplets during extrusion processes. In addition, their thermal dependency appears to be a critical parameter for the final composite performance during injection molding: stretching and molecular orientation of the LCP phase into highly oriented fibrils is prevented when the viscosity ratio increases rapidly upon cooling. In contrast, melt drawing proves to be a more effective processing route as the extensional flow field stabilizes elongated droplets regardless of the viscosity ratio. Overall, these findings provide valuable insight in the morphological development of LCP reinforced blends, highlight the importance of the development of viscoelastic properties as a function of temperature, and provide guidelines for the design of new LCP polymers and their thermoplastic composites.
\end{abstract}

Published as:

de Kort, G. W.; Rastogi, S.; Wilsens, C. H. R. M. Controlling Processing, Morphology, and Mechanical Performance in Blends of Polylactide and Thermotropic Polyesters. Macromolecules, 2019, 52, 6005-6017. 


\subsection{Introduction}

In the $21^{\text {st }}$ century, a societal push towards the more sustainable use of materials has developed. Fiber-reinforced composites have excellent mechanical properties, and are used in a wide range of applications; However, the end-of-life options for these materials are often lacking sustainability. ${ }^{1-3}$ In situ formed composites based on mainchain thermotropic liquid crystalline polymers (LCPs) and a thermoplastic matrix have the potential to serve as a more sustainable type of reinforced composites. Though promising, the mechanical properties of these in situ composites are often in the same range as short glass fiber-reinforced composites 4,5 , that have a Young's modulus up to $10 \mathrm{GPa}$ and can sustain stresses up to $150 \mathrm{MPa}$. ${ }^{6,7}$ They are, however, unlikely to match those of composites based long or continues fibers (eg. carbon fiber, or premade fibers of high melting or lyotropic LCPs), in which the reinforcing fibers can have a Young's modulus over $100 \mathrm{GPa}$ and a strength over $1 \mathrm{GPa} .{ }^{8,9}$ However, in situ LCP composites are suitable reinforced materials that are compatible with conventional processing techniques such as extrusion and injection molding, making them versatile in terms of design, cheap in manufacturing, and candidates for thermomechanical recycling., $4,5,10$ These properties, in combination with a drive towards sustainability, and advances in LCPs and matrix materials, warrant renewed interest in this type of composites.

Most commercially available LCPs having a high characteristic ratio, are fully aromatic in nature, and have high melting and processing temperatures $\left(>280^{\circ} \mathrm{C}\right)$. This poses a limitation in blending because of the limited thermal stability of most flexible thermoplastic polymers. Therefore, high melting LCPs are normally blended with thermally stable thermoplastic polymers such as PET, and PBT. ${ }^{4,10-13}$ The processing temperatures of LCPs can be suppressed by the inclusion of irregular and/or flexible comonomers along the rigid backbone. ${ }^{14-16}$ Given the lower thermal requirements of these LCPs, they are usable for the generation of thermoplastic composites based on renewable and thermally labile polymers, including Poly(L-lactide) (PLLA), poly(butylene succinate) and poly(w-pentadecalactone). ${ }^{17-19}$

PLLA is a suitable matrix for LCP reinforced composites, and is among the frontrunners of bio-based plastics, is produced on commercial scale, and is used in a myriad of applications ${ }^{20}$. With its glass transition temperature approximately $60{ }^{\circ} \mathrm{C}$, PLLA is characterized as a strong, brittle polymer at room temperature. Although it is classified as a semi-crystalline polymer, the slow crystallization kinetics often result in amorphous products. It is biocompatible, and under the right conditions, biodegradable. These properties, in combination with strong, biodegradable LCPs ${ }^{21-23}$ 
opens up a pathway to reinforced composite materials that are not only reprocessable, but also fully biodegradable or even biocompatible.

The reinforcing effect in such LCP/PLLA composites is resulting from the excellent physical and mechanical properties of the $\mathrm{LCPs}^{24-27}$. In particular, the nematic phase in LCPs invokes low melt viscosities, complex rheological behavior ${ }^{28-30}$, and an ease in chain orientation, which, when maintained on cooling, results in products having a high degree of molecular orientation and the desired mechanical reinforcement. However, as is reported by for example Kiss et al., Blizard et al., and Song and Isayev, the mechanical properties of the LCP composites are found to be strongly dependent on the LCP morphology and interchain orientation, where both parameters are influenced by the applied processing conditions $\mathbf{4}^{4,10-13,16,24,31}$. For example, during extrusion, the LCP phase is dispersed in the thermoplastic matrix whereas the particle size is governed by the viscosity ratio (the LCP viscosity divided by matrix viscosity). ${ }^{32}$ The generated particles need to be deformed to obtain the desired high interchain orientation. This is generally done by subjecting the blend to shear flow, elongation flow or a combination thereof. As is reported by for example Heino and coworkers ${ }^{32}$, flow fields that contain a strong elongational component, for example in fiber spinning, are very effective at deforming the particles to yield the desired morphology. In contrast, LCP particle deformation in flow fields with a dominant shear component, for example in injection molding, is not as straightforward as the effective deformation relies on the interplay between the viscosity ratio, the generated particle size, and thermal behavior of both constituents. ${ }^{32-}$ 36 Though the aforementioned studies have clearly demonstrated that LCPs can be effective reinforcing fillers, a coherent set of design rules for LCP materials for use in reinforced thermoplastic composites for injection molding is not yet available, likely resulting from the complex interplay between LCP and matrix components.

To identify critical LCP properties required for effective LCP reinforcement in shearflow fields, we report on the influence of the LCP flow behavior and its thermal dependence on the morphological development in LCP/PLLA blends during and after processing. To be more precise, we evaluate the behavior of two different LCPs which can be processed at relatively low temperatures; The first LCP is the commercially available, aromatic copolymer Vectra ${ }^{\circledR}$ LCP V400P (LCP-A), whereas the other is an in-house synthesized semi-flexible LCP (LCP-B) having both aliphatic and aromatic comonomers. Relaxation occurs significantly faster in the case of LCP-B compared to its aromatic counterpart. Furthermore, the morphological development of the LCP components in the PLLA matrix is assessed during processing via two different processing routes: injection molding route, having a dominant shear-flow component (Figure 3.1a) and a melt-drawing route having a predominant elongation-flow field 
(Figure 3.1b). Lastly, the mechanical performance of the generated products is evaluated and correlated to the orientation parameter determined through Wide-angle X-ray Diffraction. The generated findings are used to identify structural characteristics required in LCPs for use as reinforced thermoplastic LCP composites made via injection molding.

a

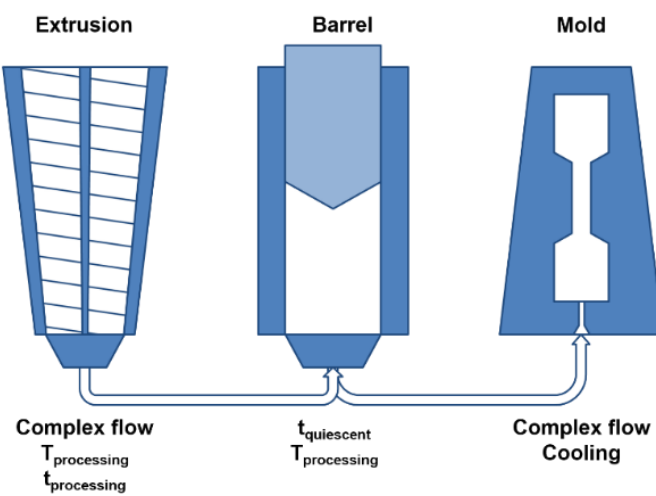

b Extrusion Drawing

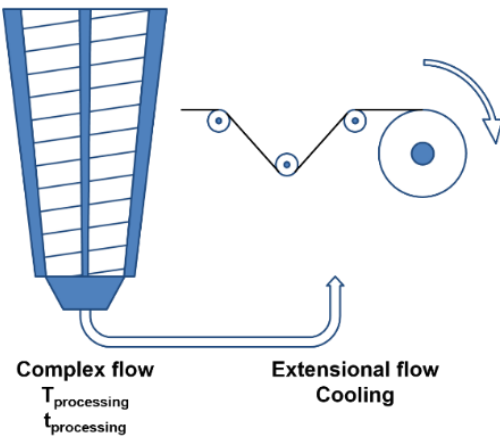

Figure 3.1 Processing routes applied in this study: (a) injection molding and (b) melt-drawing.

\subsection{Methods and Materials}

\section{Materials}

Suberic acid, 1,4-diacetoxybenzene, and p-acetoxybenzoic acid was purchased from TCI Europe. The PLLA used in this study was purchased from Corbion (grade L130). The commercial Vectra ${ }^{\circledR}$ LCP V400P was purchased from Celanese. Vectra ${ }^{\circledR}$ LCP V400P grade is a registered trademark of Celanese Corporation, Dallas, TX. The material composition as well as the results associated with this material are the property of Celanese Corporation.

\section{Polymerization Procedure of LCP-B}

The synthesis of semi-flexible LCP-B was performed based on a previously described procedure: a $1000 \mathrm{~mL}$ three-neck glass vessel fitted with a mechanical stirrer. The monomer mixture consisting of p-acetoxybenzoic acid (164.2 g, $911 \mathrm{mmol})$, suberic acid (158.8 g, $911 \mathrm{mmol})$, 1,4-diacetoxybenzene $(177.0 \mathrm{~g}, 911 \mathrm{mmol})$ was introduced together with $300 \mathrm{mg}$ of $\mathrm{Zn}(\mathrm{OAc})_{2}$ to the round-bottom flask. The monomers were dried overnight in vacuo at $60^{\circ} \mathrm{C}$ prior to usage to eliminate moisture. Furthermore, after loading of the monomers the round-bottom flask was iteratively flushed with nitrogen and reduced pressure for three times prior to the start of the reaction to minimize oxygen content. Next, a small nitrogen flow was applied to the system and the 
temperature was increased stepwise to $200{ }^{\circ} \mathrm{C}$. As soon as acetic acid started to be formed, the reaction temperature was gradually increased to $240^{\circ} \mathrm{C}$ after which the polymerization was allowed to proceed for $6 \mathrm{~h}$. Next, reduced pressure was applied to the system for $12 \mathrm{~h}$ to build up molecular weight. The final product was isolated from the hot reactor flask in the form of a polymer melt.

\section{Preparation and Processing of PLLA-LCP Blends}

PLLA and the LCPs were dried overnight $24 \mathrm{~h}$ at $60^{\circ} \mathrm{C}$ in vacuo prior to use. Mixtures of polymer pallets were prepared in the following compositions: $0 \mathrm{wt} \%$ LCP (i.e. pure PLLA), $10 \mathrm{wt} \%$ LCP, and $30 \mathrm{wt} \%$ LCP. Next, the mixtures were blended in a DSM Xplore twin-screw micro-extruder with a barrel size of $5 \mathrm{ml}$. This micro-extruder has a recycle channel and allows for circulation of the material for a given time before guiding the material to the extruder exit using a valve. The materials were mixed for 3 minutes at $100 \mathrm{rpm}$ at a processing temperature of $240{ }^{\circ} \mathrm{C}$ for pure PLLA and LCP-A/PLLA blends and $220^{\circ} \mathrm{C}$ for LCP-B/PLLA blends, respectively. After blending, the samples were either processed directly into tapes or transferred into a hot barrel and injection molded into tensile bars. Tapes were generated using a slit die mounted at the extruder outlet ( $0.5 \times 3 \mathrm{~mm}$, produced by DSM Xplore) and a winder (Dienes SD-type). For the generation of the tapes, the extrusion rate was set at $10 \mathrm{rpm}$ and the winder was set at $20 \mathrm{rpm}$. The tapes were drawn and cooled in air. A DSM Xplore IM 5.5 micro injection molder was used to produce tensile bars $(2 \mathrm{~mm} \times 4 \mathrm{~mm} \times 70 \mathrm{~mm}$, with a gage length of $25 \mathrm{~mm}$ ). The barrel temperature was set to the previously used processing temperature for the respective composition, while the mold temperature was set to $25^{\circ} \mathrm{C}$.

\section{Material Characterization.}

The viscoelastic behavior of the blend components was determined in a TA Instruments Discovery HR 2 rheometer with a parallel plate geometry (diameter of 25 $\mathrm{mm}$, gap of $1 \mathrm{~mm}$ ). Samples were loaded at $200{ }^{\circ} \mathrm{C}$ and subsequently heated or cooled at a rate of $5{ }^{\circ} \mathrm{C} \mathrm{min}^{-1}$ to the required temperature for a frequency sweep at a strain of $1 \%$. The intrinsic relaxation behavior of the LCPs was probed with the same rheometer, at the respective processing temperatures. The specified shear stress was applied to the sample until a strain of at least 300 s.u. was reached (within the plateau region). Next, the deformation was ceased for a given relaxation period after which the stress was reapplied. This second transient was used to acquire information regarding the respective relaxation behavior of the LCPs. The experiments probing the intrinsic relaxation behavior were carried out at the respective processing temperature of each LCP. 
The glass transition temperature $\left(T_{\mathrm{g}}\right)$ and the peak melting temperature $\left(T_{\mathrm{m}}\right)$ were determined by differential scanning calorimetry (DSC) using a TA Instruments Q2000 DSC. The heating and cooling-rates of the sample were $10{ }^{\circ} \mathrm{C} \mathrm{min}^{-1}$ and measurements were performed under a nitrogen rich atmosphere.

The blend morphology was evaluated via polarized optical microscopy (POM) using an Olympus BX53 Microscope (20 or 50 times magnification) equipped with an Olympus DP26 camera and a $530 \mathrm{~nm}$ retardation plate. To display the LCP particle morphology created in each processing step, the PLLA-phase was dissolved in a mixture of acetone and dichloromethane (3:1 by volume) in which neither of the used LCPs is soluble.

Scanning electron microscopy (SEM) was performed on a Philips XL30 system at an acceleration voltage of $15 \mathrm{kV}$ using 1000 times magnification. The freshly prepared fracture surfaces were attached to the sample holder with conductive carbon tape and subsequently coated with a thin layer of gold.

Tensile testing performed on a Zwick Z100 tensile on both tapes and tensile bars. Samples were subjected to a constant deformation rate of $5 \mathrm{~mm} \mathrm{~min}^{-1}$, at room temperature. A $10 \mathrm{kN}$ load-cell was used for the injection molded bars. For the tapes, a length of $5 \mathrm{~cm}$ was tested via a $200 \mathrm{~N}$ load-cell.

The molecular orientation of the LCP phase in the pure LCP samples and in the blends was assessed via 2D Wide-angle X-ray Diffraction (WAXD) using a SAXSLAB Ganesha diffractometer using $\mathrm{Cu} \mathrm{K}-\alpha$ radiation $(\lambda=0.154 \mathrm{~nm})$. The beam center and $\theta$-range were calibrated via the diffraction pattern of silver behenate. The orientation parameter $(\mathrm{S}),\left\langle\mathrm{P}_{2 \mathrm{n}}(\cos \varphi)>_{\mathrm{d}}\right.$, was calculated from the obtained diffraction patterns via a procedure by Mitchell and Windle ${ }^{37}$, described in Chapter 2.

\subsection{Theoretical considerations}

In general, the morphological development of blends during processing can qualitatively be analyzed within the framework of breakup and coalescence processes. This theory, based on Einstein's work on the viscosity of dilute suspensions ${ }^{38,39}$, was extended to emulsions by Taylor ${ }^{40,41}$ whereas Oldroyd ${ }^{42,43}$ later incorporated the effects of interfaces. Although this framework was originally developed for systems subjected to low deformation rates and for emulsions of Newtonian liquids, it has also provided insight in complex systems involving viscoelastic dispersed- and matrix phases ${ }^{44-47}$. Therefore, these theoretical concepts are used to describe the morphological development during the processing of the thermoplastic polymers in this study. Buoyancy is not taken into account in this theory, which is considered a reasonable assumption for polymer melts with high viscosities. 
The deformation of a spherical liquid droplet in a matrix can be described mainly by two dimensionless numbers: the capillary number $(\varkappa$, Eq. 1$)$ and the viscosity ratio $(\lambda$, Eq. 2).

$$
\begin{gathered}
\kappa=\frac{\eta_{\text {matrix }} \cdot \dot{\gamma} \cdot d}{v_{12}} \\
\lambda=\frac{\eta_{\text {droplet }}}{\eta_{\text {matrix }}}
\end{gathered}
$$

In these equations, $\dot{\gamma}$ represents the applied shear rate, $\mathrm{d}$ represents the droplet diameter, $v_{12}$ represents the interfacial tension, and $\eta$ represents the viscosity of either the dispersed or the matrix phase. The capillary number describes the balance of hydrodynamic- and surface forces acting on a dispersed droplet with a specific diameter. The viscosity ratio is a measure of how effectively such a droplet can be deformed by the matrix.

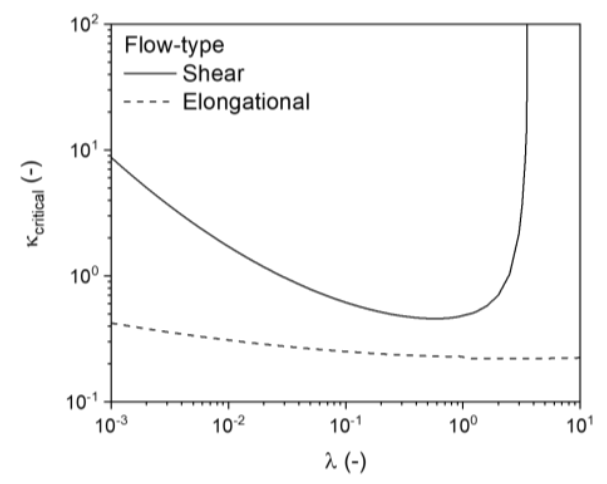

Figure 3.2 Critical capillary number (xeritial) as a function of viscosity ratio ( $\lambda$ ).

The type of flow field to which an emulsion is subjected has a strong influence on the behavior of droplets, as was shown by Tomotika ${ }^{48}$. In order to allow droplet deformation, the capillary number needs to be larger than a certain value: the critical capillary number ( $x_{\text {critical }}$, Eq. 3). As the droplet stretches, its diameter, and correspondingly the capillary number, decreases. When the capillary number of a droplet equals the critical value, the hydrodynamic forces no longer stabilize the deformed droplet and breakup can occur. Values for $\chi_{\text {critical }}$ in different types of flow fields have been experimentally determined ${ }^{44,49}$ as a function of $\lambda$ (Figure 3.2). The constants $c_{1}-c_{5}$ in Eq. 3 are flow-type specific and are provided in the Supporting Information.

$$
\begin{gathered}
\log \left(\kappa_{\text {critical }}\right)=c_{1}+c_{2} \cdot \log (\lambda)+c_{3} \cdot \log (\lambda)^{2}+\frac{c_{4}}{\log (\lambda)+c_{5}} \\
\kappa^{*}=\frac{\kappa}{\kappa_{\text {critical }}}
\end{gathered}
$$


Based on Figure 3.2, one can deduce that the application of shear-flow is not sufficient to disperse droplets with a high viscosity ratio $(\lambda>3.8)$. In this scenario, the timescale for deformation becomes so large that the droplets simply rotate. In contrast, an extensional flow field can deform droplets regardless of viscosity ratio ${ }^{50,51}$, and is more effective in deforming and breaking up of droplets compared to shear flow.

The behavior of a droplet in a matrix can be described by the reduced capillary number, $x^{*}$, (Eq. 4), being the ratio of the capillary number $x$ and the critical capillary number $x_{\text {critical. }}$. Four regimes in droplet behavior can be identified with respect to $x^{* 52}$. Regime 1 involves the scenario where $x^{*}<0.1$, a regime where no deformation of droplets occurs in a matrix. Regime 2 corresponds to $0.1>x^{*}>1$, corresponding to deformation of droplets without breakup. Regime 3, occurring when $1>x^{*}>4$ corresponds to the deformation of droplets and their splitting into primary droplets. Lastly, affine droplet deformation into stable filaments occurs in regime 4 , when $x^{*}>4$. To illustrate, a large droplet, having a $x^{*}$ corresponding to regime 3 , will deform and break up when subjected to a flow field, effectively decreasing its diameter and thus its capillary number $x$. This reduces both the capillary number $x$ and reduced capillary number, $x^{*}$. This continues until $x^{*}$ becomes smaller than 1 , effectively reaching regime 1 or regime 2 where no further breakup occurs.

In order for the droplets to behave in this manner, the forces involved need to provide a certain amount of work. As a result, the scale of the deformation and the time over which it occurs are relevant for the behavior of droplets. The timescale associated with complete breakup of a droplet ( $t^{*}$, Eq. 5) was determined experimentally $y^{49,53}$ :

$$
t^{*} \cong 84 \cdot \lambda^{0.355} \cdot \kappa^{*-0.599}
$$

The theoretical approach so far describes the behavior of individual droplets, but large amounts of droplets are present in real systems. These droplets can collide, and in case the fluid matrix separating the particles is drained, the droplets can coalesce again forming larger droplets. Because of coalescence, the observed droplet size is generally larger than predicted from theories that only account for particle breakup (with very dilute systems as exception) ${ }^{54}$. Many parameters that promote breakup, influence the coalescence of droplets in a similar fashion: higher shear rates and a lower viscosity ratio tend to accelerate coalescence ${ }^{55}$. The number of droplets present in a volume, the draining conditions, and the timescale of contact between different droplets govern the overall coalescence process. More importantly, the opposing effects of breakup and coalescence determine the dynamic equilibrium state for a constant flow field that is applied on a sufficient timescale. Under such conditions, though the system remains dynamic, the overall morphology of the blend remains constant. 
The theoretical framework described above was initially developed to describe Newtonian systems. However, the rise of polymer blending, which involves viscoelastic components, required information on the breakup and deformation behavior of droplets in non-Newtonian systems. Studies have been done to determine the influence of viscoelasticity on the equilibrium blend morphology and the development of the morphology in case either the droplet phase, or the matrix phase, or both are viscoelastic ${ }^{30,44,46}$. Generally, it was found that increased elastic contributions hindered breakup and led to a larger equilibrium droplet diameter. To estimate the importance of viscoelasticity in the used blends, the Weissenberg number (Wi, Eq. 6) was calculated for the PLLA matrix at the processing temperature.

$$
W i=\frac{\text { elastic forces }}{\text { viscous forces }}=\lambda_{\text {relaxation }} \cdot \dot{\gamma}
$$

$\lambda_{\text {relaxation }}$ indicates the relaxation time of the melt, for which the reciprocal value of the crossover frequency was taken (master curve is provided in the Supporting Information), and $\dot{\gamma}$ indicates the applied shear rate (a value of $300 \mathrm{~s}^{-1}$ was taken). Values for Wi vary from 0.279 to 0.129 for processing temperatures between 220 to $240{ }^{\circ} \mathrm{C}$ respectively, indicating that under these conditions the response is mainly viscous, but that the elastic component does play a significant role. This would suggest that the experimental particle size is likely to be larger than predicted by the theory described above. Irrespective, as the development of the morphology is governed by deformation, breakup and coalescence processes, this theoretical framework will be a valuable tool to describe the morphological behavior of the blends evaluated in this study. For further information on the topic of polymer blends and their morphology the authors refer to an overview on the topic by Kamal56, and to works implementing this framework in extrusion (Shi, Utracki et al.52,54,55,57), and fiber spinning (Song and Isayev ${ }^{31}$ ).

\subsection{Results and Discussion}

\section{Thermal behavior and viscoelastic response of the blend constituents}

Prior to evaluating the effect of the processing conditions on the blend morphology and the resulting mechanical properties, the thermal and rheological behavior of the individual components was investigated. In this study, two LCPs with low processing temperatures were evaluated as reinforcing fillers for PLLA. As mentioned in the experimental section, a fully aromatic copolymer, Vectra LCP V400P (LCP-A) was used and a semi-flexible LCP was developed in-house with $33 \mathrm{~mol} \%$ of suberic acid as nonaromatic units (LCP-B). The behavior of all used polymers, as observed in DSC analysis at a heating and cooling rate of $10{ }^{\circ} \mathrm{C} \mathrm{min}^{-1}$ is shown in Figure 3.3. The heating run of 
the semi-flexible LCP-B shows a broad glass transition temperature $\left(T_{\mathrm{g}}\right)$ around $50{ }^{\circ} \mathrm{C}$, followed by a broad melting peak with a maximum at $185^{\circ} \mathrm{C}$. The broadness of the melting peak is expected to originate from the various possible crystalline aromatic sequences. When heated beyond $200^{\circ} \mathrm{C}$ the LCP-B exhibits a stable nematic phase until degradation sets in well above $300^{\circ} \mathrm{C}$, as is reported in earlier work ${ }^{58}$. LCP-A is a fully aromatic copolymer, which does not exhibit extensive formation of non-periodic layer crystallites. From Figure 3.3 it can be observed that the rigid backbone leads to a high $T_{\mathrm{g}}$ around $110{ }^{\circ} \mathrm{C}$ and a very small melting endotherm between 200 and $230^{\circ} \mathrm{C}$. Lastly, the used PLLA grade crystallizes slowly, as indicated by the incomplete crystallization during cooling and the presence of a large cold crystallization peak during heating. Additionally, the PLLA exhibits a $\mathrm{T}_{\mathrm{g}}$ around $60^{\circ} \mathrm{C}$ and a peak melting temperature of $175^{\circ} \mathrm{C}$. Overall, this data suggests that blending of PLLA with LCP-B can be performed at $220{ }^{\circ} \mathrm{C}$ as the LCP-B is fully molten at this temperature and resides in the nematic phase. Similarly, blending LCP-A in the nematic phase with PLLA and would require a temperature of $240{ }^{\circ} \mathrm{C}$.

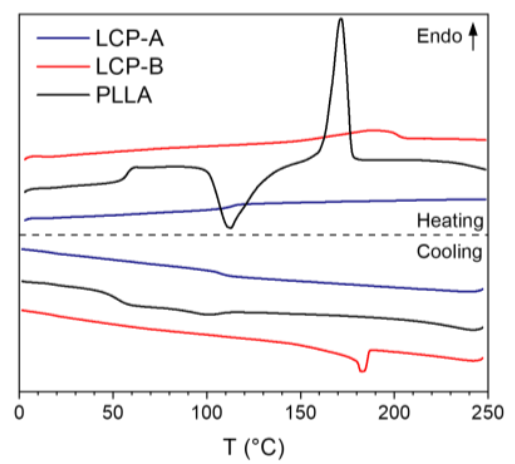

Figure 3.3 Differential scanning calorimetry thermograms of the components used in this study, taken at a beating and cooling rate of $10^{\circ} \mathrm{C} \mathrm{min}^{-1}$ (endo up).

Figure 3.4a shows the complex viscosity as a function of angular frequency for both LCPs and the PLLA at the previously mentioned processing temperatures. As expected for linear polymers, all materials show shear thinning behavior at $\omega>50 \mathrm{rad} \mathrm{s}^{-1}$. A considerable difference in viscosity between the two LCPs is observed over the measured frequency range, indicating that the viscosity ratio for the LCP-A/PLLA and LCP-B/PLLA blends are different under the used processing conditions. In drawing and injection molding, the blends are simultaneously deformed and cooled; therefore, the viscosity of the components as a function of temperature is highly relevant. Figure $3.4 \mathrm{~b}$ shows the dependency of the complex viscosity over temperature at $\omega=100 \mathrm{rad}$ $\mathrm{s}^{-1}$. One can observe that the PLLA is considerably more viscous than both LCP 
polymers above $200{ }^{\circ} \mathrm{C}$, i.e. under the used processing conditions. During cooling, the complex viscosity increases for all components. The viscosity of LCP-A surpasses that of the PLLA around $180^{\circ} \mathrm{C}$, likely resulting from the higher $T_{\mathrm{g}}$ of LCP-A compared to PLLA. For LCP-B, crystallization proceeds during cooling below $200{ }^{\circ} \mathrm{C}$, resulting in a steep increase in viscosity during further cooling. One can imagine that when blended, the differences in thermal behavior of the individual components will affect the blend morphology during processing; A change in viscosity ratio occurs during cooling, affecting the (reduced) capillary number and the particle deformation and breakup processes and thus the blend morphology.

a

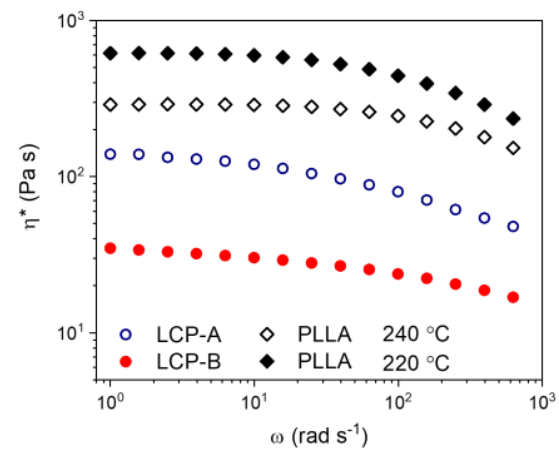

b

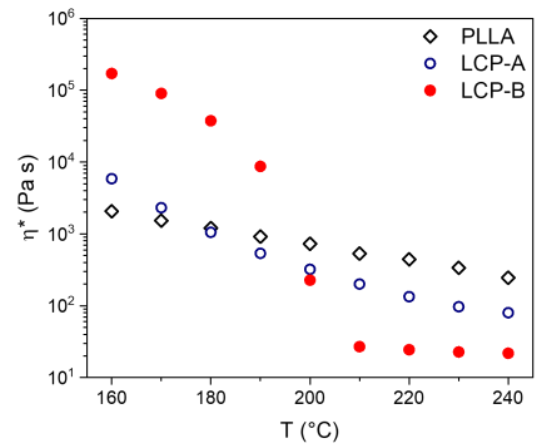

Figure 3.4 (a) Complex viscosity $\left(\eta^{*}\right)$ as a function frequency ( $\omega$ ) for PLLA, LCP-A, and LCP-B determined at the extrusion temperature of the blend components. (b) Complex viscosity of the same components as function of temperature, taken at frequency of $100 \mathrm{rad} \mathrm{s}^{-1}$.

\section{Effect of blend extrusion on LCP particle morphology}

PLLA and both LCPs were extruded for three minutes at $100 \mathrm{rpm}$ to disperse the LCP phase in the PLLA matrix. As mentioned earlier, processing temperatures for blends containing LCP-B were $220^{\circ} \mathrm{C}$, whereas blends with LCP-A were extruded at $240{ }^{\circ} \mathrm{C}$. As is depicted in Figure 3.5, the viscosity ratios at the processing temperature for the both blends are relatively constant with respect to the angular frequency. Though the shear rates can be higher during extrusion, we expect that the viscosity ratio remains constant under the used conditions. However, one can observe that the viscosity ratio for the LCP-A/PLLA blends is roughly a factor 10 larger compared to the LCP-B/PLLA blends. Despite this difference in viscosity ratio, the calculated critical capillary numbers for systems with these viscosity ratios are very similar, with $x_{\text {critical }}$ (shear) $0.5-0.75$ and $x_{\text {critical }}$ (elongation) $\sim 0.25$. In order to make an estimate for the actual value of the capillary number, a calculation was done based on a hypothetical but representative case of a droplet in a matrix with $\mathrm{d}=100 \mu \mathrm{m}, \eta_{\text {matrix }}=100 \mathrm{~Pa} \mathrm{~s}, \dot{\gamma}=100$ 
$\mathrm{s}^{-1}$, and $\nu_{12}=10 \mathrm{mN} \mathrm{m}^{-1}$. These assumptions yield a $x$ value of 100 . Consecutively, for both blends $x_{\text {critical }}<1$, regardless of the flow type, yielding a $x^{*}$ well over 100 . Based on this estimate, LCP droplets with a diameter in the micrometer range are effectively deformed by the flow field and are expected to stretch into stable filaments already during extrusion. Similarly, the timescale of droplet break up can be estimated using $\lambda$ $=0.5$ and $x^{*}=100$ (a case similar to a large droplet in a LCP-A/PLLA blend), yielding a $t^{*}$ of $4.1 \mathrm{~s}$. This value is considerably shorter than the extrusion time, confirming that droplet breakup occurs during extrusion. Note, a constant value was taken for the interfacial tension of both blends. This was done as the interfacial tension for actual polymer-polymer systems is generally in the range of $1-10 \mathrm{mN} \mathrm{m}^{-1}$. Changes in temperature and small variations in the chemical composition result only in fairly small changes of the interfacial tension. ${ }^{59-61}$

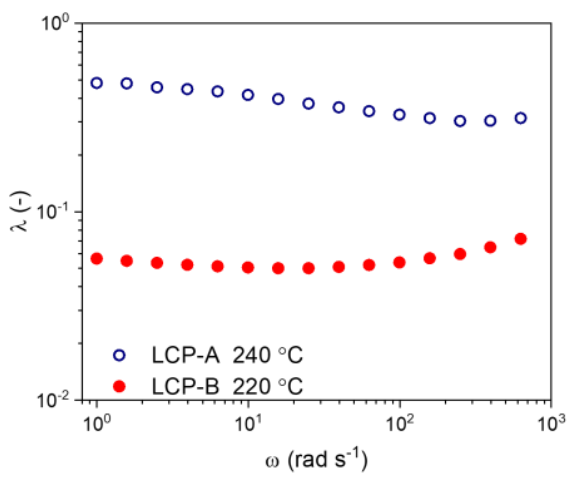

Figure 3.5 Viscosity ratio ( $\lambda$ ) vs frequency ( $\omega$ ) as calculated for both LCP/PLLA blends at the respective processing temperatures.

The previous estimations suggest that dispersion of the LCP occurs readily in a shear flow field as particles subsequently stretch and break up. However, the flow field applied in extrusion is rather complex as it comprises a combination of elongational and shear flow fields that vary along the position within the extruder. As a result, the variation in the flow field with the position of the fluid element in the extruder hinders the formation of stable LCP filaments and promotes breakup instead. As extrusion time increases, the particle size decreases, until a dynamic equilibrium is achieved where a number of distinct types of LCP droplets are expected: 1) Large spherical droplets; these are the result of droplets that have not been broken up or have been formed due to coalescence events and are expected to deform and break up in the future. 2) Deformed large droplets; due to their initial large diameter, the flow field is in the process of stretching them into filaments that are stable or later break up into two droplets. 3) 
Fibrils which originate from larger droplets; they have not been stretched so far as to become instable and break up. 4) Small, spherical droplets which are the product of breakup events; These droplets have a low value of $x^{*}$ due to their small size and are not deformed further by the imposed flow fields.

Large LCP fibrils are expected to be rare in the barrel of the extruder, as the varying flow field is likely to cause instability. Therefore, the droplet morphology is expected to consist mostly of types 1,2 and 4. However, as the molten blend exits the extruder, the barrel tapers into a narrower channel, creating a zone with a dominant extensional flow profile. In this zone, droplets with high aspect ratios (type 3) are expected to be the dominant morphology.

When relating this to the LCP-B/PLLA blends, we expect a decreased average droplet diameter compared to the LCP-A/PLLA blends. Recall, the blends containing LCP-B are processed at $220^{\circ} \mathrm{C}$, leading to a higher $\eta_{\text {matrix }}$ and $\varkappa$, and thus more effective droplet deformation and breakup. Additionally, the viscosity ratio for these blends is lower compared to that of the LCP-B/PLLA blends, reducing the timescale for breakup. As such, the dynamic equilibrium between breakup and coalescence will be pushed into the direction of smaller particles. Regardless of which LCP-component was used in the blend, the overall droplet size will increase with the volume fraction of LCP, as a higher number droplets of a given size is required at a higher volume fraction. This leads to a higher collision frequency, pushing the dynamic equilibrium towards larger droplets.

To verify the accuracy of these predictions, the extrudates of both LCP-A/PLLA and LCP-B/PLLA blends containing $10 \mathrm{wt} \%$ and $30 \mathrm{wt} \%$ LCP were analysed in polarized optical microscopy (Figure 3.6). In order to study the LCP morphology, the PLLA was dissolved in a mixture of acetone and dichloromethane (1:3 by volume). Overall, the observed LCP particle morphologies agree rather well with expectations; The average particle size is smaller for blends containing LCP-B and the particle size increases with increasing LCP content for both blends. Furthermore, stretched LCP fibrils are detected combined with two populations of spherical LCP particles with a clear separation in size. In the $10 \mathrm{wt} \%$ LCP-B/PLLA blend, elongated structures are largely absent. The reason for that might be two-fold: the low average diameter in this blend, in combination with quicker expected timescale for breakup, has led to breakup on timescales shorter than that of the quenching; or these structures are destroyed largely as the matrix is dissolved. Additionally, the shape of most of the fibrillary structures in the other samples is not truly fibrillary, instead the fibrils contain thick, droplet like segments, separated by thinner, seemingly stretched segments. Their appearance suggests that the filaments were not stable and in the process of breaking up. This can be attributed to the lack of stress as the strand exits the die in combination with the 
timescale of quenching. Nevertheless, the observed LCP particle morphologies are in excellent agreement with the morphology expected from theory.
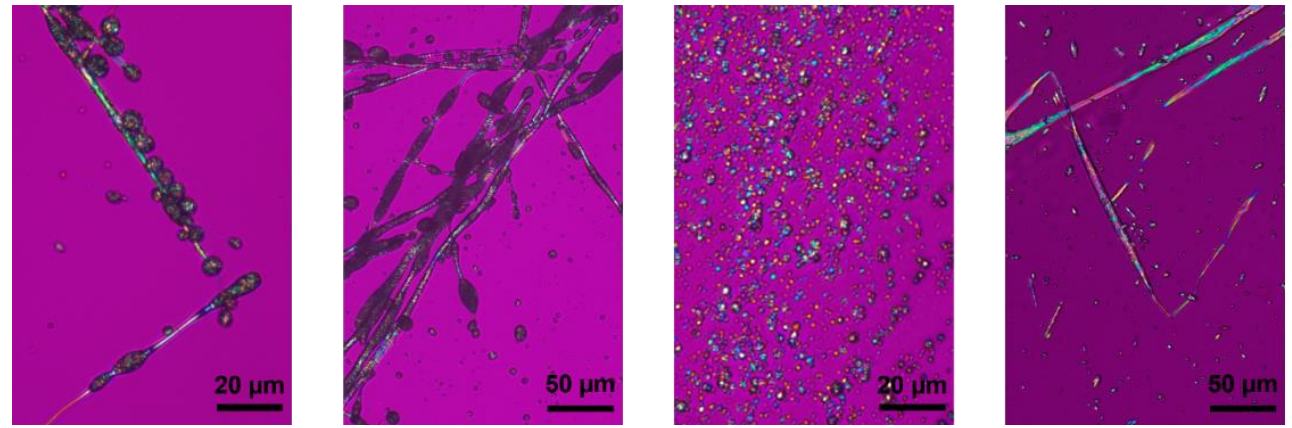

Figure 3.6 LCP particle morphology of blend extrudates. From left to right: $10 w t \%$ LCP-A in PLLA, 30 $w t \%$ LCP-A in PLLA, $10 w t \%$ LCP-B in PLLA, and $30 w t \%$ LCP-B in PLLA. Note, the PLLA matrix was dissolved in a 1:3 mixture of acetone/dichloromethane prior to capturing the LCP particle image, causing the formation of agglomerates of individual particles.

\section{LCP particle and interchain relaxation during isothermal conditions}

Prior to injection molding of the sample, the molten blend is transferred into a preheated barrel, where it is maintained under quiescent and isothermal conditions at a high temperature for some time (in the order of $10 \mathrm{~s}$ to $1 \mathrm{~min}$ ). During this isotherm, the LCP droplets experience no stresses and deformed LCP particles will either contract or break up into multiple droplets. The timescale for breakup will be shorter in LCPB/PLLA blends resulting from their decreased viscosity ratio compared to LCPA/PLLA blends. Additionally, combined with the intrinsic relaxation of LCP upon the cessation of flow, the decreasing average aspect ratio of the particles leads to a rapid decrease in the interchain orientation of the LCP within the particles. As a result, after the isotherm, an LCP particle morphology consisting of only spherical droplets of various sizes is expected. Indeed, this seems to be the scenario, as is visible from the optical micrographs in Figure 3.7. The presence of only spherical LCP particles confirms full relaxation of oriented LCP particles during the residence in the barrel. Furthermore, as observed earlier, two populations of spherical droplets with different diameters are present; and the expected differences in particle morphology resulting from the LCP concentration remain clearly visible. The minimum particle size was considerably larger than the estimated theoretical minimum value, as is expected for blends of viscoelastic materials ${ }^{56}$. A more elaborate analysis of the particle morphology is available in the Supporting Information.

In addition to loss of interchain orientation of LCP melts due to changes in blend morphology, the interchain orientation in LCPs is known to decrease upon the cessation 
of flow. In order to estimate the time-scale of this process, the intrinsic relaxation behavior of the LCPs has been evaluated. In Chapter 1, under conditions where the LCP viscosity is constant as a function of shear rate, we have shown that the relaxation of the interchain orientation scales linearly with the applied shear rate and that nematic texture relaxes via a two-step mechanism ${ }^{62}$. Initially a fast contraction of the domain texture occurs, followed by a slower coalescence of the liquid crystalline domains. As a result, LCP melts subjected to higher shear stress display enhanced interchain relaxation rates ${ }^{62,63}$.
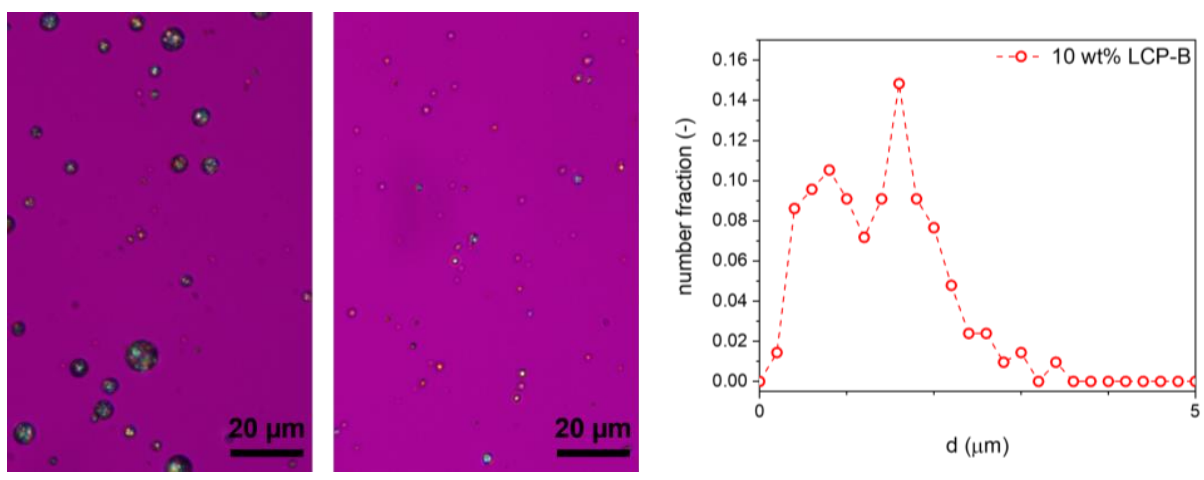

Figure 3.7 LCP particle morphology of extruded strands after heating to the processing temperature for 1 minute. From left to right: $10 w t \%$ LCP-A in PLLA, $10 w t \%$ LCP-B in PLLA, particle size distribution of $10 w t \%$ LCP-B in PLLA. Note, the PLLA matrix was dissolved in a 1:3 mixture of acetone/ dichloromethane prior to capturing the LCP particle image.

The shear response of LCPs typically shows a minimum in viscosity at low strain, followed by a maximum, and at sufficiently large strains, a plateau is reached. These phenomena are correlated to the development of interchain orientation and the domain texture of the LCP, and can be used as an indicator for the relaxation behavior ${ }^{64}$. In order to confirm that the experiments conducted on both LCPs are performed in the regime where the LCP plateau viscosity is constant as a function of shear rate $e^{65}$, the LCPs were subjected to a constant shear stress until a plateau value was reached. Figure 3.8a shows the plateau shear rate as a function of the applied shear stress. The observed shear rates seem to follow a slope of 1.15 , marked by the dotted lines, which is close to the expected slope of 1 . The plateau viscosity as a function shear rate is shown in Figure 3.8b. The viscosity of LCP-A is roughly a factor 10 larger compared to that of LCP-B over the measured range of shear rates. Additionally, the plateau viscosity is indeed almost constant with respect to the shear rate, indicating that both LCPs are indeed in the expected regime. The only exception is the measurement of LCP-A at the lowest applied shear stress, which has a considerably higher viscosity. 
a

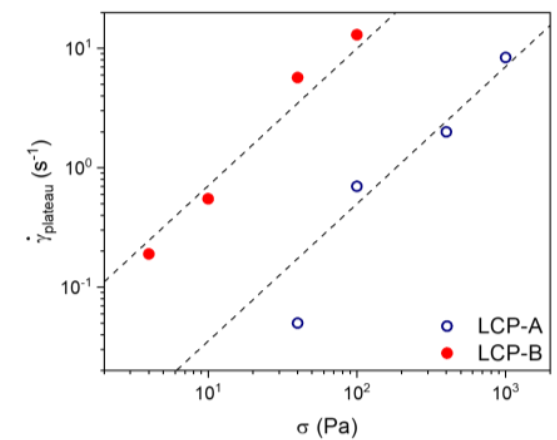

b

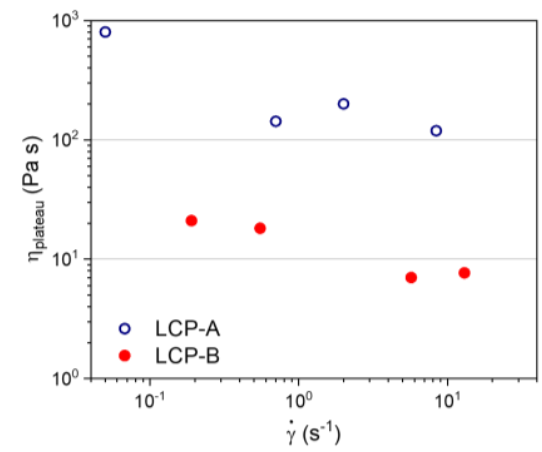

Figure 3.8 (a) Steady state shear rate vs applied shear stress for both LCPs. (b) Steady state viscosity vs shear rate for both LCPs. Data was collected at the respective extrusion temperatures of the LCP/PLLA blends.

To identify the rate of the domain coalescence process, which is linked to decreasing interchain orientation, the samples were sheared at a constant shear stress until the equilibrium state was reached (at least 400 s.u.). Next, the deformation was stopped and the molten LCP was subjected to a relaxation time $\tau$, varying from 1 to $1000 \mathrm{~s}$. The evolution of the viscosity as a function of shear strain after the reapplication of shear is shown in Figure 3.9.
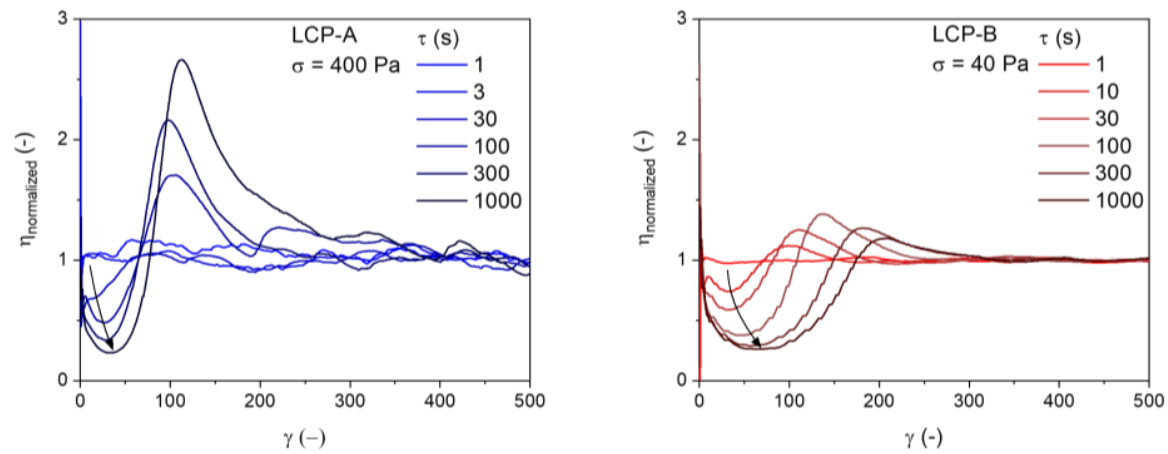

Figure 3.9 Transient behavior of both LCPs following different relaxation periods. Data was collected at the respective extrusion temperatures of the LCP/PLLA blends.

In both LCPs, for short relaxation times $(\tau=1 \mathrm{~s})$, the viscosity increases at very low strains as the shear stress is reapplied, and reaches a constant value almost immediately. The minimum and maxima, characteristic for the evolution of the domain texture and interchain orientation, are completely absent; indicating that on this timescale relaxation has a negligible influence on the polydomain texture of the LCPs and domain coalescence has not progressed significantly. As the relaxation time increases, the characteristic minimum and maximum become more significant, indicating a further progression of the textural relaxation. Since the evolution of the orientation parameter 
and the texture are closely related, these experiments indirectly give information with respect to interchain orientation: a lack of textural relaxation indicates that interchain orientation is maintained whereas strong textural relaxation indicates loss of interchain orientation.

a

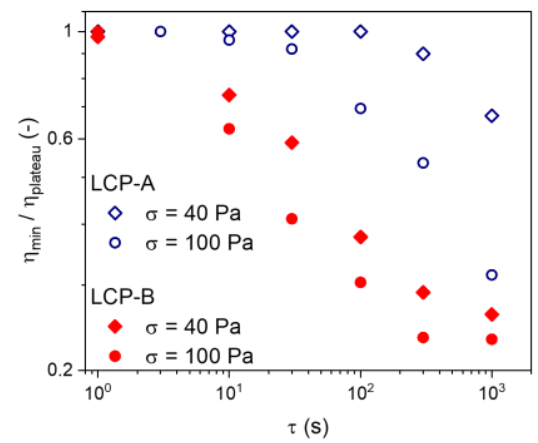

b

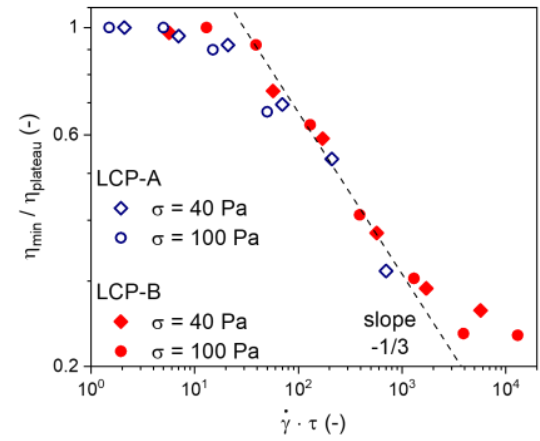

Figure 3.10 (a) Textural relaxation of the LCPs as a function of relaxation time. (b) Normalized with the steady state shear rate. Data was collected at the respective extrusion temperatures of the LCP/PLLA blends.

Figure 3.10a shows the evolution of the textural relaxation after different stresses have been applied to both LCP-B and LCP-A. The ratio of the viscosity of the minimum with respect to the plateau was used to characterize the textural relaxation. Lower values correspond to a texture closer to the quiescent texture, hence a further progression of the textural relaxation and thus a decrease in interchain orientation. Overall, we observe that, at similar shear stresses, the LCP texture relaxes roughly 10 times faster in LCP-B compared to LCP-A. This large difference in relaxation time therefore seems to be related to the lower viscosity and the more flexible backbone of the aromatic-aliphatic LCP-B. Furthermore, for both LCPs, we observe that the textural relaxation slows down and levels off at long timescales, which would correspond to the LCPs approaching the quiescent texture. As expected, the application of higher stresses result in a faster relaxation, originating from the increase in applied shear rate. Figure $3.10 \mathrm{~b}$ shows the same relaxation experiments, but with the relaxation time normalized for the applied shear rate, effectively compensating for differences in viscosity. It is noteworthy that the normalized data from the different experiments falls on the same curve. The decrease in the ratio consistently followed a slope of $-1 / 3$ for both LCPs over the range of applied shear stresses. Overall, this data confirms that the LCP texture and interchain relaxation is significantly enhanced after the application of increasing shear stresses and with decreasing LCP viscosity. Note the magnitude of the applied shear stresses in these experiments needs to be considered: it is considerably lower than what one would expect in the actual processing of blends and therefore extrapolation is required to 
estimate the effect relaxation during the quiescent period in the barrel. A 10- to 100fold increase in shear stress would shift the timescales for textural relaxation well within the 10-second range, the residence time of the molten blend in the barrel. In combination with the breakup and retraction of fibrils due to a lack of stabilizing stresses, no residual interchain orientation of the LCPs is expected in any of the blends at this stage in the processing.

\section{Injection molding}

Subsequently to its residence in the heated barrel, the molten blend is pushed out of the barrel into a cold, dogbone shaped mold. In this processing step the material is subjected to high cooling rates in combination with a complex flow field and high deformation rates. As a pressure is applied, the molten blend is forced out of the barrel, through a tapered die, into the mold. Due to the onset of deformation, the collision frequency increases tremendously and coalescence can occur again; droplets are effectively deformed into fibrils as the blend is forced out of the barrel. As the mold is dogbone shaped, the resulting flow field is complex and dependent on the actual position of the fluid element. Nevertheless, it is generally accepted that injection molded polymer next to the wall of the mold is unable to flow and is subject to high shear rates and shear stress. In contrast, when moving further away from the wall of the mold, the polymers are subject to lowered shear stresses and are able to flow. Typically, a shear layer is formed close to the mold wall in injection molding as a result of both the high shear rate and cooling rate. Due to this rapid cooling, the PLLA chains residing close to the wall orient along the flow direction and are immediately quenched to temperatures below the $T_{\mathrm{g}}$, effectively preventing their crystallization. A similar deformation scenario is expected for the dispersed LCP droplets, where the shear rate close to the wall results in deformation and rapid molecular orientation during the cooling process. However, due to the flow limitations at the wall, the LCP morphology close to the mold wall is expected to be ribbon like.

Towards the core of the bar, the molten blend experiences a decreasing cooling rate. The blend that is not in contact with the mold walls will therefore change in viscosity during this cooling process, effectively resulting in an increase in viscosity ratio. Figure 3.11a shows the expected change in viscosity ratio, as is evaluated using plate-plate rheology. In general, we observe that the viscosity ratio gradually increases from approximately 0.5 to 3 in LCP-A/PLLA blends as the temperature range drops from the processing temperature to $160{ }^{\circ} \mathrm{C}$. In contrast, LCP-B, crystallizes rapidly as the material is cooled to temperature below $200{ }^{\circ} \mathrm{C}$ effectively resulting in an increase in viscosity ratio from 0.06 to values close to 100 as the temperature decreases from the 
processing temperature to $160{ }^{\circ} \mathrm{C}$.

a

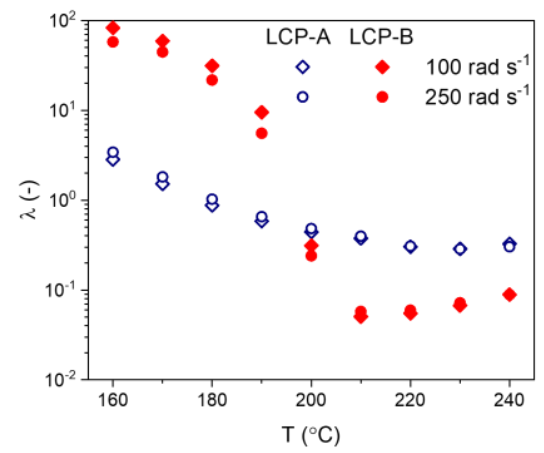

b

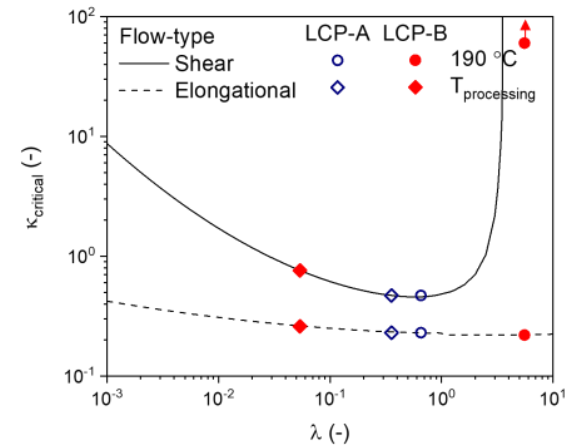

Figure 3.11 (a) Evolution of the viscosity ratio for the two different LCP/PLLA blends with temperature. (b) The effect of cooling on the critical capillary number for the LCP/PLLA blends.

The implications of the changes in the viscosity ratio for $x_{\text {critical }}$ are depicted in Figure 3.11b. In the case of LCP-A/PLLA blends, $x_{\text {critical }}$ remains between 0.2 and 0.5 , depending on the flow field (at least during cooling to $160{ }^{\circ} \mathrm{C}$ ), correspondingly causing values for $x^{*}$ to remain high. Recall, under these conditions, particle deformation into stable filaments is favored; the extended filaments are stabilized quite well by shear stresses during cooling. In contrast, in the case of LCP-B, the viscosity ratio increases dramatically over the temperature range between $200{ }^{\circ} \mathrm{C}$ and $160{ }^{\circ} \mathrm{C}$. This causes $x_{\text {critical }}$ to rise drastically and $x^{*}$ to drop significantly. Consequentially, LCP droplets cannot be deformed effectively by a shear-type flow field anymore, resulting in a significantly decreased droplet deformation and thus a lowered degree of interchain orientation of the LCP-B particles.

Combining the LCP particle morphology prior to injection molding (Figure 3.7) with the difference in deformation behavior of the LCP particles based on the changes in $x_{\text {critical }}$ during cooling (Figure 3.11b) allows for a rough prediction of the final LCP morphology after injection molding. LCP-A/PLLA blends are expected to yield relatively thick LCP filaments with very high aspect ratios, owing from the rather large LCP droplet size prior to injection molding. In contrast, the LCP-B/PLLA blends are expected to contain significantly thinner LCP fibrils with low aspect ratios, owing to their finer starting morphology and the lower stability of fibrils due to the rapid increase in viscosity ratio upon crystallization. Furthermore, in both blends, the small droplets that were initially present are, due to the tendency of small droplet to rotate rather than deform in shear, likely to be unaffected by the imposed flow fields. For this reason, in addition to oriented LCP particles a distribution of small LCP droplets can be expected. 
The LCP particle morphology and the interchain orientation of the LCP phase after injection molding were analyzed using SEM and WAXD (Figure 3.12, blends containing $30 \mathrm{wt} \% \mathrm{LCP})$. The analysis of the blends containing 10\% LCP are provided in Figure S3.4 in the Supporting Information. Indeed, as expected, both samples contain clear shear layers (Figure 3.12, left), although the expected ribbon-like particle morphology is only clearly observed for LCP-A/PLLA composites. Towards the core of the sample, we observe that LCP-A indeed is present as the expected thick elongated fibrils. Indeed, the LCP-B particles do not seem to be as elongated as is the case for LCP-A, but instead persist as short fibrils or nodules.

Skin
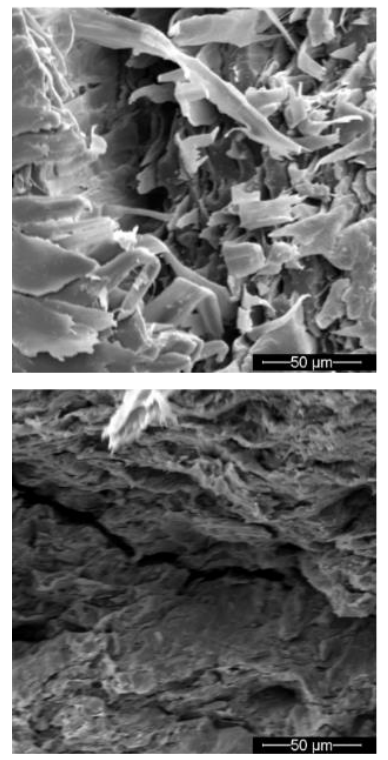

Core
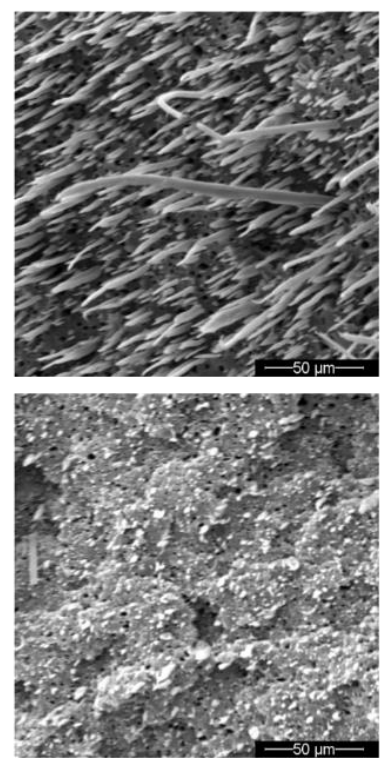

WAXD
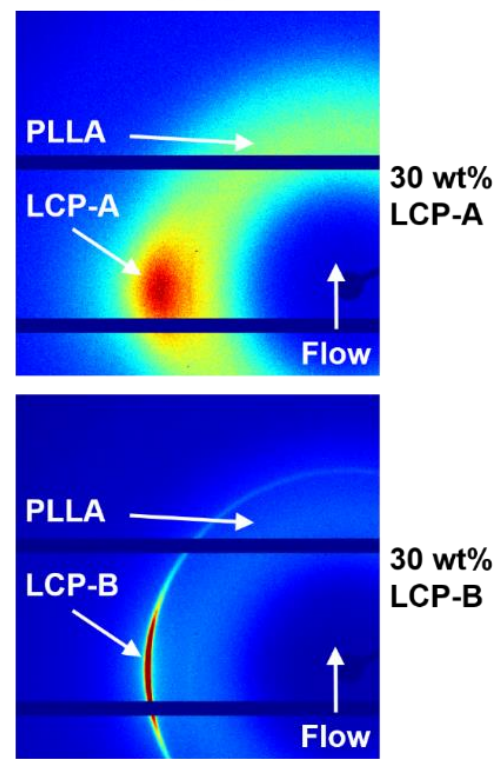

$30 \mathrm{wt} \%$ LCP-B

Figure 3.12 Microstructure of injection molded samples: SEM-images of sample skin (left), SEM-images of sample core (middle), diffraction patterns (right).

The 2D-WAXD diffraction patterns of the produced bars show an amorphous halo in the lower q-range, corresponding to the presence of amorphous PLLA throughout the sample. The LCP components are detected at slightly higher q-range and are visibly oriented, indicated by the presence of arcs of the interchain diffraction signals. Interestingly, the diffraction of LCP-B show an isotropic peak superimposed on the arc, suggesting an inhomogeneous degree of orientation throughout the sample. Such diffraction behavior is related to the spatial variation in LCP morphology in the sample; LCP-B residing in the shear layer will exhibit high interchain orientation, whereas the small nodules in the core of the sample are expected to give rise to isotropic scattering. Such behavior is not observed in LCP-A/PLLA samples, confirming that indeed the 
LCP-A has a high interchain orientation, both in the shear layer and in the core of the bar.

\section{Melt-Drawing}

To prepare melt-drawn PLLA/LCP blends, the LCP and PLLA pellets are fed into the twin-screw extruder and the same extrusion conditions were applied as for the injection molding process. Contrary to the injection molding route, where the blends are extruded into a barrel to remain quiescent for a time after extrusion, the molten blend is pushed through a slit die, and the tape is simultaneously cooled by air and stretched by a rotating roll. In other words, the blend is continuously subjected to an extensional flow field after it leaves the extruder, until it solidifies due to air-cooling. Under such conditions, extended LCP fibrils are stabilized in all blend compositions during the entire drawing process, as is reported by Song and Isayev ${ }^{31}$. Indeed, such behavior is expected for both LCP-A and LCP-B based blends, as the sudden increase in viscosity ratio upon the crystallization of LCP- B does not lead to a drastic increase in $x_{\text {critical }}$ in an extensional flow field (Figure 3.11b). Though the presence of elongated fibrils is expected in both blends, the possibility of fibril breakup can not be excluded; Due to the continuous drawing, the fibrils are continuously decreasing in diameter and therefore subject to lower stresses, possibly causing instability and breakup. Breakup in this fashion would result in the generation of a large number of small droplets ${ }^{54}$. To recall, such small droplets with a diameter in the range of a few micrometer, are not as effectively deformed by the flow field and might therefore persist. Nevertheless, such droplets might disappear due to coalescence with other droplets or fibrils in the deforming melt.

In addition, the high cooling rate that the tapes encounter stabilizes the fibrillary LCP morphology and allows its features to be frozen in a highly stretched state before relaxation can occur. Figure 3.13 shows the morphology of the LCP fibrils in the obtained tapes. Indeed, as expected, the dominant species are fibrils with a high aspect ratio $(>100)$, though a small amount of droplets is detected as well. The homogeneous coloring of the fibrils in the optical micrographs indicates that the chains inside the fibrils are all oriented in the same direction, thus suggests a high degree of interchain orientation. Indeed, the high degree of interchain orientation is also observed from WAXD, as is shown in Figures S3.8 of the Supporting Information. Again, because of the starting morphology after extrusion (Figure 3.7), LCP-B tapes to contain thinner LCP fibrils compared to LCP-A/PLLA tapes. A similar trend is observed between blends having $30 \% \mathrm{LCP}$ and $10 \% \mathrm{LCP}$, again resulting from the difference in the LCP particle morphology generated in the extrusion step. Overall, this data confirms that the 
limitations in LCP particle orientation by the shear components in the flow fields present in injection molding is readily overcome through the usage of only an extensional flow as is the case in melt-drawing.
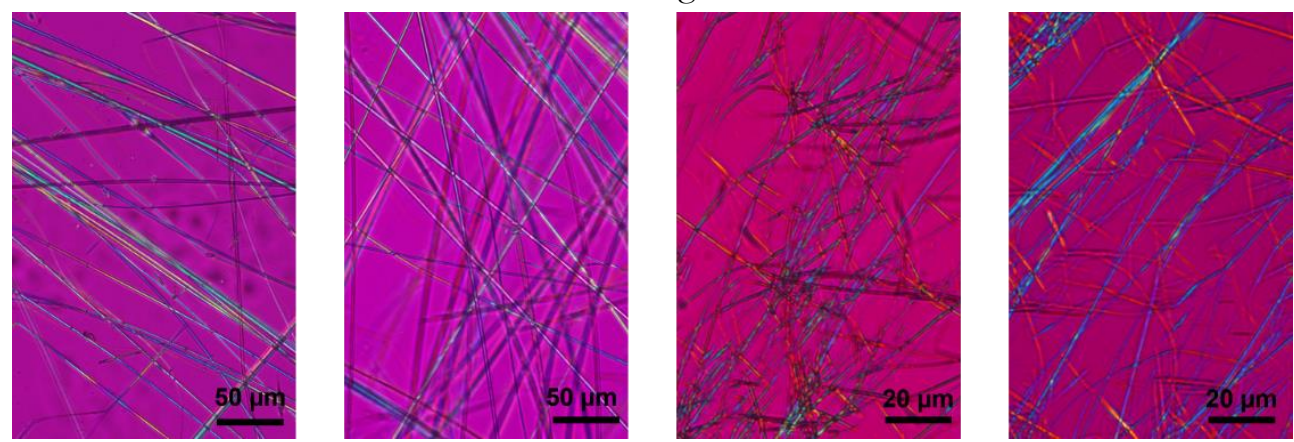

Figure 3.13 LCP fibril in melt-drawn tapes. From left to right: $10 w t \%$ LCP-A in PLLA, $30 w t \%$ LCPA in PLLA, $10 w t \%$ LCP-B in PLLA, and 30 wt\% LCP-B in PLLA. Note, the PLLA matrix was dissolved in a 1:3 mixture of acetone/dichloromethane prior to capturing the LCP particle image.

\section{Relation between particle morphology, interchain orientation and mechanical performance}

In order to confirm whether effective reinforcement in the LCP/PLLA composites was achieved, the mechanical properties of the produced tapes and dogbones were measured in extension. The results are summarized in Table 3.1 whereas the characteristic stress-strain curves are depicted in Figure 3.14. Additionally, the orientation parameter $\mathrm{S}$, as calculated from WAXD is provided in Table 3.1 as well. In general, we observe that the tensile modulus of the tapes is significantly higher than for the amorphous PLLA tapes. Additionally, the tensile modulus of the tapes containing LCP is higher than that of the dogbones with the same composition. This effect is undoubtedly caused by the higher aspect ratio of the LCP particles combined with their increased interchain orientation, as observed during the morphological analysis. Notably, the increase in stiffness and stress at break for LCP-B/PLLA tapes is small compared to the LCP-A/PLLA tapes, although in both cases the morphology was suitable for effective reinforcement. This stems from the aromatic-aliphatic nature of the LCP-B, which is known to result in both a lowered strength and stiffness ${ }^{25,66}$. Interestingly, the strain at break is greatly increased for tapes with $30 \mathrm{wt} \%$ LCP-B, in addition to an increased modulus and a higher yield stress, effectively toughening the PLLA phase. Such behavior has been observed earlier for a similar LCP dispersion in poly( $\omega$-pentadecalactone), and is expected to originate from surface effects resulting from the very fine LCP morphology ${ }^{19}$. In general, LCP-A seems to be an efficient reinforcing filler for injection molding, as the modulus and the stress at break increase 
greatly with increasing LCP content. This is not the case for dogbones containing LCP$\mathrm{B}$; although the modulus and stress at break are enhanced for the $10 \mathrm{wt} \%$ samples, higher LCP content leads to a decrease in mechanical properties. This is in line with observations based on SEM, where we observed that LCP particles maintain mostly a nodular morphology. The decreasing aspect ratio with increasing LCP-B content appears detrimental for effective reinforcement. Interestingly, injection molding the pure LCP-B yields tensile bars with a rather poor tensile modulus for thermotropic LCPs $(1.5 \mathrm{GPa})$. This behavior is resulting from the high shear stress applied during injection, which accelerate both the orientation and the relaxation of the interchain orientation. Indeed, due to the low viscosity of the LCP-B, relaxation of the oriented nematic domains is readily achieved during the cooling in injection molding, effectively yielding tensile bars with low interchain orientation. Overall, from this data we can conclude efficient LCP reinforcement of the PLA matrix required the presence of both highly elongated LCP firbils and a high interchain orientation inside the LCP component.

Table 3.1 Mechanical properties and orientation parameter of produced blends.

\begin{tabular}{|c|c|c|c|c|c|}
\hline & & $\begin{array}{l}\text { LCP content } \\
\left(w t^{\circ} \%\right)\end{array}$ & $\begin{array}{l}\text { E-modulus } \\
(\mathrm{GPa})\end{array}$ & $\sigma_{\max }(\mathrm{MPa})$ & S (-) \\
\hline \multirow{5}{*}{ tape } & PLLA & 0 & $3.1 \pm 0.26$ & $36 \pm 3.3$ & 0 \\
\hline & \multirow{2}{*}{ LCP-A } & 10 & $6.1 \pm 0.49$ & $58 \pm 4.3$ & $>0.8$ \\
\hline & & 30 & $13.3 \pm 0.75$ & $145 \pm 10$ & $>0.8$ \\
\hline & \multirow{2}{*}{ LCP-B } & 10 & $4.8 \pm 0.75$ & $61 \pm 4.8$ & $>0.8$ \\
\hline & & 30 & $5.5 \pm 0.59$ & $59 \pm 8.8$ & $>0.8$ \\
\hline \multirow{9}{*}{$\begin{array}{c}\text { Injection } \\
\text { molded } \\
\text { bar }\end{array}$} & PLLA & 0 & $3.6 \pm 0.22$ & $67 \pm 5.1$ & 0 \\
\hline & \multirow{4}{*}{ LCP-A } & 10 & $5.4 \pm 0.17$ & $100 \pm 1.8$ & 0.65 \\
\hline & & 20 & $6.5 \pm 0.30$ & $127 \pm 3.2$ & 0.61 \\
\hline & & 30 & $8.0 \pm 0.30$ & $138 \pm 2.9$ & 0.65 \\
\hline & & 100 & $17.0 \pm 0.65$ & $251 \pm 7.9$ & 0.75 \\
\hline & \multirow{4}{*}{ LCP-B } & 10 & $4.2 \pm 0.03$ & $77 \pm 3.2$ & 0.75 \\
\hline & & 20 & $3.7 \pm 0.03$ & $73 \pm 4.1$ & 0.62 \\
\hline & & 30 & $3.9 \pm 0.12$ & $68 \pm 0.7$ & 0.64 \\
\hline & & 100 & $1.5 \pm 0.21$ & $35 \pm 4.1$ & 0.49 \\
\hline
\end{tabular}




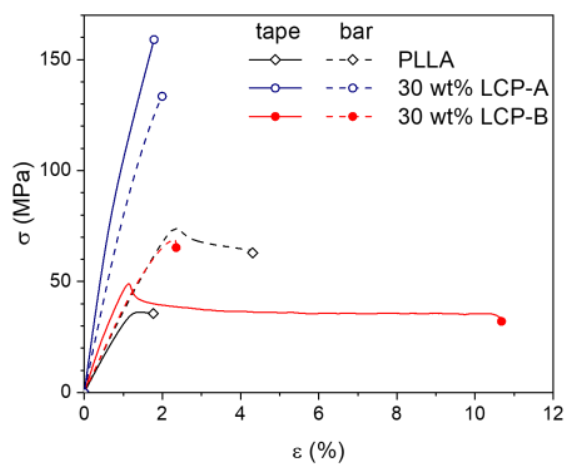

Figure 3.14 Characteristic stress-strain curves of produced tapes and injection molded samples.

\subsection{Conclusions}

In this study, blends of two different thermotropic LCPs in PLLA are produced and the effect of the chosen processing routes is evaluated in terms of LCP morphology, LCP orientation and composite mechanical properties. Elongated LCP fibrils with a high degree of interchain orientation result in effective reinforcement of the PLLA matrix, and are readily generated when the LCP/PLLA blends are melt-drawn into tapes. In contrast, the morphology and performance of the LCP/PLLA blends proved significantly different when subjected to injection molding. From the findings in this work, the following design rules can be gathered for the development of LCP materials for injection molding of thermoplastic LCP/PLLA blends:

- In injection molding, the morphology of the extrudate and the thermal dependence of the flow behavior are found to be of importance. A very fine particle distribution can prove detrimental in achieving reinforcement: The shear dominated flow field can be ineffective to deform small spherical droplets, therefore relying on coalescence in the initial stages of the process to allow the formation of fibrils. To this end, the matrix viscosity and the viscosity ratio are identified as key parameters in the extrusion process as they govern deformation and breakup of droplets in the complex flow field, and influencing both the resultant morphology and the particle size distribution. Therefore, the viscosity of the LCP component should be matched with the viscosity of the thermoplastic matrix (i.e. $\lambda \leq 1$ ) to ensure the generation of LCP particles that can efficiently be deformed during injection molding.

- Crystallization of the dispersed LCP is shown to hinder reinforcement during the injection molding process, as it induces a sudden increase in viscosity ratio, 
destabilizing fibrils and hindering deformation. Therefore, these findings suggest that the thermal behavior of the LCP should be matched to the thermal behavior of the matrix under processing conditions: Given the fact that PLLA does not crystallize during the injection molding process, the use of an amorphous LCP is preferred over the use of a semi-crystalline LCP that crystallizes during the injection molding process. More generally, it is concluded that a rapid increase in viscosity ratio during cooling, hampers the formation of a fibrillary morphology in process that involve mostly shear flow.

- In processes involving predominantly elongational flow, such as melt drawing, the viscosity ratio, and its development with temperature is of less significance. An elongational flow field is more effective in deforming and stabilizing LCP fibrils, regardless of the viscosity ratio, and tapes with a highly oriented fibrillary LCP phase can readily be obtained.

- The rheological findings in this work demonstrate that the LCP viscosity is of crucial importance for the interchain orientation and relaxation processes. The relaxation time of the LCP material is strongly governed by the contraction of the oriented nematic LCP domains, which in turn enhanced by the application of increasing shear rates or decreasing LCP viscosity. Therefore, to maintain the LCP orientation during injection molding, the usage of a high viscosity LCP component is desired. As is observed for the injection molding of pure LCP-B, too low LCP viscosity results in a rapid loss of the induced interchain orientation already during the injection molding process. Interestingly, this process does not seem to depend on the chemical structure of the LCP, it only depends on the LCP viscosity (Figure 3.10a).

As noted in the points before, the chemical composition of the LCP components does not directly affect the morphological development in the LCP/PLLA blends during processing, although it is an important factor with respect to the viscosity and thermal behavior of the LCP and interfacial tension of the blend. However, for a maximum reinforcement of the PLLA matrix, it is desired to take the intrinsic performance of the LCP material into account. In the ideal scenario, the thermoplastic LCP/PLLA composite will display performance according to the rule of mixtures, where the contribution of the LCP component is governed by its loading, its intrinsic mechanical performance, and its interchain orientation. To this end, the usage of fully aromatic thermotropic polyesters is desired as the presence of aliphatic spacers deteriorates both the maximum tensile modulus and tensile strength of the LCP component. 


\subsection{References}

(1) Song, Y. S.; Youn, J. R.; Gutowski, T. G. Life Cycle Energy Analysis of Fiber-Reinforced Composites. Compos. Part A Appl. Sci. Manuf. 2009, 40 (8), 1257-1265. https://doi.org/10.1016/j.compositesa.2009.05.020.

(2) Pimenta, S.; Pinho, S. T. Recycling Carbon Fibre Reinforced Polymers for Structural Applications: Technology Review and Market Outlook. Waste Manag. 2011, 31 (2), 378 392. https://doi.org/10.1016/j.wasman.2010.09.019.

(3) Asmatulu, E.; Twomey, J.; Overcash, M. Recycling of Fiber-Reinforced Composites and Direct Structural Composite Recycling Concept. J. Compos. Mater. 2014, 48 (5), 593-608. https://doi.org/10.1177/0021998313476325.

(4) Bassett, B. R.; Yee, A. F. A Method of Forming Composite Structures Using in Situ Formed Liquid Crystal Polymer Fibers in a Thermoplastic Matrix. Polym. Compos. 1990, 11 (1), 10-18. https://doi.org/10.1002/pc.750110103.

(5) Wang, H.; Lee, K. W.; Chung, T. S.; Jaffe, M. Rheology, Morphology and Properties of LCP/Nylon 66 Composite Fibers. Polym. Compos. 2000, 21 (1), 114-123. https://doi.org/10.1002/pc.10170.

(6) Hassan, A.; Yahya, R.; Yahaya, A. H.; Tahir, A. R. M.; Hornsby, P. R. Tensile, Impact and Fiber Length Properties of Injection-Molded Short and Long Glass FiberReinforced Polyamide 6,6 Composites. J. Reinf. Plast. Compos. 2004, 23 (9), 969-986. https://doi.org/10.1177/0731684404033960.

(7) Fu, S. Y.; Lauke, B.; Mäder, E.; Yue, C. Y.; Hu, X. Tensile Properties of Short-GlassFiber- and Short-Carbon-Fiber-Reinforced Polypropylene Composites. Compos. Part A Appl. Sci. Manuf. 2000, 31 (10), 1117-1125. https://doi.org/10.1016/S1359835X(00)00068-3.

(8) Chand, S. Carbon Fibers for Composites. J. Mater. Sci. 2000, 35 (6), 1303-1313. https://doi.org/10.1023/A:1004780301489.

(9) Crist, B. The Ultimate Strength and Stiffness of Polymers. Annu. Rev. Mater. Sci. 1995, 25, 295-323.

(10) Kiss, G. In Situ Composites: Blends of Isotropic Polymers and Thermotropic Liquid Crystalline Polymers. Polym. Eng. Sci. 1987, 27 (6), 410-423. https://doi.org/10.1002/pen.760270606.

(11) Blizard, K. G.; Baird, D. G. The Morphology and Rheology of Polymer Blends Containing a Liquid Crystalline Copolyester. Polym. Eng. Sci. 1987, 27 (9), 653-662. https://doi.org/10.1002/pen.760270909.

(12) Blizard, K. G.; Federici, C.; Federico, O.; Chapoy, L. L.; Caduti, V. The Morphology of Extruded Blends Containing a Thermotropic Liquid Crystalline Polymer. Polym. Eng. Sci. 1990, 30 (22), 1442-1453. https://doi.org/10.1002/pen.760302205.

(13) Silverstein, M. S.; Hiltner, A.; Baer, E. Hierarchical Structure in LCP/PET Blends. J. Appl. Polym. Sci. 1991, 43 (1), 157-173. https://doi.org/10.1002/app.1991.070430113.

(14) Economy, J. Aromatic Polyesters of P-Hydroxybenoic Acid. Mol. Cryst. Liq. Cryst. 1989, 169, 1-22. https://doi.org/10.1080/00268948908062731.

(15) Demus, D.; Goodby, J.; Gray, G. W.; Spiess, H. W.; Vill, V. Handbook of Liquid Crystals; Wiley-VCH, 1998. https://doi.org/10.1002/9783527620593.

(16) Collyer, A. A. Thermotropic Liquid Crystal Polymers for Engineering Applications Applications. Mater. Sci. Technol. 1989, 5, 309-322. https://doi.org/10.1179/mst.1989.5.4.309.

(17) Yang, Q.; Hirata, M.; Hsu, Y. I.; Lu, D.; Kimura, Y. Improved Thermal and Mechanical Properties of Poly(Butylene Succinate) by Polymer Blending with a Thermotropic 
Controlling processing, morphology and mechanical performance in blends of polylactide and thermotropic polyesters.

Liquid Crystalline Polyester. J. Appl. Polym. Sci. 2014, 131 (4), 1-8. https://doi.org/10.1002/app.39952.

(18) Yang, Q.; Hirata, M.; Lu, D.; Nakajima, H.; Kimura, Y. Highly Efficient Reinforcement of Poly-l-Lactide Materials by Polymer Blending of a Thermotropic Liquid Crystalline Polymer. Biomacromolecules 2011, 12 (2), 354-358. https://doi.org/10.1021/bm1011135.

(19) Wilsens, C. H. R. M.; Pepels, M. P. F.; Spoelstra, A. B.; Portale, G.; Auhl, D.; Deshmukh, Y. S.; Harings, J. A. W. Improving Stiffness, Strength, and Toughness of Poly $(\omega-$ Pentadecalactone) Fibers through in Situ Reinforcement with a Vanillic Acid-Based Thermotropic Liquid Crystalline Polyester. Macromolecules 2016, 49 (6), 2228-2237. https://doi.org/10.1021/acs.macromol.5b02419.

(20) Auras, R. Poly(Lactic Acid) Synthesis, Structures, Properties, Processing, and Application; Auras, R., Lim, L., Selke, S., Tsuji, H., Eds.; John Wiley \& Sons, Inc.: Hoboken, 2010. https://doi.org/10.1017/CBO9781107415324.004.

(21) Kricheldorf, H. R.; Wahlen, L.; Stukenbrook, T. Biodegradable Liquid-Crystalline Aromatic Polyesters. Macromol. Symp. 1998, 130, 261-270. https://doi.org/10.1002/masy.19981300123.

(22) Nagata, M. Synthesis and Properties of Copolyesters Based on Hydroquinone, Sebacic Acid and p-Hydroxybenzoic Acid. High Perform. Polym. 2001, 13 (2). https://doi.org/10.1088/0954-0083/13/2/323.

(23) Du, J.; Fang, Y.; Zheng, Y. Synthesis, Characterization and Biodegradation of Biodegradable-Cum-Photoactive Liquid-Crystalline Copolyesters Derived from Ferulic

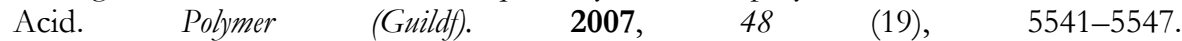
https://doi.org/10.1016/j.polymer.2007.07.040.

(24) Tjong, S. C. Structure, Morphology, Mechanical and Thermal Characteristics of the in Situ Composites Based on Liquid Crystalline Polymers and Thermoplastics. Mater. Sci. Eng. R Reports 2003, 41, 1-60. https://doi.org/10.1016/S0927-796X(03)00035-4.

(25) Chung, T.-S. The Recent Developments of Thermotropic Liquid Crystalline Polymers. Polym. Eng. Sci. 1986, 26 (13), 901-919. https://doi.org/10.1002/pen.760261302.

(26) Picken, S. J.; Sikkema, D. J.; Boerstoel, H.; Dingemans, T. J.; van der Zwaag, S. Liquid Crystal Main-Chain Polymers for High-Performance Fibre Applications. Liq. Cryst. 2011, 38 (11-12), 1591-1605. https://doi.org/10.1080/02678292.2011.624367.

(27) Acierno, D.; Mantia, F. P. La; Polizzotti, G.; Ciferri, A.; Valenti, B. Ultrahigh Modulus Liquid Crystalline Polyesters. Macromolecules 1982, 15 (6), 1455-1460. https://doi.org/10.1021/ma00234a001.

(28) Burghardt, W. R.; Brown, E. F.; Auad, M. L.; Kornfield, J. A. Molecular Orientation of a Commercial Thermotropic Liquid Crystalline Polymer in Simple Shear and Complex Flow. Rheol. Acta 2005, 44 (5), 446-456. https://doi.org/10.1007/s00397-004-0424-1.

(29) Beekmans, F.; Gotsis, A. D.; Norder, B. Transient and Steady-State Rheological Behavior of the Thermotropic Liquid Crystalline Polymer Vectra B950. J. Rheol. (N. Y. N. Y). 1996, 40 (5), 947. https://doi.org/10.1122/1.550788.

(30) Kernick, W. a.; Wagner, N. J. The Role of Liquid-Crystalline Polymer Rheology on the Evolving Morphology of Immiscible Blends Containing Liquid-Crystalline Polymers. J. Rheol. (N. Y. N. Y). 1999, 43 (3), 521. https://doi.org/10.1122/1.551038.

(31) Song, C. H.; Isayev, A. I. LCP Droplet Deformation in Fiber Spinning of SelfReinforced Composites. Polymer (Guildf). 2001, 42 (6), 2611-2619. https://doi.org/10.1016/S0032-3861(00)00555-3.

(32) Heino, M. T.; Hietaoja, P. T.; Vainio, T. P.; Seppälä, J. V. Effect of Viscosity Ratio and Processing Conditions on the Morphology of Blends of Liquid Crystalline Polymer and 
Polypropylene. J. Appl. Polym. Sci. 1994, $51 \quad$ (2), 259-270. https://doi.org/10.1002/app.1994.070510208.

(33) O'Donnell, H. J.; Baird, D. G. The Effect of Injection Molding Conditions on the Mechanical Properties of an in Situ Composite: Polypropylene and a Liquid Crystalline Copolyester Based on Poly(Ethylene Terephthalate) Co Poly(Hydroxybenzoic Acid). Int. Polym. Process. 1996, 11 (3), 257-270. https://doi.org/10.3139/217.960257.

(34) La Mantia, F. P.; Cangialosi, F.; Pedretti, U.; Roggero, A. Extrusion, Spinning and Injection Moulding of Blends of Poly(Ethylene Terephthalate) with Liquid Crystalline Polymers. Eur. Polym. J. 1993, 29 (5), 671-677. https://doi.org/10.1016/00143057(93)90127-2.

(35) Isayev, A. I.; Modic, M. Self- Reinforced Melt Processible Polymer Composites: Extrusion, Compression, and Injection Molding. Polym. Compos. 1987, 8 (3), 158-175. https://doi.org/10.1002/pc.750080305.

(36) Chan, H. S.; Leng, Y.; Gao, F. Processing of PC/LCP in Situ Composites by ClosedLoop Injection Molding. Compos. Sci. Technol. 2002, 62 (6), 757-765. https://doi.org/10.1016/S0266-3538(02)00051-9.

(37) Mitchell, G. R.; Windle, A. H. Orientation in Liquid Crystal Polymers. In Developments in crystalline polymers; Basset, D. C., Ed.; Elsevier Applied Science: London, UK, 1988. https://doi.org/10.1007/978-94-009-1341-7_3.

(38) Einstein, A. Eine Neue Bestimmung Der Molekuldimensionen. Ann. Phys. 1906, 19, 289. https://doi.org/10.1002/andp.19063240204.

(39) Einstein, A. Berichtigung $\mathrm{Zu}$ Meiner Arbeit: "Eine Neue Bestimming Der Molekuldimensionen." Ann. Phys. 1911, 34, 591. https://doi.org/10.1002/andp.19113390313.

(40) Taylor, G. I. The Viscosity of a Fluid Containing Small Drops of Another Fluid. Proc. R. Soc. A Math. Phys. Eng. Sci. 1932, 138, 41-48. https://doi.org/10.1098/rspa.1932.0169.

(41) Taylor, G. I. The Formation of Emulsions in Definable Fields of Flow. Proc. R. Soc. A Math. Phys. Eng. Sci. 1934, 146, 501-523. https://doi.org/10.1098/rspa.1934.0169.

(42) Oldroyd, J. G. The Elastic and Viscous Properties of Emulsions and Suspensions. Proc. R. Soc. London. Ser. A. Math. Phys. Sci. 1953, 218 (1132), 122-132. https://doi.org/10.1098/rspa.1953.0092.

(43) Oldroyd, J. G. The Effect of Interfacial Stabilizing Films on the Elastic and Viscous Properties of Emulsions. Proc. R. Soc. London. Ser. A. Math. Phys. Sci. 1955, 232 (1191), 567-577. https://doi.org/10.1098/rspa.1955.0240.

(44) de Bruijn, R. A. Deformation and Breakup of Drops in Simple Shear Flows, Eindhoven University of Technology, 1989. https://doi.org/10.6100/IR318702.

(45) Sondergaard, K.; Lyngaee-Jorgensen, J. Coalescence in an Interface-Modified Polymer Blend as Studied by Light Scattering. Polymer (Guildf). 1996, 37 (3), 509-517. https://doi.org/10.1016/0032-3861(96)82923-5.

(46) Gauthier, F.; Goldsmith, H. L.; Mason, S. G. Particle Motions in Non-Newtonian Media. Rheol. Acta 1971, 10, 344-364. https://doi.org/10.1007/BF01993709.

(47) Wu, S. Formation of Dispersed Phase in Incompatible Polymer Blends: Interfacial and Rheological Effects. Polym. Eng. Sci. 1987, 27 (5), 335-343. https://doi.org/10.1002/pen.760270506.

(48) Tomotika, S. Breaking up of a Drop of Viscous Liquid Immersid in Another Viscous Fluid Which Is Extending at a Uniform Rate. Proc. R. Soc. 1936, 153, 302-318. https://doi.org/10.1098/rspa.1936.0003. 
Controlling processing, morphology and mechanical performance in blends of polylactide and

thermotropic polyesters.

(49) Grace, H. P. Dispersion Phenomena in High Viscosity Immiscible Fluid Systems and Application of Static Mixers As Dispersion Devices in Such Systems. Chem. Eng. Commun. 1982, 14, 225-277. https://doi.org/10.1080/00986448208911047.

(50) Delaby, I.; Ernst, B.; Germain, Y.; Muller, R. Droplet Deformation in Polymer Blends during Uniaxial Elongational Flow: Influence of Viscosity Ratio for Large Capillary Numbers. J. Rheol. (N. Y. N. Y). 1994, 38 (6), 1705-1720. https://doi.org/10.1122/1.550568.

(51) Delaby, I.; Ernst, B.; Froelich, D.; Muller, R. Droplet Deformation in Immiscible Polymer Blends during Transient Uniaxial Elongational Flow. Polym. Eng. Sci. 1996, 36 (12), 1627-1635. https://doi.org/10.1002/pen.10559.

(52) Huneault, M. A.; Shi, H.; Utracki, L. A. Development of Polymer Blend Morphology during Compounding in a Twin-Screw Extruder. Part IV: A New Computational Model with Coalescence. Polym. Eng. Sci. 1995, 35 (1), 115-127. https://doi.org/10.1002/pen.760350114.

(53) Elemans, P. H. M. Modelling of the Processing of Incompatible Polymer Blends, Eindhoven University of Technology, 1989. https://doi.org/10.6100/IR316189.

(54) Shi, H.; Utracki, L. A. Development of Polymer Blend Morphology During Compounding in a Twin-Screw Extruder. Part II : Theoretical Derivations. Polym. Eng. Sci. 1992, 32 (24), 1834-1845. https://doi.org/10.1002/pen.760322406.

(55) Utracki, L. A.; Shi, Z. H. Development of Polymer Blend Morphology during Compounding in a Twin-Screw Extruder. Part I: Droplet Dispersion and Coalescence. (A Review). Polym. Eng. Sci. 1992, 32 (24), 1824-1833. https://doi.org/10.1002/pen.760322405.

(56) Kamal, M. R.; Utracki, L. A.; Mirzadeh, A. Rheology of Polymer Alloys and Blends. In Polymer Blends Handbook, Volume 2; Utracki, L. A., Wilkie, C., Eds.; Spring Netherlands: Dordrecht, 2014; pp 726-853. https://doi.org/10.1007/978-94-007-6064-6.

(57) Bordereau, V.; Shi, Z. H.; Utracki, L. A.; Sammut, P.; Carrega, M. Development of Polymer Blend Morphology During Compounding in a Twin-Screw Extruder. Part III: Experimental Procedure and Preliminary Results. Polym. Eng. Sci. 1992, 32 (24), 18461856. https://doi.org/10.1002/pen.760322407.

(58) Wilsens, C. H. R. M.; Verhoeven, J. M. G. A.; Noordover, B. A. J.; Hansen, M. R.; Auhl, D.; Rastogi, S. Thermotropic Polyesters from 2,5-Furandicarboxylic Acid and Vanillic Acid: Synthesis, Thermal Properties, Melt Behavior, and Mechanical Performance. Macromolecules 2014, 47 (10), 3306-3316. https:/ / doi.org/10.1021/ma500433e.

(59) Anastasiadis, S. H.; Gancarz, I.; Koberstein, J. T. Interfacial Tension of Immiscible Polymer Blends: Temperature and Molecular Weight Dependence. Macromolecules 1988, 21 (10), 2980-2987. https://doi.org/10.1021/ma00188a015.

(60) Elemans, P. H. M.; Janssen, J. M. H.; Meijer, H. E. H. The Measurement of Interfacial Tension in Polymer/Polymer Systems: The Breaking Thread Method. J. Rheol. (N. Y. N. Y). 2002, 34 (8), 1311-1325. https://doi.org/10.1122/1.550087.

(61) Wu, S. Calculation of Interfacial Tension in Polymer Systems. J. Polym. Sci. Part C Polym. Symp. 1971, 34 (1), 19-30. https://doi.org/doi:10.1002/polc.5070340105.

(62) de Kort, G. W.; Leoné, N.; Stellamanns, E.; Auhl, D.; Wilsens, C. H. R. M.; Rastogi, S. Effect of Shear Rate on the Orientation and Relaxation of a Vanillic Acid Based Liquid Crystalline Polymer. Polymers (Basel). 2018, $10 \quad$ (9), 935. https://doi.org/10.3390/polym10090935.

(63) Langelaan, H. C. The Relaxation of Shear and Normal Stresses of Nematic Liquid Crystalline Polymers in Squeezing and Shear Flows. J. Rheol. (N. Y. N. Y). 1996, 40 (1), 107. https://doi.org/10.1122/1.550733. 
(64) Qian, C.; Baird, D. G. The Transient Shear Rheology of a Thermotropic Liquid Crystalline Polymer in the Super-Cooled State. Polym. (United Kingdom) 2016, 102, 63-72. https://doi.org/10.1016/j.polymer.2016.08.056.

(65) Onogi, S.; Asasda, T. Rheology and Rheo-Optics of Polymer Liquid Crystals. In Rheology; Astarita, G., Marrucci, G., Nicolais, L., Eds.; Springer: Boston, 1980; pp 127-147. https://doi.org/10.1007/978-1-4684-3740-9_9.

(66) Lovell, C. S.; Montes de Oca, H.; Farrar, D.; Ries, M. E.; Ward, I. M. Analysis and Modeling of the Mechanical Properties of Novel Thermotropic Polymer Biomaterials. $\begin{array}{lllll}\text { Polymer } & \text { (Guildf). } & \text { 2010, } & 51 & \text { (9), }\end{array}$ https://doi.org/10.1016/j.polymer.2010.02.047. 


\subsection{Supporting Information}

\section{Parameters for the critical capillary number ( $\left.\kappa_{\text {critical }}\right)$.}

The critical capillary number marks the conditions where a droplet in a flow field becomes instable and can break up. This value depends on the type of flow field. The parameters used to calculate $\varkappa_{\text {critical }}$ were taken from literature and are listed below.

Table S3.1 Constants for calculation of the critical capillary number for shear-and extensional flow fields.

\begin{tabular}{|c|c|c|c|c|c|}
\hline Flow-field & $\mathrm{c}_{1}$ & $\mathrm{c}_{2}$ & $\mathrm{c}_{3}$ & $\mathrm{c}_{4}$ & $\mathrm{c}_{5}$ \\
\hline Shear & -0.506 & -0.0994 & 0.124 & -0.115 & -0.611 \\
\hline Extension & -0.6485 & -0.02442 & 0.02221 & -0.00056 & -0.00645 \\
\hline \multicolumn{7}{c}{$\log \left(\kappa_{\text {critical }}\right)=c_{1}+c_{2} \cdot \log (\lambda)+c_{3} \cdot \log (\lambda)^{2}+\frac{c_{4}}{\log (\lambda)+c_{5}}$}
\end{tabular}

\section{Time-temperature superposition of PLLA.}

The relaxation time of the PLLA melt at the processing temperatures was determined using time-temperature superposition. The obtained master curve and shift factors are reported.

a

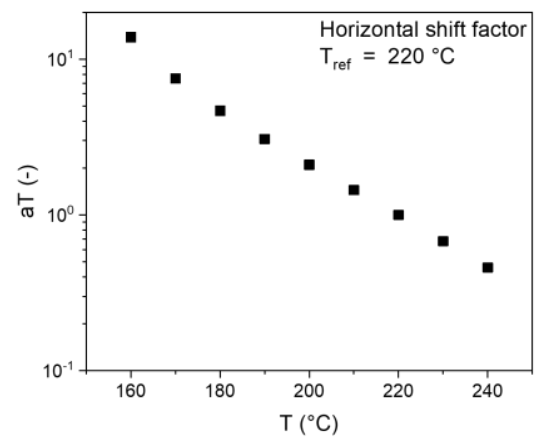

b

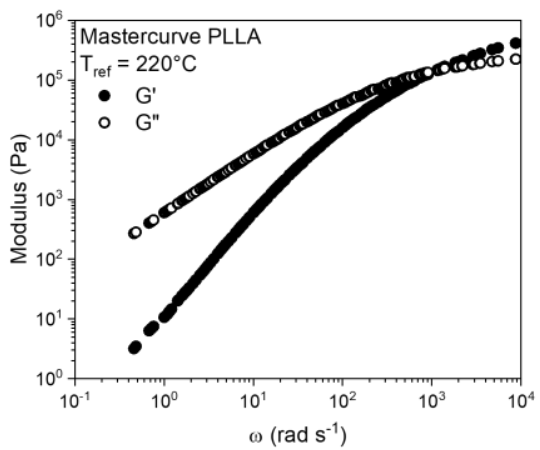

Figure S3.1 (a) Horizontal shift factors and (b) master curve obtained from time-temperature superposition of the PLLA (Corbion L130) used in this study at a reference temperature of $220^{\circ} \mathrm{C}$.

\section{Analyses of POM images of the blends in the barrel.}

In the injection molding process, the molten blend is extruded into a heated barrel prior to injection into the mold. The residence time in the barrel is typically $10 \mathrm{~s}$ to 1 min. During this stage of the process, the melt was not subjected to stresses, allowing stretched particles to relax. This process was mimicked by ex-situ heating the extrudate to the processing temperature. The resultant morphology was analyzed via POM, as the particles are easily distinguished upon dissolution of the PLLA matrix in a mixture of 
dichloromethane and acetone (ratio 3:1 by volume). The obtained images were processed and analyzed via ImageJ 1.50i. Several parameters describing the morphology are listed in Table S3.2, both the original POM images and the processed images are shown in Figures S3.2.the obtained particles size distributions (by number and by cumulative volume) are supplied in Figure S3.3.

Table S3.2 Morphological parameters of the blends during the barrel phase.

\begin{tabular}{|c|c|c|c|c|c|c|c|}
\hline & $\begin{array}{c}\mathrm{d}_{\mathrm{th}} \\
(\mu \mathrm{m})\end{array}$ & $\begin{array}{c}\mathrm{d}_{\min } \\
(\mu \mathrm{m})\end{array}$ & $\begin{array}{c}\mathrm{d}_{\max } \\
(\mu \mathrm{m})\end{array}$ & $\begin{array}{c}\mathrm{d}_{\text {avg }} \\
(\mu \mathrm{m})\end{array}$ & $\mathrm{V}_{\text {avg }}\left(\mu \mathrm{m}^{3}\right)$ & $\mathrm{c}_{\text {avg }}(-)$ & $\begin{array}{c}\mathrm{N}(- \\
)\end{array}$ \\
\hline $\begin{array}{c}10 \mathrm{wt}^{\%} \% \mathrm{LCP}- \\
\mathrm{A}\end{array}$ & 0.19 & 0.7 & 10.4 & $\begin{array}{c}2.9 \pm \\
2.0\end{array}$ & $\begin{array}{c}36.2 \pm \\
75.0\end{array}$ & $\begin{array}{c}0.75 \pm \\
0.14\end{array}$ & 150 \\
\hline $\begin{array}{c}30 \mathrm{wt} \% \text { LCP- } \\
\mathrm{A}\end{array}$ & 0.19 & 0.6 & 28.7 & $\begin{array}{c}3.0 \pm \\
3.4\end{array}$ & $\begin{array}{c}152 \pm \\
1025\end{array}$ & $\begin{array}{c}0.71 \pm \\
0.16\end{array}$ & 193 \\
\hline $\begin{array}{c}10 \mathrm{wt} \% \text { LCP- } \\
\mathrm{B}\end{array}$ & 0.17 & 0.3 & 3.5 & $\begin{array}{c}1.5 \pm \\
0.7\end{array}$ & $2.8 \pm 3.7$ & $\begin{array}{c}0.77 \pm \\
0.16\end{array}$ & 223 \\
\hline $\begin{array}{c}30 \mathrm{wt}^{\%} \% \mathrm{LCP}- \\
\mathrm{B}\end{array}$ & 0.17 & 0.6 & 9.0 & $\begin{array}{c}2.2 \pm \\
1.3\end{array}$ & $\begin{array}{c}12.8 \pm \\
30.6\end{array}$ & $\begin{array}{c}0.77 \pm \\
0.14\end{array}$ & 484 \\
\hline
\end{tabular}

$d_{t b}$ : theoretical minimum droplet diameter based on $x=\varkappa_{\text {critical. }}$. The measured viscosities and assumptions for the interfacial tension and shear rate during extrusion $\left(\nu_{12}=10 \mathrm{mN} \mathrm{m}^{-1}\right.$ and $\left.\gamma=100 \mathrm{~s}^{-1}\right)$ were used in the calculation.

$d_{\text {min: }}$ minimal droplet diameter (based on area).

$d_{\text {max }}$ : maximum droplet diameter (based on area).

$d_{\text {avg: }}$ average droplet diameter (based on area).

$V_{\text {avg: }}$ average droplet volume (based on area).

$c_{\text {avg: }}$ average circularity of droplets, where 1.0 is indicative of a perfect circle and values approaching 0.0 represent elongated polygons. circularity $=4 \pi$ (area/perimeter ${ }^{2}$ )

$N$ : number of droplets observed in POM image

Analysis of POM images was carried out via ImageJ $1.50 i$ (bttp:/ / imagej. nih.gov/ ij)

The images were converted to grayscale and subsequently a threshold was applied. The particles were analyzed via the 'Analyze Particles' function available in the software.
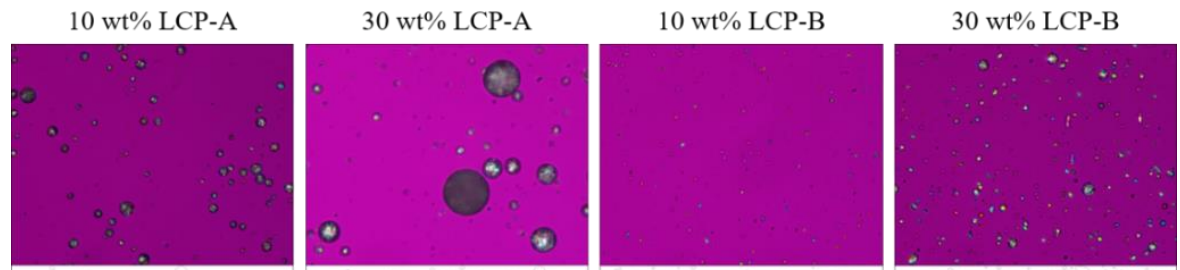

Figure S3.2 POM image (top) and processed image (bottom) of the different blends. 
Controlling processing, morphology and mechanical performance in blends of polylactide and thermotropic polyesters.

$\mathbf{a}$

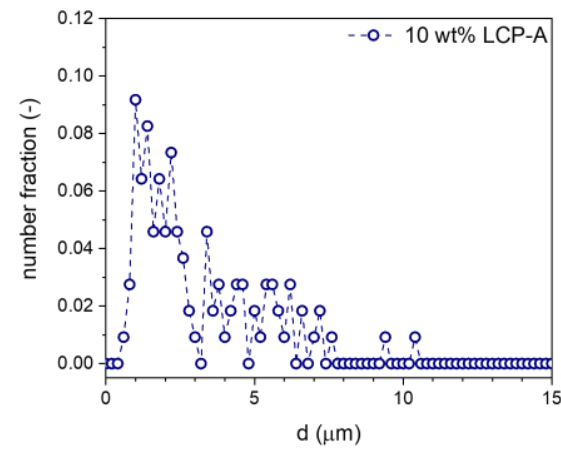

c

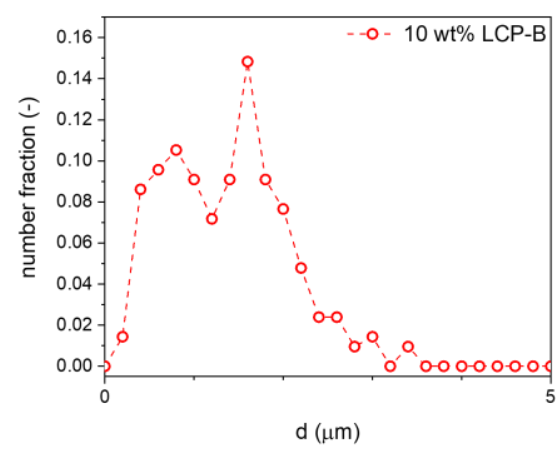

e

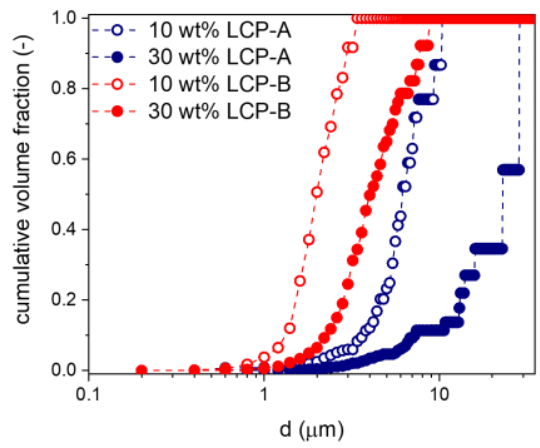

b

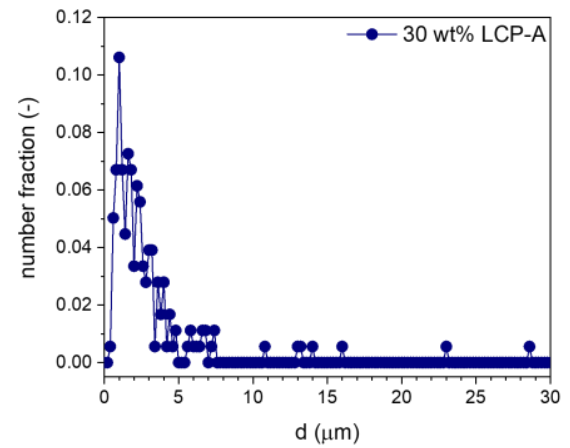

d

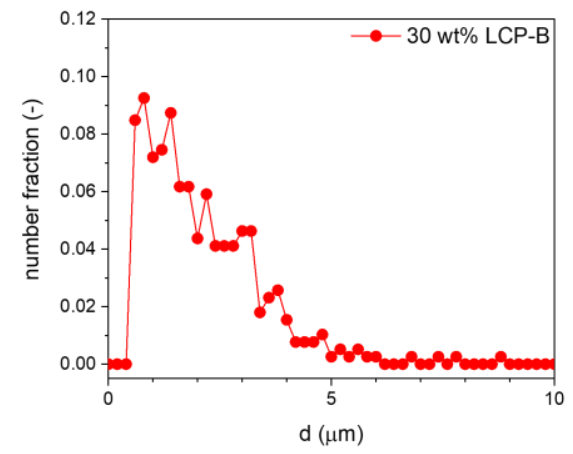

Figure S3.3 Particle size distributions by number of blends containting: (a)10 wt\% LCP-A, (b) $30 w t \%$ $L C P-A$, (c) $10 w t \%$ LCP-B, and (d) $30 w t \%$ LCP-B. (e) Cumulative particle volume distributions of all the blends. 


\section{Microstructure of injection molded samples containing $10 \mathrm{wt} \% \mathrm{LCP}$.}

Analogous to the injection molded samples containing $30 \mathrm{wt} \% \mathrm{LCP}$, the microstructure of the samples containing $10 \mathrm{wt} \%$ LCP were evaluated via SEM and WAXD (Figure S3.4)
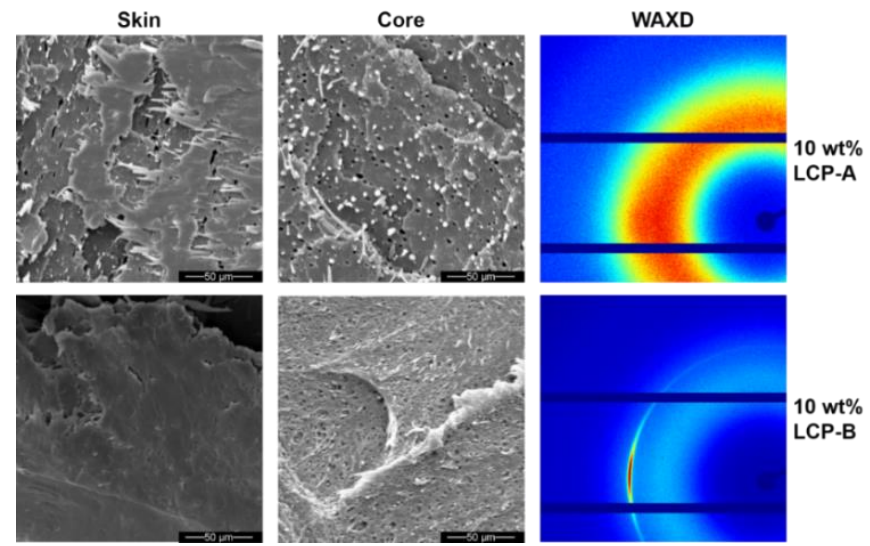

Figure S3.4 Microstructure of injection molded samples: SEM-images of sample skin (left, SEM-images of sample core (middle), and diffraction patterns (right).

\section{Comparison melt drawn and injection molded samples.}

We stated that an extensional flow field is the most effective route to morphology consisting of LCP fibrils with a high degree of interchain orientation. This is illustrated by the diffraction patterns shown in Figure S3.5. The LCP signal in the melt drawn tapes, in comparison to the injection molded samples, is more narrow and more intense with respect to the amorphous halo of the PLLA, indicating a higher degree of interchain orientation. Additionally, the diffraction patterns of the $30 \mathrm{wt} \% \mathrm{LCP}$ B/PLLA blend show a signal corresponding to isotropic LCP crystallites for the injection molded sample, whereas this signal is absent in the melt drawn tape.
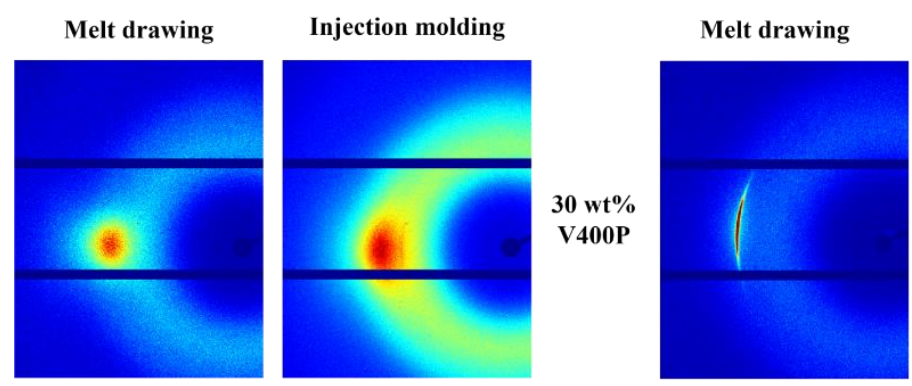

Injection molding

Figure S3.5 Diffraction patterns comparing the microstructure of melt drawn and injection molded LCP/PLLA composites containing $30 w t \%$ LCP. 


\title{
Chapter 4 - Thermoplastic PLA-LCP composites: a route towards sustainable, reprocessable, and recyclable reinforced materials.
}

\begin{abstract}
Reprocessing of reinforced composites is generally accompanied by loss of value and performance, as normally the reinforcing phase is damaged, or the matrix is lost in the process. In the search for more sustainable recyclable composite materials, we identify blends based on poly(L-lactide) (PLA) and thermotropic liquid crystalline polymers (LCP) as highly promising reinforced thermoplastic composites that can be recycled several times without any loss in the mechanical properties. For example, irrespective of the thermal history of the blend, injection molded bars of PLA containing $30 \mathrm{wt} \%$ LCP exhibit a tensile modulus of $6.4 \mathrm{GPa}$ and tensile strength around $110 \mathrm{MPa}$, as long as the PLA matrix has a molecular weight of $170 \mathrm{~kg} \mathrm{~mol}^{-1}$ or higher. However, after several mechanical reprocessing cycles, with the gradual decrease in molecular weight of the PLA matrix, deterioration of the mechanical performance is observed. The origin of this behavior is found in the increasing LCP to PLA viscosity ratio: At a viscosity ratio below unity, the LCP droplets are effectively deformed into the desired fibrillar morphology during injection molding. However, deformation of LCP droplets becomes increasingly challenging when the viscosity exceeds unity (i.e. when the PLA matrix viscosity decreases during consecutive reprocessing), eventually resulting in a nodular morphology, a poor molecular orientation of the LCP phase, and deterioration of the mechanical performance. This molecular weight dependency effectively places a limit on the maximum number of mechanical reprocessing cycles before chemical upgrading of the PLA phase is required. Therefore, a feasible route to maintain or enhance the mechanical properties of the blend, independent of the number of reprocessing cycles is proposed.
\end{abstract}

Published as:

de Kort, G. W.; Bouvrie, L.; Rastogi, S.; Wilsens, C. H. R. M. Thermoplastic PLA-LCP Composites: A Route towards Sustainable, Reprocessable, and Recyclable Reinforced Materials. Sustain. Chem. Eng. 2020, $8,624-631$. 


\subsection{Introduction}

Plastics are an omnipresent class of materials in our current society, useful in numerous applications, though their end-of-life options require improvement: It is unacceptable for non-biodegradable plastic waste to accumulate in our environment, neither is it desirable for plastic to accumulate in landfill. Instead, mechanical recycling, chemical recycling or energy recovery of plastics are more preferable and sustainable solutions ${ }^{1-5}$; The most preferred route is material specific and dependent on the degree of contamination ${ }^{6-8}$ and degradation ${ }^{9-11}$. Nevertheless, it is desirable that the chosen solution provides a closed-loop, thereby minimizing both the lost value and generated waste. Generally, routes towards circularity approach the issue from a chemical point of view ${ }^{12}$. However, as we will demonstrate in this work, there is also significant untapped potential in mechanical recycling of materials, given that all relevant physical parameters are well understood. For example, as we will demonstrate in this work, mechanical recycling of thermoplastic composites without loss of mechanical performance can be achieved by proper identification of relevant parameters (i.e. viscosity ratio) and boundary conditions (i.e. molecular weight limitations).

Compared to the recycling of thermoplastic polymers, recycling of reinforced composites poses an additional challenge: the composite properties are strongly dependent on the reinforcing phase. During mechanical recycling, the reinforcing phase, often brittle glass- or carbon fibers, is damaged and broken up, causing a decrease in mechanical performance. ${ }^{13-15}$ Solvolysis processes have been developed that can reclaim the reinforcing fibers; however, these involve large quantities of solvent and lead to loss of the matrix phase. ${ }^{16,17}$ In contrast, since the mechanical performance of the composites is determined by the morphology formed during melt processing, thermoplastic composites are promising alternatives to fiber-reinforced composites, providing mechanical reinforcement while allowing for mechanical recycling at their end-of-life. Therefore, in this work we focus on the mechanical reprocessing of thermoplastic reinforced composites consisting of poly(L-lactide) (PLA) as matrix and an aromatic thermotropic main-chain liquid crystalline polymer (LCP) as reinforcing filler.

Thermotropic LCP's are a suitable reinforcing phase for such composites as they are known to orient on a molecular level during flow, effectively enhancing their mechanical performance. ${ }^{18,19}$ This class of materials was intensively studied during the later decades of the last century, and has, due to its unique properties, received renewed interest due to its potential in 3D-printing ${ }^{20}$, as strong bio-resorbable materials ${ }^{21}$, and in sustainable composites $^{22}$. When dispersed in a thermoplastic matrix and upon the application of 
flow, LCPs can form elongated fibrils, effectively generating reinforced composites..$^{23,24}$ The dispersion and morphology of LCP particles and chain orientation are key parameters for the performance of LCP-reinforced composites. Therefore, the thermaland flow-behavior of both phases and the processing conditions should be taken into account as these parameters determine the morphology of the dispersed LCP phase ${ }^{25}$.

The high processing temperature of most commercial LCP's (generally over $300{ }^{\circ} \mathrm{C}$ ) can be a limiting factor, as most thermoplastic matrices have limited stability in this temperature range. For example, PLA, as a polyester, is susceptible to degradation during processing, being sensitive to both hydrolysis and thermal degradation at elevated temperatures, resulting in a decrease in molecular weight. ${ }^{26,27}$ Therefore, a commercially available, amorphous LCP is used, which can be processed at temperatures as low as $220^{\circ} \mathrm{C}$.

\subsection{Methods and Materials}

\section{Materials}

The PLA grades used in this study were purchased from Total Corbion (Purapol LX175 $\left(M_{\mathrm{w}}=245 \mathrm{~kg} \mathrm{~mol}^{-1}, \mathrm{D}\right.$-content $\left.=4 \%\right)$, Luminy L130 $\left(M_{\mathrm{w}}=170 \mathrm{~kg} \mathrm{~mol}^{-1}, \mathrm{D}\right.$ content $<1 \%$ ), and Purapol L105 $\left(M_{\mathrm{w}}=153 \mathrm{~kg} \mathrm{~mol}^{-1}\right.$, D-content $\left.<1 \%\right)$, abbreviated from here onwards as PLA-245, PLA-170, and PLA-153 respectively. Additionally, the Synterra PLLA 1010 grade produced by Synbra $\left(M_{\mathrm{w}}=122 \mathrm{~kg} \mathrm{~mol}^{-1}\right.$, D-content $\left.<1 \%\right)$ is used and abbreviated as PLA-122. The thermotropic polyester used in this study was purchased from Celanese (Vectra LCP ${ }^{\circledR}$ V400P, recommended processing temperature $220-240{ }^{\circ} \mathrm{C}$ ), and is abbreviated from here onwards to LCP. Vectra ${ }^{\circledR}$ LCP V400P grade is a registered trademark of Celanese Corporation, Dallas, TX. The material composition, as well as the results associated with this material are the property of Celanese Corporation.

\section{Preparation and Processing of PLA-LCP Blends}

The as obtained pellets (abbreviated as sample "V" for later analysis) were dried overnight at $60{ }^{\circ} \mathrm{C}$ in vacuo prior to use. Mixtures of the respective PLA and LCP pellets were prepared in the following compositions: $0 \mathrm{wt} \%$ LCP (pure PLA), $10 \mathrm{wt} \% \mathrm{LCP}$, and $30 \mathrm{wt} \%$ LCP. The granulate mixtures were processed in the molten state using a Collin ZK 25T Teach Line twin-screw extruder at a temperature of $220{ }^{\circ} \mathrm{C}$ and a screw speed of $100 \mathrm{rpm}$, and subsequently chopped into pellets. These one-time extruded pallets are abbreviated as sample "E" for later analysis.

The produced blends were molded into dogbones $(2 \mathrm{~mm} \times 4 \mathrm{~mm} \times 80 \mathrm{~mm}$, with a gauge length of $30 \mathrm{~mm}$ ) via injection molding. A BOY XS twin-screw injection molder 
was used. The melt- and mold temperatures were set to $220^{\circ} \mathrm{C}$ and $45^{\circ} \mathrm{C}$, respectively, with an injection pressure of $100 \mathrm{bar}$. After analysis, the dogbones were dried in vacuo $\left(60{ }^{\circ} \mathrm{C}\right.$, overnight $)$ and ground prior to reprocessing. The injection molded samples are abbreviated based on the number of reprocessing cycles; For example, the material that was injection molded for the first time is named " $\mathrm{R}_{0}$ ", whereas the dogbones that were recycled 7 times are named " $\mathrm{R} 7$ ". The $30 \mathrm{wt} \%$ LCP composites based on PLA-245, PLA-170, PLA-153, PLA-122, and blends of PLA-245 and PLA-170 (in ratios of 100:0, 75:25, 50:50, 25:75, and 0:100) were produced via the same procedure. The PLA-LCP composite based on PLA-245 with extended extrusion time (PLA-245-E15) was extruded for 15 minutes in a DSM Xplore twin-screw micro-extruder $\left(220^{\circ} \mathrm{C}, 100 \mathrm{rpm}\right)$ with a recycle prior to injection molding.

\section{Material Characterization.}

The viscoelastic behavior of the pure materials was determined in a MCR 702 TwinDrive rheometer (Anton Paar) with a parallel plate geometry (diameter of $12 \mathrm{~mm}$, gap of $0.7 \mathrm{~mm}$ ). Samples were loaded at a temperature of $190{ }^{\circ} \mathrm{C}$, and frequency sweeps were carried out with a strain of $1 \%$.

The mechanical properties of the composites were assessed via tensile testing, performed on a Zwick Z100. Samples were subjected to a constant deformation rate of $5 \mathrm{~mm} \mathrm{~min}^{-1}$, at room temperature.

The molecular weight distributions of the PLA were obtained via Gel Permeation Chromatography (GPC). A Shimadzu LC-2030 system was used with chloroform as solvent, the obtained values are relative to a poly(styrene) standard. Prior to injection, all samples were passed through a PTFE membrane filter with a pore size of $0.2 \mu \mathrm{m}$. The PLAs molecular weight distribution could be determined in the case of PLA-LCP composites, as the LCP was found to be insoluble in chloroform and was eliminated from the sample upon filtration.

The microstructure of the composites was assessed via a combination of Scanning Electron Microscopy (SEM) and Polarized Optical Microscopy (POM). A Philips X30 microscope was used for the SEM-imaging of the fracture surfaces, at an acceleration voltage of $15 \mathrm{kV}$ and magnifications of 150x and 1000x. The fracture surfaces were created by breaking samples cooled in liquid nitrogen, which were subsequently mounted and plasma-coated with a thin layer of gold. An Olympus BX53 Microscope (20x magnification) equipped with an Olympus DP26 camera was used for optical microscopy. To display the LCP particle morphology, the PLA-phase was dissolved in chloroform, a non-solvent for the LCP. 


\subsection{Results and Discussion}

The performance of an LCP reinforced thermoplastic composite is mainly determined by the morphology; a fibrillar reinforcing phase with a high aspect ratio is desired 1) to achieve a high molecular orientation required for high tensile modulus and strength of the dispersed LCP phase, and 2) to effectively transfer stresses from matrix to filler and prevent preliminary failure..$^{23,28-31}$ In this study, we use commercially available polymers, being thermotropic polyester Vectra LCP V400P (LCP) and several polylactides. As explained in the experimental section the $M_{\mathrm{w}}$ (weight average molecular weight) of the PLA grades are $245 \mathrm{~kg}$ mole $^{-1}$ (PLA-245), $170 \mathrm{~kg} \mathrm{~mole}^{-1}$ (PLA-170), $153 \mathrm{~kg} \mathrm{~mole}^{-1}$ (PLA153), and $122 \mathrm{~kg} \mathrm{~mole}^{-1}$ (PLA-122). The PLA and LCP are processed together via extrusion followed by injection molding.

During the extrusion step, the LCP is dispersed in the PLA matrix through droplet breakup facilitated by the complex combination of shear and extensional flow in the extruder 32,33 : Deformation of droplets occurs as the capillary number ( $x$, Eq.1), the ratio of the hydrodynamic- and interfacial forces, exceeds unity. In the case the capillary number is larger then a critical value ( $x_{\text {critical }}$, Figure 4.1$)$, which depends on the flow type and viscosity ratio ( $\lambda$, Eq. 2 ), the droplet stretches until $x$ equals the critical value and breaks up into smaller droplets as a result of the decreasing diameter. Given a sufficient amount of extrusion time, an equilibrium particle distribution is obtained as the LCP particle size will decrease until particle breakup is balanced by coalescence.

After extrusion, the blend is injection molded where it is subjected to high shear, and cooling rates. During this process, the capillary number and the viscosity ratio increase (as illustrated in Figure 4.1a) due to the imposed cooling and the increased in deformation rate. Given that LCP droplets can only stretch into fibrils when $x>>x_{\text {critical }}$, the resultant morphology and aspect ratio depend on the original viscosity ratio (and consequently on the $M_{\mathrm{w}}$ of the PLA matrix). Stable stretching of droplets is achieved at $\lambda \leq 1$ (marked in solid green in Figure 4.1a), whereas at higher $\lambda$ droplet deformation is increasingly hindered. In the high $\lambda$ regime, cooling and flow do initially facilitate droplet deformation to a smaller extent (marked in striped green in Figure 4.1a), but as the viscosity ratio increases further the droplets can no longer be stretched (marked in red in Figure 4.1a). In the latter case the LCP droplets, having a $x$ smaller then $x_{\text {critical }}$, are rotated by shear flow, instead of stretched. To be more precise, the timescale required for the extensional component to stretch a droplet exceeds the timescale for rotation: the moment a droplet deforms slightly it rotates to minimize the stress it experiences and subsequently relaxes back into a sphere. This does not allow the formation of fibrils, and this is the inherent meaning of the asymptote at $\lambda=3.8$ in 
Figure 4.1a. The increasing viscosity ratio upon cooling enhances this effect further although coalescence can still occur. More in depth information on the topics of blend morphology and droplet deformation is provided in Chapter 3.

$$
\begin{aligned}
& \kappa=\frac{\eta_{\text {matrix }} \cdot \dot{\gamma} \cdot d}{v_{12}} \\
& \lambda=\frac{\eta_{L C P}}{\eta_{\text {matrix }}}
\end{aligned}
$$

a

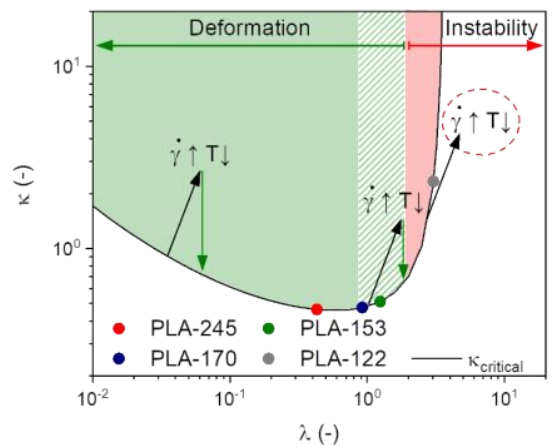

c
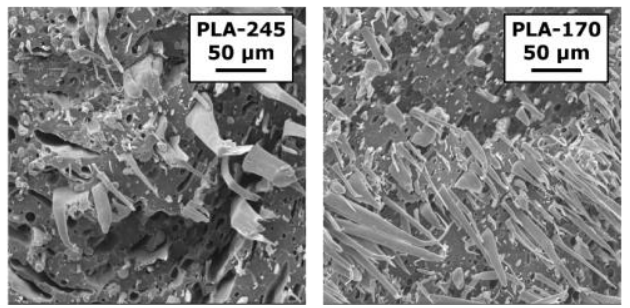

b
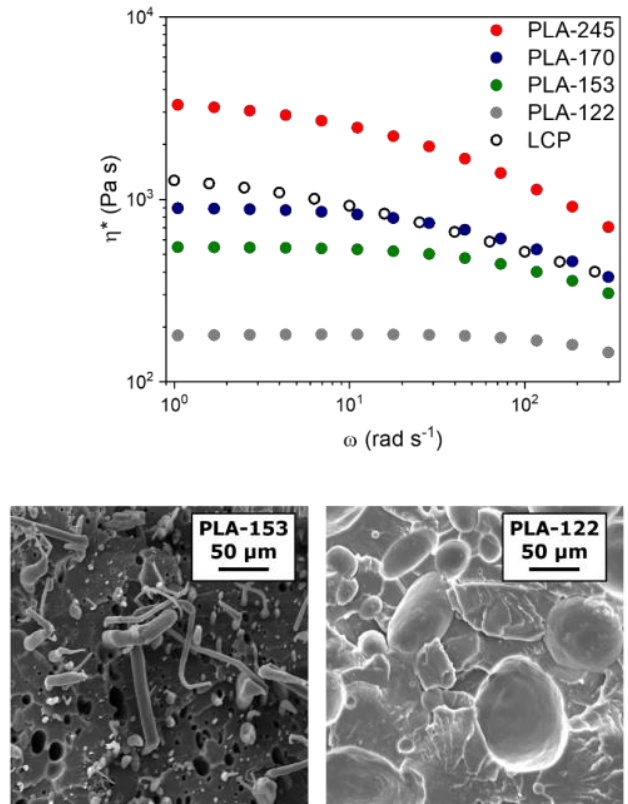

Figure 4.1 (a) Capillary number as a function of viscosity ratio for a shear flow field. The general effect of injection molding on the capillary number and viscosity ratio is illustrated by the arrows and different regimes with respect to the deformability of the LCP droplets are marked. Note that the points marking the observed viscosity ratios for the different $P L A$ 's shift from the region where LCP drops are easily deformed into the instable region as the $M_{w}$ of the PLA decreases. (b) Viscosities of blend components measured via rheometry at $190{ }^{\circ} \mathrm{C}$. (c) Observed morphology of composites containing $30 \mathrm{wt} \%$ LCP with different matrix $M_{w}$.

Figure 4.1b displays the complex viscosity as a function of the angular frequency for the various PLA grades and the LCP. From this data, the viscosity ratio $\lambda$ can be determined at any given angular frequency, and the corresponding values (at $\omega=100$ $\operatorname{rad~s}{ }^{-1}$, assuming the Cox-Merz relation holds) are highlighted in Figure 4.1a, left. Conform theory, one can expect that the use of a decreasing molecular weight of PLA makes it more difficult to stretch LCP particles during injection molding, in particular 
for the PLA-122 sample. As mentioned earlier, under these conditions, a flow field as experienced in injection molding, having a considerable shear component, will simply result in the rotation and coalescence of droplets instead of deformation into fibrils ${ }^{25}$. Indeed, this is confirmed by analysing the fracture morphology of the injection molded blends containing LCP and the different PLA grades in SEM analysis (Figure 4.1c). For the blends containing PLA-245, PLA-170, and PLA-153, a predominantly fibrillar LCP morphology is observed. As expected from predictions, the LCP phase in the blend containing PLA-122 only exhibits spherical or poorly oriented LCP particles. The effect of these changes on the orientation of the LCP is shown in the Supporting Information (Figure S4.1). The implications of these variations in morphology on the mechanical properties are addressed in a later section.

a

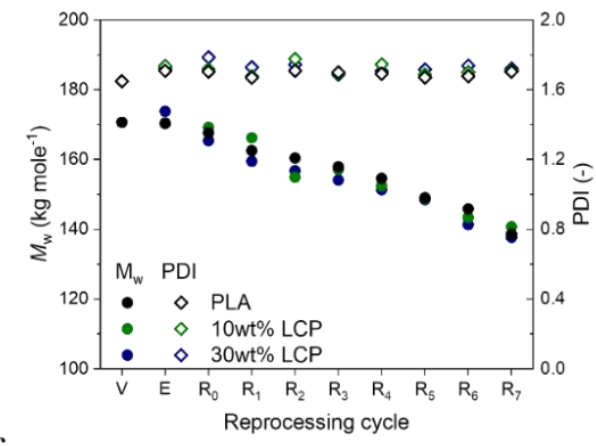

b

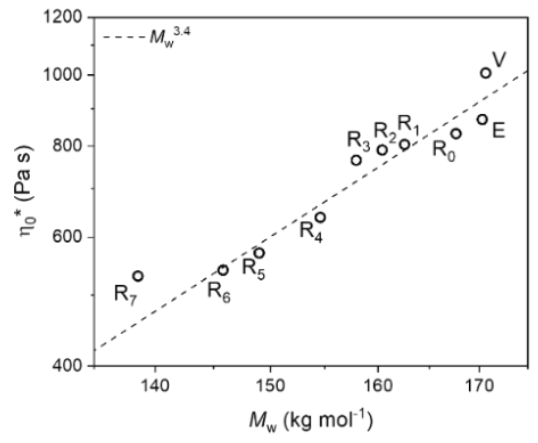

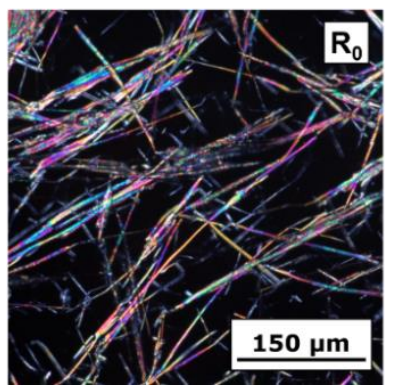
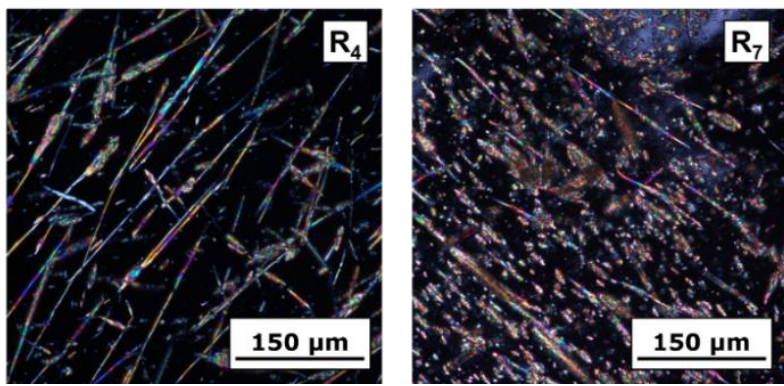

Figure 4.2 (a) Weight average molecular weight and polydispersity of PLA for reprocessed PLA and reprocessed composites. (b) Zero-shear viscosity as function of molecular weight for reprocessed PLA-170. Frequency sweep data is available in Figure S4.2 of the Supporting Information. (c) Changes in LCP particle morphology over the course of reprocessing, POM images taken under crossed-polarizers, PLA was selectively dissolved in chloroform.

It is well-known that PLA is susceptible to degradation processes in the melt ${ }^{34,35}$, which can affect the material properties after consecutive reprocessing or recycling steps. In fact, the decrease in molecular weight is accompanied by a gradual drop in melt 
viscosity, mechanical properties, and an acceleration in crystallization kinetics over the course of several reprocessing cycles ${ }^{26,27}$. As explained in the previous section, PLA degradation and the concomitant decrease in viscosity results in an increase in $\lambda$ of the PLA-LCP blends and is thereby expected to influence both composite morphology and the mechanical performance. To investigate the effect of reprocessing on the molecular weight of the PLA matrix, a series of samples have been prepared consisting of PLA170 , and either 0,10 , or $30 \mathrm{wt} \%$ LCP. These samples have been analysed in the virgin state, prior to processing (labeled V), after a one-time extrusion to create the blends (labeled $\mathrm{E}$ ), after first-time injection molding $\left(\mathrm{R}_{0}\right)$, and after reprocessing for up to 7 cycles $\left(\mathrm{R}_{1}\right.$ to $\left.\mathrm{R}_{7}\right)$. The molecular weight $\left(\mathrm{M}_{\mathrm{w}}\right)$, zero shear viscosity $\left(\eta_{0}{ }^{*}\right)$, and LCP particle morphology have been evaluated after each cycle. An overview is provided in Figure 4.2 .

Figure 4.2a shows the evolution of the molecular weight $\left(M_{\mathrm{w}}\right)$ and polydispersity index (PDI) of the PLA matrix, obtained from the pure PLA-170 and the PLA-LCP composites after each (re)processing cycle. As expected, we observe a gradual decrease of the molecular weight, though no change in the molecular weight distribution is observed upon consecutive reprocessing. Overall, the $M_{w}$ decreases by roughly $18 \%$ over the course of 7 cycles for all samples. Furthermore, it is clear that the presence of LCP as the dispersed phase does not influence the degradation process of the PLA phase during reprocessing. Corresponding to the decrease in molecular weight, the viscosity decreases with each processing or reprocessing step (Figure 4.2b). The zeroshear viscosity $\left(\eta_{0}^{*}\right.$, determined from parallel plate rheometry at $190{ }^{\circ} \mathrm{C}$, frequency sweep data is available in Figure S4.2 of the Supporting Information) is shown as a function of $M_{\mathrm{w}}$ (determined by GPC), and follows the expected scaling for entangled polymer melts $\left(\eta_{0}^{*} \sim M_{w}{ }^{3.4}\right)$. It is noteworthy that the molecular weight and viscosity of the PLA170 gradually decreases during consecutive reprocessing to a value somewhere between PLA-153 and PLA-122. As previously explained, this affects the obtained morphology: The gradual decrease in $M_{\mathrm{w}}$ of the PLA matrix upon reprocessing increases the viscosity ratio $(\lambda)$, which limits the deformation and molecular orientation (as shown in Figure S4.1) of the dispersed LCP particles during injection molding. The viscoelastic response of the LCP remained unaffected by reprocessing, as shown in Figure S4.3 of the Supporting Information. Figure 4.2c shows the morphology of PLA-LCP blends containing $10 \mathrm{wt} \%$ LCP after a different number of reprocessing cycles. The original morphology $\left(\mathrm{R}_{0}\right)$ is dominated by homogeneous fibrils with a high aspect ratio. After four reprocessing cycles $\left(\mathrm{R}_{4}\right)$, the fibrils appear less homogeneous and an additional population of droplets with a low aspect ratio has appeared. Finally, after seven cycles $\left(\mathrm{R}_{7}\right)$ the morphology is dominated by slightly deformed droplets, while poorly oriented 
and inhomogeneous fibrils form a secondary group of particles. The LCP particle morphologies observed in polarized optical microscopy are in line with the aforementioned theory regarding droplet behavior, as they confirm that the decrease in $M_{\mathrm{w}}$ of the PLA phase results in a gradual increase in $\lambda$ and thereby alters the deformability of the LCP particles during injection molding.

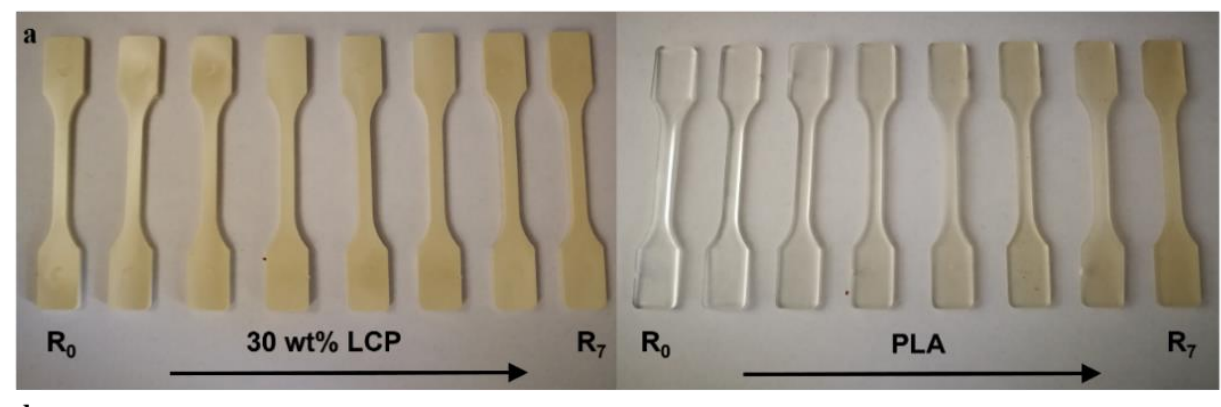

b

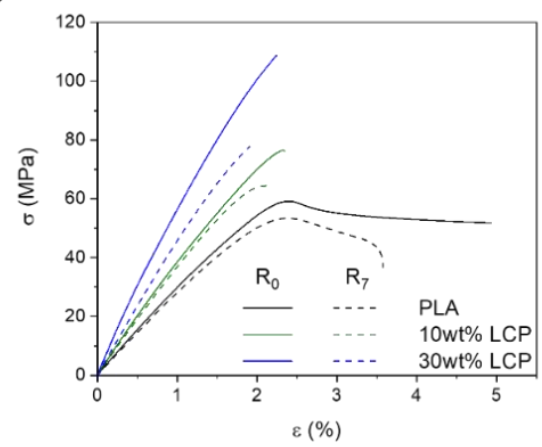

c

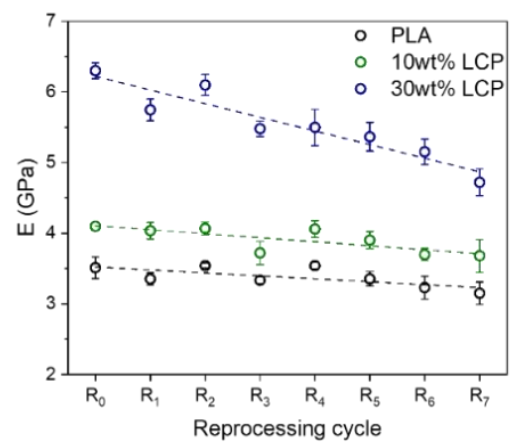

Figure 4.3 (a) Visual appearance of tensile bars after a specified number of reprocessing cycles. (b) Characteristic stress-strain curves for the fresh materials and the materials after 7 reprocessing cycles. (c) Young's Modulus of the reprocessed samples.

An interesting difference between the reprocessed PLA and the reprocessed PLALCP composites arises from their respective visual appearances (Figure 4.3a). The amorphous PLA is initially transparent, but gradually becomes hazy due to the increasing presence of crystallites and contaminants. The PLA-LCP composites, on the other hand, are opaque with a beige color and do not visually change upon reprocessing. The dispersed LCP fibrils, with particle sizes larger than the wavelengths of visible light, scatter light and thus dominate the sample appearance. This feature can be considered as beneficial from an aesthetic perspective, at least, compared to the reprocessed PLA samples.

The mechanical performance is an important characteristic of reinforced composites and therefore Figure $4.3 \mathrm{~b}$ displays the tensile curves of $\mathrm{R}_{0}$ and $\mathrm{R}_{7}$ samples with varying 
LCP content. In general, we observe an effective reinforcement of the PLA by the LCP phase, as the modulus and stress at break readily increase with LCP content. However, as the PLA decreases in molecular weight and the LCP morphology changes, a clear decrease in mechanical performance is observed. This is clearly exemplified by the decrease of the Young's modulus (E) of both the PLA and the PLA-LCP blends over the course of seven reprocessing cycles (Figure 4.3c). The modulus of the pure PLA decreases gradually upon repeated reprocessing, though the overall change is limited $(<10 \%)$. In contrast, the Young's modulus of the $30 \mathrm{wt} \%$ LCP composites decreases with $25 \%$ after 7 reprocessing cycles. A similar trend was observed with respect to the maximum stress, as shown in Figure S4.4 of the Supporting Information.

$\mathbf{a}$

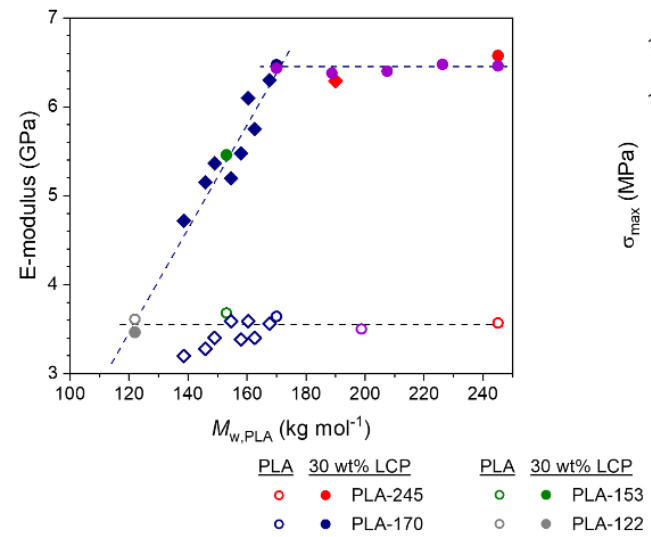

b

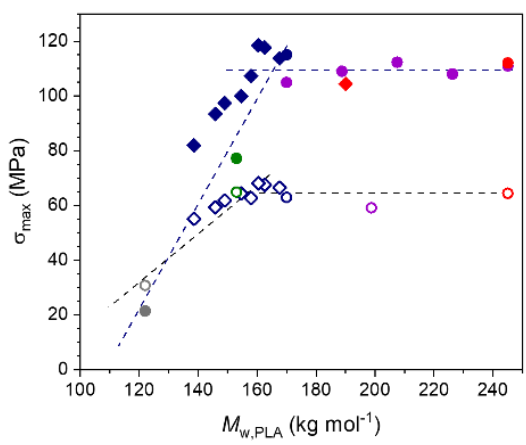

$$
\begin{aligned}
\frac{\text { PLA }}{\circ} & \frac{30 \text { wt } \% \text { LCP }}{\bullet} \\
\diamond & \text { Blend } \\
\diamond & \text { Reprocessed } \\
& \text { PLA-245-E15 }
\end{aligned}
$$

Figure 4.4 (a) Young's modulus of PLA and $30 w t \%$ LCP composites as function of matrix Mw. (b) Maximum stress of PLA and $30 w t \%$ LCP composites as function of matrix Mw. The yield stress was used as the maximum stress for the pure PLA samples; the stress at break was used as the maximum stress for the 30 $w t \%$ PLA composites.

Figure 4.4a shows an overview of the Young's moduli of the injection molded dogbones of PLA and PLA-LCP blends (30 wt \%) as a function of the molecular weight $\left(M_{\mathrm{w}}\right)$ of PLA matrix. In this plot, data from composites based on 1) different virgin matrices (PLA-245 to PLA-122), 2) reprocessed PLA-170 ( $\mathrm{R}_{0}$ to $\left.\mathrm{R}_{7}\right)$, 3) blends of PLA245 and PLA-170 in different ratios, and 4) PLA-245 that was extruded for 15 minutes to mimic several reprocessing cycles (PLA-245-E15), are combined. In the case of the pure PLA samples, the modulus remains largely unaffected by $M_{\mathrm{w}}$, indicating that the decrease in mechanical properties upon reprocessing of pure PLA is likely correlated to the accumulation of impurities, or degradation of additives. The PLA-LCP blends show an interesting trend: the Young's modulus of the blends remain constant for samples a PLA matrix with a $M_{\mathrm{w}}$ of $170 \mathrm{~kg} \mathrm{~mol}^{-1}$ and higher, whereas samples with a $M_{\mathrm{w}}$ below 
$170 \mathrm{~kg} \mathrm{~mol}^{-1}$ exhibit a decreasing modulus. Whether the composites were reprocessed or not, the relation between the modulus and the $M_{\mathrm{w}}$ of the matrix remains the same. The maximum tensile stress (Figure 4.4b), shows the same trend with a constant value when the PLA matrix has a $M_{\mathrm{w}}$ of $170 \mathrm{~kg} \mathrm{~mol}^{-1}$ and higher, while decreasing when the PLA molecular weight drops below this value. This confirms that the mechanical performance of PLA-LCP composites, irrespective of the thermal history, depends mainly on the molecular weight of the PLA phase for the given processing conditions.

These two regimes, in combination with the fact that the mechanical performance of the reprocessed composites matched with the other samples, have strong implications for the recycling of PLA-LCP composites, and potentially thermoplastic composites in general. Our results show that PLA-LCP composites can be reprocessed numerous times without deterioration of the mechanical properties, given that the molecular weight of the PLA remains sufficiently high to allow deformation of the LCP droplets during injection molding. This reprocessability is a unique and highly desirable property in order to achieve more sustainable reinforced composites. Given that rheological behavior of the currently used LCP grade, Vectra LCP V400P, changes little under the evaluated processing conditions even after prolonged exposure to a temperature of $220^{\circ} \mathrm{C}$, it appears that the degradation of the PLA sample is the limiting factor in the reprocessing process described above. In other words, the use of high molecular weight PLA is desired as starting material. Note that, choice of the PLA and LCP grade (with their respective molecular weights and viscosities) used to form the composite, determine the extent to which the molecular weight of the PLA is allowed to decrease until recycling without deterioration of the mechanical performance is no longer possible. Therefore, the $\lambda$ of the initially produced blend is preferably around 0.1 .

Overall, this bottom-up understanding for the development of reinforced thermoplastic composites that are reprocessed without loss of performance has large implication on their recyclability. However, as the molecular weight of the PLA matrix decreases with each cycle (due to both reprocessing and exposure during use), at some point the morphology of the composite, and correspondingly the performance, will deteriorate. This means that this process does not yet allow for closed-loop recycling, as the composite, or rather the PLA matrix eventually has to be discarded or upgraded. There are, however, well-known methods available to increase the molecular weight of PLA, which can provide the requirements to achieve the desired closed-loop recycling process, as is illustrated in Figure 4.5. One approach is Solid State Post Condensation (SSPC), a technique widely used in polycondensates to increase the molecular weight of polymer after the initial polymerization, and it has been utilized in numerous studies to 
obtain PLA with a molecular weight well above that used in this study. ${ }^{38}$ Another option is depolymerization of PLA into lactide as a product ${ }^{39}$. In turn, the reclaimed lactide can be used as feed to conduct a ring-opening polymerization (ROP), once again yielding high molecular weight PLA ${ }^{40}$. When required, separation of the LCP particles from the PLA phase can readily be achieved by filtration after dissolution of the PLA in common laboratory solvents such as tetrahydrofuran or chloroform, which are non-solvents for the LCP phase. Additional resources required in these processes, such as energy and chemicals, increase the ecological footprint of the overall process. The production of lactide via chemical depolymerization of PLA requires less energy compared to production of lactide via the fermentation of glucose ${ }^{39}$. The increased environmental impact due to the need to maintain a minimum PLA molecular weight diminishes quickly, however, considering that the PLA produced in these processes is of a high enough molecular weight and the composites performance can be maintained over multiple thermomechanical recycling steps.

Lastly, it is noteworthy to highlight that the deformation, breakup and coalescence of droplets in a matrix, are mainly governed by physical parameters and are not specific to the chemical composition of the blend. ${ }^{32}$ The findings and insight reported in this study are not necessarily limited to the combination of PLA and LCP, as the theoretical framework holds for any thermoplastic blend. A similar window, where the performance can be maintained upon thermomechanical recycling, is likely to be available in other non-miscible thermoplastic composites, as long as the thermal and physical behavior of both phases matches.

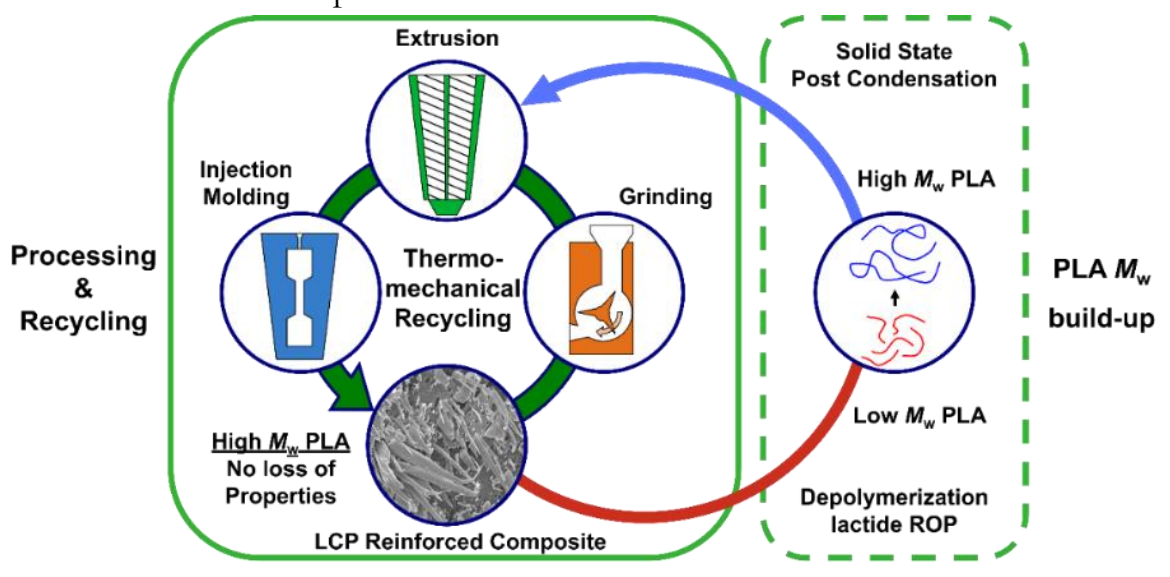

Figure 4.5 Overview of strategies for reprocessing (left) and enhancing the molecular weight of PLA (right). Connecting these strategies provides a route towards closed-loop recycling of LCP reinforced composites. Extension of the work conducted on thermomechanical recycling with either SSPC or full chemical recycling of the PLA phase allows an indefinite number of use cycles without loss of mechanical properties. 


\subsection{Conclusions}

We have demonstrated the reprocessability and excellent mechanical performance of thermoplastic composites containing PLA as a matrix and a thermotropic LCP as a reinforcing phase. The mechanical performance of the composites appears strongly dependent on the viscosity ratio between the two components as this governs the resultant LCP morphology after injection molding. However, due to thermal degradation from processing, the molecular weight of the PLA matrix is found to decrease gradually with each reprocessing cycle, resulting into a successive decrease in the LCP particle orientation and retrospectively decreasing the mechanical performance of the composite. The molecular weight of the PLA matrix is identified as the dominant parameter in this process, as data from composites with different PLA grades and varying molecular weights matches excellently with recycled composites.

Two distinct regimes, dependent on the LCP/PLA viscosity ratio are identified: In the first regime $(\lambda<1)$, an increase in matrix viscosity results in a slightly coarser morphology, however, the overall mechanical performance is not effected as the LCP is still present in the form of long, thin fibrils, and the degree of LCP orientation remains high. In the second regime $(\lambda>1)$, a decrease in matrix viscosity results in less favorable LCP morphology as the matrix is less effective in deforming the molten LCP. At high viscosity ratio $(\lambda \sim 3.8)$, causes in a complete lack of droplet deformation by a shear flow field: the LCP is present in the form of large nodules. In the second regime, the mechanical performance decreases at lower matrix molecular weight.

In the regime where the viscosity ratio of the blend is low $(\lambda<1)$, the mechanical performance of melt-processable LCP/PLA composites does not decrease upon recycling. This regime can be obtained readily from the thermal- and flow behavior of the LCP- and matrix phase. As basis for this regime stems from the flow behavior and morphology development, it is a generic feature of blends and not limited to LCP/PLA systems. These findings might prove useful in the search for recyclable and sustainable composite materials. 


\subsection{References}

(1) Al-Salem, S. M.; Lettieri, P.; Baeyens, J. Recycling and Recovery Routes of Plastic Solid Waste (PSW): A Review. Waste Manag. 2009, 29 (10), 2625-2643. https://doi.org/10.1016/j.wasman.2009.06.004.

(2) Rahimi, A. R.; Garciá, J. M. Chemical Recycling of Waste Plastics for New Materials Production. Nat. Rev. Chem. 2017, 1, 1-11. https://doi.org/10.1038/s41570-017-0046.

(3) Perugini, F.; Mastellone, M. L.; Arena, U. A Life Cycle Assessment of Mechanical and Feedstock Recycling Options for Management of Plastic Packaging Wastes. Environ. Prog. 2005, 24 (2), 137-154. https://doi.org/10.1002/ep.10078.

(4) Asmatulu, E.; Twomey, J.; Overcash, M. Recycling of Fiber-Reinforced Composites and Direct Structural Composite Recycling Concept. J. Compos. Mater. 2014, 48 (5), 593-608. https://doi.org/10.1177/0021998313476325.

(5) Shuaib, N. A.; Mativenga, P. T. Energy Demand in Mechanical Recycling of Glass Fibre Reinforced Thermoset Plastic Composites. J. Clean. Prod. 2016, 120, 198-206. https://doi.org/10.1016/j.jclepro.2016.01.070.

(6) Luijsterburg, B.; Goossens, H. Assessment of Plastic Packaging Waste: Material Origin, Methods, Properties. Resour. Conserv. Recycl. 2014, 85, 88-97. https://doi.org/10.1016/j.resconrec.2013.10.010.

(7) Jansen, M.; Thoden van Velzen, U.; Pretz, T. Handbook for Sorting of Plastic Packaging W aste Concentrates S; 2015.

(8) Luijsterburg, B. J.; Jobse, P. S.; Spoelstra, A. B.; Goossens, J. G. P. Solid-State Drawing of Post-Consumer Isotactic Poly(Propylene): Effect of Melt Filtration and Carbon Black on Structural and Mechanical Properties. Waste Manag. 2016, 54, 53-61. https://doi.org/10.1016/j.wasman.2016.04.029.

(9) Teteris, G. Degradation of Polyolefines during Various Recovery Processes. Macromol. Symp. 1999, 144, 471-479. https://doi.org/10.1002/masy.19991440143.

(10) Jin, H.; Gonzalez-Gutierrez, J.; Oblak, P.; Zupančič, B.; Emri, I. The Effect of Extensive Mechanical Recycling on the Properties of Low Density Polyethylene. Polym. Degrad. $\begin{array}{llll}\text { Stab. 2012, } & 97 & \text { (11), } & \end{array}$ https://doi.org/10.1016/j.polymdegradstab.2012.07.039.

(11) Choudhury, A.; Mukherjee, M.; Adhikari, B. Thermal Stability and Degradation of the Post-Use Reclaim Milk Pouches during Multiple Extrusion Cycles. Thermochim. Acta 2005, 430 (1-2), 87-94. https:/ / doi.org/10.1016/j.tca.2004.12.025.

(12) Christensen, P. R.; Scheuermann, A. M.; Loeffler, K. E.; Helms, B. A. Closed-Loop Recycling of Plastics Enabled by Dynamic Covalent Diketoenamine Bonds. Nat. Chem. 2019, 11 (5), 442-448. https://doi.org/10.1038/s41557-019-0249-2.

(13) Eriksson, P. A.; Albertsson, A. C.; Boydell, P.; Prautzsch, G.; Månson, J. A. E. Prediction of Mechanical Properties of Recycled Fiberglass Reinforced Polyamide 66. Polym. Compos. 1996, 17 (6), 830-839. https://doi.org/10.1002/pc.10676.

(14) Kuram, E.; Tasci, E.; Altan, A. I.; Medar, M. M.; Yilmaz, F.; Ozcelik, B. Investigating the Effects of Recycling Number and Injection Parameters on the Mechanical Properties of Glass-Fibre Reinforced Nylon 6 Using Taguchi Method. Mater. Des. 2013, 49, 139-150. https://doi.org/10.1016/j.matdes.2013.02.027.

(15) Davies, P.; Le Duigou, A.; Pillin, I.; Baley, C.; Bourmaud, A. Effect of Recycling on Mechanical Behaviour of Biocompostable Flax/Poly(l-Lactide) Composites. Compos. Part A Appl. Sci. Manuf. 2008, 39 (9), 1471-1478. https://doi.org/10.1016/j.compositesa.2008.05.008. 
Thermoplastic PLA-LCP composites: a route towards sustainable, reprocessable, and recyclable reinforced materials.

(16) Morin, C.; Loppinet-Serani, A.; Cansell, F.; Aymonier, C. Near- and Supercritical Solvolysis of Carbon Fibre Reinforced Polymers (CFRPs) for Recycling Carbon Fibers as a Valuable Resource: State of the Art. J. Supercrit. Fluids 2012, 66, 232-240. https://doi.org/10.1016/j.supflu.2012.02.001.

(17) Goto, M.; Sasaki, M.; Tokuno, S.; Shibata, K.; Iwaya, T. Recycling of Fiber Reinforced Plastics Using Depolymerization by Solvothermal Reaction with Catalyst. J. Mater. Sci. 2007, 43 (7), 2452-2456. https://doi.org/10.1007/s10853-007-2017-8.

(18) Chung, T.-S. The Recent Developments of Thermotropic Liquid Crystalline Polymers. Polym. Eng. Sci. 1986, 26 (13), 901-919. https://doi.org/10.1002/pen.760261302.

(19) Acierno, D.; Mantia, F. P. La; Polizzotti, G.; Ciferri, A.; Valenti, B. Ultrahigh Modulus Liquid Crystalline Polyesters. Macromolecules 1982, 15 (6), 1455-1460. https://doi.org/10.1021/ma00234a001.

(20) Gantenbein, S.; Masania, K.; Woigk, W.; Sesseg, J. P. W.; Tervoort, T. A.; Studart, A. R. Three-Dimensional Printing of Hierarchical Liquid-Crystal-Polymer Structures. Nature 2018, 561 (7722), 226-230. https://doi.org/10.1038/s41586-018-0474-7.

(21) Montes de Oca, H.; Wilson, J. E.; Penrose, A.; Langton, D. M.; Dagger, A. C.; Anderson, M.; Farrar, D. F.; Lovell, C. S.; Ries, M. E.; Ward, I. M.; et al. Liquid-Crystalline Aromatic-Aliphatic Copolyester Bioresorbable Polymers. Biomaterials 2010, 31 (30), 7599-7605. https://doi.org/10.1016/j.biomaterials.2010.07.006.

(22) de Kort, G. W.; Rastogi, S.; Wilsens, C. H. R. M. Controlling Processing, Morphology, and Mechanical Performance in Blends of Polylactide and Thermotropic Polyesters. Macromolecules 2019, 52, 6005-6017. https://doi.org/10.1021/acs.macromol.9b01083.

(23) Kiss, G. In Situ Composites: Blends of Isotropic Polymers and Thermotropic Liquid Crystalline Polymers. Polym. Eng. Sci. 1987, 27 (6), 410-423. https://doi.org/10.1002/pen.760270606.

(24) DeMeuse, M. T.; Kiss, G. Liquid Crystal Polymers (LCPs) as a Reinforcement in High Temperature Polymer Blends; Woodhead Publishing Limited, 2014. https://doi.org/http://dx.doi.org/10.1533/9780857099013.165.

(25) Utracki, L. A.; Shi, Z. H. Development of Polymer Blend Morphology during Compounding in a Twin-Screw Extruder. Part I: Droplet Dispersion and Coalescence. (A Review). Polym. Eng. Sci. 1992, 32 (24), 1824-1833. https://doi.org/10.1002/pen.760322405.

(26) Pillin, I.; Montrelay, N.; Bourmaud, A.; Grohens, Y. Effect of Thermo-Mechanical Cycles on the Physico-Chemical Properties of Poly(Lactic Acid). Polym. Degrad. Stab. 2008, 93 (2), 321-328. https:// doi.org/10.1016/j.polymdegradstab.2007.12.005.

(27) Badia, J. D.; Strömberg, E.; Karlsson, S.; Ribes-Greus, A. Material Valorisation of Amorphous Polylactide. Influence of Thermo-Mechanical Degradation on the Morphology, Segmental Dynamics, Thermal and Mechanical Performance. Polym. $\begin{array}{lllll}\text { Degrad. Stab. } & \text { 2012, } & 97 & \text { (4), }\end{array}$ https://doi.org/10.1016/j.polymdegradstab.2011.12.019.

(28) Bassett, B. R.; Yee, A. F. A Method of Forming Composite Structures Using in Situ Formed Liquid Crystal Polymer Fibers in a Thermoplastic Matrix. Polym. Compos. 1990, 11 (1), 10-18. https://doi.org/10.1002/pc.750110103.

(29) Blizard, K. G.; Baird, D. G. The Morphology and Rheology of Polymer Blends Containing a Liquid Crystalline Copolyester. Polym. Eng. Sci. 1987, 27 (9), 653-662. https://doi.org/10.1002/pen.760270909.

(30) Blizard, K. G.; Federici, C.; Federico, O.; Chapoy, L. L.; Caduti, V. The Morphology of Extruded Blends Containing a Thermotropic Liquid Crystalline Polymer. Polym. Eng. Sci. 1990, 30 (22), 1442-1453. https://doi.org/10.1002/pen.760302205. 
(31) Silverstein, M. S.; Hiltner, A.; Baer, E. Hierarchical Structure in LCP/PET Blends. J. Appl. Polym. Sci. 1991, 43 (1), 157-173. https://doi.org/10.1002/app.1991.070430113.

(32) Kamal, M. R.; Utracki, L. A.; Mirzadeh, A. Rheology of Polymer Alloys and Blends. In Polymer Blends Handbook, Volume 2; Utracki, L. A., Wilkie, C., Eds.; Spring Netherlands: Dordrecht, 2014; pp 726-853. https://doi.org/10.1007/978-94-007-6064-6.

(33) Heino, M. T.; Hietaoja, P. T.; Vainio, T. P.; Seppälä, J. V. Effect of Viscosity Ratio and Processing Conditions on the Morphology of Blends of Liquid Crystalline Polymer and Polypropylene. J. Appl. Polym. Sci. 1994, 51 (2), 259-270. https://doi.org/10.1002/app.1994.070510208.

(34) Auras, R. Poly(Lactic Acid) Synthesis, Structures, Properties, Processing, and Application; Auras, R., Lim, L., Selke, S., Tsuji, H., Eds.; John Wiley \& Sons, Inc.: Hoboken, 2010. https://doi.org/10.1017/CBO9781107415324.004.

(35) Dorgan, J. R.; Janzen, J.; Clayton, M. P.; Hait, S. B.; Knauss, D. M. Melt Rheology of Variable L-Content Poly(Lactic Acid). J. Rheol. (N. Y. N. Y). 2005, 49 (3), 607. https://doi.org/10.1122/1.1896957.

(36) Thomason, J. L.; Vlug, M. A. Influence of Fibre Length and Concentration on the Properties of Glass Fibre-Reinforced Polypropylene: 1. Tensile and Flexural Modulus. Compos. Part A Appl. Sci. Manuf. 1996, 27 (6), 477-484. https://doi.org/10.1016/1359835X(95)00065-A.

(37) Thomason, J. L.; Vlug, M. A.; Schipper, G.; Krikor, H. G. L. T. Influence of Fibre Length and Concentration on the Properties of Glass Fibre-Reinforced Polypropylene: Part 3. Strength and Strain at Failure. Compos. Part A Appl. Sci. Manuf. 1996, 27 (11), 1075-1084. https://doi.org/10.1016/1359-835X(96)00066-8.

(38) Peng, B.; Hou, H.; Song, F.; Wu, L. Synthesis of High Molecular Weight Poly(1 -Lactic Acid) via Melt/Solid State Polycondensation. II. Effect of Precrystallization on Solid State Polycondensation. Ind. Eng. Chem. Res. 2012, 51 (14), 5190-5196. https://doi.org/10.1021/ie202192q.

(39) Piemonte, V.; Sabatini, S.; Gironi, F. Chemical Recycling of PLA: A Great Opportunity Towards the Sustainable Development? J. Polym. Environ. 2013, 21 (3), 640-647. https://doi.org/10.1007/s10924-013-0608-9.

(40) Nijenhuis, A. J.; Grijpma, D. W.; Pennings, A. J. Lewis Acid Catalyzed Polymerization of L-Lactide. Kinetics and Mechanism of the Bulk Polymerization. Macromolecules 1992, 25 (24), 6419-6424. https://doi.org/10.1021/ma00050a006. 


\subsection{Supporting Information}

\section{D-WAXD diffraction patterns and orientation parameter as function of matrix} Mw.
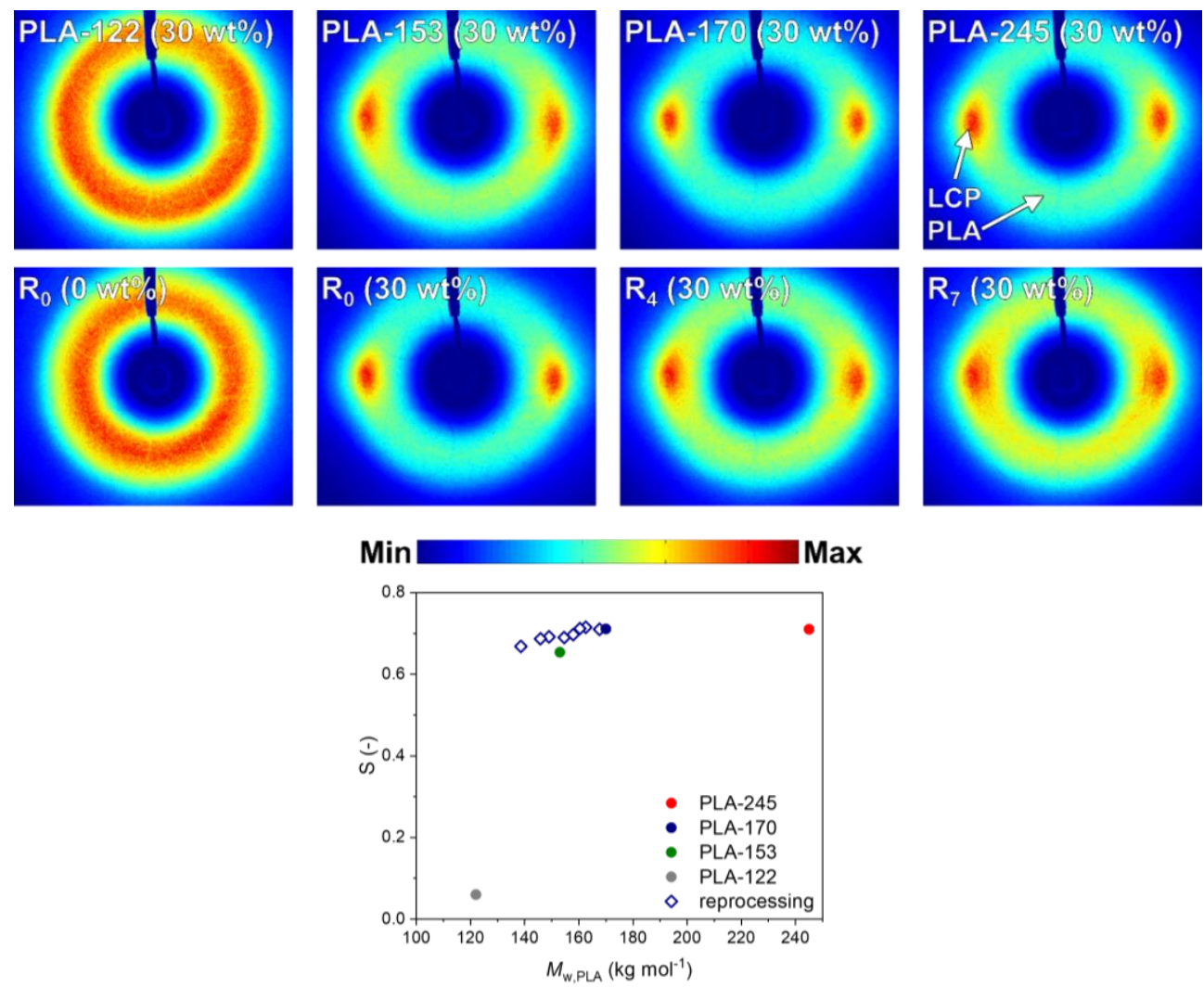

Figure S4.12D-WAXD diffraction patterns of several samples, flow direction is vertical in the image-plane (top). Orientation parameter $S$ as a function of matrix $M_{w}$, as calculated from the diffraction patterns. The orientation parameter was corresponds mainly to the degree of interchain orientation of the LCP, where $S=1$ signifies perfect alignment and $S=0$ signifies a completely isotropic state (bottom).

All diffraction patterns, with the exception of PLA-122 (30 wt $\%)$ and $\mathrm{R}_{0}(0 \mathrm{wt} \%)$, consist of two superimposed signals: an isotropic amorphous halo, (PLA phase), and two amorphous arcs, (oriented LCP phase, as marked in PLA-245 (30 wt \%)). The magnitude of the color difference between both signals is an indication for the level of LCP orientation: a larger difference in color between the two signals (PLA-245 (30 $\left.\mathrm{wt}^{\mathrm{O}} \%\right)$ ) indicates, whereas a smaller color difference signifies a slightly lower level of orientation (PLA-153 (30 wt \%)). The diffraction patterns of PLA-122 (30 wt $\%$ )and $\mathrm{R}_{0}$ $\left(0 \mathrm{wt}^{\%} \%\right)$ both show an isotropic amorphous halo. In however, in the case PLA-122 
$(30 \mathrm{wt} \%)$, the highest signal intensity is obtained at higher diffraction angles. This is due the overlap between the signals of both the isotropic components, and the LCP having a signal at a larger diffraction angle. The diffraction pattern of $\mathrm{R}_{7}(30 \mathrm{wt} \%)$ shows two additional faint arcs at a lower angle (towards the center of the image), which indicates the presence of a small amount of oriented PLA crystallites. This change crystallization behavior of the PLA can be attributed either to enhanced nucleation resulting from the accumulation of impurities, or to the presence of some fraction of non-linear chains, as indicated in Figures S4.2.

The bottom figure shows the orientation parameter $\mathrm{S}$, signifying the degree of interchain orientation in the LCP, as a function of the molecular weight of the PLA matrix. At high PLA $M_{\mathrm{w}}$ the LCP is oriented. A decrease in $M_{\mathrm{w}}$ initially does not result in a change in S. Lowering $M_{\mathrm{w}}$ further, below $170 \mathrm{~kg} \mathrm{~mol}^{-1}$, leads to a gradual decrease in orientation, and at some point even a completely isotropic state. This is in accordance with the morphologies of the composites obtained via SEM and POM, and corresponds with the observed mechanical performance. This confirms that the LCP is oriented when the dispersed LCP droplets are deformed and stretched during processing.

Wide-angle X-ray Diffraction (WAXD) measurements (wave length, $\lambda=0.104 \mathrm{~nm}$ ) were carried out at the European Synchrotron Radiation Facility (ESRF), the DUBBLE beamline (BM26B, Grenoble, France). The orientation parameter $(S),\left\langle\mathrm{P}_{2 \mathrm{n}}(\cos \varphi)\right\rangle_{\mathrm{d}}$, was calculated from the obtained diffraction patterns via a procedure by Mitchell and Windle, as described in Chapter 2.

\section{Complex viscosity of PLA-170 after each reprocessing cycle.}

Figure S4.2 shows that the molecular weight of the PLA gradually decreases over the course of multiple reprocessing cycles, as the zero-shear viscosity gradually decreases and the onset of shear-thinning gradually shifts to higher frequencies. Conform the GPC data, rheometry indicates no changes in the shape of the molecular weight distribution, with the exception of $\mathrm{R}_{7}$. After 7 reprocessing cycles, a small deviation from this behavior is observed: at the low frequency side of the curve, there is a slight upturn in the viscosity after it had seemingly reached its plateau value $\left(\eta_{0}^{*}\right)$. This is an indication of either the formation of a small amount of long chain branches, or the presence of network of a particulate nature due to contamination resulting from reprocessing. 


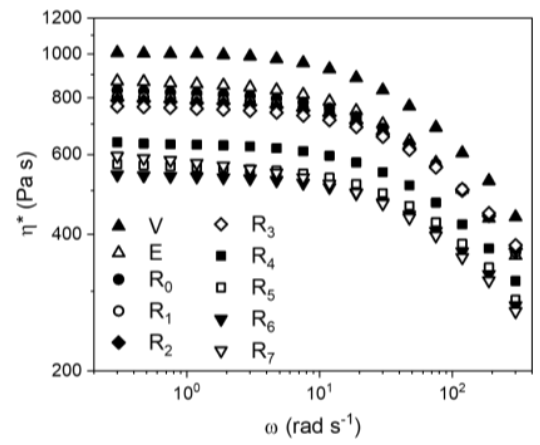

Figure S4.2 Complex viscosity as a function of oscillation frequency of PLA-170 (0 wt\% LCP) after each reprocessing cycle. $\mathrm{T}=190^{\circ} \mathrm{C}$.

\section{Stability of the LCP under the applied reprocessing conditions.}

a

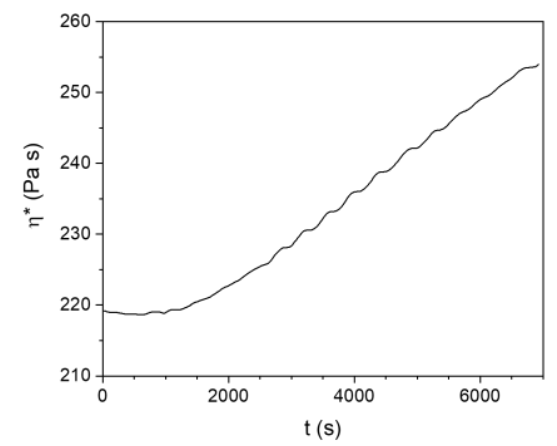

b

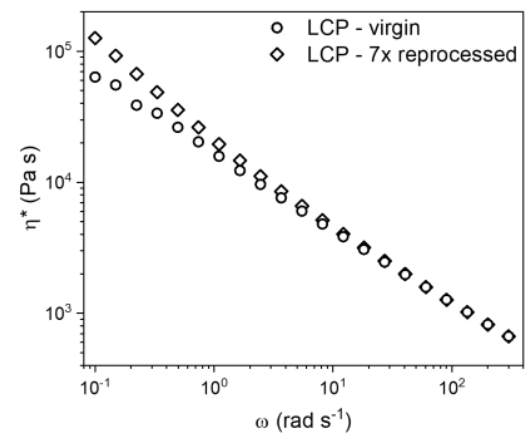

Figure S4.3 (a) Complex viscosity of the thermotropic LCP as a function of time. $T=220{ }^{\circ} \mathrm{C}, \omega=10 \mathrm{rad} \mathrm{s}^{-1}$. (b) The complex viscosity of virgin LCP and LCP recovered after 7 reprocessing cycles plotted versus the angular frequency. Measured at $200^{\circ} \mathrm{C}$.

The LCP was subjected elevated temperatures for a significant period in a plate-plate rheometer, in order to assess the likelihood of changes in the viscoelastic response over several reprocessing cycles (Figure S4.3a). Assuming a residence time of 180 seconds at the processing temperature per cycle, the cumulative residence time is 2000 to 2500 seconds, corresponding to a minor variation in the LCP viscosity. It should be noted that changes might be accelerated under actual processing conditions, but a large change in LCP viscosity over the course of several reprocessing cycles remains unlikely.

To ensure that the viscoelastic behavior or the LCP remains similar upon reprocessing of the composites, the LCP was isolated and characterized after the last reprocessing step $\left(\mathrm{R}_{7}\right)$. The reprocessed LCP was recovered via dissolution of the PLA matrix in chloroform, followed by filtration and drying. Even though the LCP has been 
reprocessed 7 times, the viscoelastic response (Figure S4.3b), especially in the high frequency regime, remained identical to that of the original material. Due to limitations of the applied filtration procedure, a small amount of PLA is still present in the reprocessed LCP. The observed differences in the viscoelastic behavior at low frequencies could be caused by the response of small, dispersed PLA droplets. Therefore, changes in the flow behavior of the LCP are expected to have no effect on the morphological development.

Note that there is a discrepancy between the viscoelastic response of the LCP reported here and elsewhere in this work. This is due to differences in the experimental set-up, as a different parallel plate set-up was used (plate diameter $=25 \mathrm{~mm}$, gap $=0.5$ $\mathrm{mm}$, strain $=0.5 \%$ ). The viscoelastic response of LCPs is complex and highly sensitive to such changes, as it depends on the polydomain texture of the material during the actual measurement.

\section{Development of the maximum tensile stress over the course of seven reprocessing cycles.}

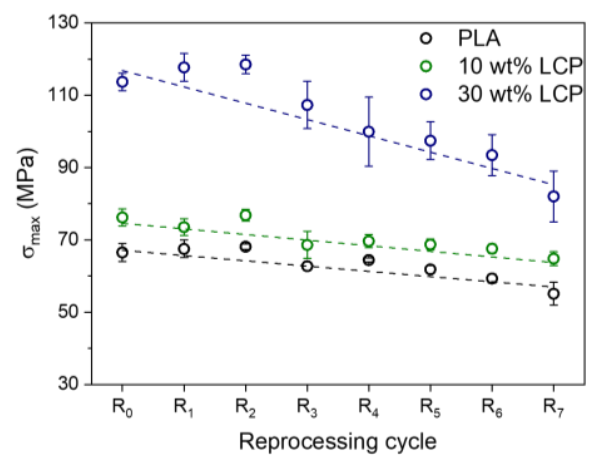

Figure S4.4 Development of the maximum tensile stress over the course of seven reprocessing cycles. The maximum stress corresponds to the stress at break in the case of PLA-LCP composites and to the yield stress in the case of pure PLA.

The LCP proved to be effective in reinforcing the PLA, as is confirmed by the increase in maximum stress in Figure S4.4. This effect was more significant at higher LCP-content, and persisted over the course of the seven reprocessing cycles. However, over the course of seven reprocessing cycles the maximum stress was found to drop. Decreases in stress of $28 \%, 15 \%$, and $18 \%$ were found for the materials containing 30 $\mathrm{wt}^{0} \%, 10 \mathrm{wt} \%$, and $0 \mathrm{wt} \%$ LCP, respectively. For most samples, a relatively large decrease was observed over the course of the seventh cycle, as the drop in stress over the first six cycles corresponds to $18 \%, 11 \%$, and $11 \%$, respectively. 
The yield stress is reported for the pure PLA, whereas the stress at break is reported for the PLA-LCP composites. This was done in order not to over-emphasize the reinforcing effect of the LCP. It was not possible to report the yield stress for all samples, as the PLA-LCP composites did not yield.

\section{Thermal behavior of LCP and PLA-170}

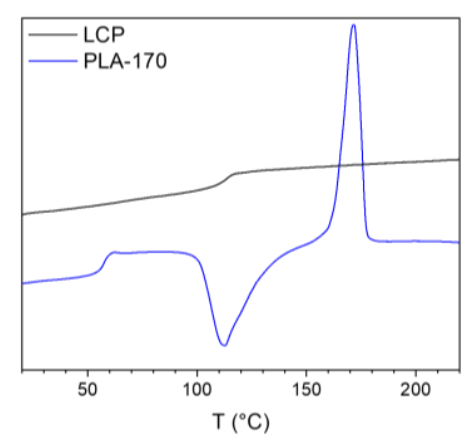

Figure S4.5 Differential scanning calorimetry thermograms of the second heating step of LCP and PLA-170, respectively. Measurements were carried out at a beating $10^{\circ} \mathrm{C} \mathrm{min}^{-1}$ (endo up).

The thermal behavior of LCP and PLA-170, measured via differential scanning calorimetry, are shown in Figure S4.5. LCP has a high $T_{\mathrm{g}}$ around $110^{\circ} \mathrm{C}$, and does not display a clear melting peak. This behavior is expected for a fully aromatic copolymer, which does not exhibit extensive formation of non-periodic layer crystallites. PLA-170 grade crystallizes slowly, as indicated by the presence of a large cold crystallization peak during heating. PLA-170 exhibits a $\mathrm{T}_{\mathrm{g}}$ around $60^{\circ} \mathrm{C}$ and a peak melting temperature of $175^{\circ} \mathrm{C}$. 
Chapter 4 


\title{
Chapter 5 - A reactive processing route to thermotropic polyesters with low processing temperature and enhanced relaxation time.
}

\begin{abstract}
The processing temperature of thermotropic LCPs can be reduced by increasing the flexibility of the backbone, simultaneously decreasing the relaxation time and resulting in a decrease in the mechanical properties in the processed polymer. Here we report on the development of thermotropic liquid crystalline polymers (LCPs) with low processing temperatures, increased relaxation time, and excellent mechanical performance via a versatile two-step synthesis. These LCPs can be processed at temperatures below $200{ }^{\circ} \mathrm{C}$ into injection molded parts which have a mechanical performance comparable to that of commercially available grades with Young's modulus and tensile strength over 12.5 GPa and $200 \mathrm{MPa}$, respectively. These LCPs are synthesized using carboxylic acid terminated thermotropic prepolymers that are produced via a standard acidolysis polycondensation at $220{ }^{\circ} \mathrm{C}$, a temperature that allows incorporation of monomers having lower thermal stability. Subsequently, the prepolymers are subjected to a chain-extension reaction with bis(2-oxazoline)s in a reactive extrusion step. The facile two-step method provides excellent control over the chemical composition, molecular weight, and chain architecture of the resulting polymer. As the molecular weight of the LCPs increases, they display interesting and uncharacteristic viscoelastic behavior. The viscoelastic response of the LCPs changes from that of an unentangled melt, typically observed in rigid LCPs, towards a physically constrained melt indicated by the appearance of a crossover point with an increase in molar mass. Such a response is normally not observed in commercial thermotropic LCPs even with an increase in molar mass. We attribute change in the viscoelastic response to the chain extension reaction. This fundamental change in the state of the nematic melt greatly increases the relaxation timescales of both the interchain orientation and the nematic texture, providing further evidence towards their interrelation. The change in the relaxation mechanism of the nematic phase with increasing molar mass is followed by time resolved X-ray diffraction and optical microscopy.
\end{abstract}

Under review as:

de Kort, G. W.; Saidi, S.; Hermida-merino, D.; Leoné, N.; Rastogi, S.; Wilsens, C. H. R. M. A., Reactive Processing Route to Thermotropic Polyesters with Low Processing Temperature and Enhanced Relaxation Time., Macromolecules, 2020. 


\subsection{Introduction}

Thermotropic main-chain polyesters are an interesting class of materials, characteristically forming a nematic phase at elevated temperatures due to the presence of rigid mesogenic monomers into the backbone of a polymer ${ }^{1,2}$. In a thermotropic melt, orientation on a molecular level can be achieved and maintained by the application of a flow field followed by cooling, resulting in materials with a high tensile modulus and tensile strength ${ }^{3}$. In addition, these liquid crystalline polymers (LCPs) typically exhibit exceptional thermal and chemical stability, combined with low thermal expansion coefficients. These characteristic properties have not only resulted into the development of several commercially available thermotropic polyesters, generally targeting high performance applications, but also in the development of liquid crystalline thermosets ${ }^{4-6}$, 3D-printing processes for $\mathrm{LCPs}^{7}$, bio-resorbable $\mathrm{LCPs}^{8}$, and reprocessable reinforced LCP based composites?

These new applications, in combination with a societal push towards the more sustainable use of plastic materials, require a more versatile approach for the development of thermotropic polyesters. Such a versatile approach would need to facilitate a feasible route to include new monomers, combined with an effective way to control the molecular weight and chain architecture of the LCP.

The current (commercial) generation of LCPs combine good temperature resistance and excellent mechanical properties, making them highly suitable for high performance applications. Generally, conventional thermotropic polyesters are prepared via an acidolysis polycondensation of acetylated aromatic monomers at high temperatures ( $>$ $\left.280{ }^{\circ} \mathrm{C}\right)^{10}$. However, these stringent synthesis conditions result in challenges incorporating novel, rigid (bio-based) monomers, suffering from limited thermal stability, into the LCPs backbone ${ }^{11}$. Besides the limitations posed by high processing temperatures, a more stringent problem of commercial LCPs is the poor control over molecular weight and viscosity, inherent to acidolysis polycondensation. As demonstrated in Chapter 3 and 4, control over the viscosity of these materials is crucial for reprocessability, recyclability for the sustainable use of LCPs, and the development of thermoplastic LCP reinforced composites ${ }^{9,12}$. However, both the molecular and textural relaxation times of LCPs decrease significantly when employing LCPs with lower viscosity. This can reduce the orientation and mechanical performance after processing. Similarly, when using LCPs as reinforcing fillers for the production or recycling of thermoplastic blends, control over the viscosity of both the LCP and the matrix is essential in order to generate highly oriented LCP particles in the thermoplastic matrix after injection molding. To address this limitation, we use a chain extension 
reaction to modify a liquid crystalline prepolymer with the aim of obtaining thermoplastic and strong liquid crystalline polymers with a low processing temperature and high relaxation time, while having control over molecular weight and viscosity.

The starting point is the synthesis of a carboxylic acid terminated liquid crystalline prepolymer (LCPP) with a low molecular weight via a conventional melt-state acidolysis polycondensation (Figure 5.1a). Terephthalic acid is used to create an excess of acid groups in the monomer mixture, allowing control over the molecular weight of the LCPP and ensuring acid functionality of the chain ends. A mixture of five different aromatic monomers was chosen to ensure an irregular backbone, which allows lower synthesis and processing temperatures ${ }^{13-15}$. In a post-modification step, a chain extension reaction is performed in order to couple the chain ends of LCPP to yield LCPs with an increased molecular weight. A bis(2-oxazoline)s derived from isophthalic acid was used in the chain extension reaction (IaOx in Figure 5.1b), as 2-oxazolines are well known for their reactivity towards carboxylic acids, while they are thermally stable and have good compatibility with other groups ${ }^{16-18}$. Furthermore, the high reactivity of bis(2-oxazoline)s with carboxylic acids allows the reaction to be conducted during processing, for example in reactive extrusion (Figure 5.1b). Reactive extrusion is commonly used as a means for fast reactions in polymer chemistry ${ }^{19}$, such as compatibilization ${ }^{20,21}$, modification reactions ${ }^{22}$, or even polymerizations ${ }^{23,24}$.

(a) Synthesis of Liquid Crystalline Prepolymer

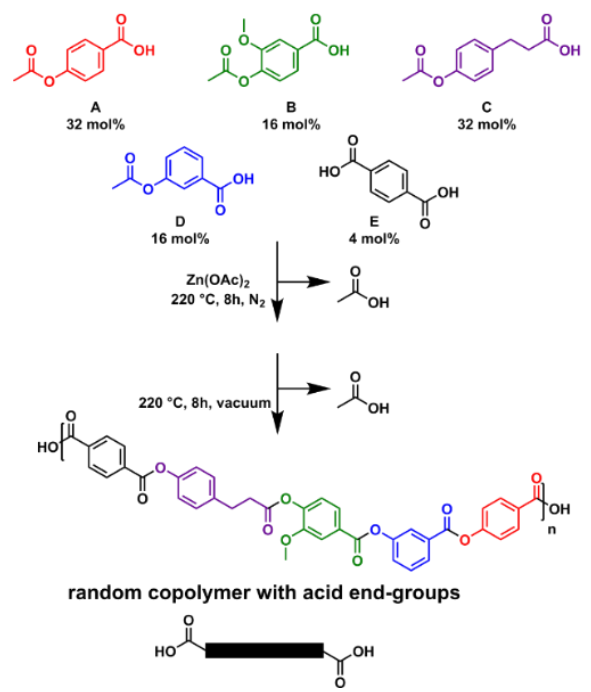

(b) Reactive extrusion of Liquid Crystalline Prepolymer

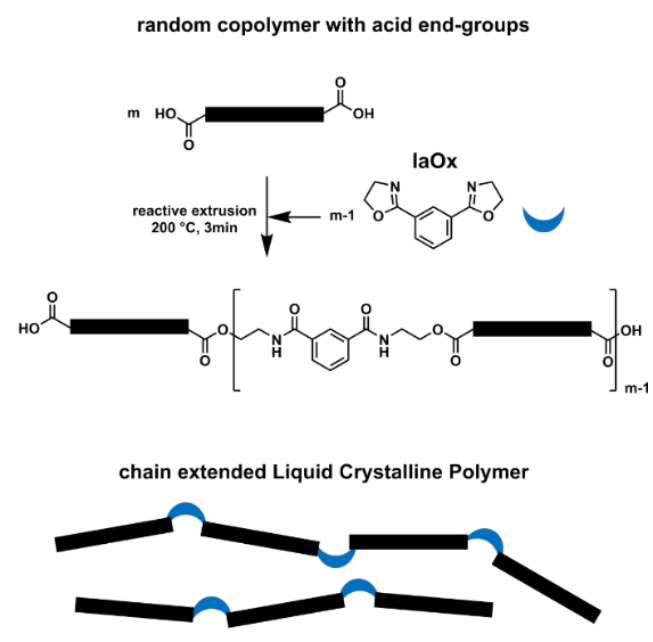

Figure 5.1 (a) Outline of the monomers and conditions used during synthesis of the liquid crystalline prepolymer. (b) Outline of the reagents and conditions used in chain extension of the liquid crystalline prepolymer. 
The molecular weight of the resulting LCP is controlled by stoichiometry between the carboxylic acid and 2-oxazoline moieties during the reactive extrusion step. In addition, the chain architecture can be altered via, for example, the addition of trifunctional carboxylic acid residues. These are key factors with respect to the viscoelastic response of the polymer, and therefore are of great interest in view of the final properties of the material. Interestingly, the chain extended LCPs have a specific backbone structure, consisting of relatively rigid LCPP segments linked via flexible ester-amide moieties (ring-opened $\mathbf{I a O x}$ ) that are formed during reactive extrusion in the nematic melt. This has a pronounced effect on the viscoelastic response of the nematic melt, the relaxation of the nematic texture, and interchain orientation. The comparison of these LCPs with other thermotropic and thermoplastic melts could provide further insight in the properties of the nematic state and the viscoelastic response of polymeric melts.

\subsection{Methods and Materials}

\section{Materials}

4-acetoxybenzoic acid (monomer $\mathbf{A}$ in Figure 5.1a), 4-Hydroxy-3-methoxybenzoic acid, 3-(4-hydroxyphenyl)propanoic acid, 3-hydroxy benzoic acid, 1,3-Bis(4,5-dihydro2-oxazolyl)benzene (IaOx in Figure 5.1b), and 1,3,5-cyclohexanetricarboxylic acid (abbreviated as 3f) were purchased from TCI. Acetic anhydride, terephthalic acid (monomer $\mathbf{E}$ in Figure 5.1a), zinc acetate $\left(\mathrm{Zn}(\mathrm{OAc})_{2}\right)$, and deuterated trifluoroacetic acid ( $d$-TFA) were purchased from Sigma Aldrich. Deuterated chloroform $\left(\mathrm{CDCl}_{3}\right)$ was purchased from Cambridge Isotope Laboratories.

\section{Acetylation Procedure}

4-Hydroxy-3-methoxybenzoic acid (vanillic acid) $(100 \mathrm{~g}, 609.0 \mathrm{mmol})$ was placed in a $1 \mathrm{~L}$ round-bottom flask on a magnetic stirring plate. Dry acetic anhydride $(102 \mathrm{~mL}, 1.0$ mmol) and a catalytic amount of $\mathrm{H}_{2} \mathrm{SO}_{4}$ were added. This mixture was heated to $80{ }^{\circ} \mathrm{C}$ and left to react for $6 \mathrm{~h}$. After cooling the mixture to $0^{\circ} \mathrm{C}, 400 \mathrm{~mL}$ of water was added, and the obtained yellow crystals were obtained via filtration, washed with water, and dried in vacuo at $60{ }^{\circ} \mathrm{C}$ overnight. The obtained crystals were 4-acetoxy-3methoxybenzoic acid (monomer $\mathbf{B}$ in Figure 5.1a), as shown by ${ }^{1} \mathrm{H}-\mathrm{NMR}$ (Figure S5.3b, shown in the Supporting Information).

3-(4-hydroxyphenyl)propanoic acid (phloretic acid) was acetylated via the same procedure. The product obtained via this acetylation was 3-(4-acetoxyphenyl)propanoic acid (monomer $\mathbf{C}$ in Figure 1a), as shown ${ }^{1} \mathrm{H}-\mathrm{NMR}$ (Figure S5.3c, shown in the Supporting Information). 
3-hydroxy benzoic acid was acetylated via the same procedure. The product obtained via this acetylation was 3-acetoxybenzoic acid (monomer $\mathbf{D}$ in Figure 5.1a), as shown ${ }^{1} \mathrm{H}-\mathrm{NMR}$ (Figure S5.3d, shown in the Supporting Information).

\section{Polymerization Procedure}

The polymerization was performed in a $1 \mathrm{~L}$ glass vessel, equipped with a mechanical stirrer. The monomer mixture composed of 4-acetoxybenzoic acid (monomer A, 135.1 g, $750 \mathrm{mmol}$ ), 4-acetoxy-3-methoxybenzoic acid (monomer B, $78.8 \mathrm{~g}, 375 \mathrm{mmol}$ ), 3-(4acetoxyphenyl)propanoic acid (monomer $\mathbf{C}, 156.2 \mathrm{~g}, 750 \mathrm{mmol}$ ), 3-acetoxybenzoic acid (monomer D, $67.6 \mathrm{~g}, 375 \mathrm{mmol}$ ), and terephthalic acid (monomer E, $14.5 \mathrm{~g}, 87 \mathrm{mmol}$ ) was loaded in the glass vessel together with $0.6 \mathrm{~g}$ of zinc acetate. All reagents were dried overnight (in vacuo, $50^{\circ} \mathrm{C}$ ) to remove moisture. The vessel was evacuated and flushed with nitrogen three times prior to the start of the reaction to eliminate oxygen. A small nitrogen flow was applied, the mixture was gradually heated to $220{ }^{\circ} \mathrm{C}$ and the polymerization was allowed to proceed for 8 hours. Next, the nitrogen flow was stopped and reduced pressure was gradually applied ( $<10 \mathrm{mbar}$ ), after which the reaction was allowed to proceed for 8 more hours. The resulting liquid crystalline prepolymer (LCPP) was poured from the hot vessel and used as obtained.

\section{Reactive extrusion and processing}

The prepolymer (LCPP), 1,3-Bis(4,5-dihydro-2-oxazolyl)benzene (IaOx), and 1,3,5cyclohexanetricarboxylic acid (3f) were dried overnight (in vacuo, $50{ }^{\circ} \mathrm{C}$ ) prior to use. Mixtures with the required composition were prepared, where the ratio of LCPP : IaOx reflects the name: For example, the chain-extended polymer CE-2:1 contains $4.48 \mathrm{~g}$ $(1.12 \mathrm{mmol})$ of LCPP and $0.12 \mathrm{~g}(0.556 \mathrm{mmol})$ of IaOx. Next, these mixtures were fed in a preheated DSM Xplore twin-screw micro-extruder with a barrel size of $5 \mathrm{~mL}$ for the reactive extrusion. A recycle channel allows control over the residence time, whereas a valve can be switched to expel the material from the barrel. The materials were mixed for 3 minutes at $200{ }^{\circ} \mathrm{C}$ and $100 \mathrm{rpm}$. After reactive extrusion, the samples were either processed directly into melt-drawn tapes or transferred into a preheated barrel and injection molded into tensile bars. Melt-drawn tapes were prepared using a slit die mounted at the extruder outlet $(0.5 \times 3 \mathrm{~mm}$, produced by DSM Xplore) and a winder (Dienes SD-type). The tapes were drawn and cooled in air. A DSM Xplore IM 5.5 micro injection molder was used to produce tensile bars $(2 \mathrm{~mm} \times 4 \mathrm{~mm} \times 70 \mathrm{~mm}$, with a gage length of $25 \mathrm{~mm}$ ). The barrel temperature was set to $180^{\circ} \mathrm{C}$, while the mold temperature was set to $25{ }^{\circ} \mathrm{C}$. An injection pressure of 8 bar was used for all samples. Injection molded bars and melt-drawn tapes of pure LCPP were produced under the same 
processing conditions as the chain extended materials as reference (with the exception that no $\mathbf{I a O} \mathbf{x}$ was added).

\section{Material Characterization}

The chemical composition of the monomers and LCPS were evaluated via ${ }^{1} \mathrm{H}-\mathrm{NMR}$ analysis. A Bruker Ultrashield 300 spectrometer (300 MHz magnetic field) was used to record ${ }^{1} \mathrm{H}-\mathrm{NMR}$ spectra. The samples were dissolved in a mixture of $\mathrm{CDCl}_{3}$ and $d$-TFA (both solvents were mixed in approximately equal volumes).

The molecular weight distributions of the LCPP and the chain extended LCPs were determined via gel permeation chromatography (GPC) using a PSS SECcurity GPC system with Agilent 1260 Infinity instrument technology. The system is equipped with two PFG combination medium micro-columns with $7 \mu \mathrm{m}$ particle size $(4.6 \times 250 \mathrm{~mm}$, separation range 100-1.000.000 Da), a PFG combination medium pre-column with 7 $\mu \mathrm{m}$ particle size $(4.6 \times 30 \mathrm{~mm})$, and a Refractive Index detector $(\mathrm{RI})$. Distilled 1,1,1,3,3,3hexafluoroisopropanol (HFIP) containing $0.019 \%$ sodium trifluoroacetate was used as mobile phase at $40{ }^{\circ} \mathrm{C}$, with a $0.3 \mathrm{~mL} \mathrm{~min}^{-1}$ flow rate. The obtained molecular weight distributions are relative with respect to poly(methyl methacrylate) standards obtained from PSS. Samples were prepared via dissolution of approximately $6 \mathrm{mg}$ of LCP in 1.5 $\mathrm{ml}$ HFIP. The samples were shaken overnight and subsequently filtered over a $0.2 \mu \mathrm{m}$ PTFE syringe filter prior to injection.

The viscoelastic behavior of the pure materials was determined in a MCR 702 TwinDrive rheometer (Anton Paar) with a parallel plate geometry (diameter of $12 \mathrm{~mm}$, gap of $0.7 \mathrm{~mm}$ ). Samples were loaded at $190^{\circ} \mathrm{C}$, kept isothermal for 10 minutes to erase mechanical and thermal history, cooled to $150{ }^{\circ} \mathrm{C}$ and subjected to a frequency sweep with a strain of $1 \%$.

The glass transition temperature $\left(T_{\mathrm{g}}\right)$ was determined by differential scanning calorimetry (DSC) using a TA Instruments Q2000 DSC. The heating and cooling-rates of the sample were $10{ }^{\circ} \mathrm{C} \mathrm{min}-1$ and measurements were performed under a nitrogen rich atmosphere.

The nematic texture of the LCPs was evaluated via polarized optical microscopy (POM) using an Olympus BX53 Microscope (50 times magnification) equipped with an Olympus DP26 camera and a Linkam HFSX350 temperature controlled stage. The samples were heated to $200{ }^{\circ} \mathrm{C}$ between two glass plates and then pressure was applied to decrease the sample thickness. Subsequently the samples were cooled to $180{ }^{\circ} \mathrm{C}$ and annealed for 15 minutes. The relaxation of the nematic texture was studied on microtomed slices (thickness $\sim 20 \mu \mathrm{m}$, using a Reichert-Jung 2040) of the respective 
melt-drawn tapes. The slices were placed between two glass plates and heated at a rate of $30{ }^{\circ} \mathrm{C} \mathrm{min}^{-1}$ to $180{ }^{\circ} \mathrm{C}$,

Wide-angle X-ray Diffraction (WAXD) measurements (wave length, $\lambda=0.104 \mathrm{~nm}$ ) were carried out at the European Synchrotron Radiation Facility (ESRF), the DUBBLE beamline (BM26B, Grenoble, France). The DUBBLE beamline is optimized for polymer science as is reported by Bras et al. ${ }^{25}$ and Portale et al. ${ }^{26}$. WAXD patterns, with an exposure time of $5 \mathrm{~s}$, were collected using a Frelon detector $(2048 \times 2048$ pixels of $48.8 \mu \mathrm{m} \times 48.8 \mu \mathrm{m}$ ) placed at a distance of $0.18 \mathrm{~m}$. The data were normalized for synchrotron beam fluctuations using an ionization chamber placed before the sample. Furthermore, a correction for the sample absorption was performed using a photodiode located at the beamstop before the background contribution was subtracted. The wavenumber $q=4 \pi \sin \theta / \lambda$, with $\theta$ being half of the scattering angle for WAXD experiments scale calibration, has been achieved by $\alpha-\mathrm{Al}_{2} \mathrm{O}_{3}$ (alumina). The samples were loaded into a Linkam CSS 450 shear cell, of which the glass windows were substituted by polyimide film to ensure low background scattering from the device. The shear cell was used to study the relaxation of the interchain orientation of the LCPs. A stack of melt drawn tapes was wrapped in polyimide-tape $\left(\right.$ kapton $\left.{ }^{\circledR}\right)$. The stack of meltdrawn tapes was heated to $180{ }^{\circ} \mathrm{C}$ at a rate of $30^{\circ} \mathrm{C} \mathrm{min}-1$, followed by an isothermal period of 10 minutes. The orientation parameter $(\mathrm{S}),\left\langle\mathrm{P}_{2 \mathrm{n}}(\cos \varphi)\right\rangle_{\mathrm{d}}$, was calculated from the obtained diffraction patterns via a procedure by Mitchell and Windle ${ }^{27}$, as described in Chapter 2.

The mechanical properties of the injection molded LCPs were assessed under tension. Measurement were performed on a Zwick Z100. Samples were subjected to a constant deformation rate of $5 \mathrm{~mm} \mathrm{~min}^{-1}$, at room temperature.

\subsection{Results and Discussion}

\section{Polymer Synthesis and chemical analysis}

As addressed in the introduction, there is potential for versatile thermotropic LCPs with low processing temperatures from an application and sustainability point of view. A decrease in the processing and synthesis temperatures prevents thermal degradation. This plays a key role in increasing the reprocessability of the LCP itself, the development of reprocessable/recyclable composites, and successful incorporation of the thermally labile (renewable) monomers. In addition to the chemical constituents, the mechanical properties of the LCPs are known to be dependent on their molecular orientation, a parameter that is mostly governed by the processing conditions and the LCP viscosity. Therefore, to obtain improved control over the viscosity and molecular weight of the LCPs developed in this study (compared to regular acidolysis polycondensation), we 
build up molecular weight during reaction extrusion of a low molecular weight thermotropic prepolymer LCPP and a bis(2-oxazoline) at mild temperatures (Figure 5.1).

For the synthesis of the LCPP, a mixture of 4-acetoxybenzoic acid (A), 4-acetoxy-3methoxybenzoic acid (B), 3-(4-acetoxyphenyl)propanoic acid (C), and 3-acetoxybenzoic acid $(\mathbf{D})$ is used to yield an amorphous, liquid crystalline polymer. Additionally, the use of terephthalic acid (E) introduces an excess of carboxylic acid functional groups compared to the acetoxy esters and provides the means to control the molecular weight while ensuring that all end-groups are carboxylic acids and thus reactive during the chain-extension step (Figure 5.1a). The targeted molecular weight for the LCPP is 4.0 $\mathrm{kg}$ mol-1, with the aim to ensure that a liquid crystalline polymer is obtained having sufficient concentration of carboxylic acid end groups for chain extension. The use of five co-monomers in melt acidolysis polycondensation allows a reduced synthesis temperature $\left(220^{\circ} \mathrm{C}\right)$ of the $\mathbf{L C P P}$ as the resulting irregularity of the polymer chains prevents their crystallization, ensuring that the LCPs can readily be processed at $200{ }^{\circ} \mathrm{C}$ and lower. Though not the focus in this work, we want to highlight that $52 \%$ (by weight) of the resulting LCPP can be obtained from renewable feedstock, since the monomers $\mathbf{B}$ and $\mathbf{C}$ can be produced from biomass ${ }^{11,28}$.

The chemical composition of the obtained LCPP is evaluated via ${ }^{1} \mathrm{H}-\mathrm{NMR}$ analysis, as shown in Figure 5.2 (bottom). An absence of signals in the $2.4-2.5 \mathrm{ppm}$ range indicates the absence of acetoxy-groups, confirming that the acidolysis polycondenstation reached a high conversion. The presence of doublet around 3.18 ppm arises from the $\mathrm{CH}_{2}$ groups of monomer $\mathbf{C}$ present in the polymer backbone, whereas the shoulder at $2.86 \mathrm{ppm}$ corresponds to the $\mathrm{CH}_{2}$ groups of monomer $\mathbf{C}$ located at the chain end (i.e. at the terminal carboxylic acid group). The singlet at 4.02 $\mathrm{ppm}$ corresponds to the methoxy-group of monomer B. Note, due to significant overlap between the numerous aromatic signals and the signal corresponding to the solvent (chloroform, $7.28 \mathrm{ppm}$ ), we could not determine the ratio between the incorporated monomers $\mathbf{A}, \mathbf{D}$, and $\mathbf{E}$. Nevertheless, the observed ratio of the integrals of the signals in the aliphatic region to those in the aromatic region $(1: 2.18)$, is very close to the expected value based on the ratio in the monomer feed $(1: 2.15)$. Overall, this suggests that the observed LCPP composition is close to the targeted composition (Table S5.1, Supporting Information). 


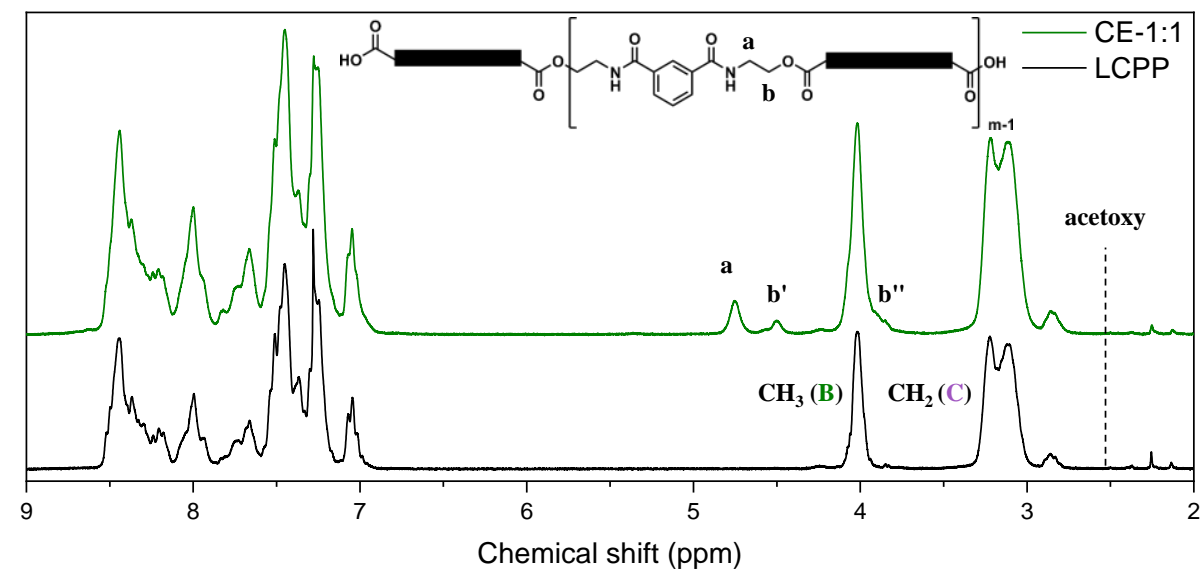

Figure 5.2 ${ }^{1} H$-NMR traces of the prepolymer (LCPP, bottom) and the chain extended polymer (CE-1:1, top). Both spectra are measured in a mixture of $C D C l_{3} / d-T F A$.

The LCPP is subjected to a chain-extension reaction with 1,3-bis(4,5-dihydro-2oxazolyl)benzene (IaOx) in the melt where the 2-oxazoline moieties and the terminal carboxylic acids of LCPP react into ester-amide bonds via a thermal ring-opening addition reaction (Figure 5.1b). Considering that the reaction time required for this process is in the order of seconds to minutes at the processing temperatures of $200{ }^{\circ} \mathrm{C}^{18,29}$, the reaction is conducted during extrusion at $200{ }^{\circ} \mathrm{C}$ for three minutes. Additionally, via control of the molar ratio of bis(2-oxazoline), introduced during extrusion, LCPs with targeted molecular weight can be synthesized (Table 5.1) providing control over the melt-viscosity and, hence, the relaxation time. Note, the names of the samples reflect the ratio of LCPP : IaOx, being 2:1 (CE-2:1), 3:2 (CE3:2) and 1:1 (CE-1:1). In addition, a sample is prepared where the tri-functional 1,3,5cyclohexanetricarboxylic acid (3f) is added to induce branching (CE-3f). Figure 5.2 (top) shows a characteristic ${ }^{1} \mathrm{H}-\mathrm{NMR}$ spectrum of the chain extended polymer CE-1:1. Generally, we do not observe any signal corresponding to the unreacted IaOx (See Supporting Information for the NMR spectra of the unreacted IaOx, Figure S5.2), as we only observe signals $\mathbf{a}$ and $\mathbf{b}$, corresponding to the methylene protons of the esteramide moiety generated after the ring opening addition reaction of the 2-oxazoline ring with a carboxylic acid. Note, the signal corresponding the methylene next to the ester groups (signals b, Figure 5.2) split up and are located at $4.5 \mathrm{ppm}$ (b') and $3.8 \mathrm{ppm}(\mathrm{b}$ '), which are expected to correspond to the ester bonds connected to aromatic and aliphatic spacers, respectively. Nevertheless, within the experimental error of the NMR analysis and in line with literature findings, we observe that the chain extension proceeds quantitatively within the selected reactive extrusion conditions. 
Table 5.1 Molar ratio, calculated molecular weight $\left(M_{n}\right)$, molecular weight $\left(M_{w}\right)$ and dispersity (Ð) (as measured by GPC) for the prepared samples. Note that 1,3,5-cyclohexanetricarboxylic acid (3f), was added to $\boldsymbol{C E}-\mathbf{3 f}$, to induce branching.

\begin{tabular}{|c|c|c|c|c|}
\hline Material & $\begin{array}{c}\text { Molar ratio } \\
(\text { LCPP }: \text { IaOx }: 3 \mathrm{f})\end{array}$ & $\begin{array}{c}M_{\mathrm{n}} \text { calculated } \\
\left(\mathrm{kg} \mathrm{mol}^{-1}\right)\end{array}$ & $\begin{array}{c}M_{\mathrm{w}} \\
\left(\mathrm{kg} \mathrm{mol}^{-1}\right)\end{array}$ & $\begin{array}{c}\text { PDI } \\
(-)\end{array}$ \\
\hline LCPP & $1: 0: 0$ & 4.0 & 6.3 & 6.0 \\
\hline CE-2:1 & $2: 1: 0$ & 8.0 & 15.3 & 9.7 \\
\hline CE-3:2 & $3: 2: 0$ & 12.0 & 20.2 & 11.7 \\
\hline CE-1:1 & $1: 1: 0$ & $-^{\mathrm{a}}$ & 40.5 & 15.6 \\
\hline CE-3f & $0.95: 1: 0.05$ & $-\mathrm{b}$ & 77.3 & 25.1 \\
\hline
\end{tabular}

${ }^{a}$ The calculated $M_{n}$ is infinite given that the carboxylic acids and 2-oxazoline moieties are present in perfect stoichiometry and fully react without the formation of cyclic polymeric structures.

${ }^{b}$ The tri-functional carboxylic acid (3f) induces branching, and therefore no target $M_{n}$ is given.

(a)

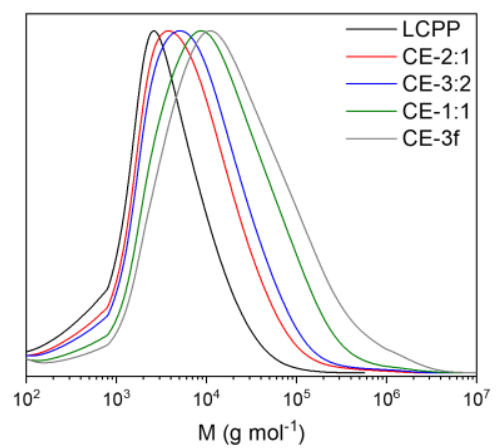

(b)

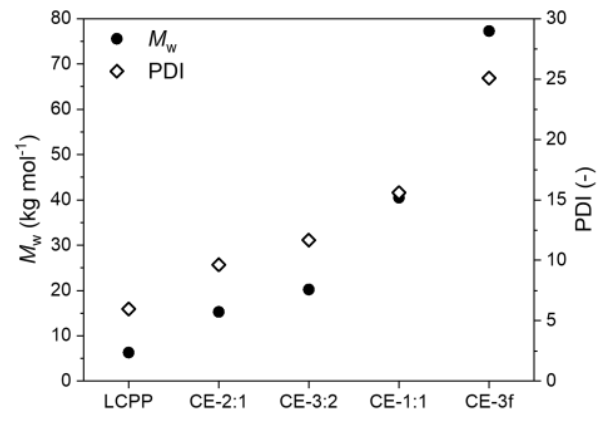

Figure 5.3 (a) Molar mass distributions of LCPP and chain extended LCPs after (reactive) extrusion. (b) Weight average molar mass and PDI of LCPP and chain extended LCPs.

The changes in molecular weight and dispersity, after the chain extension, are evaluated via GPC analysis. The resulting molar mass distributions (Figure 5.3a) show a clear increase in molecular weight (Table 5.1), with an increasing ratio of LCPP : IaOx. The molar mass distribution of CE-1:1 has a small shoulder on the high molar mass side, indicative of the presence of branched chains. This is likely the result of a side reaction where a 2-oxazoline moiety reacts with the amide formed upon chain extension, forming a branch point ${ }^{18}$. As expected, this shoulder is considerably more pronounced in polymer $\mathbf{C E - 3 f}$, confirming that the use of trifunctional carboxylic acids during extrusion allows modification of the chain architecture via branching. An overview of the molecular weight and PDI of the obtained LCPs is displayed in Figure 5.3b. Within experimental error, the increase in $M_{\mathrm{w}}$ scales with the employed LCPP : IaOx ratio, highlighting the effectiveness of the reactive extrusion step to control the molecular weight of the polymer. Additionally, this observation indicates the validity of the 
estimated molar mass of LCPP and the assumption of LCPP being carboxylic acid terminated.

\section{Thermal and viscoelastic behavior}

The thermal behavior of the synthesized polymers is evaluated via DSC (Figure 5.4a). In general a rather broad glass transition $\left(T_{\mathrm{g}}\right)$ with an onset between 70 and $80{ }^{\circ} \mathrm{C}$, is observed. The $T_{\mathrm{g}}$ of these materials is determined as the first tangental intersection within this broad transition (demarked by the black lines). For all polymers, this glass transition is followed by a broad signal, possibly originating from the continuation of the increase in segmental mobility in combination with the potential melting of a minor and diverse population of irregular non-periodic layer (NPL) crystallites ${ }^{11,30}$. Nevertheless, there is a clear increase in $T_{\mathrm{g}}$ as the molecular weight of the polymers increases. This trend is commonly observed in polymers with low molecular weight and generally levels off upon reaching a critical molecular weight ${ }^{31}$.

(a)

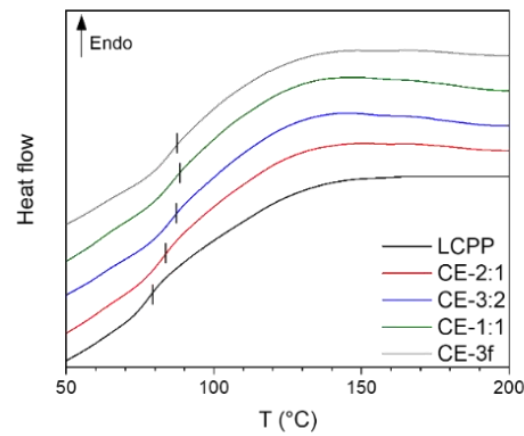

(c)

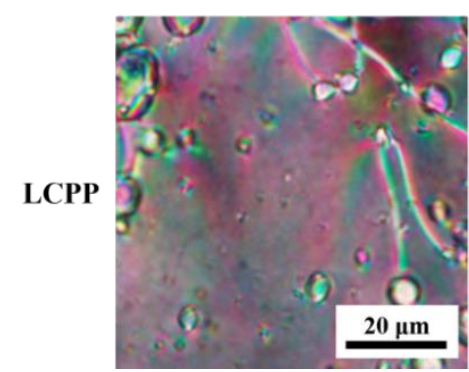

(b)

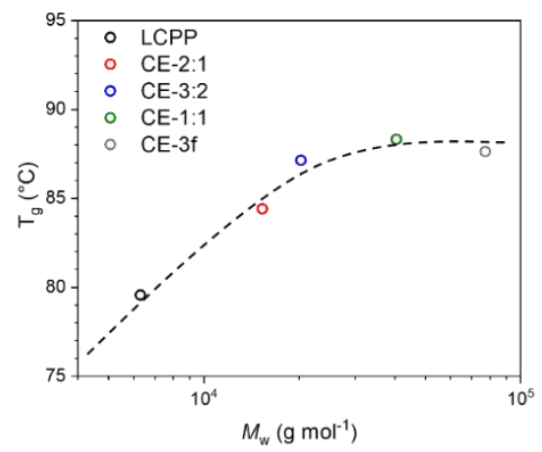

CE-1:1

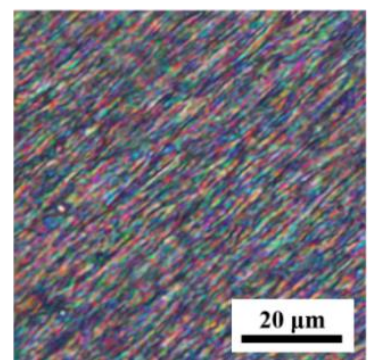

Figure 5.4 (a) Second DSC heating trace the polymers developed in this study, measured at a heating rate of $10{ }^{\circ} \mathrm{C} \mathrm{min}$. (b) Dependence of the glass transition temperature on the weight average molecular weight of the LCPs. Note, a dotted line is provided to guide the eye. (c) Schlieren textures of LCPP and CE-1:1, viewed between crossed polarizers after 15 minutes of annealing at $180^{\circ} \mathrm{C}$.

The same trend is also observed in the LCPs developed in this study, as is highlighted in Figure 5.4b. Despite the potential presence of a low fraction of NPL crystallites based 
on the DSC traces, the LCPP and the LCPs after chain extension are considered to be amorphous. This is further confirmed by the typical nematic Schlieren texture observed in polarized optical microscopy (POM) at $180^{\circ} \mathrm{C}$, as is depicted for LCPP and CE-1:1 (Figure 5.4c). Additionally, the amorphous nature is confirmed by the absence of distinct diffraction peaks in the Wide-angle X-ray Diffraction pattern of the samples, which will be discussed in a later section. All synthesized LCPs can be considered nematic glasses, since no crystallization is observed upon cooling or annealing. The LCPs exhibit a nematic phase above their respective $T_{\mathrm{g}}$ and the nematic ordering persists upon cooling below $T_{\mathrm{g}}$. The nematic to isotropic transition could not be observed due to thermal degradation prior to the transition.

To identify the effect of the chain-extension reaction on the viscoelastic response, experiments in the linear viscoelastic region are performed using a rheometer equipped with parallel plates. To remove the sample history, the samples are kept under isothermal conditions at $190^{\circ} \mathrm{C}$ for 10 minutes and subsequently cooled to $150^{\circ} \mathrm{C}$ after which frequency sweep experiments are performed isothermally in the linear viscoelastic regime (Figure 5.5a). Deviating from typical behavior of flexible polymers, the viscoelastic behavior of LCPs is found to be rather complex, which is attributed to the superimposed contribution of chain dynamics and the processes related to the short range order in the nematic melt ${ }^{32-34}$. Nevertheless, we clearly observe an increase in the complex viscosity with the molecular weight though a Newtonian plateau is not observed for the chain-extended LCPs. The frequency dependence of the viscous modulus $\left(G^{\prime \prime}\right)$ and the elastic modulus $\left(G^{\prime}\right)$ of the respective LCPs is displayed in Figure 5.5b. As expected, LCPP displays the typical behavior of nematic thermotropic polymers, with relatively viscous behavior at higher frequencies, and a strong structural contribution at lower frequencies (indicated by the similar slopes of G' and G' below $\omega \sim 10 \mathrm{rad} \mathrm{s}^{-1}$ ). With an increase in molecular weight, the respective moduli increase and the melt response becomes more elastic, which is indicated by the more drastic increase in G' compared to G". In contrast to LCPP, which displays a slope for G" of 1 over the evaluated frequency range, the slope of G" for CE-2:1 approaches 0.5, normally corresponding to Rouse dynamics ${ }^{35}$. Furthermore, the LCPs with higher molecular weights (CE-3:2 and CE-1:1) show a fundamental shift in the state of the melt: a frequency range exists where the elastic modulus $\left(G^{\prime}\right)$ surpasses the viscous modulus $\left(G^{\prime \prime}\right)$ and a crossover point is observed. Combined with the fact that the slope of the respective moduli decrease below 0.5 , this is a clear indication of network behavior, which is charactestically observed in entangled polymer melts. The crossover points shift to lower frequencies with an increase in the molecular weight. For example, the crossover for $\mathbf{C E}-1: 1$ occurs at a lower frequency $\left(\sim 0.25 \mathrm{rad} \mathrm{s}^{-1}\right)$ compared to $\mathbf{C E}$ - 
3:2 $\left(\sim 4.3 \mathrm{rad} \mathrm{s}^{-1}\right)$. In the case of $\mathbf{C E}-3 \mathbf{f}$, the elastic modulus exceeds the viscous modulus over the measured frequency range, and a crossover is not observed (shown in Figure S5.4b of the Supporting Information). This is likely the result of the branched structure of polymer CE-3f, resulting from the addition of a tri-functional carboxylic acid during reactive extrusion. To summarize, with increasing molecular weight we observe the transition from an unentangled nematic melt to a nematic melt where a physical network appears to be present. The presence of a physical network in the nematic melt can be beneficial in achieving a higher degree of interchain orientation in a melt spinning process, which is a prerequisite for the development of fibers with a high tensile modulus and a high tensile strength ${ }^{36}$.

(a)

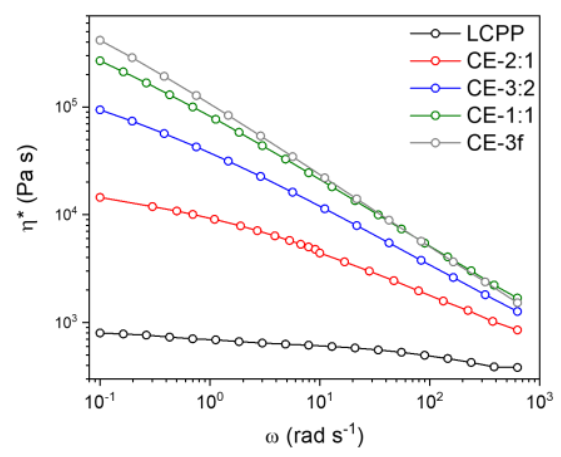

(b)

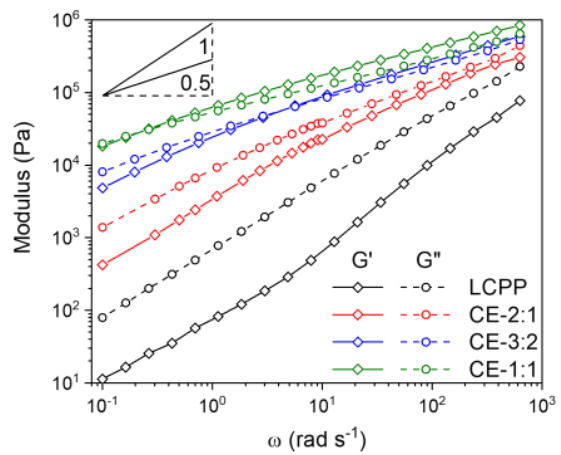

Figure 5.5 (a) Complex viscosity as function of the angular frequency ( $\omega)$ of LCPP and chain extended LCPs, measured at $150^{\circ} \mathrm{C}$. (b) Storage and loss modulus as function of angular frequency of LCPP and chain extended LCPs, measured at $150^{\circ} \mathrm{C}$ in the linear viscoelastic regime. The lines in the top left corner mark slopes of 1 and 0.5 , respectively.

The viscoelastic behavior of CE-3:2 and CE-1:1 resemble that of an entangled flexible polymer melt, as a physical network is observed at high frequencies, followed by a crossover point at a lower frequency. However, we consider it unlikely that these rigid thermotropic LCPs are constrained in the same fashion as typical flexible polymers. In flexible thermoplastic polymers the constraints are due to entanglements caused by the overlapping of chains in a random coil conformation (Figure 5.6) ${ }^{37}$. Even though there are numerous methylene spacers present in the LCPs (from $\mathbf{I a O x}$ and monomer $\mathbf{C}$ ), which decreases the rigidity of the backbone, the nematic nature of the LCPs implies that the persistence length remains high. The considerable rigidity of the LCP backbone in combination with the relatively low molecular weight of the LCPs (in the order of 5 $\left.-50 \mathrm{~kg} \mathrm{~mol}^{-1}\right)$ makes it unlikely for the LCP chains to adopt a random coil conformation and to be constrained by entanglements in the classical sense. Nevertheless, the presence of a physical network remains clear based on the rheological data. 

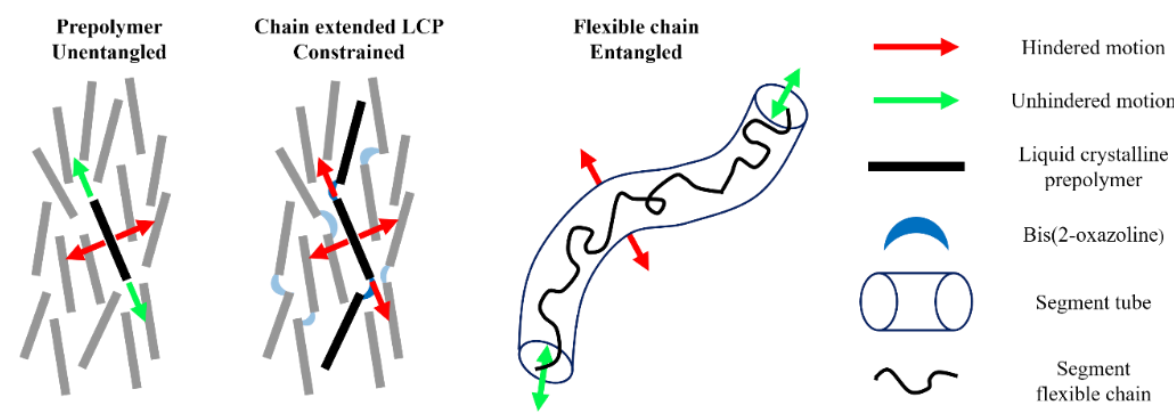

Figure 5.6 Scheme representing the mobility of polymer chains or chain segments with respect to their axis. From left to right: a typical nematic melt (representative LCPP), a constrained nematic melt (representative chain extended LCPS), and an entangled melt (representative for flexible polymers).

Instead, we consider it more likely that constraints arise from the fact that the chain extension reaction, where different $\mathbf{L C P P}$ chains are coupled via $\mathbf{I a O x}$, was conducted in the nematic melt. The LCPP chains locally share a common orientation direction in the nematic melt, while long-range and positional order are absent. As a result, the uncoupled LCPP chains have a high degree of mobility along the direction of the chains, whereas motion perpendicular to the chain direction is limited (Figure 5.6, left). As the chain ends of the $\mathbf{L C P P}$ chains are coupled during reactive extrusion with $\mathbf{I a O} \mathbf{X}$ in CE-3:2 and CE-1:1, the mobility of the chains along their respective chain direction is drastically impaired. For example, motion in the chain direction would now require the movement coupled LCPP segments, which do not necessarily share the same orientation direction (Figure 5.6, second image from left). It is likely that the coupled chains adopt an extended conformation due to the complex flow field that is present during reactive extrusion, although this conformation is unlikely a requirement for the chain extension reaction itself. Accordingly, we do not consider that the folded conformation of the flexible $\mathbf{I a O} \mathbf{x}$ linkers provides the constraints responsible for the network behavior, which is in line with the low average number of linkages required per chain (only two, in the case of CE-3:2) to introduce network behavior that persists over multiple decades in terms of timescale. As the average number of LCPP chains that are coupled increases with the amount of $\mathbf{I a O x}$, the LCP chains are severely constrained, resulting in the physical network that is observed in CE-3:2 and CE-1:1. This implies that, since the nature of the network differs from an entangled melt, the relaxation mechanism is likely to deviate from reptation, and will likely require some level of cooperative motion. 
(a)

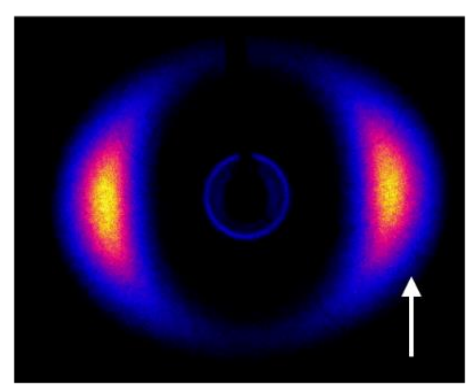

(c)

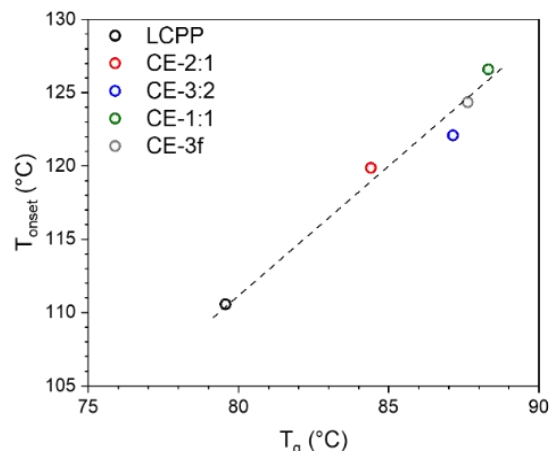

(b)

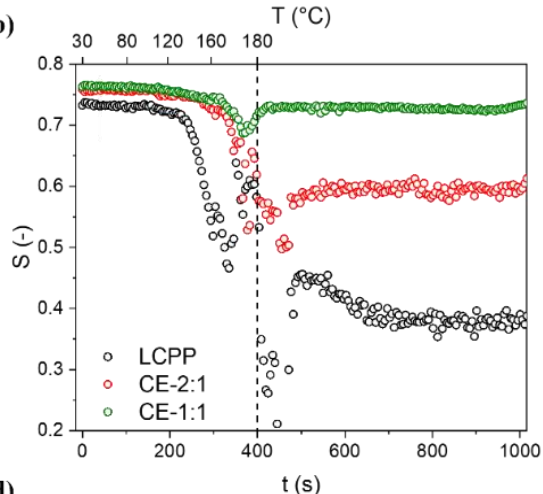

(d)

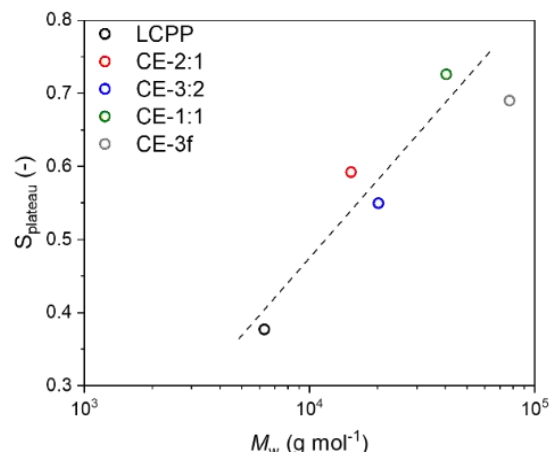

Figure 5.7 (a) X-ray diffractrogram of an injection molded LCPP bar, the arrow marks the flow direction. (b) Relaxation of the orientation parameter (S) of LCP tapes wrapped in polyimide tape as recorded upon heating. Beyond $400 \mathrm{~s}$ the sample is kept under the isothermal condition $180^{\circ} \mathrm{C}$. (c) Onset temperature of relaxation as a function of the glass transition temperature of the respective LCPs. (d) Final obtained degree of orientation ( $\left.S_{\text {platean }}\right)$ for the heated LCP materials as a function of their respective molecular weights.

To investigate the effect of molecular weight and the concomitant changes in the viscoelastic behavior on the microstructure and interchain orientation of the respective LCPs, Wide-angle X-ray Diffraction (WAXD) is employed (Figure 5.7a for LCPP). The broad diffraction signal is characteristic for an amorphous polymer and the azimuthal arcs indicate a significant level of interchain orientation. Note that the ring at low diffraction angles, close to the beam stop, corresponds to a signal from the polyimidetape used to hold the sample in place. In order to study the relaxation of the interchain orientation of the produced LCPs, stacks of the melt-drawn tapes (width $\sim 1 \mathrm{~mm}$, thickness $\sim 0.16 \mathrm{~mm}$ ) are wrapped in a polyimide-tape and heated while collecting data at the synchrotron source. The orientation parameter $(S)$ is monitored for the samples while heating (Figure 5.7b). At the start of the experiment, the orientation parameter of all the LCP tapes is similar ( $\mathrm{S} \sim 0.76$ ), except for LCPP, which exhibited a slightly lower degree of orientation $(S \sim 0.73)$. As the samples are heated above their respective $T_{\mathrm{g}}$, 
the increased mobility of the polymer chains results into a slight decrease of the orientation parameter with temperature ( $\left.T_{\text {onset }}\right)$. Upon further heating, the mobility of the LCPs increases and the tapes soften. This simultaneously causes further relaxation of chains and fusion of the stacked tapes. For the chain extended LCPs, the relaxation and the fusion of tapes, occur at higher temperatures (above $160^{\circ} \mathrm{C}$ ) compared to the prepolymer, LCPP $\left(130{ }^{\circ} \mathrm{C}\right)$. In this process the orientation parameter decreases rapidly, which is partly caused by the fusion of the tapes. After fusion of the tapes, the orientation parameter recovers leading to the observation of a minimum. Thus, the minimum observed in the orientation parameter is an artifact originating from the fusion of the tapes. The orientation parameter finally reaches a constant value $\left(S_{\text {plateau }}\right)$, which represents the molecular orientation above the glass transition temperature and shows dependence on the molar mass of the LCP. A clear trend emerges from the relaxation behavior; the onset of relaxation is delayed and the material retains a higher degree of orientation with the increasing molecular weight, to the point that there is hardly a decrease in orientation upon heating, as is the case for CE-1:1.

The onset of interchain relaxation, as evaluated by $T_{\text {onset, }}$, correlates strongly with the onset of chain mobility (corresponding to $T_{\mathrm{g}}$ ) as is shown in Figure 5.7c. Since cooperative mobility is the prerequisite for the system to relax its dependence on $T_{\mathrm{g}}$ is apparent. However, it implies that the molecular weight can be a factor governing the onset of relaxation. Especially at low molecular weight, the $M_{\mathrm{w}}$ influences the $T_{\mathrm{g}}$, indicating that a higher molecular weight or the presence of branched chains can be utilized effectively to postpone relaxation to some extent. More interesting is the correlation between the equilibrium orientation parameter ( $\left.S_{\text {plateau }}\right)$ and the $M_{\mathrm{w}}$ of the respective LCPs (Figure 5.7d). As the molecular weight of the LCPs increases so does $S_{\text {plateau, }}$ which means that the LCPs that were chain extended via reactive extrusion with IaOx retain a significant degree of interchain orientation, even after being kept at approximately $100{ }^{\circ} \mathrm{C}$ above their $T_{g}$ for 10 minutes. This observation in itself is remarkable as it implies significant hindrance in the underlying relaxation processes, at least on the cooperative length scale. The apparent lack of relaxation might be caused by the increase of the viscosity of the LCP in combination with the establishment of a physical network state, which would require cooperative motion of multiple LCP segments or chains in order to relax. Due to the coupling of LCPP chains during chain extension, once the network is established it would be nearly impossible for chains to disengage and relax, as previously exemplified in Figure 5.6.

From the previous section, it is clear that the interchain relaxation proceeds significantly slower in LCP materials with increasing molecular weight. Recently we reported on a potential interrelation between the relaxation of the LCP texture and interchain 
orientation $^{38}$. We observed that relaxation of the nematic texture consists of an initial retraction of stretched domains, followed by coalescence of these domains. More importantly, the relaxation of the interchain orientation is mainly driven by the coalescence of the nematic domains. For example, for samples having a domain texture far from equilibrium (i.e. containing very fine nematic domains formed when the material is subjected to high shear rates), coalescence, and correspondingly interchain relaxation, proceeds very rapidly. In contrast, for samples subjected to low shear rates, i.e. having a nematic domain texture close to the equilibrium texture, coalescence of the nematic domains is strongly retarded and limited interchain relaxation is observed. Therefore, to identify whether the retardation in interchain relaxation with increasing molecular weight, as seen in WAXD, can be correlated to the relaxation of the nematic texture, polarized optical microscopy studies were performed (Figure 5.8). To this end, microtomed slices of the melt drawn tapes of LCPP and CE-1:1 are subjected to the same experimental protocol as for the WAXD experiments.

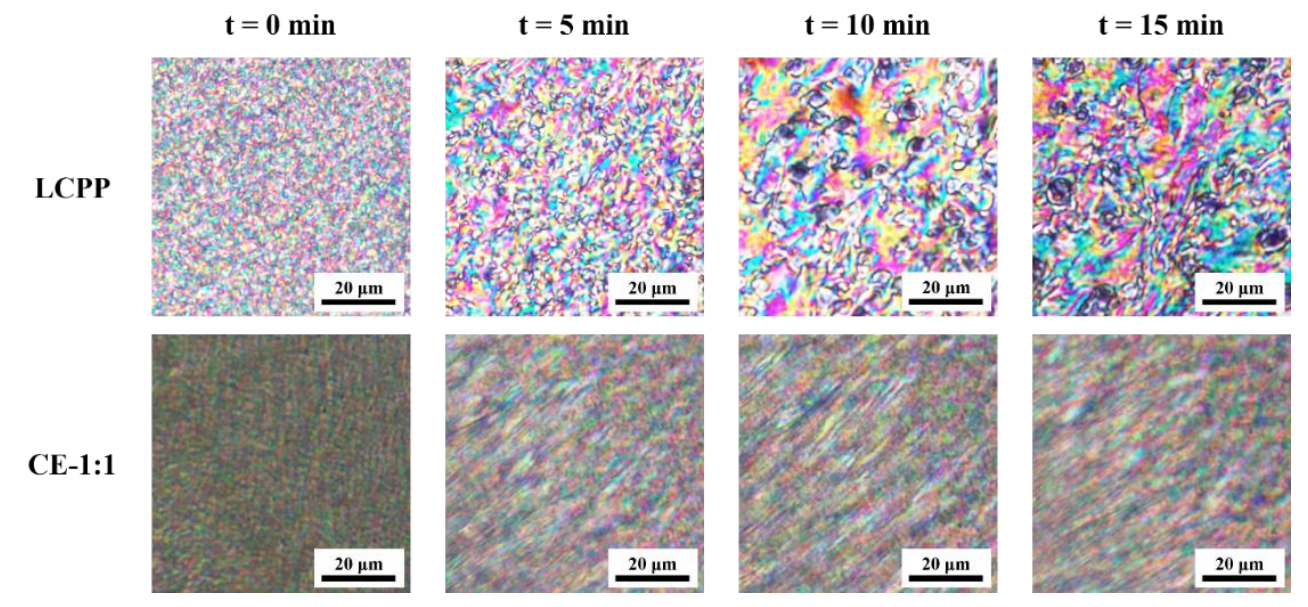

Figure 5.8 Evolution of the nematic texture, observed via POM, upon annealing at $180{ }^{\circ} \mathrm{C}$ of microtomed slices. Images for melt drawn tapes of LCPP (top) and CE-1:1 (bottom) are shown.

In the case of LCPP, the initial retraction of the stretched domains occurred during heating; however, the nematic texture coarsens considerably over the 15 min annealing period. In contrast, compared to LCPP, the molecular weight and viscosity of CE-1:1 are considerably higher, resulting in a drastic change in timescales. Initially, the image brightens, indicating a (partial) retraction of submicron domains; however, on longer timescales no significant change in texture is observed; oriented nematic domains remain present, implying significant inhibition of contraction and coalescence. As outlined above, given that the relaxation of the interchain orientation is mainly driven by the textural coalescence of the nematic domains, the findings from optical 
microscopy indeed suggest CE-1:1 should not undergo extensive interchain relaxation, which is in agreement with the observations from WAXD.

Upon chain extension, the molecular weight and the viscosity increase significantly, explaining in part the slower relaxation of interchain orientation. However, the changes in $S_{\text {plateau }}$ and the textural evolution of the melt upon chain extension do not simply imply a delay of relaxation, but the retention of a significant degree of interchain orientation. The scaling of the relaxation timescale with the LCP viscosity, as observed in Chapter 2 and 3, was not observed for these $\mathrm{LCPs}^{9}, 38$. Therefore, it is likely that there is another factor involved: the physical network formed during the chain extension reaction. The transition from an unentangled nematic melt to a constrained one, as was observed for the chain-extended LCPs (eg. CE-1:1) is likely to play a dominant role, given that this fundamentally changes the chain dynamics and time scales in the nematic melt.

The chain extended LCPs, such as CE-1:1, show a delayed relaxation of the nematic texture and interchain orientation, even after the application of high shear rates, as exemplified by WAXD and POM. This behavior is unusual, considering that fast relaxation is typically observed in thermotropic systems with a fine polydomain texture caused by the application of high deformation rates. To recall, the relaxation of the nematic texture of commercial LCPs having rigid backbone proceeds within several hundred seconds following the application of low to moderate shear rates ${ }^{39-41}$. For commercial thermotropic LCPs it has been established that an increased flexibility of the polymer backbone ${ }^{42}$ and the application of higher shear rates ${ }^{43}$ reduces the relaxation time of the nematic texture to several tens of seconds. Thus the behavior of the chain extended LCPs developed in this study, with relatively flexible backbones, deviates from the commercial thermotropic LCPs having similar molar masses (typically 20 to $\left.40 \mathrm{~kg} \mathrm{~mol}^{-1}\right)$.

A possible reasoning behind the limited relaxation of the chain extended LCPs is that the rearrangement of chains and chain segments required for relaxation are severely hindered in constrained nematic melts, as supported by our observations regarding the relaxation of the interchain orientation and the nematic texture. The changes likely arise from the presence of a physical network on the relaxation of the interchain orientation of LCPs is schematically illustrated in Figure 5.9. In the classical case of unentangled (low molecular weight) LCPs, the chains are clustered into nematic domains each having a different director. Coalescence of such domains proceeds by the gradual local rotation and alignment of directors between neighboring domains, commonly considered as the annihilation mechanism of disclinations in nematic melts (Figure 5.9, left) ${ }^{44}$. However, in the scenario where chain-extended LCPs are constrained (i.e. with a high molecular 
weight and a physical network established by reactive coupling of the prepolymer), segments of LCP chains (formerly the LCPP chains) are limited in their mobility in all directions by either neighboring chains or coupled chain segments that potentially bridge nematic domains (Figure 5.9, right). These constraints impose strong limitations on how both LCP chains and their respective chain segments move, and hinder the reorientation of chain segments that is required for the coalescence of domains. Whether the constrained nematic state of the LCPs with higher molecular weights results in a large delay or the complete prevention of the interchain relaxation is currently unclear. Further research is required to evaluate the extent to which different parameters, such as viscosity and state of the melt, play a role in delaying the relaxation of interchain orientation in LCPs.

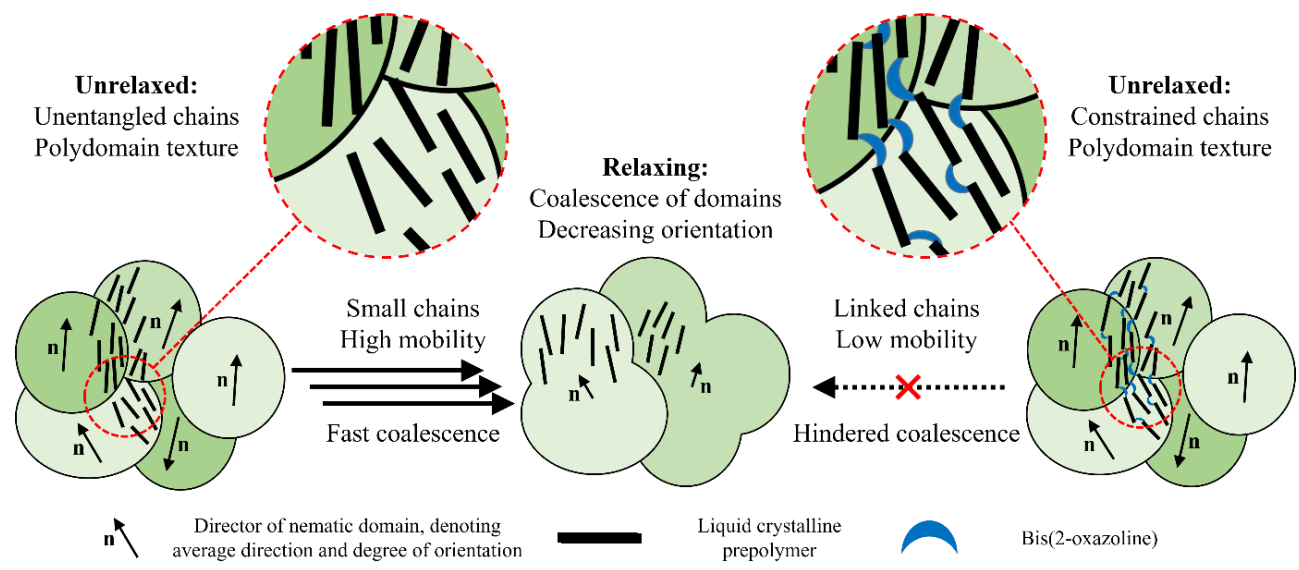

Figure 5.9 Schematic representation of the role chain extension in delaying or preventing coalescence of domains and the concomitant relaxation of the orientation parameter.

\section{Mechanical properties of injection molded bars}

The mechanical properties of the LCPP and the chain extended polymers are evaluated in tension on injection-molded samples. Table 5.2 shows the Young's modulus $(\mathrm{E})$, stress at break ( $\left.\sigma_{\text {break }}\right)$, strain at break $\left(\varepsilon_{\text {break }}\right)$, and orientation parameter $(\mathrm{S})$ of different LCPs. The E-modulus increases significantly upon chain extension (7.2 GPa for LCPP, compared to $12.5 \mathrm{GPa}$ for CE-2:1), but is found to be very similar for all chain-extended LCPs. Such comparable tensile moduli are expected as all chainextended LCPs have comparable chemical constituents and the same orientation parameter after processing. The lower E-modulus for LCPP is the result of a lower orientation parameter, originating its relatively low viscosity and correspondingly faster relaxation upon injection molding. In contrast, the stress at break increases significantly with increasing molecular weight, likely resulting from a decreasing number of defects 
in the form of chain ends. Furthermore, the gradual increase in stress- and strain at break observed for CE-3:2, CE-1:1, and CE-3f, indicates an improved ability to transfer stresses through the material, which might be attributed to the presence of a physical network on molecular level in these samples. Overall, chain extension is found to improve the mechanical performance of the LCPP greatly, as illustrated by the stressstrain curves (Figure 5.10). In short, the chosen approach, based reactive extrusion, proved effective to produce LCPs with controllable molecular weights and viscosity and excellent mechanical performance.

Table 5.2 Mechanical properties and orientation parameter of produced LCPs after injection moldig. Note, values were averaged over 5 specimens and the standard deviation is provided.

\begin{tabular}{|c|c|c|c|c|}
\hline Material & $\mathrm{E}(\mathrm{GPa})$ & $\sigma_{\text {break }}(\mathrm{MPa})$ & $\varepsilon_{\text {break }}(\%)$ & $\mathrm{S}(-)$ \\
\hline LCPP & $7.2 \pm 0.14$ & $85 \pm 14$ & $1.6 \pm 0.33$ & 0.59 \\
\hline CE-2:1 & $12.5 \pm 0.24$ & $164 \pm 19$ & $2.2 \pm 0.75$ & 0.72 \\
\hline CE-3:2 & $12.8 \pm 0.25$ & $199 \pm 16$ & $3.6 \pm 0.99$ & 0.72 \\
\hline CE-1:1 & $12.8 \pm 0.17$ & $216 \pm 15$ & $4.2 \pm 0.96$ & 0.71 \\
\hline CE-3f & $12.7 \pm 0.17$ & $219 \pm 8.8$ & $4.2 \pm 0.11$ & 0.71 \\
\hline
\end{tabular}

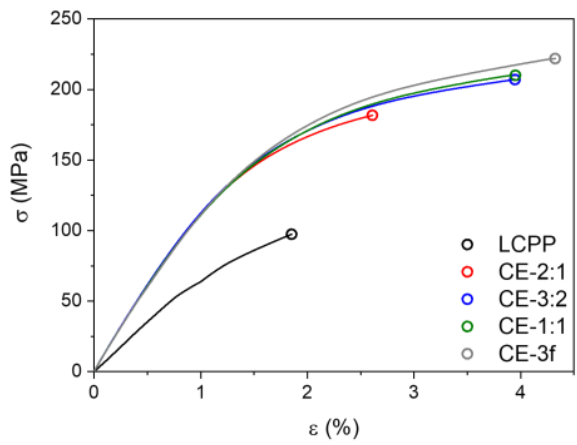

Figure 5.10 Characteristic stress-strain curves of injection-molded bars, at room temperature, comparing LCPP and the chain-extended LCPs obtained by reactive extrusion.

The mechanical performance of the LCPs produced in this study is compared to that of commercially available thermotropic polyesters. The performance of LCPs depends on the microstructure and degree of interchain orientation. Considering that the LCPs performance is influenced by the processing conditions, similar processing conditions are required in the comparative study. In Chapter 3, injection molded bars of the commercially available Vectra LCP V400P grade were made using the injection molding set up used in this study, albeit at a higher processing temperature? ${ }^{9}$ The obtained 
injection molded bars had a Young's modulus of $17 \mathrm{GPa}$ and a stress at break of 250 $\mathrm{MPa}$. To ensure a fair comparison with the studies reported in literature, we consider studies that describe the mechanical properties of unfilled LCPs along the flow direction having a thickness in the range of 1 to $3 \mathrm{~mm}$. These studies indicate that injection molded parts produced with commercially available LCPs have a Young's modulus and stress at break ranging from 8.6 to $12 \mathrm{GPa}$ and from 160 to $230 \mathrm{MPa}$, respectively ${ }^{45-48}$. Therefore, we can conclude that the chain extended LCPs produced in this study have comparable mechanical performance to the commercially available thermotropic polyesters, with the added benefit of a versatile backbone structure that attributes to lower processing temperatures.

\subsection{Conclusions}

In this study we reported on a reactive extrusion route based on chain extension of a liquid crystalline prepolymer using bis(2-oxazoline) chemistry for the development of thermotropic liquid crystalline polyesters with controllable molecular weight. The mechanical performance of the produced LCPs is comparable to that of commercially available thermotropic polyesters, with a Young's modulus and tensile strength over 12.5 GPa and $200 \mathrm{MPa}$, for injection molded bars. The relatively low viscosity and polymerization temperature results in less stringent requirements for the LCP synthesis and allow for the incorporation of novel, bio-based, monomer into the LCP via a wellknown and viable route, being a conventional acidolysis polycondensation. With the developed method, we demonstrated control over molecular weight and chainarchitecture of the LCP, resulting in an increase in viscosity and relaxation time. Furthermore, as the molecular weight of the LCP increases, we observe a transition in the viscoelastic behavior from an unentangled nematic melt to a constrained melt. Interestingly, minimal relaxation of orientation parameter is observed upon heating the chain-extended LCPs to temperatures well above the glass transition temperature. This behavior deviates significantly from that of typical main-chain thermotropic polymers, which tend to undergo rapid relaxation of the nematic texture and interchain orientation following the cessation of flow. The delayed relaxation observed in the chain extended LCPs is attributed to the adopted synthesis route, which involves the coupling of chain ends under a complex flow field. This results in a unique conformation of the polymer chains, which influences their respective mobility and results in a constrained state and the formation of a physical network. 


\subsection{References}

(1) Chung, T.-S. The Recent Developments of Thermotropic Liquid Crystalline Polymers. Polym. Eng. Sci. 1986, 26 (13), 901-919. https://doi.org/10.1002/pen.760261302.

(2) Han, H.; Bhowmik, P. K. Wholly Aromatic Liquid-Crystalline Polyesters. Prog. Polym. Sci. 1997, 22 (7), 1431-1502. https://doi.org/10.1016/S0079-6700(96)00028-7.

(3) Acierno, D.; Mantia, F. P. La; Polizzotti, G.; Ciferri, A.; Valenti, B. Ultrahigh Modulus Liquid Crystalline Polyesters. Macromolecules 1982, 15 (6), 1455-1460. https://doi.org/10.1021/ma00234a001.

(4) Vita, F.; Adamo, F. C.; Pisani, M.; Heist, L. M.; Li, M.; Hegde, M.; Dingemans, T. J.; Samulski, E. T.; Francescangeli, O. Liquid Crystal Thermosets. A New Class of HighPerformance Materials. Liq. Cryst. 2019, 1-11. https://doi.org/10.1080/02678292.2019.1641233.

(5) Iqbal, M.; Dingemans, T. J. High Tg Nematic Thermosets: Synthesis, Characterization and Thermo-Mechanical Properties. Eur. Polym. J. 2010, 46 (11), 2174-2180. https://doi.org/10.1016/j.eurpolymj.2010.08.010.

(6) Iqbal, M.; Picken, S. J.; Dingemans, T. J. Synthesis and Properties of Aligned AllAromatic Liquid Crystal Networks. High Perform. Polym. 2014, 26 (4), 381-391. https://doi.org/10.1177/0954008313516986.

(7) Gantenbein, S.; Masania, K.; Woigk, W.; Sesseg, J. P. W.; Tervoort, T. A.; Studart, A. R. Three-Dimensional Printing of Hierarchical Liquid-Crystal-Polymer Structures. Nature 2018, 561 (7722), 226-230. https://doi.org/10.1038/s41586-018-0474-7.

(8) Montes de Oca, H.; Wilson, J. E.; Penrose, A.; Langton, D. M.; Dagger, A. C.; Anderson, M.; Farrar, D. F.; Lovell, C. S.; Ries, M. E.; Ward, I. M.; et al. Liquid-Crystalline Aromatic-Aliphatic Copolyester Bioresorbable Polymers. Biomaterials 2010, 31 (30), 7599-7605. https://doi.org/10.1016/j.biomaterials.2010.07.006.

(9) de Kort, G. W.; Rastogi, S.; Wilsens, C. H. R. M. Controlling Processing, Morphology, and Mechanical Performance in Blends of Polylactide and Thermotropic Polyesters. Macromolecules 2019, 52, 6005-6017. https://doi.org/10.1021/acs.macromol.9b01083.

(10) Padias, A. B.; Hall, H. K. Mechanism Studies of LCP Synthesis. Polymers (Basel). 2011, 3 (2), 833-845. https://doi.org/10.3390/polym3020833.

(11) Wilsens, C. H. R. M.; Verhoeven, J. M. G. A.; Noordover, B. A. J.; Hansen, M. R.; Auhl, D.; Rastogi, S. Thermotropic Polyesters from 2,5-Furandicarboxylic Acid and Vanillic Acid: Synthesis, Thermal Properties, Melt Behavior, and Mechanical Performance. Macromolecules 2014, 47 (10), 3306-3316. https://doi.org/10.1021/ma500433e.

(12) de Kort, G. W.; Bouvrie, L.; Rastogi, S.; Wilsens, C. H. R. M. Thermoplastic PLA-LCP Composites: A Route towards Sustainable, Reprocessable, and Recyclable Reinforced Materials. Sustain. Chem. Eng. 2019, 1-35. https://doi.org/10.1021/acssuschemeng.9b06305.

(13) Economy, J. Aromatic Polyesters of P-Hydroxybenoic Acid. Mol. Cryst. Liq. Cryst. 1989, 169, 1-22. https://doi.org/10.1080/00268948908062731.

(14) Demus, D.; Goodby, J.; Gray, G. W.; Spiess, H. W.; Vill, V. Handbook of Liquid Crystals; Wiley-VCH, 1998. https://doi.org/10.1002/9783527620593.

(15) Collyer, A. A. Thermotropic Liquid Crystal Polymers for Engineering Applications Applications. Mater. Sci. Technol. 1989, 5, 309-322. https://doi.org/10.1179/mst.1989.5.4.309.

(16) Culbertson, B. M. Cyclic Imino Ethers in Step-Growth Polymerizations. Prog. Polym. Sci. 2002, 27 (3), 579-626. https://doi.org/10.1016/S0079-6700(01)00053-3. 
(17) Luston, J.; Kronek, J.; Markus, O.; Janigova, I.; Böhme, F. Synthesis and Polymerization Reactions of Cyclic Imino Ethers. 3. Poly(Ester Amide)s of the AA+BB Type on the Basis of 2-Oxazolines. Polym. Adv. Technol. 2007, 18, 165-172. https://doi.org/10.1002/pat.801.

(18) Roy, M.; Noordzij, G. J.; Van Den Boomen, Y.; Rastogi, S.; Wilsens, C. H. R. M. Renewable (Bis)Pyrrolidone Based Monomers as Components for Thermally Curable and Enzymatically Depolymerizable 2-Oxazoline Thermoset Resins. ACS Sustain. Chem. Eng. 2018, 6 (4), 5053-5066. https://doi.org/10.1021/acssuschemeng.7b04716.

(19) Tzoganakis, C. Reactive Extrusion of Polymers: A Review. Adv. Polym. Technol. 1989, 9 (4), 321-330. https://doi.org/10.1002/adv.1989.060090406.

(20) Hong, S. M.; Hwang, S. S.; Seo, Y.; Chung, I. J.; Kim, K. U. Reactive Extrusion of In situ Composite Based on PET and LCP Blends. Polym. Eng. Sci. 1997, 37 (3), 646-652. https://doi.org/10.1002/pen.11708.

(21) Xanthos, M.; Dagli, S. S. Compatibilization of Polymer Blends by Reactive Processing. Polym. Eng. Sci. 1991, 31 (13), 929-935. https://doi.org/10.1002/pen.760311302.

(22) Xanthos, M.; Young, M. W.; Karayannidis, G. P.; Bikiaris, D. N. Reactive Modification of Polyethylene Terephthalate with Polyepoxides. Polym. Eng. Sci. 2001, 41 (4), 643-655. https://doi.org/10.1002/pen.10760.

(23) Jacobsen, S.; Fritz, H. G.; Degée, P.; Dubois, P.; Jérôme, R. New Developments on the Ring Opening Polymerisation of Polylactide. Ind. Crops Prod. 2000, 11 (2-3), 265-275. https://doi.org/10.1016/S0926-6690(99)00053-9.

(24) Machado, A.; Bounor-Legare, V.; Goncalves, N.; Melis, F.; Cassagnau, P.; Michel, A. Continuous Polymerization of e -Caprolactone Initiated by Titanium Phenoxide in a Twin-Screw Extruder. J. Appl. Polym. Sci. 2008, 110 (6), 3480-3487. https://doi.org/10.1002/app.

(25) Bras, W.; Dolbnya, I. P.; Detollenaere, D.; Van Tol, R.; Malfois, M.; Greaves, G. N.; Ryan, A. J.; Heeley, E. Recent Experiments on a Combined Small-Angle/Wide-Angle X-Ray Scattering Beam Line at the ESRF. J. Appl. Crystallogr. 2003, 36 (3 I), 791-794. https://doi.org/10.1107/S002188980300400X.

(26) Portale, G.; Cavallo, D.; Alfonso, G. C.; Hermida-Merino, D.; Van Drongelen, M.; Balzano, L.; Peters, G. W. M.; Goossens, J. G. P.; Bras, W. Polymer Crystallization Studies under Processing-Relevant Conditions at the SAXS/WAXS DUBBLE Beamline at the ESRF. J. Appl. Crystallogr. 2013, 46 (6), 1681-1689. https://doi.org/10.1107/S0021889813027076.

(27) Mitchell, G. R.; Windle, A. H. Orientation in Liquid Crystal Polymers. In Developments in crystalline polymers; Basset, D. C., Ed.; Elsevier Applied Science: London, UK, 1988. https://doi.org/10.1007/978-94-009-1341-7_3.

(28) Trejo-Machin, A.; Verge, P.; Puchot, L.; Quintana, R. Phloretic Acid as an Alternative to the Phenolation of Aliphatic Hydroxyls for the Elaboration of Polybenzoxazine. Green Chem. 2017, 19 (21), 5065-5073. https://doi.org/10.1039/c7gc02348k.

(29) Wilsens, C. H. R. M.; Wullems, N. J. M.; Gubbels, E.; Yao, Y.; Rastogi, S.; Noordover, B. A. J. Synthesis, Kinetics, and Characterization of Bio-Based Thermosets Obtained through Polymerization of a 2,5-Furandicarboxylic Acid-Based Bis(2-Oxazoline) with Sebacic Acid. Polym. Chem. 2015, 6 (14), 2707-2716. https://doi.org/10.1039/c4py01609b.

(30) Windle, A. H.; Viney, C.; Golombok, R.; Donald, A. M.; Mitchell, G. R. Molecular Correlation in Thermotropic Copolyesters. Faraday Discuss. Chem. Soc. 1985, 79, 55-72. https://doi.org/10.1039/DC9857900055. 
(31) Rudin, A.; Burgin, D. Effects of Molecular Weight and Chain Ends on Glass Transition of Polystyrene. Polymer (Guildf). 1975, 16 (4), 291-297. https://doi.org/10.1016/00323861(75)90173-1.

(32) Mackley, M. R. The Rheology and Micro-Structure of Flowing Thermotropic Liquid Crystal Polymers. Mol. Cryst. Liq. Cryst. Inc. Nonlinear Opt. 1987, 153 (1), 249-261. https://doi.org/10.1080/00268948708074541.

(33) Burghardt, W. R.; Brown, E. F.; Auad, M. L.; Kornfield, J. A. Molecular Orientation of a Commercial Thermotropic Liquid Crystalline Polymer in Simple Shear and Complex Flow. Rheol. Acta 2005, 44 (5), 446-456. https://doi.org/10.1007/s00397-004-0424-1.

(34) Beekmans, F.; Gotsis, A. D.; Norder, B. Transient and Steady-State Rheological Behavior of the Thermotropic Liquid Crystalline Polymer Vectra B950. J. Rheol. (N. Y. N. Y). 1996, 40 (5), 947. https://doi.org/10.1122/1.550788.

(35) Colby, R. H. Structure and Linear Viscoelasticity of Flexible Polymer Solutions: Comparison of Polyelectrolyte and Neutral Polymer Solutions. Rheol. Acta 2010, 49 (5), 425-442. https://doi.org/10.1007/s00397-009-0413-5.

(36) Acierno, D.; La Mantia, F. P.; Polizzotti, G.; Ciferri, A.; Krigbaum, W. R.; Kotek, R. Thermotropic Homopolyesters. Iv. Study of Fiber Formation. J. Polym. Sci. Part B Polym. Phys. 1983, 21 (10), 2027-2036. https://doi.org/10.1002/pol.1983.180211012.

(37) Wool, R. P. Polymer Entanglements. Macromolecules 1993, 26 (7), 1564-1569. https://doi.org/10.1021/ma00059a012.

(38) de Kort, G. W.; Leoné, N.; Stellamanns, E.; Auhl, D.; Wilsens, C. H. R. M.; Rastogi, S. Effect of Shear Rate on the Orientation and Relaxation of a Vanillic Acid Based Liquid Crystalline Polymer. Polymers (Basel). 2018, $10 \quad$ (9), 935. https://doi.org/10.3390/polym10090935.

(39) Beekmans, F.; Gotsis, A. D.; Norder, B. Influence of the Flow History on Stress Growth and Structure Changes in the Thermotropic Liquid Crystalline Polymer Vectra B950. Rheol. Acta 1997, 36, 82-95.

(40) Guo, T.; Harrison, G. M.; Ogale, A. A. Transient Shear Rheology and Rheo-Optical Microstructural Characterization of a Thermotropic Liquid Crystalline Polymer. Polym. Eng. Sci. 2005, 45 (2), 187-197. https://doi.org/10.1002/pen.20265.

(41) Langelaan, H. C. The Relaxation of Shear and Normal Stresses of Nematic Liquid Crystalline Polymers in Squeezing and Shear Flows. J. Rheol. (N. Y. N. Y). 1996, 40 (1), 107. https://doi.org/10.1122/1.550733.

(42) Romo-Uribe, A. Long-Range Orientation Correlations and Molecular Alignment in Sheared Thermotropic Copolyester. In Situ Light and X-Ray Scattering. Polym. Adv. Technol. 2007, 18, 503-512. https://doi.org/10.1002/pat.878.

(43) RomoUribe, A.; Lemmon, T. J.; Windle, A. H. Structure and Linear Viscoelastic Behavior of Main-Chain Thermotropic Liquid Crystalline Polymers. J. Rheol. (N. Y. N. Y). 1997, 41 (5), 1117-1145. https://doi.org/10.1122/1.550820.

(44) Hsiao, B. S.; Stein, R. S.; Deutscher, K.; Winter, H. H. Optical Anisotropy of a Thermotropic Liquid- crystalline Polymer in Transient Shear. J. Polym. Sci. Part B Polym. Phys. 1990, 28 (9), 1571-1588. https://doi.org/10.1002/polb.1990.090280912.

(45) Plummer, C. J. G.; Zülle, B.; Demarmels, A.; Kausch, H. - H. The Structure of Filled and Unfilled Thermotropic Liquid Crystalline Polymer Injection Moldings. J. Appl. Polym. Sci. 1993, 48 (5), 751-766. https://doi.org/10.1002/app.1993.070480501.

(46) Wang, Y. L.; Yue, C. Y.; Tam, K. C.; Hu, X. Relationship between Processing, Microstructure, and Mechanical Properties of Injection Molded Thermotropic LCP. J. Appl. Polym. Sci. 2003, 88 (7), 1713-1718. https://doi.org/10.1002/app.11846. 
A reactive processing route to thermotropic polyesters with low processing temperature and enhanced relaxation time.

(47) Suokas, E.; Sarlin, S.; Tömälä, P. The Relationship Between the Microstructure and Physical Properties of Injection Molded Naphthalenic-Based Liquid Crystal Copolyester. Mol. Cryst. Liq. Cryst. Inc. Nonlinear Opt. 1987, 153, 515-524. https://doi.org/10.1080/00268948708074562.

(48) Heynderickx, I.; Paridaans, F. Influence of Processing Conditions on the Anisotropy in Injection-Moulded Thermotropic LCPs. Polymer (Guildf). 1993, 34 (19), 4068-4074. https://doi.org/10.1016/0032-3861(93)90668-Z. 


\subsection{Supporting Information}

\section{H-NMR Analysis}

The integrated ${ }^{1} \mathrm{H}-\mathrm{NMR}$ spectrum of $\mathbf{L C P P}$ is shown in Figure S5.1. The ${ }^{1} \mathrm{H}-\mathrm{NMR}$ spectra of the acetylated monomers and the bis(2-oxazoline) chain extender are shown in Figure S5.2 and S5.3. In Figure S5.1, three regions of the spectrum are integrated, and the obtained values are compared to the expected values based on the monomer composition in Table S5.1. The doublet around $3.18 \mathrm{ppm}$, and its shoulder at $2.86 \mathrm{ppm}$ correspond to the aliphatic $\mathrm{CH}_{2}$ groups of monomer $\mathbf{C}$. The singlet at $4.02 \mathrm{ppm}$ corresponds to the methoxy-group of monomer $\mathbf{B}$. The region corresponding to the aromatic protons of the used monomers (6.7 - 8.6 ppm) was integrated as a whole, due to the overlap of the signals from the different monomers and the presence of a peak corresponding to solvent (chloroform, $7.28 \mathrm{ppm}$ ). All the integrations were normalized with respect to the expected peak area of the $\mathrm{CH}_{2}$ groups of monomer $\mathbf{C}$. The obtained peak areas matched well with the calculated values from the used monomer composition, indicating that the produced LCPP had the intended composition.

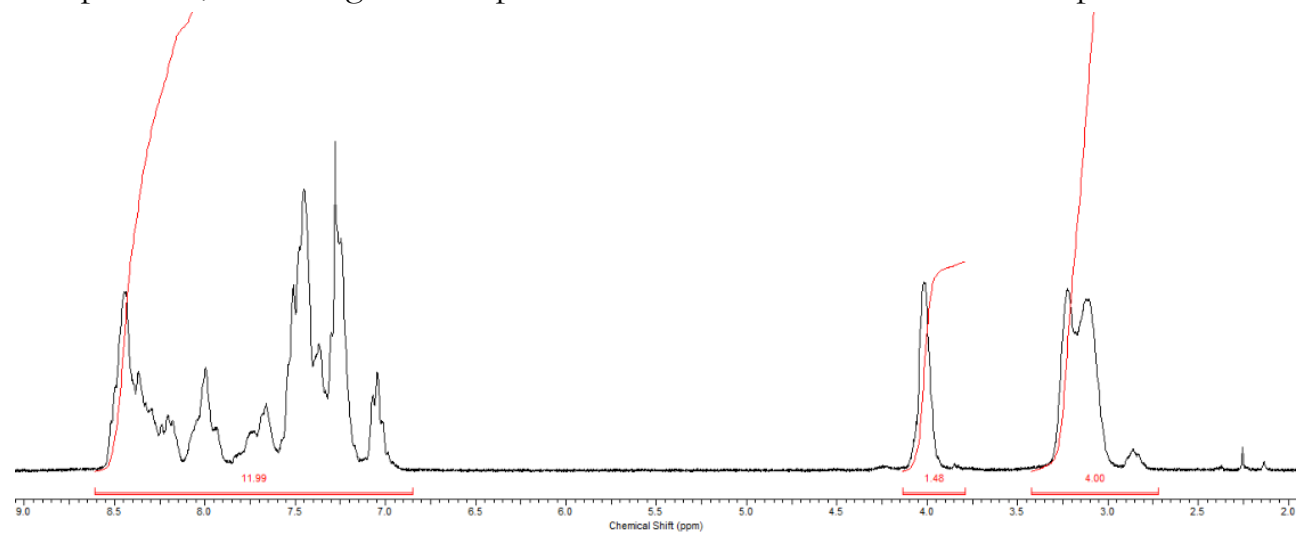

Figure S5.1 Integrated ${ }^{1} H-N M R$ spectrum of LCPP, measured in a mixture of $C D C l_{3} / d-T F A$. 
A reactive processing route to thermotropic polyesters with low processing temperature and enhanced relaxation time.

Table S5.1 Analysis of the monomer content of LCPP, based on ${ }^{1} H$-NMR. The obtained peak areas are based on the integration of the ${ }^{1} H$-NMR spectrum, as shown in Figure S5.1.

\begin{tabular}{|c|c|c|c|c|}
\hline Monomer & $\begin{array}{c}\text { Feed } \\
\text { content } \\
(\mathrm{mol} \%)\end{array}$ & $\begin{array}{c}\text { Expected peak area } \\
\text { (Aromatic } \\
\text { Aliphatic) }\end{array}$ & $\begin{array}{c}\text { Obtained peak } \\
\text { area } \\
\text { (Aromatic) }^{\mathrm{b}}\end{array}$ & $\begin{array}{c}\text { Obtained } \\
\text { peak area } \\
\text { (Aliphatic) }\end{array}$ \\
\hline $\begin{array}{c}\text { para-acetoxybenzoic } \\
\text { acid (A) }\end{array}$ & 32.10 & $4 / 0$ & - & 0 \\
\hline $\begin{array}{c}\text { O-acetylvanillic acid } \\
(\mathrm{B})\end{array}$ & 16.05 & $1.5 / 1.5$ & - & 4.48 \\
\hline $\begin{array}{c}\text { 3-(4-acetoxyphenyl) } \\
\text { propanoic acid (C) }\end{array}$ & 32.10 & $4 / 4$ & - & 0 \\
\hline $\begin{array}{c}\text { meta-acetoxybenzoic } \\
\text { acid (D) }\end{array}$ & 16.05 & $2 / 0$ & - & 0 \\
\hline terephthalic acid (E) & 3.70 & $0.35 / 0$ & 11.99 & 5.48 \\
\hline Total sum & 100 & $11.85 / 5.5$ & - & 0 \\
\hline
\end{tabular}

${ }^{a}$ The expected peak areas are normalized based on the composition in such a way that the 4 aliphatic protons of $(\boldsymbol{C})$ result in a signal of 4.

${ }^{b}$ Due to overlap between the numerous aromatic signals the peak areas of individual protons or monomers could not be determined. The total peak area of the aromatic region $(7.8-8.6 \mathrm{ppm})$, including a relatively small peak due to $\mathrm{CDCl}_{3}(7.28 \mathrm{ppm})$, is reported.

a

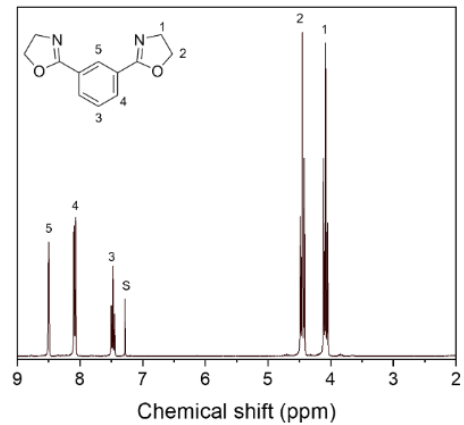

b

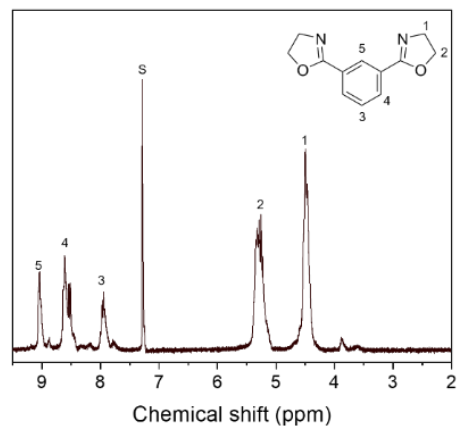

Figure S5.2 (a) ${ }^{1} \mathrm{H}$-NMR spectrum of isophthalic bis(2-oxazoline) (IaOx), measured in $\mathrm{CDCl}_{3}$. (b) ${ }^{1} \mathrm{H}$ NMR spectrum of isophthalic bis(2-oxazoline) (IaOx), measured in a mixture of $\mathrm{CDCl}_{3} / \mathrm{d}-\mathrm{TFA}$. 
a

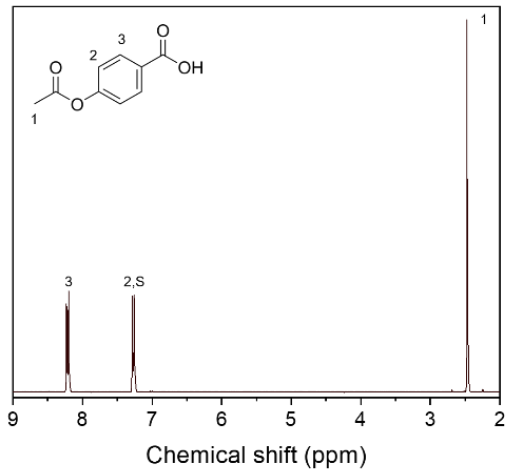

c

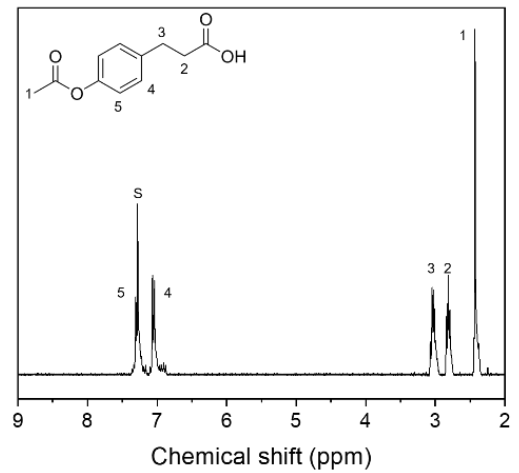

e

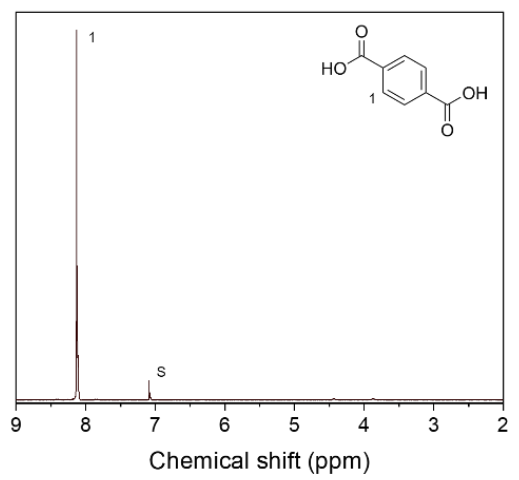

b

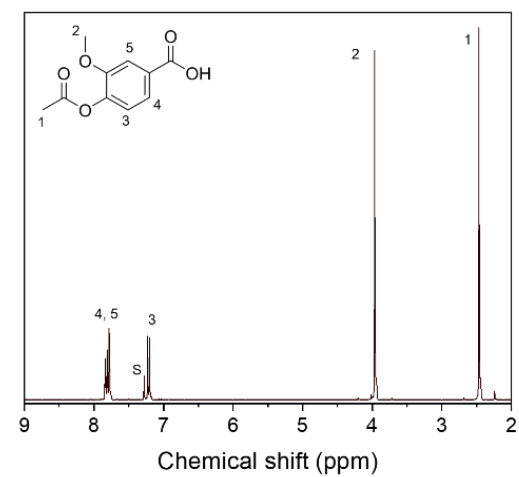

d

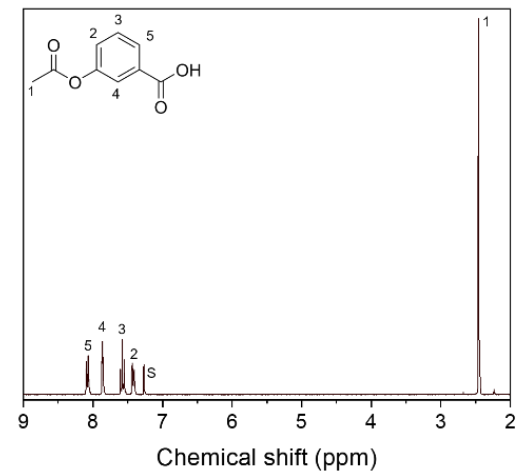

Figure S5.3 (a) ${ }^{1} H$-NMR spectra measured in a mixture of $C D C l_{3} / d$-TF $A$ of (a) para-acetoxybenzoic acid (monomer A), (b) o-acetylvanillic acid (monomer B), (c) 3-(4-acetoxyphenyl)propanoic acid (monomer C), (d) meta-acetoxybenzoic acid (monomer D), (e) terephthalic acid (monomer E). 


\section{Temperature dependence of the moduli of LCPP and CE-3f}

The dynamic moduli of LCPP and CE-3f were measured as the samples were cooled from $175{ }^{\circ} \mathrm{C}$ at a rate of $5{ }^{\circ} \mathrm{C} \min ^{-1}\left(\omega=10 \mathrm{rad} \mathrm{s}^{-1}\right.$, Figure S5.4a). A TA Instruments Discovery HR 2 rheometer with a parallel plate geometry was used. The glass transition temperatures and the observed shift upon chain extension (marked by the arrow) match well with those observed in DSC. Both LCPs show a gradual change in their respective moduli upon cooling, indicating no formation of crystallites and demonstrating the amorphous nature of these materials. These measurements also reflect the transition form an unentangled nematic melt to a constrained melt that occurs upon crosslinking. In the case of $\mathbf{L C P P}$, the viscous modulus (G') exceeds the elastic modulus ( $\left.G^{\prime}\right)$ at any given temperature above $T_{g}$, as is characteristic for unentangled melts. CE-3f on the other hand shows three different regimes typically observed in constrained polymer melts: a rouse regime $\left(G^{\prime \prime}>G^{\prime}\right.$ for $\left.\mathrm{T}=99-117^{\circ} \mathrm{C}\right)$, a plateau regime $\left(G^{\prime}>G^{\prime}\right.$ for $\mathrm{T}$ $\left.=117^{\circ} \mathrm{C}-167^{\circ} \mathrm{C}\right)$, and a terminal regime $\left(\mathrm{G}^{\prime \prime}>\mathrm{G}^{\prime}\right.$ for $\left.\mathrm{T}>167^{\circ} \mathrm{C}\right)$.

(a)

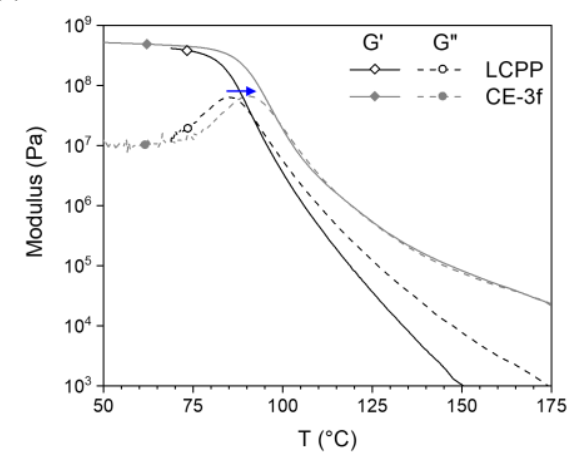

(b)

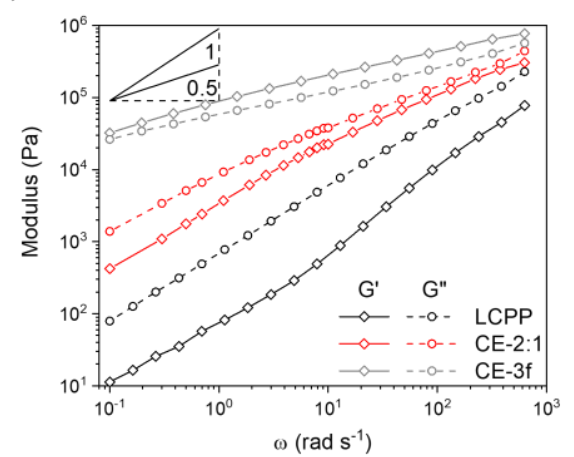

Figure S5.4 (a). Storage and loss modulus as a function of temperature of LCPP and CE-3f, measured at $\omega$ $=10 \mathrm{rad} \mathrm{s}^{-1}$. The blue arrow marks the difference in $T_{\mathrm{g}}$ between the two materials, as determined by the maxima in G”. (b) Storage and loss modulus as function of angular frequency of LCPP, CE-2:1, and CE-3f, measured at $150{ }^{\circ} \mathrm{C}$. The lines in the top left corner mark slopes of 1 and 0.5 respectively.

\section{Storage and loss modulus as function of angular frequency of LCPP, CE-2:1, and CE-3f}

The viscoelastic behavior of $\mathbf{C E - 3 f}$ is shown in Figure S5.4b. CE-3f has a branched structure, as a trifunctional carboxylic acid was present during chain extension. This results in a physical network as the elastic modulus exceeds the viscous modulus over the entire measured frequency range and a longer relaxation time compared to the other LCPs, as the crossover point, marking the transition to the terminal regime, is expected to occur below $\omega<10^{-1} \mathrm{rad} \mathrm{s}^{-1}$. LCPP and CE-2:1 are shown for comparison. 


\section{D-WAXD diffraction patterns}
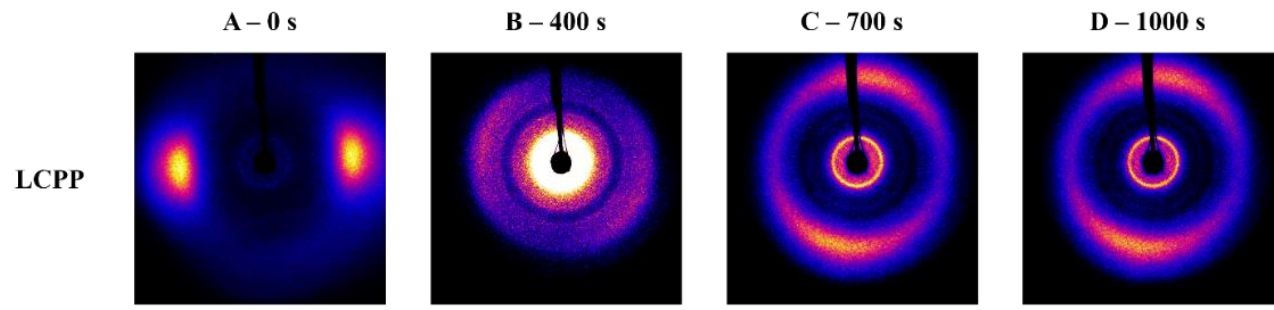

CE-2:1
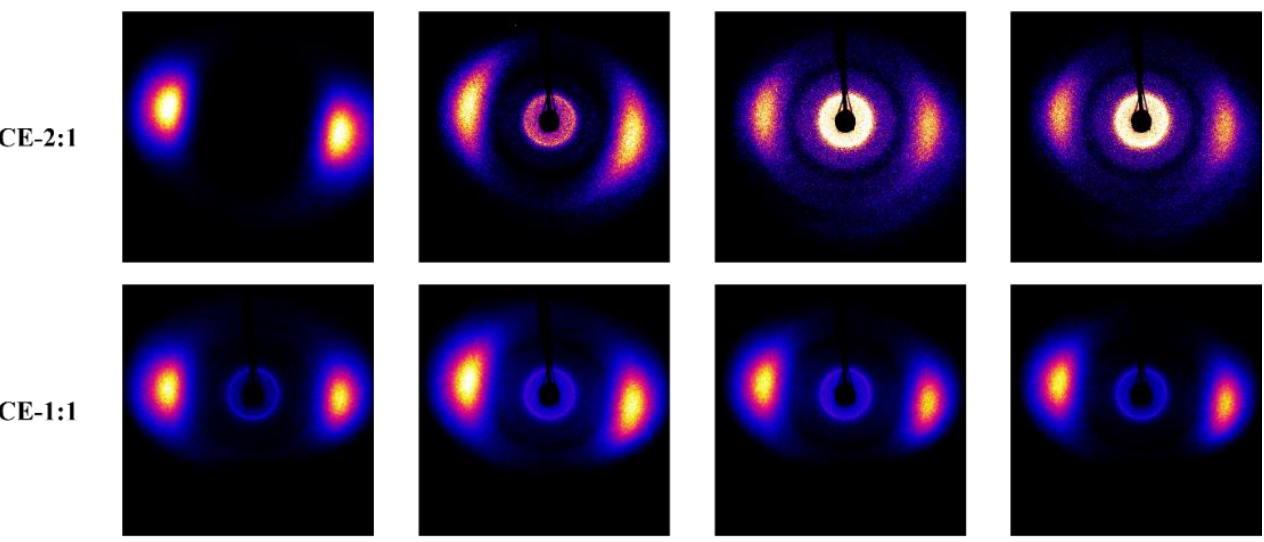

$\mathrm{T}\left({ }^{\circ} \mathrm{C}\right)$

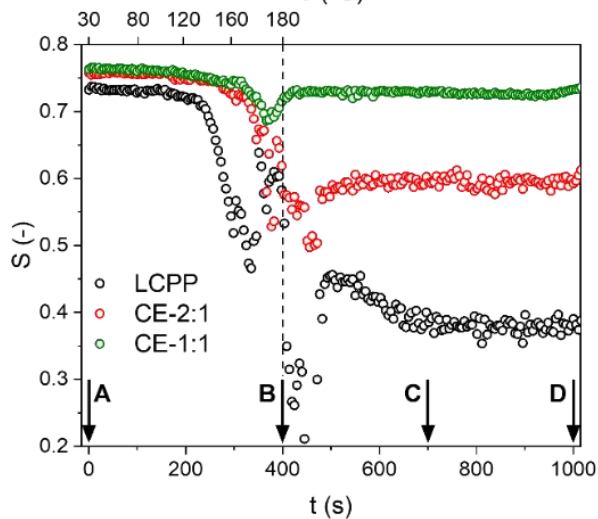

CSS 450 shear cell and polyimide film

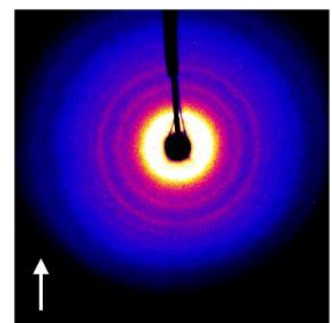

Arrow marks the drawing direction

Figure S5.6 (Top) Diffraction patterns melt-drawn tapes for the respective LCPs, taken at $0 s(\boldsymbol{A}), 400 s$ $(\boldsymbol{B}), 700 s(\boldsymbol{C})$, and $1000 s(\boldsymbol{D})$ after the start of heating. The drawing direction is vertical. (Bottom left) Relaxation of the orientation parameter (S) of prepared LCP tapes upon heating. The moments the shown diffraction patterns were taken are marked $\boldsymbol{A}-\boldsymbol{D}$. (Bottom right) Diffraction pattern of the modified shear cell including a number of layers of polyimide tape that was used. 
Diffraction patterns of LCPP and the respective chain extended LCPs, taken at specific times during the relaxation of the interchain orientation, are shown in Figure S5.6. To evaluate the relaxation of the interchain orientation of the LCPs, a stack of melt-drawn tapes was wrapped in polyimide-tape and heated to $180^{\circ} \mathrm{C}$ at a rate of 30 ${ }^{\circ} \mathrm{C} \mathrm{min}^{-1}$, followed by an isothermal period of 10 minutes. The samples were continuously irradiated by a synchrotron X-ray source in order to assess development of the microstructure. The drawing direction is vertical with respect to the diffraction patterns. Since the samples were not placed into the shear cell exactly vertical, a tilt of several degrees is typically observed. Diffraction patterns obtained at the start of heating (A, $0 \mathrm{~s})$, after reaching the isothermal temperature (B, $400 \mathrm{~s})$, after annealing for $5 \mathrm{~min}$ (C, 700 s), and after annealing for $10 \mathrm{~min}(\mathbf{D}, 1000 \mathrm{~s})$ are shown.

Initially, the diffraction patterns of all LCPs display a broad diffraction signal, characteristic for an amorphous phase, and the azimuthal arcs indicate a significant level of interchain orientation. As the width of the arcs, and therefore orientation is comparable, the relaxation of the LCPs started from a similarly oriented state. Over time, the tapes were heated above their $T_{g}$, resulting in a decreasing viscosity and increased mobility. During this period (between 300 and 500 s) the stacked tapes fuse and flow slightly, most likely resulting in inconsistencies in the data and the observed undershoot in $\mathrm{S}$ in this regime. This also led to a decrease in sample thickness and signal intensity over time. This seems to be more pronounced in the samples with lower viscosities. The effect is particularly clear in the case of LCPP, as due to flow, the original direction of the orientation, which was the result of melt drawing, is lost. Note that for the calculation of the orientation parameter $(S)$ the maximum azimuthal intensity was taken as the orientation direction, therefor the observed shift in orientation direction has not resulted in negative values of $\mathrm{S}$.

It was found that an increase in $M_{w}$ due to chain extension resulted in a later onset of relaxation and that larger degree of orientation was maintained upon annealing. LCPP relaxes quickly upon heating, loses its original direction of orientation and retains a final $S$ around 0.4. Chain extended CE-2:1 and CE-3:2 relax considerably slower, and retain the original direction of orientation as well as a higher degree of orientation (S 0.55 0.6). Hardly any changes are observed in the diffraction patterns of the LCPs with the highest molecular weights (CE-1:1, and CE-3f), indicating slow relaxation and retention of a high degree of orientation. This is likely related to the increased viscosity and constrained state of the nematic melt of these samples. 
Chapter 5 


\section{Chapter 6 - The importance of viscosity control for recyclable reinforced thermoplastic composites.}

\section{Abstract}

Thermoplastic composites consisting of a liquid crystalline polymer (LCP) and poly(lactide) (PLA) combine good mechanical performance with recyclability, and are therefore interesting as strong and sustainable composite materials. The viscoelastic behavior of both the LCP and the PLA is important, as they determine the LCP morphology and play a crucial role in preventing loss of mechanical performance upon recycling. The effect of the matrix viscosity is well documented in literature, systems where the LCP viscosity is tailored are not reported. Therefore, four LCPs with the same chemical backbone and different molecular weights are used to produce reinforced LCP-PLA composites. The differences in LCP viscosity and viscosity ratio between the dispersed phase and the matrix of the blends are evident in the composite morphology: The fibril diameter increases as the viscosity ratio increases. The diameter ranges from several hundred nanometer to a few micrometer. Typical layered structures are observed in injection molded, where the layer-thickness is influenced by the LCP viscosity. The LCPs are found to effectively reinforce the PLLA matrix, increasing the Young's modulus by $60 \%$ and the maximum stress by $40 \%$ for the composite containing $30 \mathrm{wt} \%$ of the most viscous LCP. Remarkably, this did not result in an increase in brittleness, effectively increasing the toughness of the composite compared to pure PLLA. The feasible reprocessability of this composite is demonstrated, by subjecting it to three reprocessing cycles. The relaxation of the LCPs orientation upon heating is measured via in situ WAXD. We compare the relaxation of the LCP interchain orientation in an amorphous PLA matrix and in a semi-crystalline PLLA matrix. The matrix viscosity is found to influence the relaxation. In an amorphous matrix, relaxation of the LCP fibrils into droplets dominates the process, whereas a semi-crystalline matrix maintains the fibril morphology. In the latter case, the LCPs relax via contraction and coalescence of the polydomain texture. LCP orientation persists until the PLLA crystals melt. The insights gained in this study on the role of the LCP viscosity on the morphology and performance of thermoplastic composites, and the relaxation of LCPs in a matrix, aids progression towards sustainable and reprocessable thermoplastic composites.

\section{Published as:}

de Kort, G. W.; Saidi, S.; Hermida-merino, D.; Leoné, N.; Srinivas, V.; Rastogi, S.; Wilsens, C. H. R. M. Importance of Viscosity Control for Recyclable Reinforced Thermoplastic Composites. Macromolecules, 2020, 53, 6690-6702. 


\subsection{Introduction}

Fiber-reinforced composites are a versatile class of materials, combining excellent mechanical properties with low density. These materials contribute to a more sustainable future, as their high specific properties allow, for example, weight saving and higher fuel efficiency in the transportation sector and novel structural components in construction ${ }^{1}$. However, at the end of their use, following failure of the composite, these materials are typically less sustainable as their recycling proves challenging ${ }^{2-4}$; The most common recycling strategies are mechanical recycling, and fiber recovery. In mechanical recycling, the composite is ground and reprocessed. This process breaks and damages the reinforcing fibers, resulting in a decreased performance as the performance of the composite depends strongly on length and diameter of the reinforcing phase $\mathrm{e}^{5-8}$. Fiber recovery involves removal of the matrix phase, eg. using a harmful solvent or via pyrolysis, although this process is generally resource intensive and the performance of the recovered fibers is not always comparable to the original fibers ${ }^{9,10}$.

Thermoplastic reinforced composites, such as composites based on liquid crystalline polymers (LCPs) and poly(lactide) (PLA), can provide a solution to this issue. These materials provide good mechanical performance, comparable to short glass fiberreinforced composites, while being compatible with thermomechanical recycling ${ }^{11-13}$. In thermoplastic composites, both the reinforcing phase and the matrix phase are meltprocessable, where the mechanical performance depends on the morphology of the dispersed phase (length, diameter, distribution) formed during melt processing, similar to the fiber morphology dependency in regular fiber-reinforced systems ${ }^{14,15}$. In Chapter 4, we have demonstrated that the morphology and mechanical performance of an LCPPLA composite remain constant irrespective of the number of reprocessing cycles, given that the viscosity of the dispersed LCP phase remains lower than that of the PLA matrix (i.e. viscosity ratio $<1$ ).

Thermotropic LCP's are melt processable and are known for their excellent mechanical properties and low viscosity ${ }^{16,17}$. Both these properties stem from the rigid nature of the polymer backbone, allowing the LCP chains to be oriented on a molecular level by flow. This makes these materials highly suitable to serve as the reinforcing phase in thermoplastic composites: as the LCP droplets are deformed by the thermoplastic matrix in a flow field, elongated fibrils are formed with the LCP chains molecularly oriented along the fibril direction. This results in the production of reinforced composites, given that this fibrillar LCP morphology can be maintained upon cooling. The morphology and chain orientation of the LCP are key parameters for the performance of LCP-reinforced composites ${ }^{13}$. Therefore, the mechanical performance 
is strongly governed by the processing conditions and, more importantly, the viscoelastic properties of both of the blends constituents.

The high processing temperatures $\left(>300^{\circ} \mathrm{C}\right)$ of commercial thermotropic LCPs and the lack of control over the viscosity of these materials limit their use in thermoplastic composite applications. The former hinders their use (and re-use) as reinforcing component in many thermoplastic matrices which generally suffer from a lower thermal stability, whereas the latter generally results in a high viscosity ratio for the blend $(\lambda>$ 1 , due to the high $T_{m}$ of the LCP), which hinders the formation of the required fibrillar morphology. In general, the influence of the viscosity ratio on the blend morphology has been well studied. However, for LCPs in a thermoplastic matrix the emphasis has been on changing the matrix ${ }^{18,19}$. Due to their complex viscoelastic behavior an approach based on altering the LCPs viscosity could provide valuable insight, however, these are scarce in literature as the molar mass is not easily controlled via typical acidolysis polycondensation. In Chapter 5 a route was developed to obtain thermotropic LCPs with good mechanical performance, a low processing temperature and a tunable molecular weight, based on a thermal ring-opening addition reaction between carboxylic acid terminated LCP polymers and bis(2-oxazolines). In this Chapter, we make use of these LCPs to generate LCP-PLA composites and to investigate the effect of the LCP viscosity on the composite morphology and performance. The relaxation of the blends upon heating and the effect of the matrix phase are investigated via in situ Wide-angle $\mathrm{X}$-ray Diffraction combined with polarized optical microscopy.

\subsection{Methods and Materials}

\section{Materials}

The semi-crystalline poly(L-lactide) (PLLA) grade was purchased from Total Corbion (Purapol L130) and the amorphous PLA grade was obtained from NatureWorks (Ingeo Biopolymer 6302D). The used LCPs were produced in house. The synthesis of the liquid crystalline prepolymer (LCPP) via acidolysis polycondensation and the production of the chain extended LCPs via reactive extrusion is reported in Chapter 5.

\section{Preparation and Processing of PLA-LCP Blends}

The PLA pellets, PLLA pellets and previously produced LCPs were dried overnight (in vacuo, $60{ }^{\circ} \mathrm{C}$ ) prior to use. Mixtures with containing $30 \mathrm{wt} \%$ LCP of the different LCPs and PLLA/PLA were prepared and fed in a preheated DSM Xplore twin-screw micro-extruder with a barrel size of $5 \mathrm{~mL}$ for extrusion. The recycle channel and allows control over the residence time, and a valve can be switched to expel the material from the barrel. The materials were mixed for 3 minutes at $190{ }^{\circ} \mathrm{C}$ and $100 \mathrm{rpm}$. After 
extrusion, the samples were either processed directly into melt-drawn filaments or transferred into a preheated barrel and injection molded into tensile bars. Melt-drawn filaments were prepared using a slit die mounted at the extruder outlet $(0.5 \times 3 \mathrm{~mm}$, produced by DSM Xplore) and a winder (Dienes SD-type). The tapes were drawn and cooled in air. A DSM Xplore IM 5.5 micro injection molder was used to produce tensile bars $(2 \mathrm{~mm} \times 4 \mathrm{~mm} \times 70 \mathrm{~mm}$, with a gage length of $25 \mathrm{~mm}$ ). The barrel temperature was set to $190^{\circ} \mathrm{C}$, while the mold temperature was set to $25^{\circ} \mathrm{C}$. An injection pressure of 8 bar was used for all samples. The same processing conditions were used to reprocess the CE-1:1-PLLA composite thrice. The tensile bars were cut into pieces in between the processing cycles.

\section{Material Characterization}

The molecular weight distributions of the liquid crystalline prepolymer and the chain extended LCPs were determined via gel permeation chromatography (GPC) using a PSS SECcurity GPC system with Agilent 1260 Infinity instrument technology. The system is equipped with two PFG combination medium micro-columns with $7 \mu \mathrm{m}$ particle size (4.6 x $250 \mathrm{~mm}$, separation range 100-1.000.000 Da), a PFG combination medium precolumn with $7 \mu \mathrm{m}$ particle size $(4.6 \times 30 \mathrm{~mm})$, and a Refractive Index detector (RI). Distilled 1,1,1,3,3,3-hexafluoroisopropanol (HFIP) containing $0.019 \%$ sodium trifluoroacetate was used as mobile phase at $40{ }^{\circ} \mathrm{C}$, with a $0.3 \mathrm{~mL} \mathrm{~min}{ }^{-1} \mathrm{flow}$ rate. The obtained molecular weight distributions are relative with respect to poly(methyl methacrylate) standards obtained from PSS. Samples were prepared via dissolution of approximately $6 \mathrm{mg}$ of LCP in $1.5 \mathrm{ml}$ HFIP. The samples were shaken overnight and subsequently filtered over a $0.2 \mu \mathrm{m}$ PTFE syringe filter prior to injection.

The viscoelastic behavior of the PLLA, PLA, and the LCPs was determined in a MCR 702 TwinDrive rheometer (Anton Paar) with a parallel plate geometry (diameter of 12 $\mathrm{mm}$, gap of $0.7 \mathrm{~mm}$ ). The PLLA samples were loaded at $190{ }^{\circ} \mathrm{C}$ and kept isothermal for 3 minutes to erase mechanical and thermal history. Subsequently the samples were either heated or cooled at a rate of $5{ }^{\circ} \mathrm{C} \min ^{-1}$ to the measurement temperature and subjected to a frequency sweep with a strain of $0.5 \%$. The LCPs were subjected to the same procedure with the exception that the samples were kept isothermal at $190{ }^{\circ} \mathrm{C}$ for 10 minutes.

The microstructure of the LCP-P(L)LA composites was evaluated via polarized optical microscopy, 2D-SAXS analysis and SEM analysis. The injection molded bars were cut into thin slices (thickness of 1.0 and $2.5 \mu \mathrm{m}$, respectively) along the injection direction with a Leica EM UC7 ultramicrotome. These samples were studied via polarized optical microscopy (POM) using an Olympus BX53 Microscope (5 or 50 
times magnification) equipped with an Olympus DP26 camera and a $530 \mathrm{~nm}$ retardation plate. To study the changes in morphology upon heating, slices with a thickness of 1.0 $\mu \mathrm{m}$ were cut along the drawing direction and Linkam HFSX350 temperature controlled stage was mounted on the microscope. The slices were placed between two glass plates and heated at a rate of $30{ }^{\circ} \mathrm{C} \mathrm{min}^{-1}$ to $180{ }^{\circ} \mathrm{C}$ while their optical morphology was monitored. Two-dimensional (2D) Small-angle X-ray Scattering (SAXS) analysis was performed on the LCP-PLLA injection-molded bars. A SAXSLAB Ganesha diffractometer was used with a sample-to-detector distance of $1076.3 \mathrm{~mm}$, using $\mathrm{Cu} \mathrm{K}$ $\alpha$ radiation $(\lambda=1.5406 \AA)$ and silver behenate $\left(\mathrm{d}_{001}=58.380 \AA\right)$ as calibrant. Scanning electron microscopy (SEM) imaging was done on the fracture surfaces of the LCPPLLA composites using a Philips X30 microscope at an acceleration voltage of $15 \mathrm{kV}$ and a magnification of $15000 \mathrm{x}$. The fracture surfaces were created by breaking samples cooled in liquid nitrogen, which were subsequently mounted and plasma-coated with a thin layer of gold prior to analysis.

The mechanical properties of the injection molded LCPs were assessed under tension. Measurement were performed on a Zwick Z100. Samples were subjected to a constant deformation rate of $5 \mathrm{~mm} \mathrm{~min}^{-1}$, at room temperature.

Wide-angle X-ray Diffraction (WAXD) measurements (wave length, $\lambda=0.104 \mathrm{~nm}$ ) were carried out at the European Synchrotron Radiation Facility (ESRF), the DUBBLE beamline (BM26B, Grenoble, France). The DUBBLE beamline is optimized for polymer science as is reported by Bras et al. ${ }^{20}$ and Portale et al. ${ }^{21}$. WAXD patterns, with an exposure time of 5 s, were collected using a Frelon detector $(2048 \times 2048$ pixels of $48.8 \mu \mathrm{m} \times 48.8 \mu \mathrm{m})$ placed at a distance of $0.18 \mathrm{~m}$. The data were normalized for synchrotron beam fluctuations using an ionization chamber placed before the sample. Furthermore, a correction for the sample absorption was performed using a photodiode located at the beamstop before the background contribution was subtracted. The wavenumber $\mathrm{q}=4 \pi \sin \theta / \lambda$, with $\theta$ being half of the scattering angle for WAXD experiments scale calibration, has been achieved by $\alpha-\mathrm{Al}_{2} \mathrm{O}_{3}$ (alumina). The samples were loaded into a Linkam CSS 450 shear cell, of which the glass windows were substituted by polyimide film to ensure low scattering of the device. The shear cell was used to study the relaxation of the interchain orientation of the LCPs. A stack of melt drawn tapes was wrapped in polyimide-tape (kapton $®)$. The stack of melt-drawn tapes was heated to $180{ }^{\circ} \mathrm{C}$ at a rate of $30{ }^{\circ} \mathrm{C} \mathrm{min}-1$, followed by an isothermal period of 10 minutes. The orientation parameter $(\mathrm{S}),\left\langle\mathrm{P}_{2 \mathrm{n}}(\cos \varphi)\right\rangle_{\mathrm{d}}$, was calculated from the obtained diffraction patterns via a procedure by Mitchell and Windle ${ }^{22}$, as described in Chapter 2 . 


\subsection{Results and Discussion}

In Chapter 5, we have demonstrated a method to obtain thermotropic liquid crystalline polymers with a low processing temperature and tunable molecular weight. To recall, a Liquid Crystalline PrePolymer (LCPP), with a low molecular weight, is synthesized via a conventional acidolysis polycondensation. Subsequently, a chain extension reaction with the LCPP and a bis(2-oxazoline) derived from isophthalic acid $(\mathbf{I a O x})$ is performed in a reactive extrusion process. During the chain extension reaction the 2-oxazoline moieties of the $\mathbf{I a O x}$ react with the carboxylic acid end groups of the LCPP, thus linking different LCPP chains, producing LCPs with increased molar mass. The resulting molar mass can controlled via the ratio LCPP : IaOx. All synthesized LCPs can be considered nematic glasses, since no crystallization is observed upon cooling or annealing. The LCPs exhibit a nematic phase above their respective $T_{\mathrm{g}}$ and the nematic ordering persists upon cooling below Tg. The nematic to isotropic transition could not be observed due to thermal degradation prior to the transition.

The synthesized prepolymer (LCPP) and the resulting chain extended LCPs have excellent mechanical properties, in combination with low processing temperatures and tunable viscosity. Therefore, these materials are suitable candidates as the reinforcing phase of sustainable and reprocessable thermoplastic reinforced composites as will be demonstrated in this chapter. Composites consisting of a PLLA matrix and four different LCPs, being the liquid crystalline prepolymer (LCPP), and three different chain extended LCPs (CE-2:1, CE-3:2, and CE-1:1, names are based on the ratio LCPP : IaOx), are prepared and analyzed. The molar mass data of the different LCPs is provided in Table 6.1.

Table 6.1 Molar ratio, molecular weight $\left(M_{w}\right.$ ) and polydispersity (PDI) (as measured by GPC) for the LCPs used in this study.

\begin{tabular}{|c|c|c|c|}
\hline Material & Molar ratio (LCPP $: \mathrm{IaOx})$ & $M_{\mathrm{w}}\left(\mathrm{kg} \mathrm{mol}{ }^{-1}\right)$ & $P D I(-)$ \\
\hline LCPP & $1: 0$ & 6.3 & 6.0 \\
\hline CE-2:1 & $2: 1$ & 15.3 & 9.7 \\
\hline CE-3:2 & $3: 2$ & 20.2 & 11.7 \\
\hline CE-1:1 & $1: 1$ & 40.5 & 15.6 \\
\hline
\end{tabular}

\section{Processing and development of blend morphology}

The performance of LCP reinforced thermoplastic composites is highly dependent on the LCP morphology which is in turn determined by the viscosity of the respective constituents of the blend, and the processing conditions ${ }^{13,23-25}$. A fibrillar LCP morphology ensures sufficient surface area to transfer stresses from the matrix and load the reinforcing LCP phase, and is a prerequisite for effective reinforcement ${ }^{11,14}$. During 
the formation of the fibrils the initially spherical LCP droplets are stretched in a flow field and the LCP chains are oriented. The LCP-PLA composites are prepared via extrusion, where the LCP phase is dispersed in the PLLA matrix through droplet breakup resulting from the complex shear and extensional flow fields. Next, the sample is injection molded where shear flow combined with cooling can create and preserve the desired fibrillar LCP morphology. For the LCP droplets to break up and to be finely dispersed in the PLA matrix, the droplets need to deform. The capillary number $(x$, Eq. $1)$, the ratio between the hydrodynamic forces and the interfacial forces acting on the droplet, must exceed unity for the droplets to stretch. Upon stretching, the diameter of the LCP droplet decreases until a critical value of the capillary number is reached ( $\left.x_{\text {crititcal }}\right)$ and the increased surface area of the stretched droplet is no longer stabilized by the hydrodynamic forces: the stretched fibril breaks up into smaller drops. Due to the increasing number of droplets formed during this breakup process, the number of droplet collisions increases. Colliding drops can then coalesce to form a larger droplet. Given sufficient time, the rate of coalescence and breakup will become equal, and an equilibrium morphology is established.

$$
\begin{gathered}
\kappa=\frac{\eta_{\text {matrix }} \cdot \dot{\gamma} \cdot d}{v_{12}} \\
\lambda=\frac{\eta_{L C P}}{\eta_{\text {matrix }}}
\end{gathered}
$$

Deformation and breakup of droplets in a matrix are affected by several additional factors. The critical capillary number, as depicted in Figure 6.1 is dependent on the viscosity ratio ( $\lambda$, Eq. 2 ) and the type of flow (eg. shear, or extension). For a droplet to stretch effectively in shear flow field, $\lambda$ close to unity or lower is required. Higher values of $\lambda$ gradually lead to less effective deformation of droplets, until at $\lambda \sim 3.8$ the timescale for the deformation increases to the extent that the rotational component of the shear flow field rotates the drops instead. Deformation of droplets can be hindered by elastic contributions in the flow behavior of the dispersed phase, due to the increased energy input required for deformation. In contrast, an extensional flow field is effective in deforming droplets regardless of the viscosity ratio of the blend. 


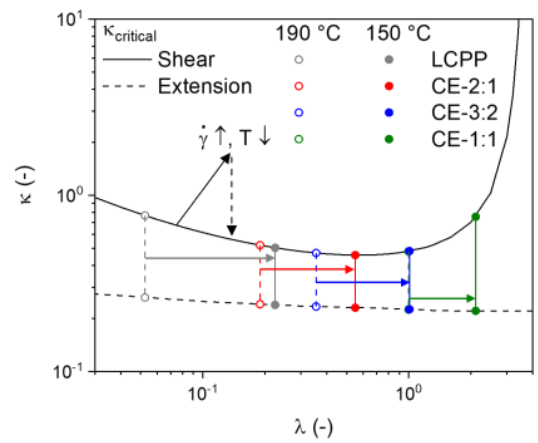

Figure 6.1 Evolution of the viscosity ratio of different LCP-PLLA blends with temperature. The effect of this transition on the critical capillary number (xcritical), both in shear and extension, is denoted by the colored points and arrows. The black arrows denote the effect of the change in conditions that occurs upon injection. The viscosity ratio is taken at an oscillation frequency of $137 \mathrm{rad} \mathrm{s}^{-1}$.

Upon injection molding, the molten blend is subjected to a sudden increase in shear rate combined with fast cooling. As a result of these conditions, the capillary number of the LCP drops increases rapidly, and the viscosity ratio of the blend increases as well. Since the droplets can only deform into long fibrils when $x>>x_{\text {critical }}$ and $\lambda$ is sufficiently low, the resultant morphology strongly depends on the viscoelastic behavior of both the PLLA matrix and the different LCPs. For example, a low molecular weight LCP is more easily dispersed and stretched compared to an LCP with a higher molecular weight, due to the lower $\lambda$ of the system. To form a fibrillar morphology with an oriented LCP phase, the viscoelastic behavior and thermal dependency of both phases must be complementary with each other and the chosen processing conditions. A more elaborate overview on the development of blend morphology is provided in Chapter 3.

The molecular weight control obtained by chain extension of LCPP with IaOx, and the corresponding control over the viscoelastic behavior (Figure 6.2a) can effectively be used to generate an LCP phase with the desired viscosity required for processing with the selected PLLA matrix. In more detail, LCPP, having the lowest molar mass, hardly shows any shear thinning within the measured frequency range and a Newtonian plateau is observed over a broad frequency range. At low frequencies there is an increase in viscosity due to the nematic texture. For the chain extended LCPs, the shear thinning behavior and the increase in viscosity due to the nematic texture become increasingly more dominant as the molecular weight increases from CE-2:1 to CE-1:1. Correspondingly, the Newtonian plateau ceases to exist for the higher molecular weight LCPs. This transition with increasing molecular weight is commonly observed in LCPs $^{26,27}$. The viscoelastic behavior of the PLLA shows a Newtonian plateau combined 
with shear thinning behavior at high frequencies. The viscosity of the PLLA exceeds that of all LCPs over the complete measured frequency range, with the exception of CE-1:1. In the lower frequency range the viscosity of CE-1:1 is higher due to the nematic texture; however, in the higher frequency range, which is more relevant for processing, the viscosities of CE-1:1 and the PLLA are comparable. This indicates that, under extrusion conditions at $190{ }^{\circ} \mathrm{C}$, all of the LCPs have a value of $\lambda$ close to, or below unity (Figure 6.2b), and can be effectively dispersed in the PLLA matrix selected in this study. Note that all measurements are carried out in the linear viscoelastic regime (Figure S6.1). This does not correlate directly to the viscoelastic behavior under actual processing conditions that involve unknown flow fields and large deformation, but it does allow a good estimation of the droplet behavior under such conditions.

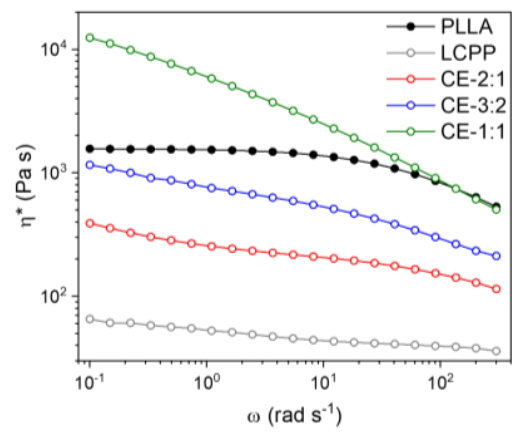

b

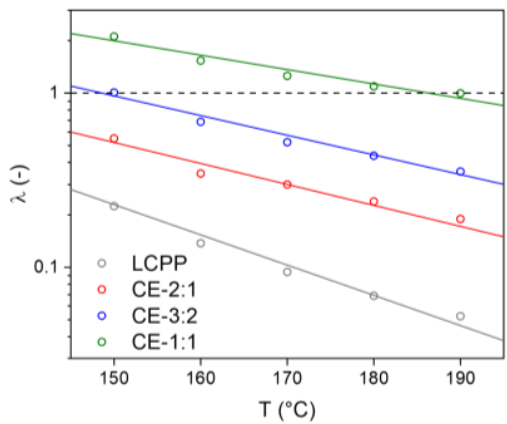

Figure 6.2 (a) Complex viscosity as a function of oscillation frequency for the PLLA and the different LCPs, measured at $190^{\circ} \mathrm{C}$. (b) Viscosity ratio of the blend consisting of PLL $A$ and the different LCPs as a function of temperature, determined at an oscillation frequency of $137 \mathrm{rad} \mathrm{s}^{-1}$.

As the temperature of the molten polymer decreases the viscosity changes accordingly, influencing the deformation of the LCP droplets. This is specifically relevant for injection molding, as the molten blend is not only deformed but also cooled. The viscosity ratio gradually increases upon cooling for all LCP-PLLA blends (Figure 6.2b), likely due to the fact that $T_{\mathrm{g}}$ of the LCPs is higher. Over the measured temperature range $\left(190{ }^{\circ} \mathrm{C}-150{ }^{\circ} \mathrm{C}\right)$ the viscosity ratio for the blends with LCPP and CE-2:1 does not exceed unity. For the blend with CE-3:2, $\lambda$ is close to unity over the whole temperature range and only exceeds unity at $150{ }^{\circ} \mathrm{C}$ and lower. The viscosity ratio for the blend of CE-1:1 and PLLA increases from unity to two as the temperature decreases to $150{ }^{\circ} \mathrm{C}$. Although deformation of the LCP droplets by the matrix becomes more challenging in this regime and droplets are stretched less efficiently, it can still occur under shear flow, as the limit $(\lambda \sim 3.8)$ is not reached. This implies that the LCP droplets can deform into fibrils in all different blends, even during cooling to $150^{\circ} \mathrm{C}$. 
The effect of changes in temperature and shear rate, as encountered in injection molding, on the deformability of the LCP droplets are visualized in Figure 6.1. Upon injection molding the shear rate increases drastically while the temperature decreases, resulting in an increase in both the viscosity ratio and the capillary number (as depicted by the black arrow). Given that the original viscosity ratio is not too high $(e g . \lambda<2)$, these changes result in a state where $x>>x_{\text {critical. }}$. This allows the LCP droplets to stretch into fibrils as long as the capillary number of the droplet remains larger than the critical value (as depicted by the dotted arrow). The fibrillar morphology is preserved upon further cooling, as both the LCP and the PLLA are quickly cooled below their respective glass transition temperatures.

The morphology of the injection molded bars, containing $30 \mathrm{wt} \%$ of the respective LCPs, is shown in Figure 6.3a. All morphological analysis is performed on the center of the sample, in the core region, as the morphology of the injection molded samples is known to change based on the distance from the mold wall. In all the blends, the LCP is present in the form of finely dispersed fibrils, as was expected from the viscoelastic behavior of the LCPs and PLLA. The uniform coloration of the fibrils viewed by polarized optical microscopy (Figure 6.3a) indicates a significant degree of interchain orientation of the LCP chains within the fibrils. Clear distinctions can be made with respect to the size and shape of the fibrils of the different LCPs. The LCPP fibrils are very thin, with diameters in the order of $600 \mathrm{~nm}$, and appear undulated. Due to its low molecular weight, LCPP has the lowest viscosity and proves to be the easiest to disperse in the PLLA matrix resulting in a very fine LCP fibril distribution. However, the low viscosity also results in shorter timescales for breakup of the droplets, likely causing the undulated appearance. Though the fibrils in the CE-2:1-PLLA blend are comparable in diameter, they are considerably longer and lack the undulated appearance of the LCPP fibrils. Due to the higher viscosity ratio compared to the LCPP-PLLA system, the CE2:1 fibrils do not break up as quickly as the LCPP fibrils, especially when the molten blend is cooled. The fibril morphology in the blend with CE-3:2 is, although still very fine (fibril diameters in the order of $1-2 \mu \mathrm{m}$ ), considerably more course. In part, this can be explained by the higher viscosity and viscosity ratio. Another contributing factor to the courser morphology is the presence of a physical network in the nematic melt of the chain extended LCPs with the highest molecular weights (CE-3:2 and CE-1:1); It is known that viscoelastic behavior of the dispersed phase hinders the deformation and breakup of droplets, compared to a purely Newtonian dispersed phase ${ }^{28,29}$. The physical network in the CE-1:1 and the relatively high viscosity ratio (upon cooling the deformation of the droplets is hindered) of this blend, lead to thicker and less stretched fibrils. It should be stated that even for the CE-1:1 blend the fibril diameter is around 
2-3 $\mu \mathrm{m}$, which is still considered a very fine dispersion. The observed length of the fibrils appears to be lower than the true length. This discrepancy is attributed to the sample preparation, as the fibrils are likely to be at an angle with respect to the cutting plane. This is supported by the microscopy images obtained from thicker slices (Figure S6.2, slice thickness of $2.5 \mu \mathrm{m}$ ), in which the fibrils are considerably longer. The difference in the morphology of the LCP-PLLA blends highlights the potential of a tunable molecular weight for the use of LCPs in thermoplastic reinforced composites: the viscosity of the LCP can be changed to obtain the optimal fibrillar morphology for a wide range of matrices.

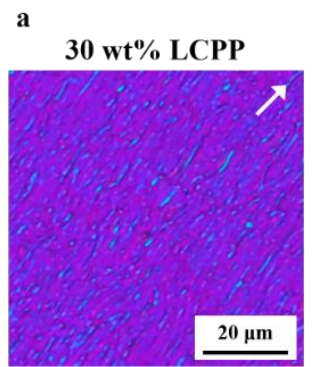

b

$$
30 \text { wt \% CE-1:1 }
$$

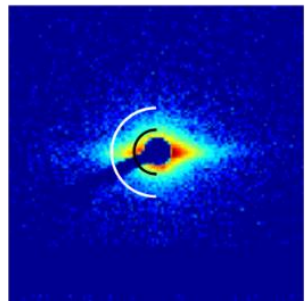

30 wt $\%$ CE-2:1

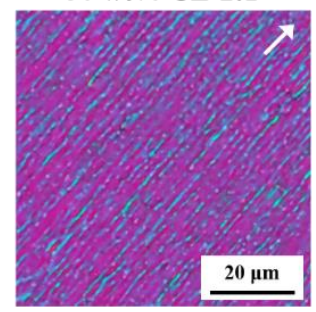

$30 w t \%$ CE-2:1
30 wt $\%$ CE-3:2

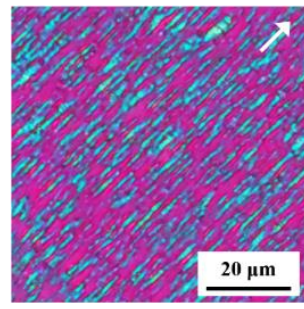

c
$30 \mathrm{wt} \%$ CE-1:1

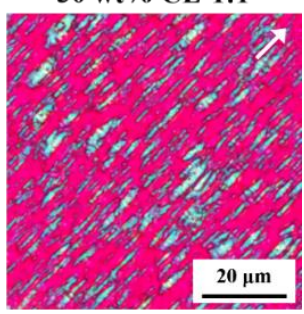

$30 w t \%$ CE-3:2

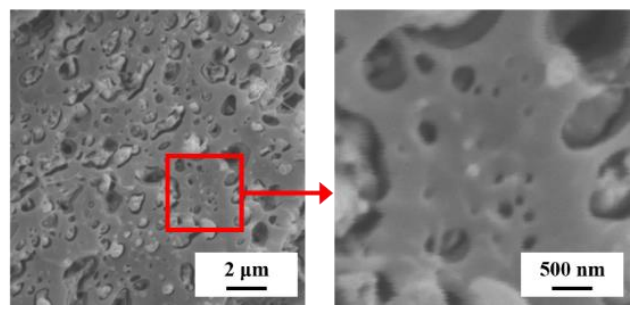

Figure 6.3 (a) Morphology of injection molded composites containing the different LCPs. The composites were microtomed to slices with a thickness of $1 \mu \mathrm{m}$. The images represent the core-region of the sample. The white arrows mark the injection molding direction. (b) SAXS patterns of the injection molded bars containing $30 w t \% C E$ 2:1 and CE-1:1. The white semicircle markes $q=0.125 \mathrm{~nm}^{-1}(d=50 \mathrm{~nm})$, whereas the black semicircle markes $q=0.0625 \mathrm{~nm}^{-1}(d=100 \mathrm{~nm})$. The white arrows mark the injection molding direction. (c) SEM images showing the LCP particle morphology of an injection molded composite containing $30 w t \%$ CE-3:2. The left image highlights the distribution of LCP particles with diameters in the micrometer range, whereas the enlarged image on the right highlights a second distribution of particles with sizes in the order of $100 \mathrm{~nm}$.

In addition to the LCP fibrils (viewed by optical microscopy), considerably smaller LCP particles (not visible in optical microscopy) appear to be present in all composites. Their presence is confirmed by Small-angle X-ray Scattering (Figure 6.3b) and scanning electron microscopy (Figure 6.3c). These particles have a diameter of $100 \mathrm{~nm}$ or smaller, and are likely to be related to the droplet breakup process. When LCP droplets are 
stretched to the extent the hydrodynamic forces do no longer balance out increasing surface forces the fibril becomes unstable and breaks up into very small droplets. Considering that the diameter of these droplets is very small, they can't be deformed by the flow field and can only disappear via coalescence. The small particles are very likely a combination of highly stretched fibrils that are close to instability, and the spherical droplets that are the result of breakup of the aforementioned fibrils. In SEM these particles appear nodular, indicating spherical droplets. The signal in SAXS shows a clear streak corresponding to structures oriented along the flow direction. The signal is stronger in composites with thinner fibrils $(30 \mathrm{wt} \%$ CE-2:1 compared to $30 \mathrm{wt} \% \mathbf{C E}$ 1:1), which indeed indicates the presence of oriented structures periodically spaced along the nanometer range $(\sim 100 \mathrm{~nm})$, suggesting that the signal comes from very thin fibrils aligned along the flow direction. Due to the origin of these very fine particles, it is likely that both very small spherical droplets and very thin stretched fibrils are present in the composites.

Injection molded parts typically have a morphology consisting of different layers due the fact that the cooling rate and shear rate vary with the distance to the molds wall ${ }^{30,31}$. Close to the wall, the melt experiences very high shear stresses and cooling rates, resulting in a shear layer. Towards the center of the sample, the stresses are lower and the cooling is slower, resulting in a core region with a different morphology. This local variation in conditions causes changes deformation of the LCP droplets by the PLA matrix, which results in the observation of several layers with different LCP morphologies ${ }^{13,32,33}$. Five distinct layers are observed in all different LCP-PLLA blends, as shown in Figure S6.3: 1) a quench layer in direct contact with the wall, 2) a shear layer close to the wall, 3) a transition layer a bit further from the wall, 4) a second transition layer, 5) a core region at the center of the sample. In the shear layer, where the material experiences very high stresses and cooling rates, the LCP is present in form of highly oriented ribbons. The first transition layer characteristically has shorter, irregular fibrils, as the stresses are reduced at this position but the cooling rates remain high. The second transition layer and core layer both have a fibrillar morphology; however, the fibrils in the second transition layer are notably thicker and longer. In both these layers, the cooling rate is relatively low, due to thermal insulation by the other layers, but the higher stresses in the transition layer allows the formation of longer fibrils. Due to the significant LCP loading in the blends (30 wt $\%$ ), the viscosity of the LCP has an effect on the overall viscosity. The viscosity of the melt is known to affect the thickness of the different layers in injection molding ${ }^{30}$. As the viscosity of the dispersed phase increases with the molecular weight of the LCP, so does the effective viscosity $\left(\eta_{\text {eff }}\right)$ of the blend. An overview of the thickness of the four layer for each of the LCP-PLLA composites 
is shown in Figure S6.4. The blend containing LCPP has the thickest shear layer, but relatively thin transition layers. As the viscosity of the LCP increases, the transition layers gradually grow thicker and the shear layer thins. The core decreases gradually in thickness as the LCP viscosity increases up to CE-1:1.

\section{Mechanical performance of developed blends}

The mechanical properties of the injection molded LCP-PLLA blends are compared to establish whether reinforcement by the LCP phase occurs, as would be expected from the fibrillar morphology. Indeed, the Young's (E) modulus increases upon the addition of any of the LCPs, while the maximum stress increases for the blends except for the blend containing LCPP (Figure 6.4 and Table 6.2). The LCP phase has a reinforcing effect in each of the blends, however it does so more effectively as its molecular weight increases; The most effective reinforcement is achieved in the blends containing CE-3:2 and CE-1:1, resulting in an increase of over 60\% in E-modulus and an increase of $40 \%$ in maximum stress. The LCPs with lower molecular weights (LCPP and CE-2:1) are relatively effective in increasing the modulus of the composite, but only the higher molecular weight LCPs (CE-3:2 and CE-1:1) effectively increase the maximum stress. In fiber-reinforced composites, it is often seen that such a trend is related to the morphology, more specifically the fiber length: short fibers improve the E-modulus, but longer fibers are required to increase the stress at break and impact resistance. In the case of these LCP-PLLA composites, however, it is unlikely that the morphology is the only factor, as the LCP is present as finely dispersed, highly stretched fibrils in each of the LCP-PLLA composites. Differences in the interchain orientation parameter $\left(\mathrm{S}_{\mathrm{LCP}}\right.$, Table 6.2) and the intrinsic mechanical properties of the LCPs have a dominant role on the mechanical response of each composites. As an example, LCPP, as a pure LCP, is considerably weaker than the chain extended LCPs at comparable levels of interchain orientation. The gradual increase in maximum stress of the composites appears to correlate strongly with the stress at break observed for the pure LCP materials. There is a slight decrease in E-modulus in the blend containing CE-1:1 compared to CE-2:1 and CE-3:2. However, is expected to be the result of the morphology: due to the relatively high viscosity ratio of the blend, deformation of the LCP droplets is somewhat hindered, resulting in a lower degree of orientation and concomitantly a lower E-modulus of the reinforcing phase. 
a

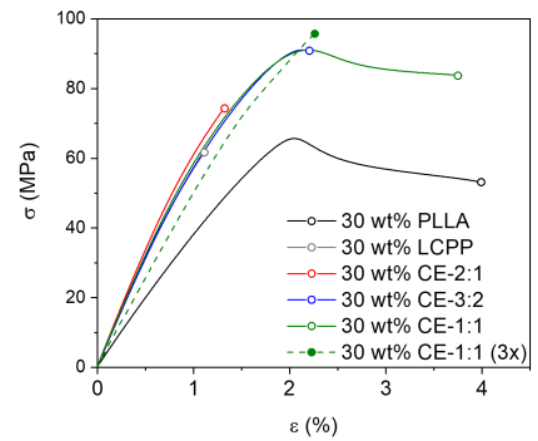

b

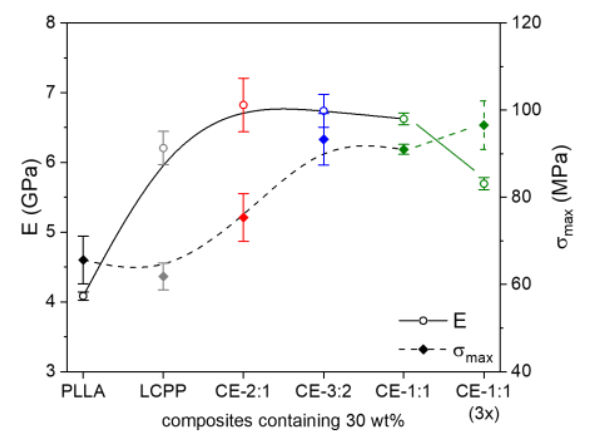

Figure 6.4 (a) Characteristic stress-strain curves for injection molded bars of pure PLLA, the different LCPPLLA composites, and the thrice reprocessed CE-1:1-PLLA composite. (b) Young's modulus and stress at break for injection molded bars of pure PLLA, the different LCP-PLLA composites, and the thrice-reprocessed CE-1:1-PLLA composite.

An improved tensile modulus and tensile strength accomplished by LCP reinforcement is typically accompanied by increased brittleness and thus lower strain at break. This is indeed the case for the composites containing LCPP and CE-2:1. However, the composites containing CE-3:2 and CE-1:1 prove relatively ductile (Figure 6.4a, Table 6.2), at least compared to the pure PLLA reference. The CE-3:2 composite reaches a yield point and subsequently break immediately. The CE-1:1 composites do actually yield and have a strain at break comparable to the pure PLLA: the composite is not only reinforced but also toughened. A very similar trend is found for the pure LCPs, where the higher molecular weight LCPs have a strain at break over $4 \%$. Not only are these relatively ductile reinforced composites interesting from an application perspective, but their ductility also provides information on LCP-PLLA interface. The fact that the stress and strain at break of the LCP-PLLA composites show the same trend as the pure LCPs indicates that strength of the LCP-PLLA interface is high, as the interfacial strength is not expected to vary significantly between the different LCPs. This is supported by SEM (Figure 6.3c), as the LCP fibrils are indeed broken at the fracture surface instead of pulled out. This also implies that the interfacial tension in the molten LCP-PLLA blends is low, which is in agreement with the very low diameter of the LCP fibrils after processing. 
Table 6.2 The mechanical properties and orientation parameter of the PLLA and the LCP-PLLA compositess after injection molding. The thrice reprocessed CE-1:1-PLLA composite is included. The mechanical performance of the pure LCPs is reported for comparison.

\begin{tabular}{|c|c|c|c|c|}
\hline Material & $\mathrm{E}(\mathrm{GPa})$ & $\sigma_{\max }(\mathrm{MPa})$ & $\varepsilon_{\text {break }}(\%)$ & $\mathrm{S}_{\mathrm{LCP}}(-)$ \\
\hline PLLA & $4.1 \pm 0.06$ & $65.6 \pm 5.5$ & $3.3 \pm 0.6$ & - \\
\hline $30 \mathrm{wt} \% \mathrm{LCPP}$ & $6.2 \pm 0.24$ & $61.8 \pm 3.1$ & $1.2 \pm 0.1$ & 0.67 \\
\hline $30 \mathrm{wt} \% \mathrm{CE}-2: 1$ & $6.8 \pm 0.39$ & $75.4 \pm 5.5$ & $1.4 \pm 0.1$ & 0.72 \\
\hline $30 \mathrm{wt} \% \mathrm{CE}-3: 2$ & $6.7 \pm 0.24$ & $93.3 \pm 5.9$ & $2.1 \pm 0.4$ & 0.72 \\
\hline $30 \mathrm{wt} \% \mathrm{CE}-1: 1$ & $6.6 \pm 0.09$ & $91.0 \pm 1.1$ & $3.1 \pm 0.6$ & 0.64 \\
\hline $30 \mathrm{wt} \%$ CE-1:1 & $5.7 \pm 0.09$ & $97.0 \pm 5.4$ & $2.2 \pm 0.2$ & 0.57 \\
\hline LCPP & $7.2 \pm 0.14$ & $85 \pm 14$ & $1.6 \pm 0.33$ & 0.59 \\
\hline CE-2:1 & $12.5 \pm 0.24$ & $164 \pm 19$ & $2.2 \pm 0.75$ & 0.72 \\
\hline CE-3:2 & $12.8 \pm 0.25$ & $199 \pm 16$ & $3.6 \pm 0.99$ & 0.72 \\
\hline CE-1:1 & $12.8 \pm 0.17$ & $216 \pm 15$ & $4.2 \pm 0.96$ & 0.71 \\
\hline
\end{tabular}

In order to mimic recycling and assess the reprocessability of these LCP-PLLA composites, the $30 \mathrm{wt} \% \mathbf{C E}-1: 1$ composite was reprocessed three times (sample $\mathbf{C E}$ 1:1 (3x)). Effective reinforcement is maintained, however, the Young's modulus and strain at break decrease upon reprocessing. This is likely caused by a coarser morphology, with less deformed fibrils and a lower LCP orientation. Due to thermal degradation of the PLLA, the viscosity ratio of the blend gradually increases, resulting in less effective deformation of the LCP in the molten blend ${ }^{25}$. Nevertheless, this is a promising result as significant reinforcement was maintained over three reprocessing cycles. To maintain the mechanical performance upon reprocessing, the use of a higher viscosity PLLA matrix (in combination with CE-1:1) or a different LCP (CE-3:2 with the same matrix) will be beneficial, as this prevents infeasible viscosity ratios upon reprocessing.

\section{Liquid crystalline polymer relaxation and its dependency on the matrix viscosity}

The degree of interchain orientation of the LCP $\left(\mathrm{S}_{\mathrm{LCP}}\right)$ is one of the key parameters influencing the mechanical performance of the LCP reinforced composites. The glassy nematic nature of the LCPP and the chain extended LCPs implies that the LCP will readily relax at temperatures above $T_{g}$, resulting in a rapid decrease in interchain 
orientation. However, in Chapter 5, we have found that the presence of a physical molecular network structure in chain extended LCPs limits relaxation as the LCPs retain a significant degree of interchain orientation, even after 10 minutes at temperatures over a $100{ }^{\circ} \mathrm{C}$ above $T_{g}$. This is a highly interesting property of these chain extended LCPs, however, it might not persist in composites as the surface tension provides an additional driving force for relaxation.

Generally, the textural relaxation of nematic LCPs occurs via the annihilation of disclinations: the coalescence of the polydomain texture results in the macroscopic decrease of interchain orientation ${ }^{34}$. Upon deformation, the nematic domains stretch and break up, and relaxation occurs via initial contraction followed by the slower coalescence process. Due to the smaller domains created at higher shear rates, the coalescence is accelerated ${ }^{35}$. In blends, the interfacial tension between the dispersed LCP phase and the PLA matrix phase can play a role and introduce different processes, such as fibril breakup, that influence the molecular relaxation of the LCP. The effect of the matrix on the relaxation of the interchain orientation of LCPP and the chain extended LCPs is investigated via Wide-angle X-ray Diffraction (WAXD). Melt drawn tapes, containing $30 \mathrm{wt} \%$ of the respective LCPs, are prepared on a lab-scale spinning set-up. Two different poly(lactide) grades were used as the matrix phase: a semicrystalline grade with a low D-isomer content (PLLA), and an amorphous grade with a higher D-isomer content (PLA). The molar mass distribution and viscosity of both grades are very similar (Figure S6.5). The melt-drawn tapes are wrapped in a polyimidetape and heated to $180{ }^{\circ} \mathrm{C}$ while collecting data at a synchrotron radiation source. The orientation parameter $\left(\mathrm{S}_{\mathrm{LCP}}\right)$ of the dispersed LCP is monitored and shown separately for tapes with the amorphous PLA matrix (Figure 6.5a) and the semi-crystalline PLLA matrix (Figure 6.5b). The orientation parameter at room temperature was similar for all samples $\left(\mathrm{S}_{\mathrm{LCP}} \sim 0.71\right)$, providing a good basis for comparison. In the amorphous matrix the interchain orientation starts to decrease slowly as the LCPs are heated above their respective $T_{g}$ 's, and the relaxation process accelerates as the samples are continuously heated. The observed relaxation rate depends on the viscosity of the LCP, as the chainextended LCPs with higher molecular weight relax slower. The behavior of the tapes with the PLLA matrix is quite different. Due to the slow crystallization kinetics and high cooling rates applied in the drawing process, the PLLA was initially amorphous. Upon heating the PLLA crystallizes (T $90-120^{\circ} \mathrm{C}$, t $100-150 \mathrm{~s}$ ). This is accompanied by a slight, temporary increase in the orientation parameter of all the LCPs, likely due to stresses imposed on the LCP fibrils by the shrinking PLLA matrix. Subsequently, the interchain orientation of the LCPs remains constant upon further heating, until the semi-crystalline PLLA melts and interchain orientation drops. 

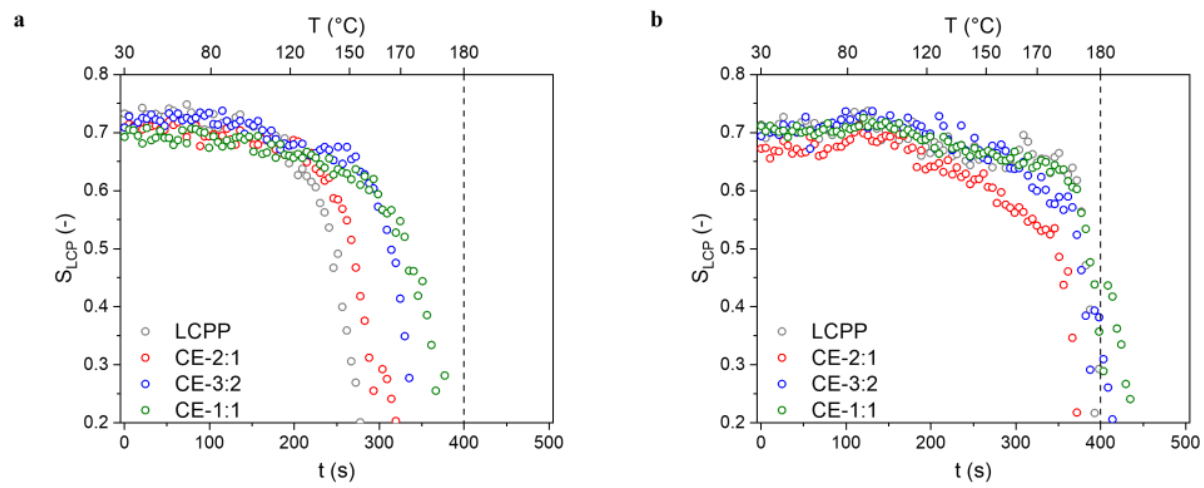

Figure 6.5 (a) Evolution of the orientation parameter ( $\left.S_{L C P}\right)$ of the different LCPs dispersed in (amorphous) $P L A$ tapes upon heating. (b) Evolution of the orientation parameter $\left(S_{L C P}\right)$ of the different LCPs dispersed in (semi-crystalline) PLLA tapes upon heating.

The relaxation of the interchain orientation of LCPs in a matrix differs significantly from relaxation the pure LCP, and is highly dependent on the matrix viscosity. When the matrix viscosity remains low upon heating (eg. amorphous PLA above its $T_{\mathrm{g}}$, Figure 6.5a) the decrease in interchain orientation appears not to be governed by the contraction and subsequent coalescence of the polydomain texture, as is observed in the pure LCPs. Instead, it appears that the relaxation of the fibrillar morphology is dominant, as the relaxation starts when both the LCP and the matrix are above their $T_{g}$. Additionally, there appears to be a strong dependence on the LCP viscosity as the LCP relaxation is delayed for blends having LCP with a higher molecular weight (Figure 6.5a). However, in the case the matrix crystallizes upon heating (eg. PLLA, Figure 6.5b), relaxation of the fibrillar morphology is not possible, and the LCPs retain a significant degree of orientation until the matrix melts. This is supported by optical microscopy (Figure 6.6), as microtomed slices of tapes consisting of CE-1:1 in both the PLA and PLLA matrices are heated, while the changes in morphology are captured. In the amorphous PLA matrix, the fibrils can gradually relax as the temperature increases. In the semi-crystalline PLLA matrix, oriented PLLA crystals are formed upon heating, as indicated by the increased birefringence at $125^{\circ} \mathrm{C}$. The crystallized matrix is so rigid that it does not allow relaxation of the fibrils. Only as the crystals are completely molten and do no longer stabilize the elongated shape of the fibrils, relaxation to spherical droplets and the concomitant relaxation of the interchain orientation can occur. Until this point, the birefringence of the CE-1:1 fibrils remains unchanged, indicating that the interchain orientation is maintained upon heating, as is the case for the pure LCPs. 

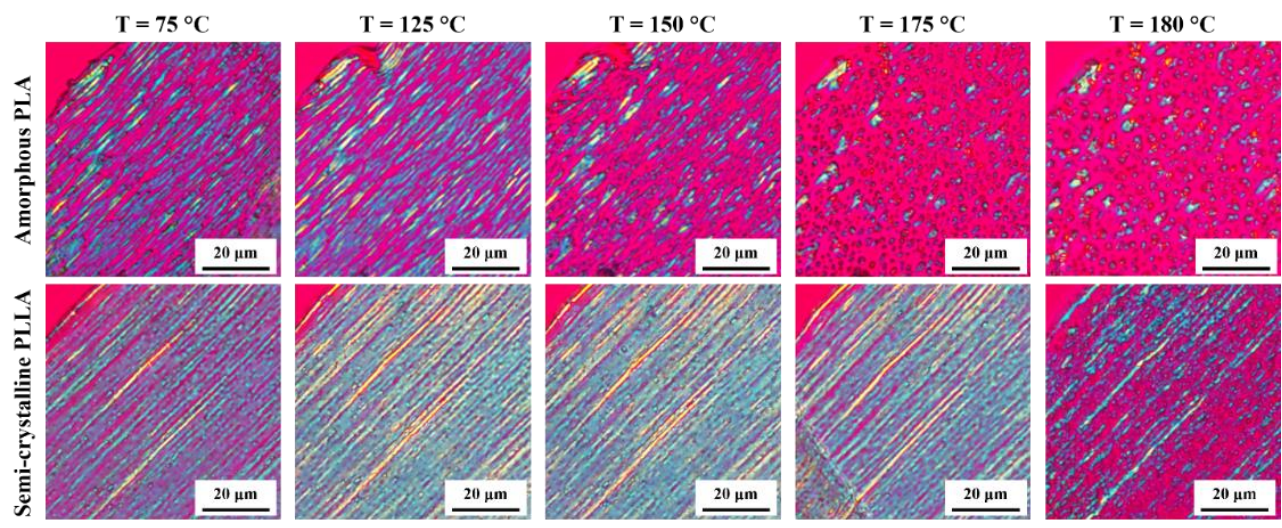

Figure 6.6 Evolution of the fibrillar morphology of the LCP dispersed in different matrices upon heating: an amorphous PLA matrix (top) and a semi-crystalline PLLA matrix (bottom).

In the absence of a matrix phase, a significant degree of interchain orientation is maintained upon heating especially as the molecular weight of the LCP increases. In the presence of a matrix, however, additional parameters (eg. interfacial tension, matrix viscosity, and LCP viscosity) play a significant role. As previously described, relaxation of the interchain orientation of the LCP embedded in a matrix strongly resembles that of the pure LCPs, given that the matrix is either solid or viscous enough to prevent or drastically delay the relaxation of the fibrillar morphology. Therefore, the LCP reinforced tapes with the semi-crystalline PLLA matrix are heated to $175^{\circ} \mathrm{C}$, below the full melting temperature of the PLLA crystals, and annealed for 10 minutes (Figure 6.7).

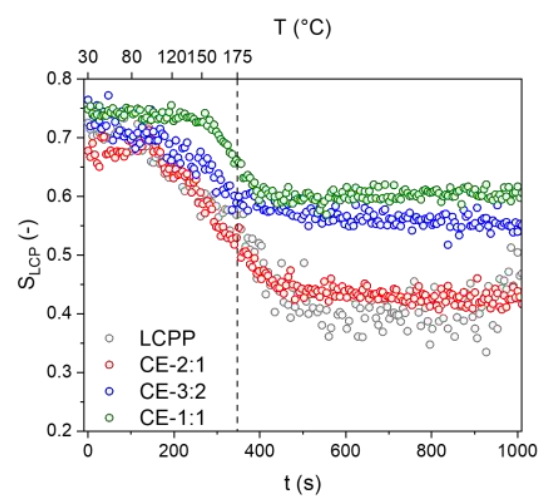

Figure 6.7. Evolution of the orientation of the different LCPs dispersed in a semi-crystalline PLLA matrix upon beating the tapes to $175^{\circ} \mathrm{C}$.

Under these conditions, some interchain orientation of the LCPs is maintained, even after annealing. This behavior is similar to that of the pure LCPs and the same trends with respect to the molecular weight of the LCPs are observed: in a low molecular 
weight LCP (eg. LCPP) the onset of relaxation occurs at a relatively low temperature and the decrease in $\mathrm{S}_{\mathrm{LCP}}$ is larger, whereas the onset for relaxation for higher molecular weight LCPs (eg. CE-1:1) is at a higher temperature and a larger degree of orientation is retained.

The evolution of the LCP interchain orientation in different tapes and at different annealing temperatures is shown for CE-2:1 (Figure 6.8a) and CE-1:1 (Figure 6.8b) in order to compare the relaxation behavior. Compared to the pure LCP the relaxation of the interchain orientation occurs at lower temperatures in the blends, regardless of whether the matrix phase crystallizes upon heating or not. The extent of the relaxation is also larger in the case the LCP is dispersed in a matrix. The earlier onset of relaxation in LCP-PLLA blends can potentially be explained via the matrix viscosity. In the case of the pure LCPs, relaxation of a nematic domain requires mobility in neighboring domains: the LCP acts as its own 'matrix'. Due to the lower $T_{g}$ of PLLA compared to the LCPs, the LCP in the LCP-PLLA tapes experiences a lower matrix viscosity compared to the pure LCP tapes. Initially, there is difference between the behavior of the LCP-PLA tapes and the LCP-PLLA tapes. This is in agreement with the concept that the matrix viscosity determines the onset of relaxation, as the molecular weight and viscosity of both PLA's are similar. At higher temperatures, the crystallites formed in the semi-crystalline PLLA prevent relaxation of the LCP's fibrillar morphology and delay the decrease in the interchain orientation with respect to the LCP fibrils in amorphous PLA. The molecular relaxation of the LCP in the semi-crystalline matrix resembles that of the pure LCP, governed by contraction of the polydomain texture followed by coalescence, as it shows a similar slow onset and eventually maintains a constant degree of orientation.

In the tapes with a semi-crystalline PLLA matrix, the temperature to which the tapes are heated affects the relaxation significantly. As previously seen, melting of the PLLA crystals allows the relaxation of the LCP's fibrillar structure, correspondingly diminishing the degree of orientation. In the case the tapes are heated to a temperature below the melting temperature of PLLA, a level of orientation of the LCP chains is maintained and this level depends on the annealing temperature. When annealed at 170 ${ }^{\circ} \mathrm{C}$ both CE-2:1 and CE-1:1 retain a higher degree of orientation compared to when the respective tapes are annealed at $175^{\circ} \mathrm{C}$. 

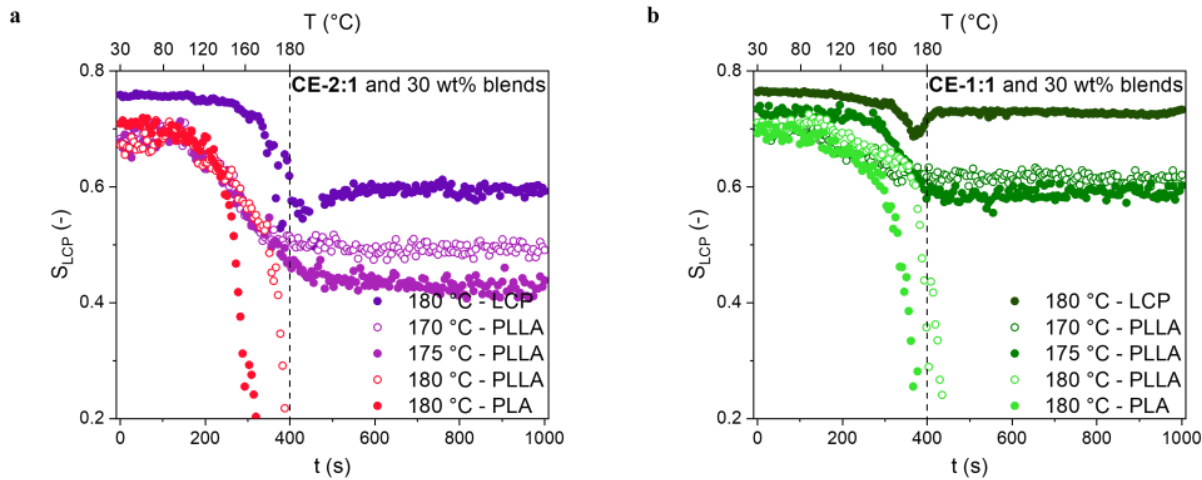

Figure 6.8 (a) Comparison of the evolution of $S_{L C P}$ for different types of CE-2:1 tapes upon heating. (b) Comparison of the evolution of $S_{L C P}$ for different types of CE-1:1 tapes upon heating.

\section{Cold-crystallization of PLLA in the presence of LCP fibrils}

The effect of the matrix phase on the relaxation of the LCP has been described in detail in previous sections. However, it is known that LCPs can also affect the crystallization behavior of the matrix. For the produced LCP-PLLA tapes, a nucleation effect of the LCP's surface (eg. some form of epitaxy) is unlikely, as the cold crystallization upon heating of the tapes is not notably accelerated in the presence of the LCPs. In fact, the crystallization of the PLLA is hindered in the presence of any of the LCPs during subsequent cooling, as shown in Figure S6.6 of the Supporting Information. The PLLA matrix appears isotropic prior to heating, however upon crystallization during heating (cold-crystallization) we observe the rise of an interchain diffraction signal with a orientation along the drawing direction $\left(90^{\circ}\right.$ and $\left.270^{\circ}\right)$. Due to the small thickness and width of the tape a confinement effect is expected to facilitate dominant growth of lamellae along the drawing direction ${ }^{36,37}$. However, as shown in Figure 6.9a, the cold crystallization of the PLLA is affected by the presence of the CE1:1 fibrils. Upon heating, the tapes containing LCP fibrils and an apparently isotropic PLLA matrix, crystallize and the 110-reflection of the PLLA crystals exhibits a 6-fold symmetry. It is hypothesized that the LCP fibrils limit the previously observed dominant growth of the PLLA lamellae along the drawing direction. Additionally, we consider it likely that the presence of the LCP induces stresses in the PLLA, both during processing and during heating of the tapes. Such (residual) stresses are known to induce the growth of twisted lamellae in materials such as $i$-PP and PLLA ${ }^{38-41}$, which is a plausible explanation for the reflections at $60^{\circ}$ and $120^{\circ}$ (and correspondingly at $240^{\circ}$ and $300^{\circ}$ ). The signals become more dominant in the tapes containing the more viscous LCPs (CE-3:2 and CE-1:1, Figure 6.9b) since these require more stress to deform and therefore result in more residual stresses. In the LCP-PLLA tapes, a signal arises where 
the PLLA chains within the crystall are oriented along the drawing direction $\left(0^{\circ}\right.$ and $180^{\circ}$ ), which are more dominant in the tapes containing the lower viscous LCPs $(\mathbf{L C P P}$ and CE-2:1). A full overview of the diffraction patterns of the annealed tapes is displayed in Figure S6.7 of the Supporting Information.

a

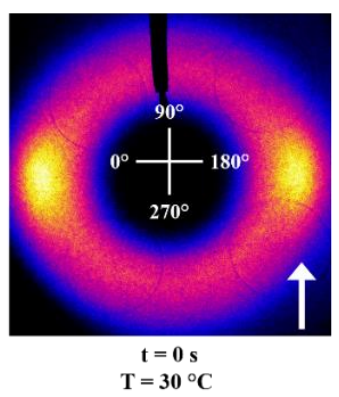

b

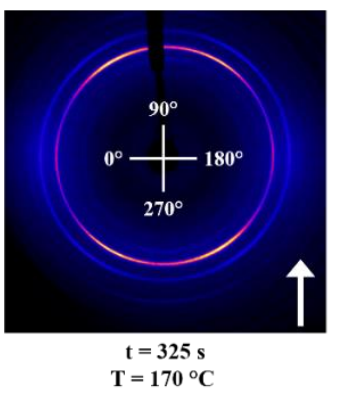

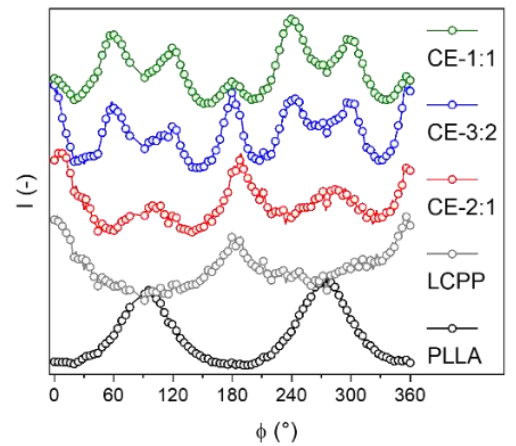

Figure 6.9 (a) Diffraction patterns of CE-1:1-PLLA tapes at $30^{\circ} \mathrm{C}$ and at $170{ }^{\circ} \mathrm{C}$. The white arrows mark the drawing direction; the angles displayed at the center mark the azimuthal angles as displayed in Figure 6.9b. (b) Normalized azimuthal intensity of the PLLA 110-reflection in the PLLA tape and the different LCP-PLLA tapes, measured at $170{ }^{\circ} \mathrm{C}$. Azimuthal angles of $90^{\circ}$ and $270^{\circ}$ correspond to the drawing direction.

\subsection{Conclusions}

We have demonstrated the production of reinforced thermoplastic composites based on several LCPs with different molar masses and PLLA. The morphology of the injection molded composites is evaluated and is found to be in qualitative agreement with predictions based on theory and the viscoelastic behavior of the blend constituents. This highlights not only the enormous influence the morphology and the mechanical properties of the reinforcing phase have on the overall performance of the composites, but also the potential benefit of LCPs with tunable molecular weight and viscosity. A fibrillar morphology is obtained via injection molding for all composites, even forming fibrils with sub-micron diameters in the case of the LCPs with lower molar mass. Though a highly suitable morphology is formed for all the tested LCPs, the mechanical performance of the composites containing LCPs with higher molar mass improved more substantially. This more significant increase is attributed to the intrinsic mechanical performance of these chain extended LCPs. Compared to the pure PLLA samples, the Young's modulus and stress at break of the injection molded composites containing the high molar mass LCP is increased by $60 \%$ and $40 \%$, respectively. Interestingly, this does not result in increased brittleness of the composite, due to a high 
interfacial strength and the relatively high ductility of the used LCP. The reprocessability of the LCP-PLLA composites is demonstrated.

The relaxation of the interchain orientation of the LCP in a PLA matrix is evaluated in detail. The viscosity of the matrix-phase is found to be the dominant parameter. In blends, the mechanism by which the interchain orientation of the LCP decreases differs from pure LCPs as it is related to the relaxation of the fibrillar LCP morphology; The orientation of the LCP is maintained when the fibrillar LCP morphology remains intact, eg. when the matrix cold crystallizes upon heating.

The presence of the LCP is found to affect the cold crystallization of the PLLA matrix. Although, no increase in nucleation or crystallization rate is observed, the PLLA crystallites were oriented not only along the draw direction but also at angles of $60^{\circ}$ and $120^{\circ}$ with respect to the draw direction. The relative intensity of the signal at $60^{\circ}$ and $120^{\circ}$ to the draw direction increases with the molar mass of the dispersed LCP and are the result of the twisted lamellae formed due to residual stresses. Overall, these findings highlight the importance of viscosity control in thermoplastic blends; control over both the matrix and filler viscosity and their temperature dependency allow for control over particle size, distribution, relaxation, interchain orientation and lamellar morphology and orientation upon cold-crystallization of the matrix. The resulting composites do not only possess good mechanical properties, but are also reprocessable. 


\subsection{References}

(1) Bakis, C. E.; Bank, L. C.; Brown, V. L.; Cosenza, E.; Davalos, J. F.; Lesko, J. J.; Machida, A.; Rizkalla, S. H.; Triantafillou, T. C. Fiber-Reinforced Polymer Composites for Construction - State-of-the-Art Review. J. Compos. Constr. 2002, 6 (2), 73-87. https://doi.org/10.1061/(ASCE)1090-0268(2002)6:2(73).

(2) Asmatulu, E.; Twomey, J.; Overcash, M. Recycling of Fiber-Reinforced Composites and Direct Structural Composite Recycling Concept. J. Compos. Mater. 2014, 48 (5), 593-608. https://doi.org/10.1177/0021998313476325.

(3) Song, Y. S.; Youn, J. R.; Gutowski, T. G. Life Cycle Energy Analysis of Fiber-Reinforced Composites. Compos. Part A Appl. Sci. Manuf. 2009, 40 (8), 1257-1265. https://doi.org/10.1016/j.compositesa.2009.05.020.

(4) Pimenta, S.; Pinho, S. T. Recycling Carbon Fibre Reinforced Polymers for Structural Applications: Technology Review and Market Outlook. Waste Manag. 2011, 31 (2), 378 392. https://doi.org/10.1016/j.wasman.2010.09.019.

(5) Eriksson, P. A.; Albertsson, A. C.; Boydell, P.; Prautzsch, G.; Månson, J. A. E. Prediction of Mechanical Properties of Recycled Fiberglass Reinforced Polyamide 66. Polym. Compos. 1996, 17 (6), 830-839. https://doi.org/10.1002/pc.10676.

(6) Kuram, E.; Tasci, E.; Altan, A. I.; Medar, M. M.; Yilmaz, F.; Ozcelik, B. Investigating the Effects of Recycling Number and Injection Parameters on the Mechanical Properties of Glass-Fibre Reinforced Nylon 6 Using Taguchi Method. Mater. Des. 2013, 49, 139-150. https://doi.org/10.1016/j.matdes.2013.02.027.

(7) Davies, P.; Le Duigou, A.; Pillin, I.; Baley, C.; Bourmaud, A. Effect of Recycling on Mechanical Behaviour of Biocompostable Flax/Poly(l-Lactide) Composites. Compos. Part A Appl. Sci. Manuf. 2008, 39 (9), 1471-1478. https://doi.org/10.1016/j.compositesa.2008.05.008.

(8) Thomason, J. L.; Vlug, M. A.; Schipper, G.; Krikor, H. G. L. T. Influence of Fibre Length and Concentration on the Properties of Glass Fibre-Reinforced Polypropylene: Part 3. Strength and Strain at Failure. Compos. Part A Appl. Sci. Manuf. 1996, 27 (11), 1075-1084. https://doi.org/10.1016/1359-835X(96)00066-8.

(9) Morin, C.; Loppinet-Serani, A.; Cansell, F.; Aymonier, C. Near- and Supercritical Solvolysis of Carbon Fibre Reinforced Polymers (CFRPs) for Recycling Carbon Fibers as a Valuable Resource: State of the Art. J. Supercrit. Fluids 2012, 66, 232-240. https://doi.org/10.1016/j.supflu.2012.02.001.

(10) Goto, M.; Sasaki, M.; Tokuno, S.; Shibata, K.; Iwaya, T. Recycling of Fiber Reinforced Plastics Using Depolymerization by Solvothermal Reaction with Catalyst. J. Mater. Sci. 2007, 43 (7), 2452-2456. https://doi.org/10.1007/s10853-007-2017-8.

(11) Bassett, B. R.; Yee, A. F. A Method of Forming Composite Structures Using in Situ Formed Liquid Crystal Polymer Fibers in a Thermoplastic Matrix. Polym. Compos. 1990, 11 (1), 10-18. https://doi.org/10.1002/pc.750110103.

(12) Wang, H.; Lee, K. W.; Chung, T. S.; Jaffe, M. Rheology, Morphology and Properties of LCP/Nylon 66 Composite Fibers. Polym. Compos. 2000, 21 (1), 114-123. https://doi.org/10.1002/pc.10170.

(13) Kiss, G. In Situ Composites: Blends of Isotropic Polymers and Thermotropic Liquid Crystalline Polymers. Polym. Eng. Sci. 1987, 27 (6), 410-423. https://doi.org/10.1002/pen.760270606.

(14) Blizard, K. G.; Federici, C.; Federico, O.; Chapoy, L. L.; Caduti, V. The Morphology of Extruded Blends Containing a Thermotropic Liquid Crystalline Polymer. Polym. Eng. Sci. 1990, 30 (22), 1442-1453. https://doi.org/10.1002/pen.760302205. 
(15) Silverstein, M. S.; Hiltner, A.; Baer, E. Hierarchical Structure in LCP/PET Blends. J. Appl. Polym. Sci. 1991, 43 (1), 157-173. https://doi.org/10.1002/app.1991.070430113.

(16) Chung, T.-S. The Recent Developments of Thermotropic Liquid Crystalline Polymers. Polym. Eng. Sci. 1986, 26 (13), 901-919. https://doi.org/10.1002/pen.760261302.

(17) Han, H.; Bhowmik, P. K. Wholly Aromatic Liquid-Crystalline Polyesters. Prog. Polym. Sci. 1997, 22 (7), 1431-1502. https://doi.org/10.1016/S0079-6700(96)00028-7.

(18) Heino, M. T.; Hietaoja, P. T.; Vainio, T. P.; Seppälä, J. V. Effect of Viscosity Ratio and Processing Conditions on the Morphology of Blends of Liquid Crystalline Polymer and Polypropylene. J. Appl. Polym. Sci. 1994, $51 \quad$ (2), 259-270. https://doi.org/10.1002/app.1994.070510208.

(19) Tan, L. P.; Yue, C. Y.; Tam, K. C.; Lam, Y. C.; Hu, X. Effects of Shear Rate, Viscosity Ratio and Liquid Crystalline Polymer Content on Morphological and Mechanical Properties of Polycarbonate and LCP Blends. Polym. Int. 2002, 51 (5), 398-405. https://doi.org/10.1002/pi.889.

(20) Bras, W.; Dolbnya, I. P.; Detollenaere, D.; Van Tol, R.; Malfois, M.; Greaves, G. N.; Ryan, A. J.; Heeley, E. Recent Experiments on a Combined Small-Angle/Wide-Angle X-Ray Scattering Beam Line at the ESRF. J. Appl. Crystallogr. 2003, 36 (3 I), 791-794. https://doi.org/10.1107/S002188980300400X.

(21) Portale, G.; Cavallo, D.; Alfonso, G. C.; Hermida-Merino, D.; Van Drongelen, M.; Balzano, L.; Peters, G. W. M.; Goossens, J. G. P.; Bras, W. Polymer Crystallization Studies under Processing-Relevant Conditions at the SAXS/WAXS DUBBLE Beamline at the ESRF. J. Appl. Crystallogr. 2013, 46 (6), 1681-1689. https://doi.org/10.1107/S0021889813027076.

(22) Mitchell, G. R.; Windle, A. H. Orientation in Liquid Crystal Polymers. In Developments in crystalline polymers; Basset, D. C., Ed.; Elsevier Applied Science: London, UK, 1988. https://doi.org/10.1007/978-94-009-1341-7_3.

(23) de Kort, G. W.; Rastogi, S.; Wilsens, C. H. R. M. Controlling Processing, Morphology, and Mechanical Performance in Blends of Polylactide and Thermotropic Polyesters. Macromolecules 2019, 52, 6005-6017. https://doi.org/10.1021/acs.macromol.9b01083.

(24) Shi, H.; Utracki, L. A. Development of Polymer Blend Morphology During Compounding in a Twin-Screw Extruder. Part II : Theoretical Derivations. Polym. Eng. Sci. 1992, 32 (24), 1834-1845. https://doi.org/10.1002/pen.760322406.

(25) de Kort, G. W.; Bouvrie, L.; Rastogi, S.; Wilsens, C. H. R. M. Thermoplastic PLA-LCP Composites: A Route towards Sustainable, Reprocessable, and Recyclable Reinforced Materials. Sustain. Chem. Eng. 2019, 1-35. https://doi.org/10.1021/acssuschemeng.9b06305.

(26) Sigillo, I.; Grizzuti, N.; Tecchio, I.; Ii, F. The Effect of Molecular Weight on the Steady Shear Rheology of Lyotropic Solutions . A Phenomenological Study. 1994, 38 (June), 589-599. https://doi.org/10.1122/1.550476.

(27) Somma, E.; Nobile, M. R. The Linear Viscoelastic Behavior of a Series of Molecular Weights of the Thermotropic Main-Chain Liquid Crystal Polymers HBA/HNA 73/27. J. Rheol. (N. Y. N. Y). 2004, 48 (6), 1407-1423. https://doi.org/10.1122/1.1795194.

(28) de Bruijn, R. A. Deformation and Breakup of Drops in Simple Shear Flows, Eindhoven University of Technology, 1989. https://doi.org/10.6100/IR318702.

(29) Kernick, W. a.; Wagner, N. J. The Role of Liquid-Crystalline Polymer Rheology on the Evolving Morphology of Immiscible Blends Containing Liquid-Crystalline Polymers. J. Rheol. (N. Y. N. Y). 1999, 43 (3), 521. https://doi.org/10.1122/1.551038. 
(30) Kantz, M. R. The Effects of Melt Processing Variables on the Morphology and Properties of Injection Molded Polypropylene. Int. J. Polym. Mater. Polym. Biomater. 1974, 3 (3), 245-258. https://doi.org/10.1080/00914037408072355.

(31) Roozemond, P. C.; Van Drongelen, M.; Ma, Z.; Spoelstra, A. B.; Hermida-Merino, D.; Peters, G. W. M. Self-Regulation in Flow-Induced Structure Formation of Polypropylene. Macromol. Rapid Commun. 2015, 36 (4), 385-390. https://doi.org/10.1002/marc.201400505.

(32) Li, Z. M.; Yang, W.; Yang, S.; Huang, R.; Yang, M. B. Morphology-Tensile Behavior Relationship in Injection Molded Poly(Ethylene Terephthalate)/Polyethylene and Polycarbonate/Polyethylene Blends (I): Part I Skin-Core Structure. J. Mater. Sci. 2004, 39 (2), 413-431. https://doi.org/10.1023/B:JMSC.0000011494.19843.26.

(33) Turcott, E.; Nguyen, K. T.; Garcia-Rejon, A. Microstructure Development during the Injection Molding of PET/LCP Blends. Polym. Eng. Sci. 2001, 41 (4), 603-617. https://doi.org/10.1002/pen.10757.

(34) Hsiao, B. S.; Stein, R. S.; Deutscher, K.; Winter, H. H. Optical Anisotropy of a Thermotropic Liquid- crystalline Polymer in Transient Shear. J. Polym. Sci. Part B Polym. Phys. 1990, 28 (9), 1571-1588. https://doi.org/10.1002/polb.1990.090280912.

(35) de Kort, G. W.; Leoné, N.; Stellamanns, E.; Auhl, D.; Wilsens, C. H. R. M.; Rastogi, S. Effect of Shear Rate on the Orientation and Relaxation of a Vanillic Acid Based Liquid Crystalline Polymer. Polymers (Basel). 2018, $10 \quad$ (9), 935. https://doi.org/10.3390/polym10090935.

(36) Woo, E. M.; Lugito, G.; Tsai, J. H. Effects of Top Confinement and Diluents on Morphology in Crystallization of Poly(1 -Lactic Acid) Interacting with Poly(Ethylene Oxide). J. Polym. Sci. Part B Polym. Phys. 2015, 53 (16), 1160-1170. https://doi.org/10.1002/polb.23756.

(37) Fischer, F. S. U.; Tremel, K.; Sommer, M.; Crossland, E. J. C.; Ludwigs, S. Directed Crystallization of Poly(3-Hexylthiophene) in Micrometre Channels under Confinement and in Electric Fields. Nanoscale 2012, 4 (6), 2138-2144. https://doi.org/10.1039/c2nr12037b.

(38) Lohmeijer, P. J. A.; Goossens, J. G. P.; Peters, G. W. M. Quiescent Crystallization of Poly(Lactic Acid) Studied by Optical Microscopy and Light-Scattering Techniques. J. Appl. Polym. Sci. 2017, 134 (10), 2-10. https://doi.org/10.1002/app.44566.

(39) Lotz, B.; Cheng, S. Z. D. A Critical Assessment of Unbalanced Surface Stresses as the Mechanical Origin of Twisting and Scrolling of Polymer Crystals. Polymer (Guildf). 2005, 46 (3), 577-610. https://doi.org/10.1016/j.polymer.2004.07.042.

(40) Assouline, E.; Wachtel, E.; Grigull, S.; Lustiger, A.; Wagner, H. D.; Marom, G. Lamellar Twisting in $\alpha$ Isotactic Polypropylene Transcrystallinity Investigated by Synchrotron Microbeam X-Ray Diffraction. Polymer (Guildf). 2001, 42 (14), 6231-6237. https://doi.org/10.1016/S0032-3861(01)00087-8.

(41) Maillard, D.; Prud'homme, R. E. Crystallization of Ultrathin Films of Polylactides: From Chain Chirality to Lamella Curvature and Twisting. Macromolecules 2008, 41 (5), 17051712. https://doi.org/10.1021/ma071306u. 


\subsection{Supporting Information}

\section{Evaluation of the linear viscoelastic regime for the LCPs at different temperatures}

To ensure an accurate comparison between the viscoelastic behavior of the different LCPs, the viscosity of LCPP and CE-1:1 were measured as function of the applied strain at $150{ }^{\circ} \mathrm{C}$ and $190^{\circ} \mathrm{C}$ (Figure S6.1). Both LCPs show a constant viscosity at strains below $10 \%$.

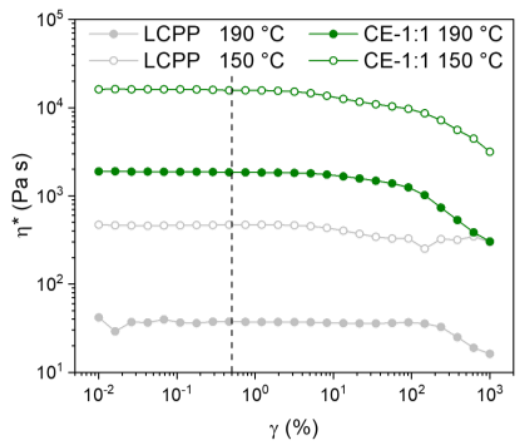

Figure S6.1 The complex viscosity of LCPP and CE-2:1 at $190^{\circ} \mathrm{C}$ and $150^{\circ} \mathrm{C}$ as a function of oscillation $\operatorname{strain}\left(\omega=\mathrm{rad} \mathrm{s}^{-1}\right)$. The strain at which the frequency sweeps were measured is marked by the dotted line.

Fibril morphology of injection molded bars containing $30 \mathrm{wt} \%$ of the respective LCPs
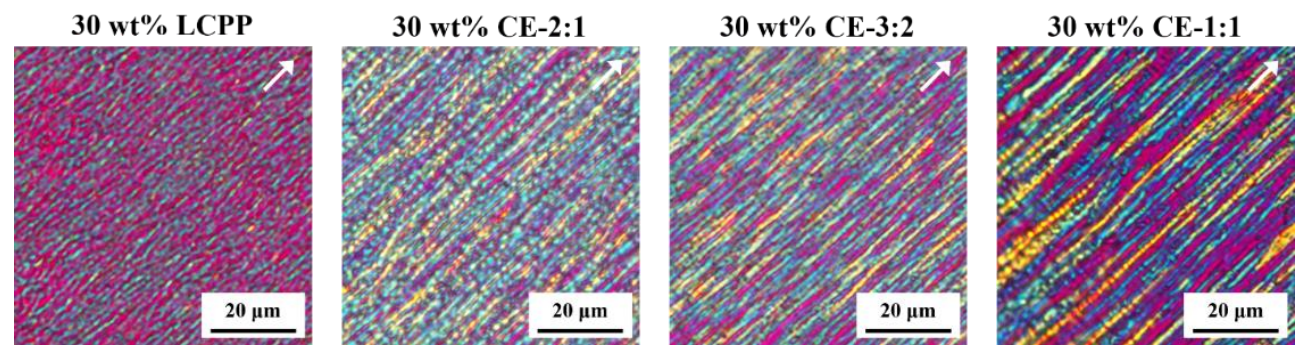

Figure S6.2 Morphology of injection molded composites containing the different LCPs. The composites were microtomed to slices with a thickness of $2.5 \mu \mathrm{m}$. The images represent the core-region of the sample. The white arrows mark the injection direction.

All composites have a fine fibrillar morphology. The LCP particles, although clearly deformed, appeared short in the images taken from the microtomed slices with a thickness of $1 \mu \mathrm{m}$. To show that actual fibrils are formed in the injection molding process, POM images obtained from microtomed slices with a thickness of $2.5 \mu \mathrm{m}$ are displayed here. In all composites, fibrils with lengths over $100 \mu \mathrm{m}$ are observed, and the 
observed trends with respect to the fibril diameter and appearance are in agreement with those in the main paper.

\section{Layer morphology of injection molded bars containing $30 \mathrm{wt} \%$ of the} respective $\mathrm{LCPs}$
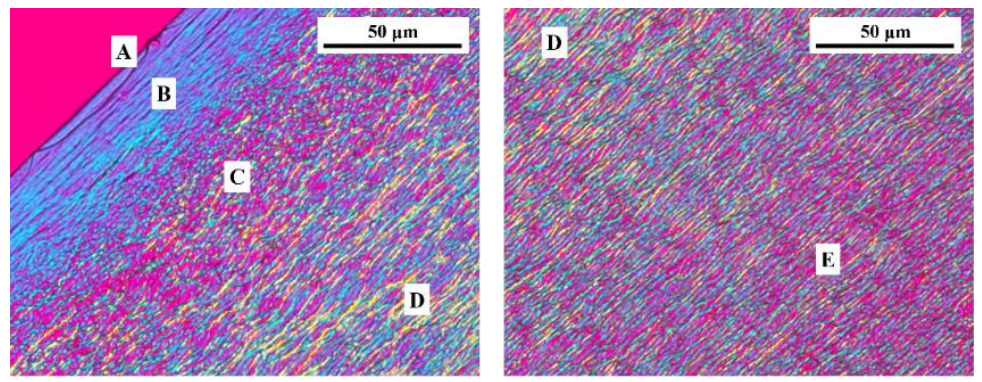

$A=$ quench layer

$B=$ shear layer

$\mathrm{C}=$ transition layer $\mathbf{I}$

D = transition layer II

$\mathbf{E}=$ core
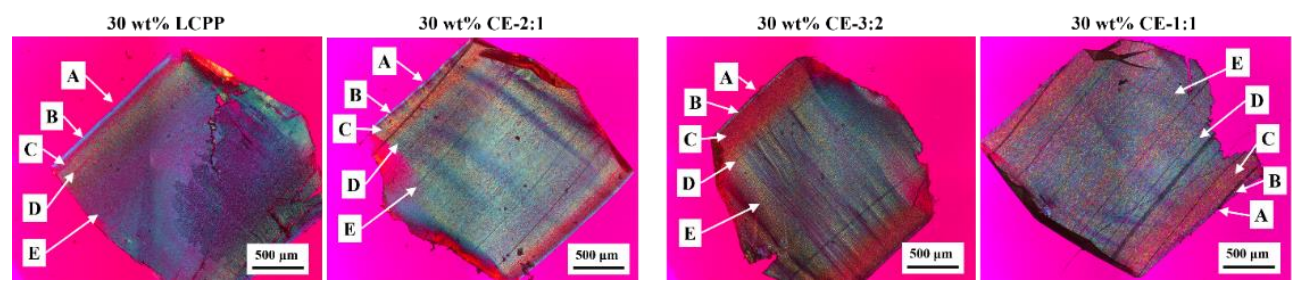

Figure S6.3 (top) polarized optical microscopy images of the morphology the $30 \mathrm{wt} \%$ LCPP/PLLA composite. The different layers that are observed are labeled $(A-E)$. Images were obtained from microtomed slices with a thickness of $2.5 \mu \mathrm{m}$. (bottom) Cross-sections of the LCP - PLLA composites. The different layers that are observed are labeled (A-E). Images were obtained from microtomed slices with a thickness of $2.5 \mu \mathrm{m}$.

Injection molded parts often display a layered morphology, caused by differences in the deformation rate and cooling rate throughout the cross-section of the part. In the case of an LCP dispersed in a thermoplastic matrix, this leads to differences in the LCP morphology. Five distinct layers are observed in the LCP-PLLA blends, as shown in Figure S6.3: 1) a quench layer in direct contact with the wall, 2) a shear layer close to the wall, 3) a transition layer a bit further from the wall, 4) a second transition layer, 5) a core region at the center of the sample. In the quenched layer, the material is cooled extremely quickly by the wall, preventing the formation of oriented structures. This layer is in the order of $1 \mu \mathrm{m}$. In the shear layer, the material experiences high stresses but slightly lower cooling rates and highly oriented LCP platelets are formed. The first transition layer characteristically has shorter, irregular fibrils, as the stresses are reduced at this position but the cooling rates remain relatively high. The second transition layer and core layer both have a fibrillar morphology. The fibrils in the second transition layer are thicker and longer. In both these layers, the cooling rate is relatively low, due to thermal insulation by the other layers. The higher stress in the transition layer allows the formation of longer fibrils. 
The effective viscosity ( $\eta_{\text {eff }}$ ) of a molten blend is affected by the presence of the different LCPs: a more viscous LCP results in a higher $\eta_{\text {eff. }}$ The implications are shown in Figure S6.4.

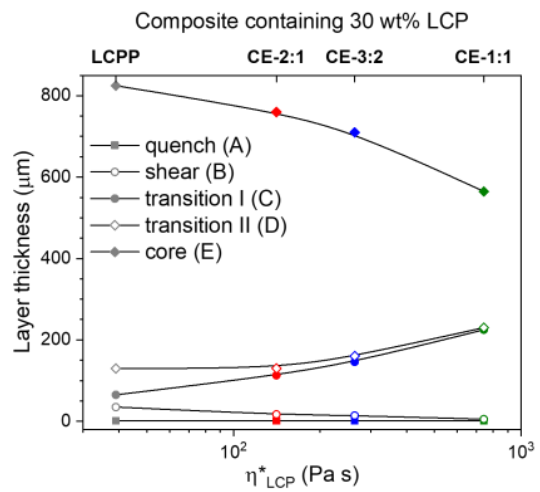

Figure S6.4 Trends in the thickness of the different layers as a function of the LCP viscosity as observed from the mircotomed cross-sections of the different LCP-PLLA composites. The viscosity of the different LCPs (measured in parallel plate rheology at $\omega=137 \mathrm{rad} \mathrm{s}^{-1}, \mathrm{~T}=190^{\circ} \mathrm{C}$ ).

\section{Comparison of the viscoelastic behavior of semi-crystalline PLLA and amorphous PLA}

In this study, melt drawn tapes containing of $30 \mathrm{wt} \%$ of the respective LCPs are used to investigate the relaxation behavior of the LCPs in a matrix. Two different grades are used: a semi-crystalline PLLA (low D-isomer content), and an amorphous PLA (higher D-isomer content). For fair comparison, the molecular weight and the viscoelastic behavior of both grades needs to be comparable. This is confirmed via plate-plate rheology, (Figure S6.5).

a

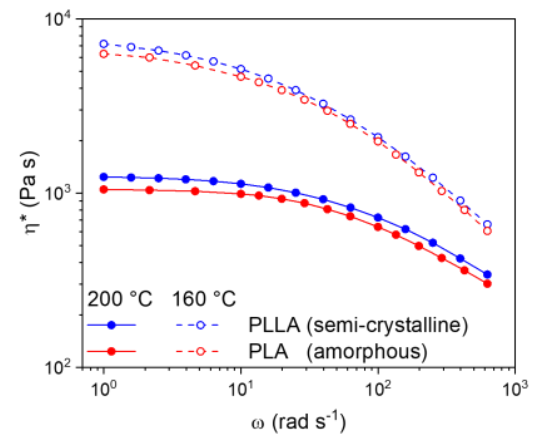

b

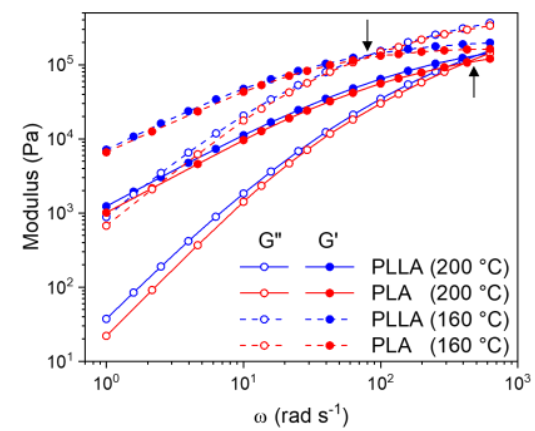

Figure S6.5 (a) Complex viscosity as a function of oscillation frequency of the semi-crystalline PLLA and amorphous PLA. (b) Viscous modulus and elastic modulus as a function of oscillation frequency of the semicrystalline PLLA and amorphous PLA. The black arrows mark the respective crossover points. 


\section{DSC traces of melt drawn tapes}

The thermal behavior of the melt drawn tapes is evaluated by differential scanning calorimetry using a TA Instruments Q2000 DSC. The heating and cooling-rates of the sample were $10{ }^{\circ} \mathrm{C} \mathrm{min}^{-1}$ and measurements were performed under a nitrogen rich atmosphere. A significant enthalpy relaxation is observed on the $T_{g}$ of PLLA (Figure S6.6a) and PLA (Figure S6.6c), and the respective $T_{g}$ 's shift to slightly higher values for the composite tapes. The cold crystallization of the PLLA occurs at a comparable temperature, regardless of the presence of the LCPs, whereas the melting temperature is slightly reduced due to the LCPs. The enthalpy corresponding to the cold crystallization and melting of the PLLA is reduced according to the weight fraction of LCP present.

a

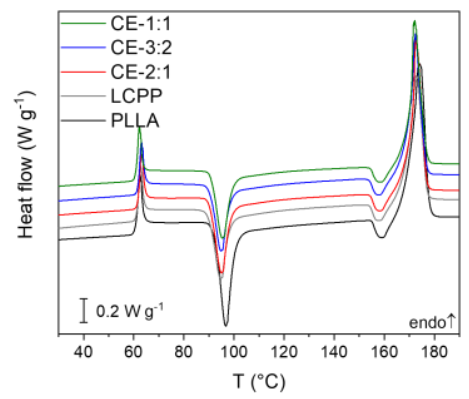

c

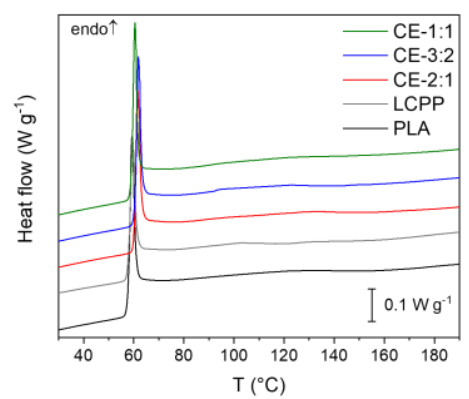

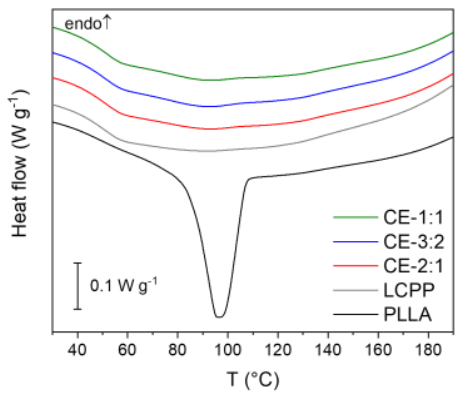

d

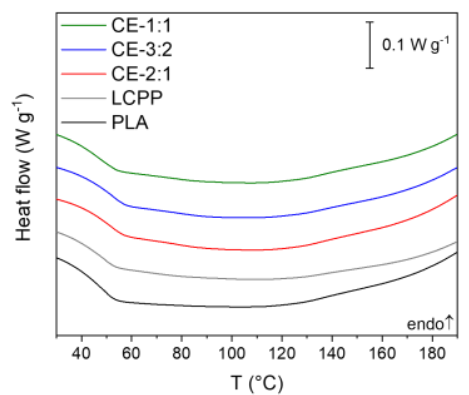

Figure 6.6 (a) First heating trace of melt drawn tapes containing semi-crystalline PLLA and the LCPs (10 $\left.{ }^{\circ} \mathrm{C} \mathrm{min}{ }^{-1}\right)$. (b) First cooling trace of melt drawn tapes containing semi-crystalline PLLA and the LCPs $\left(10^{\circ} \mathrm{C}\right.$ min $\left.^{-1}\right)$. (c) First heating trace of melt drawn tapes containing amorphous PLA and the LCPs $\left(10^{\circ} \mathrm{C} \mathrm{min}{ }^{-1}\right)$. (d) First cooling trace of melt drawn tapes containing amorphous PLA and the LCPs $\left(10^{\circ} \mathrm{C} \mathrm{min}{ }^{-1}\right)$.

Upon cooling at a rate of $10{ }^{\circ} \mathrm{C} \mathrm{min}^{-1}$, the pure PLLA tape has a crystallization peak at $96{ }^{\circ} \mathrm{C}$, whereas crystallization does not occur in the tapes containing LCPs (Figure $\mathrm{S} 6.6 \mathrm{~b})$. In the composite tapes, the $T_{g}$ 's of the dispersed LCP phase $\left(\sim 94^{\circ} \mathrm{C}\right)$ and PLLA matrix phase $\left(\sim 55^{\circ} \mathrm{C}\right)$ are clearly observed. Neither cold crystallization upon heating 
(Figure S6.6c), nor crystallization upon cooling (Figures S6.6d) are observed in the melt drawn PLA tape or composites with the amorphous PLA as a matrix. The $T_{g}$ 's of the respective LCPs and PLA are visible in each trace, and comparable to those of the melt drawn tapes with the PLLA matrix.

\section{D-WAXD diffraction patterns of melt drawn tapes with PLLA}

The WAXD diffraction patterns of the melt drawn tapes show an isotropic PLLA matrix phase (diffuse ring at lower $\mathrm{q}$ ) and an oriented LCP phase (diffuse arc at higher q) prior to heating (Figure S6.7, Top). Upon heating, cold crystallization of the PLLA occurs. Though the PLLA appears isotropic, oriented crystals are formed and the orientation is effected by the different LCPs (Figure S6.7, Middle). In the tapes containing LCPs the azimuthal intensity of the PLLA crystals shows a 6 -fold symmetry, with signal corresponding to $0^{\circ}, 60^{\circ}$ and $120^{\circ}$. The reflection at $0^{\circ}$ corresponds to chains oriented according to the drawing direction and is more dominant in the tapes containing the LCPs with low molecular weights (LCPP and CE-2:1). The signals at $60^{\circ}$ and $120^{\circ}$ become stronger at high LCPs molecular weight, due to twisted lamellae formed by residual stresses. The LCP orientation is largely maintained as the tapes are heated to $170{ }^{\circ} \mathrm{C}$, as the diffuse scattering arcs remain (Figure S6.7, Bottom). The signal intensity of the oriented LCPs is low due to the diffraction due to the PLLA crystallites.

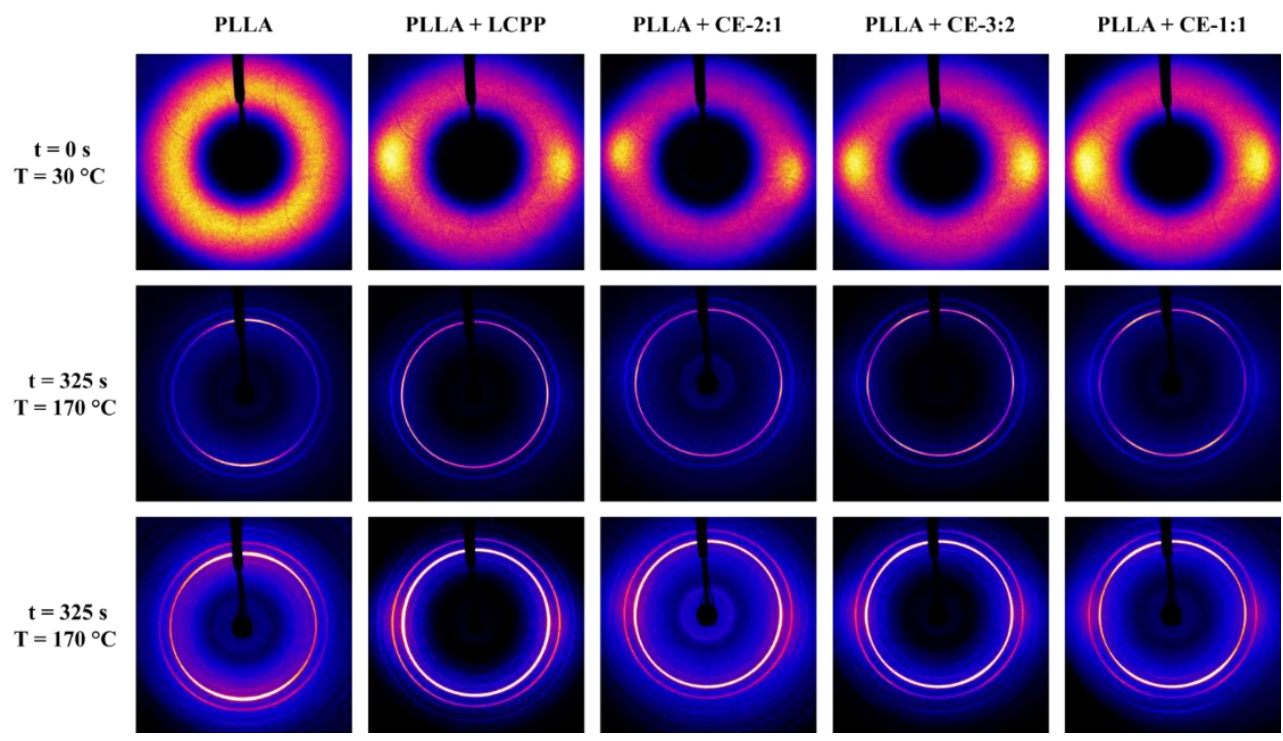

Figure S6.7 (Top) Diffraction patterns of the melt drawn LCP-PLLA tapes and the pure PLLA tapes, as measured prior to heating. (Middle) Diffraction pattern of the same tapes upon heating to $170{ }^{\circ} \mathrm{C}$, the intensity is set to highlight the signal corresponding to the PLLA crystals. (Bottom) Diffraction pattern of the same tapes upon heating to $170{ }^{\circ} \mathrm{C}$, the intensity is set show the remaining orientation of the LCP phase. 


\section{Chapter 7 - Valorization addendum}

The previous chapters of this thesis describe my findings over the past four years regarding the development of reprocessable reinforced thermoplastic composites based on liquid crystalline polymers and poly(lactide) with the underlying goal to boost the development and implementation of sustainable plastic materials. Chapters 2 through 4 investigate the orientation and relaxation of LCPs, identify feasible processing routes and crucial LCP properties for the production of reinforced thermoplastic composites, demonstrate the reprocessability of LCP-PLA composites and identify the boundary conditions for their reprocessability. The viscosity of the two thermoplastic polymers, as well as their ratio and their respective temperature dependence are identified as the key parameters that determine the morphology and performance of the composite, given that the processing conditions are kept the same. These parameters need to remain constant over the course of multiple processing cycles to maintain the performance of the composites upon reprocessing. As the processing temperatures of typical LCPs are high and PLA is susceptible to thermal degradation, this can pose a challenge.

To address this issue a versatile method to produce LCPs with a low processing temperature has been developed, which is described in Chapter 5 and these LCPs are used to produce reinforced composites with PLA in Chapter 6. This method is based on the chain extension of a liquid crystalline prepolymer with carboxylic end-groups with bis(2-oxazoline)s, which allows control over the molecular weight and chain architecture of the final LCP. The backbone of the prepolymer consists of several different aromatic monomers, including bent and substituted monomers, some of which were biobased, that are incorporated randomly into the chains. This results in nematic glasses with excellent mechanical performance and processing temperatures below $190{ }^{\circ} \mathrm{C}$, which corresponds to the minimum processing temperature of semicrystalline PLLA. The thermal degradation of the PLLA upon reprocessing is therefore minimized. The composites based on PLLA and the chain extended LCPs possess good mechanical properties, comparable those of short glass fiber-reinforced thermoplastic polymers and proved to be reprocessable. This similarity provides a clear range of applications where these composites can potentially be used. As we consider these materials a viable and more sustainable alternative to other reinforced composites, a patent application has been filed on chain extended thermotropic LCPs with a low processing temperature and their in use thermoplastic composites. 


\subsection{Potential and challenges regarding industrial implementation of LCP reinforced thermoplastic composites}

From a material science point of view, chain extended LCPs are highly suitable to produce reprocessable reinforced composites with PLA or other thermoplastic materials. However, that alone is not sufficient to make the transition from a scientific study to actual market implementation. In order to achieve that the material needs to fit an unmet need, open a new market, have superior properties, or achieve the same at a lower cost. Several factors that favor the implementation of reprocessable LCP reinforced thermoplastic composites include:

1. The materials meet a societal need for materials that combine sustainability and performance.

2. There is a clear market for these composites based on their mechanical performance.

3. The composites can be processed via commonly used techniques.

4. The properties of the materials can be tuned towards a specific application.

Depending on the product and processing route, however, changes in product design and mold design might be required to obtain products with the desired properties, as the LCP morphology is determined by the flow fields the molten blend experiences during processing. Overall, the industrial production and actual application of PLALCP composites seems feasible and these composites can be implemented easily to replace existing materials. However, additional factors, such as the economic viability of these composites and the technical feasibility of the recycling process, need to be addressed.

Typically, thermotropic mainchain polyesters are relatively expensive. As a result, these materials are used when outstanding thermal properties or mechanical performance is required, but this has limited the implementation of these materials in other applications. The high cost of these polymers is in part related to the monomers used (eg. 6-hydroxy-2-naphthoic acid, or 4,4'-dihydroxy biphenyl) and in part to the stringent synthesis conditions that are required. The liquid crystalline prepolymers that have been developed have the potential to overcome this challenge. The concept is based on the use of several different monomers to create a random backbone and suppress the processing temperature. Therefore, a wider range of monomers can be incorporated, circumventing the need for the use of certain expensive, aforementioned fossil-based monomers that are commonly used in commercial LCPs. The low 
processing temperature and molecular weight of the prepolymers limits the melt viscosity during the synthesis and allows for lower synthesis temperatures: the conditions required to produce the polymers are less stringent. These two factors make the liquid crystalline prepolymers relatively cheap to produce, at the very least in comparison to typical commercial LCPs.

In general, the recycling of plastic products faces one challenge: the material needs to be collected and separated effectively, with the additional difficulty that most products are not designed with recycling in mind. One of the main reasons for the inferior properties of thermomechanically-recycled plastics, in addition to the effects of degradation, is the contamination of the materials, either via the inclusion of different polymers or via the introduction of incompatible additives. Although the LCP-PLA composites are reprocessable, their properties are prone to change upon the introduction of contaminants. In order to tackle this challenge, not only for the materials studied in this thesis but for the recycling of plastics in general, a joined effort is required: polymer producers, product designers, plastic processors, recycling companies and regulatory bodies need to work together to develop products and materials that are both separable and recyclable in a an efficient manner. To this day, the lack of such a joined effort prevents the efficient recycling of plastics without deterioration of material properties and the implementation of recycled plastic materials into high quality products remains limited.

\subsection{Room for improvement: backbone composition and prepolymer}

\section{molar mass}

In this thesis, it has been demonstrated that chain extension of liquid crystalline prepolymers can be used to produce versatile LCPs with good mechanical performance. In turn, these LCPs can be used to produce reprocessable reinforced thermoplastic composites. However, in the main text of the thesis only a single prepolymer is discussed, whereas different combinations of chain architecture, chain composition and prepolymer molar mass are easily produced. To demonstrate the versatility of this method and to give an indication of the effect of the prepolymer molar mass on the final properties a set of prepolymers was synthesized (Figure 7.1). The chemical composition of the backbone differs from the prepolymer described in Chapter 5, the main differences being that no aromatic-aliphatic monomer is incorporated, and the amount of 4-hydroxy benzoic acid is higher. This composition was chosen to improve the mechanical performance of the LCPs, while maintaining the ability to be processed at low temperatures. Liquid crystalline prepolymers with molecular weights of 
$1 \mathrm{~kg} \mathrm{~mol}-1,5 \mathrm{~kg} \mathrm{~mol}^{-1}$ and $10 \mathrm{~kg} \mathrm{~mol}^{-1}$ were synthesized via the incorporation of decreasing amounts of isophthalic acid into the polymer backbone. Via a similar procedure as described in Chapter 5 , the chain extension reaction with a stoichiometric amount of $\mathbf{I a O x}$ was carried out via reactive extrusion and the obtained LCPs were injection molded into dogbones.
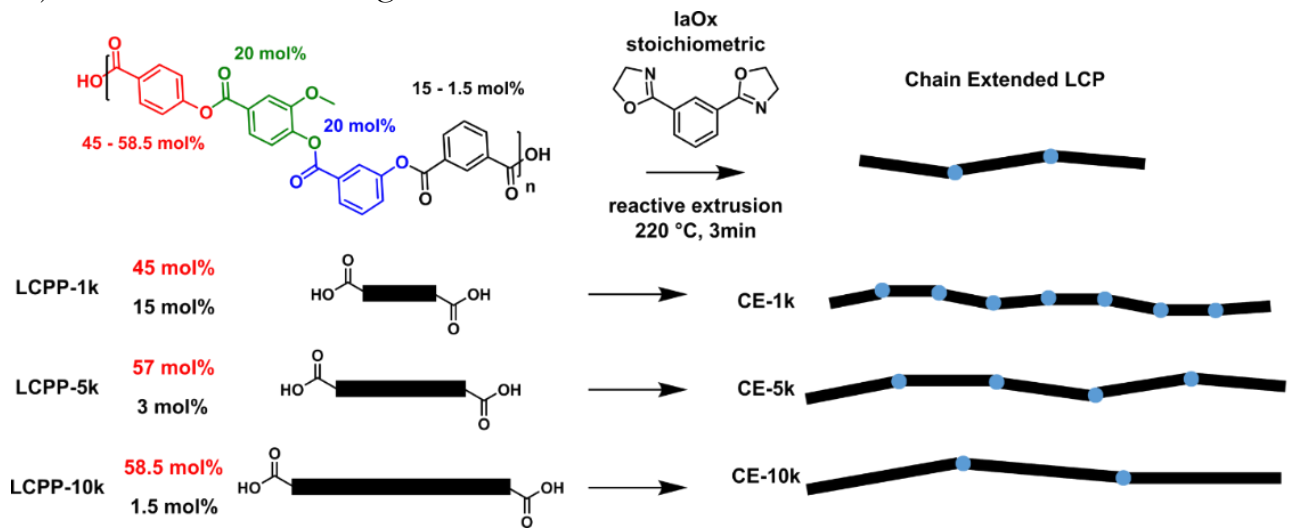

Figure 7.1 Chemical composition of the produced liquid crystalline prepolymers and schematic representation of their chains.

Increasing the molecular weight of the prepolymer has a positive effect on the tensile modulus of the chain extended LCP (Figure 7.2a). The chain extended LCPs that were obtained using a prepolymer with a molar mass of $10 \mathrm{~kg} \mathrm{~mol}^{-1}$ can reach a tensile modulus of $18.8 \mathrm{GPa}$. This value exceeds the stiffness obtained for any of the other LCPs described in this thesis, including commercially available thermotropic polyesters. The change in stiffness is not exclusively caused by the effect of prepolymer molecular weight, as there is a difference in the microstructure of the different LCPs (Figure 7.2b). Although both LCPs have an orientation along the injection direction, the sample based on the $1.0 \mathrm{~kg} \mathrm{~mol}^{-1}$ prepolymer is less oriented. Additionally, the increased amount of 4-hydroxy benzoic acid resulted in the formation of a small fraction of crystalline material. Despite the introduction of crystallites, these materials remain processable at low temperatures $\left(200-220{ }^{\circ} \mathrm{C}\right)$. These findings clearly demonstrate that further research into this type of LCP can provide versatile materials with even better mechanical properties. 


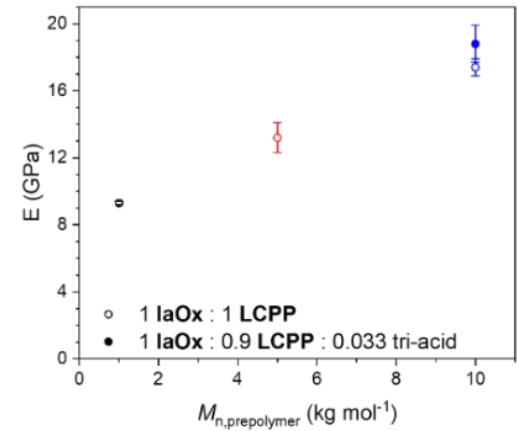

b

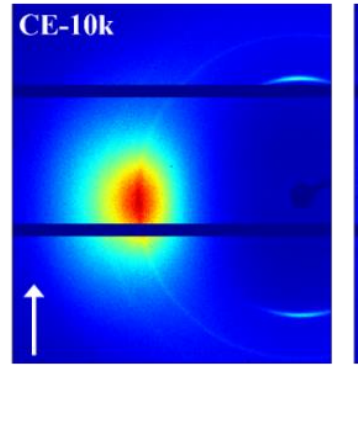

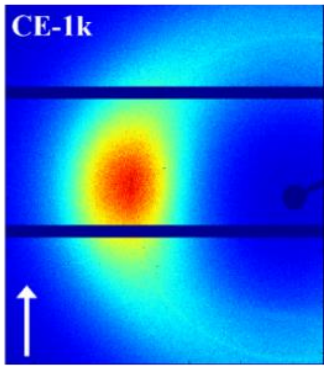

Figure 7.2 (a) Stiffness (E) as a function of prepolymer molecular weight. (b) 2D-WAXD patterns of injection molded samples consisting of chain extended LCPs with different prepolymer molecular weights.

\subsection{Towards different applications: cholesteric prepolymers}

When chain extending liquid crystalline prepolymers in the presence of tri- or multifunctional reagents it is possible to produce strong liquid crystalline networks. Such networks have interesting properties for film and coating applications: they are strong and are likely to possess good chemical and thermal resistance. The introduction of a chiral mesogenic monomer in the polymer backbone can induce the cholesteric phase, which can be used to produce brightly colored films without the use of pigments. As the color of the material depends on the microstructure of the material, these materials are potentially useful as sensors.

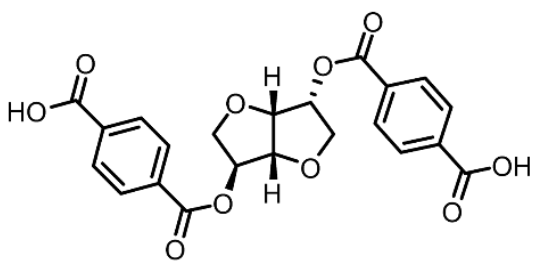

b

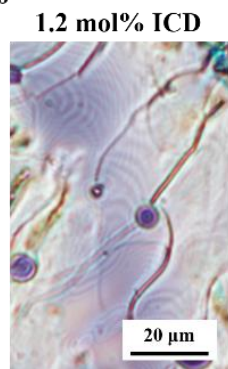

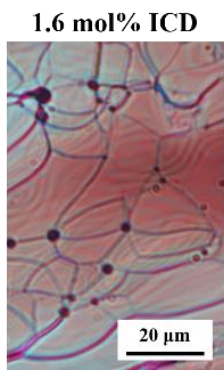

$2.0 \mathrm{~mol} \%$ ICD

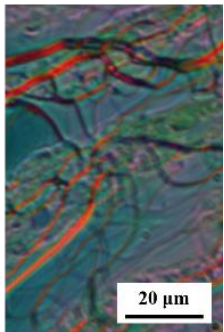

Figure 7.3 (a) Structure of the isosorbide-based chiral dopant (ICD). (b) Cholesteric textures of liquid crystalline prepolymers containing different amounts of ICD, viewed under crossed polarizers at $250^{\circ} \mathrm{C}$.

To explore this possibility an isosorbide-based chiral dopant (ICD, Figure 7.3a) was synthesized and subsequently incorporated in different liquid crystalline prepolymers. The synthesis procedure and monomers used closely resemble those of the other prepolymers described in this thesis. The introduction of the chiral monomer resulted in a distinct change of the observed texture in polarized optical microscopy. In the absence of ICD, nematic Schlieren textures were observed. However, when ICD was 
built into the polymer backbone, cholesteric fingerprint or oily streak textures are observed (Figure 7.3b).

The cholesteric prepolymers had a pearlescent appearance and reflected a range of wavelengths of light dependent on the ICD concentration, resulting in brightly colored films and pieces of material (Figure 7.4a). Conform theory an increased amount of ICD in the polymer resulted in a decrease in the pitch length and correspondingly a decrease in the wavelength of light that was reflected (Figure 7.4b). Interestingly, upon solvent casting the films were initially colorless. Upon heating the chains were able to rearrange within one minute, yielding brightly colored cholesteric films. Deformation of the molten films resulted in the disappearance of the color, due to the disruption of the helical structure. The helical structure quickly reforms while in rest, restoring the films color. In essence, this very simple observation describes a basic mechanical sensing device, displaying the potential of these materials. The different cholesteric prepolymers are miscible, because of the similarity of the chain compositions. This allows mixing of different polymers to tune the pitch and reflected wavelength of the material, which provides a simple route to obtain a broader range of colored films, as shown in Figure 7.4c.

a

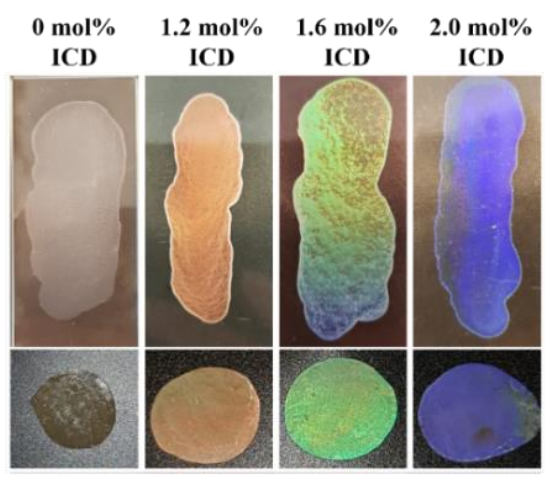

b

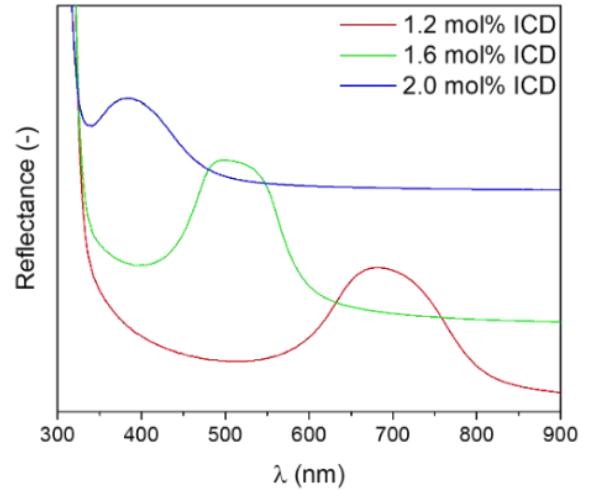

$\mathbf{c}$ Films cast from a mixture of solutions of polymers containing $1.2 \mathrm{~mol} \% \mathrm{ICD}$ and $2.0 \mathrm{~mol} \% \mathrm{ICD}$

$75 \%-25 \%$

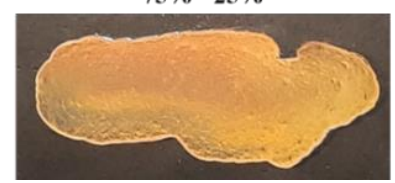

$\mathbf{5 0} \%$ - $\mathbf{5 0} \%$

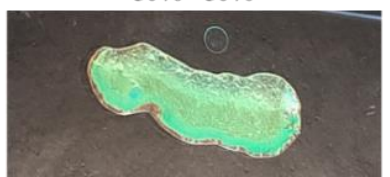

$25 \%-75 \%$

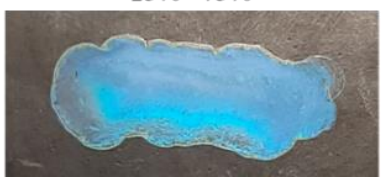

Figure 7.4 (a) Photographs of solvent cast films (top) and melt-pressed pieces (bottom) of cholesteric liquid crystalline prepolymers containing different amounts of ICD. (b) Reflectance as a function of wavelength of solvent cast films of cholesteric liquid crystalline prepolymers. (c) Photographs of solvent cast films. The color was varied by mixing different amount of solutions containing the 1.2 mol\% ICD prepolymer and the 2.0 mol\% ICD prepolymer. 


\subsection{Closing remarks}

I sincerely hope that I have been able to show to you, reader of this thesis, that the knowledge gained and the materials developed have the potential to contribute to the more sustainable use of plastic materials in the future and that these materials provide an interesting starting point for further study on account of their versatility and properties. However, regardless of how interesting they are or how excellent their performance, these are not going to solve the problem that we, as humanity, face regarding the sustainable use of plastic materials and other resources. As a concluding statement, I wish to convey to you, dearest reader, the following message: If we truly wish to create a sustainable future, a bright future for the coming generations, we have to face the possibility that we need to change our ways in a drastic manner. Although much remains be gained by the work of scientists, their efforts need to be supported by both industry and society to have the required impact. It is unfair to rely exclusively on the work of scientists and the development of new technologies to solve our problems regarding sustainability. It is unfair, as all of us have helped create these problems and contributed to them. We must start considering the possibility that we need to change the way we utilize our resources, the way we approach materials and energy, the way we consume, and maybe most importantly the way we desire growth. For generations we have sought prosperity through growth, but growth can never be limitless in a system that knows boundaries. As the growth of a fish is limited by the size of the bowl in which it swims, so humanities growth is limited by the boundaries of our planet. Let us strive to respect those boundaries until we are ready to overcome them. Thank you. 


\section{Summary}

Fiber-reinforced composites, characteristically having high specific stiffness and strength, are interesting as a more sustainable solution (compared to for example metals) in various applications. However, upon end-of-life, recycling of fiber-reinforced composites is challenging. For example, thermomechanical recycling of glass fiberreinforced composites results in deterioration of the mechanical performance, as breaking of the glass fibers during the process decreases the aspect ratio of the fibers. Moreover, processes required for the recovery of the reinforcing phase are energy intensive and the matrix phase is often lost. Thermoplastic LCP reinforced composites, on the other hand, are melt processable and, in theory, provide an opportunity for recycling without loss of mechanical performance. Mechanical performance of the thermoplastic composites is found to be strongly dependent on the morphology that is formed due to flow fields during melt processing. However, variations in the melt viscosity of the different components at a specific shear rate impose a challenge in the reproducibility of the morphology (and the mechanical performance) on recycling. In the past, liquid crystalline polymers (LCPs) have been used to produce reinforced thermoplastic composites, as LCPs are more easily oriented upon flow then regular polymers. However, reproducibility of the LCP based composites properties upon recycling and reprocessing has not yet been assessed.

In this thesis, the potential of LCP reinforced thermoplastic composites is explored with the aim to identify important parameters for the production of reprocessable thermoplastic composites. For the study thermoplastic composite consisting of thermotropic polyesters and polylactide (PLA) is investigated. The study focusses on improving our understanding of 1) the formation and relaxation of interchain orientation of LCPs under deformation, 2) the formation of the morphology during melt processing, and 3) the role of the thermal- and viscoelastic behavior of the blends components in these processes. This knowledge is highly relevant when developing suitable LCP - PLA systems for the production of recyclable LCP reinforced thermoplastic composites.

The processes governing the molecular alignment and relaxation of an aromaticaliphatic thermotropic polyester under shear flow has been followed using a combination of in situ polarized microscopy and in situ Wide-angle X-ray Diffraction (Chapter 2). The domain texture of the LCP initially stretches upon startup of the shear flow with a simultaneous increase in interchain orientation. Subsequently the polydomain texture breaks up at higher degrees of deformation, while the degree of interchain orientation remains constant. Though the polydomain texture of LCPs is 
influenced by the applied shear rate, the final degree of interchain orientation is independent of the shear rate. During relaxation, the stretched domains of the LCP first contract and subsequently coalesce. The relaxation of the LCP orientation does not start instantaneously and only decreases upon coalescence of the LCP domains. The relaxation of orientation is faster when a finer texture is formed (i.e. when higher shear rates are applied), and this interdependence will have significant implications for the processing of LCPs and LCP-polymer composites.

The morphological development of two different LCPs in a PLA matrix in different processing steps is characterized in detail, and is interpreted within a theoretical framework based on the capillary number $(x)$ and the viscosity ratio $(\lambda)$ (Chapter 3 ). The use of LCPs with a lower viscosity and concomitantly a lower $\lambda$ results a finer dispersion, but also results in accelerated relaxation of the LCP domains within the matrix. Crystallization of the LCP, resulting in a sudden increase in $\lambda$ that prevents droplet deformation, negatively affects the reinforcement in an injection molding process. LCPs that are amorphous in nature typically do not show sudden increase in $\lambda$. Therefore, compared to semi-crystalline LCPs, amorphous LCPs are likely to be more suitable as the dispersed phase in an injection molding process. Extensional flow, such as experienced in melt spinning, is found to be highly efficient in the formation of LCP fibrils, regardless of the viscosity ratio between the LCP and polymer matrix (PLA). In shear flow, on the other hand, deformation of droplets is only possible for viscosity ratios below 3.8.

The effect of the molecular weight of the PLA matrix on the morphology and the performance of the composites is studied using a commercially available LCP and several different PLA matrices (Chapter 4). Two distinct regimes, dependent on the LCP/PLA viscosity ratio are identified: In the first regime $(\lambda<1)$, a higher matrix viscosity results in a slightly coarser morphology. However, the overall mechanical performance is not influenced as the LCP retains a fibrillar morphology, and the degree of molecular orientation of the LCP remains high. In the second regime $(\lambda>1)$, a lower matrix viscosity results in a less effective deformation of the LCP droplets during processing. When the viscosity ratio exceeds a value of 3.8 the LCP droplets can no longer be deformed by the matrix: the application of shear flow results in a nodular LCP morphology. In the second regime, the mechanical performance of the thermoplastic composite decreases with decreasing molecular weight of the matrix. These two regimes are retained upon repeated processing (thermomechanical recycling) of the composite materials. However, changes in morphology and performance are observed because of the decrease in the PLA viscosity due to the decreasing molecular weight of the PLA with the number of reprocessing cycles. Reprocessing of LCP reinforced thermoplastic 
composites, without any loss of mechanical performance could be achieved under the condition that the viscosity of the PLA matrix remains sufficiently high to satisfy the condition of $\lambda<1$.

In Chapter 4 of this thesis, the viscosity ratio of the blend is identified as a key parameter for recyclability, whereas Chapter 3 shows that an amorphous LCP is to be preferred over a semi-crystalline LCP. This suggests that improvements in the reprocessability of composites can be made by selecting LCPs with a low processing temperature and a tunable viscosity. Such LCPs are presented in this thesis (Chapter 5): control over the LCP viscosity is achieved via a chain extension reaction of an amorphous liquid crystalline prepolymer using bis(2-oxazoline)s as coupling agents. The chain extension can be easily carried out via reactive extrusion, and molecular weight can be controlled via the molar ratio of liquid crystalline prepolymer to bis(2-oxazoline). The resulting LCPs are found to have excellent mechanical properties. With the increase in the molecular weight of the LCP, a transition in the viscoelastic behavior from an unentangled nematic melt to a constrained melt is observed. Interestingly, in the constrained melt, a minimal loss in the orientation parameter is observed even upon heating the chain-extended LCPs to temperatures well above their glass transition temperature.

To assess the effect of the LCP viscosity on the morphology in more detail, composites of the new LCPs (as reported in Chapter 5) with PLA have been produced, and the reprocessability of the LCP-PLLA composites is demonstrated (Chapter 6). In accordance with their viscosity ratios, a suitable fibrillar morphology is obtained via injection molding for all composites, even forming fibrils with sub-micron diameters in the case of the LCPs with lower molar mass. Effective reinforcement is achieved and the stiffness and strength of the thermoplastic composites increased when using LCPs with a higher viscosity. Interestingly, the expected decrease in strain at break that typically accompanies polymer matrix reinforcement is not observed when using LCPs with higher molar masses $\left(M_{\mathrm{w}}>40 \mathrm{~kg} \mathrm{~mol}^{-1}\right.$ according to GPC analysis). The relaxation of the interchain orientation of the LCP in a PLA matrix is evaluated in detail via in situ Wide-angle X-ray Diffraction. The viscosity of the matrix phase is found to be the dominant parameter. In blends, the mechanism by which the interchain orientation of the LCP decreases differs from that of LCPs without matrix. It is related to the relaxation of the fibrillar LCP morphology within the PLA melt and is affected by the stereoregularity and crystallinity of the matrix. The presence of the LCP is found to affect the cold crystallization of the PLLA matrix. Although no increase in nucleation or crystallization rate is observed, the orientation and growth of the PLLA crystallites is affected. 
In this thesis, the potential of LCP reinforced thermoplastic composites is explored with the aim to identify important parameters for the production of reprocessable thermoplastic composites. The thesis conclusively demonstrates that for the combination of a thermotropic polyester and PLA, it is feasible to use amorphous LCPs with a low processing temperature. The use of a suiltable LCP (viscosity slightly lower than the molten matrix, amorphous) allows the formation of the required fibrillar morphology under different processing conditions, and simultaneously limits the thermal degradation of the PLA matrix phase. Recycling of LCP-PLA composites without loss of mechanical performance is demonstrated, under the condition that the viscosity ratio remains below one. Based on these findings, a system is developed to produce amorphous LCPs with a tunable viscosity. The effectiveness of the LCPs in composites with PLA is demonstrated. These finding, investigated in a specific system, are of a generic nature and can therefore be translated to other polymer-polymer system. 


\section{Acknowledgements}

The past four years have been full of interesting discussions, exciting adventures and most importantly full of people without whom the road and the result would have turned out quite different.

First, I would like to thank Karel and Sanjay, for their supervision, their input and their help. It has been a pleasure to work with you and I wish you all the best in your new endeavors. Sanjay, thank you for the guidance. I am happy you can enjoy being a scientist again in KAUST and I hope I get the opportunity to visit you in the future. Karel, bedankt voor alle hulp. Je zat altijd vol goede ideeën en had steeds het overzicht. Gedurende mijn PhD is niet alles gelopen zoals gepland, maar het is gelukt! Er is een mooie thesis gekomen en we hebben samen veel geleerd. Bedankt dat je me de kans hebt gegeven om een gast college te geven en ik vond het erg gaaf dat je me aan de studenten introduceerde onder mijn bijnaam (Goose). Als je op Chemelot bent en je zin hebt in een kop koffie dan weet je me te vinden.

My thanks goes out to Lorenzo Moroni, Andrij Pich, Sijbrand van der Zwaag and Dimitros Vlassopoulos. Thank you for taking the time to go through my thesis and provide your feedback. Your contributions have greatly improved the result.

I would like to thank Benny, Mark and Dietmar, not so much for your contributions to my $\mathrm{PhD}$, but for helping me explore my interests, encouraging my curiosity and helping me learn. Without you, I would not have ended up where I am today. Thank you for guiding a young scientist.

Milo en Lucienne, Roy en Celine, bedankt voor jullie harde werk en jullie vertrouwen. Jullie bijdragen aan deze thesis zijn heel verschillend, maar niet te onderschatten. Ik ben heel blij dat ik met jullie heb mogen werken. Jullie maakten mijn werk zoveel leuker, interessanter en gezelliger. Ik vind het heel gaaf dat jullie allemaal nog even zijn blijven hangen om een master of een $\mathrm{PhD}$ te doen.

Chemelot itself is not a place with an inherently great atmosphere. However, great colleagues make the difference. Thank you, Martien, Ramiro, Milo, Renato, Ravi, Nils, Lawrence, Dario, Carlos, Vahid, Monika, Anne, Feiji, Huaqin, Jules, Mohan, Pouya, Jurrie, Ola, Marie, Christian, Ele and Lilly. It has been great to talk, laugh, have coffee and drinks, work and play football with you. 
Sometimes colleagues turn into great friends. Geert, Varun, Andrea and Manta, we have shared so much the past years. We went through utter misery and absolute joy but most importantly, we went through it together. I will forever remember the holidays, conferences, (exceptionally long) coffee breaks, experiments, games, dinners, discussions, drinks, barbecues, and other shenanigans. We did too much fun stuff to mention here, but I am just going to list a few more: we played table soccer and pool (angles!), had big Indian lunches, created and maintained an office jungle (and the necessary trips to Intratuin) and we went all out on Christmas decorations (including sweaters and hats). It has been a preposterous pleasure to share an office with you (it really isn't the same without you). Thank you for becoming and remaining great friends. Let's stay in touch.

Het kan lastig zijn om je gezond verstand te behouden in stressvolle periodes, maar dankzij jullie is het mij in ieder geval gelukt. Bedankt Gilles, Ellen, Annelies, Marijne, Bianca, Bea, Sander, Remco, Tom, Rick, Jan Willem, Harm en Akira voor de mooie momenten: even een drankje doen (wat enorm uit de klauwen loop), uit eten, bruiloften, op vakantie (zowel ver weg als minder ver weg), weekendjes erop uit en verschillende feestjes (voor allerlei verschillende redenen). Veel van deze momenten hebben of verdienen een eigen verhaal maar het zijn er te veel om op te noemen.

Lieve familie, Pap, Mam en Bente. Bedankt voor jullie steun, ik kon altijd bij jullie terecht als ik er even uit moest of ruimte nodig had.

Sarah, j'ai eu la chance de te rencontrer pendant mon doctorat. Immédiatement, nous sommes devenus amis et maintenant nous sommes plus. Tu as amélioré ma vie et j’en suis certain tu continueras à le faire dans l'avenir. Merci.

Thank you all,

Gijs / Goose

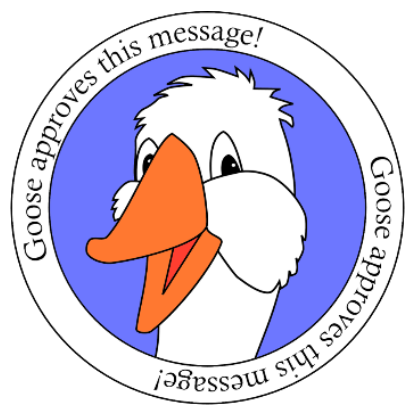




\section{Curriculum Vitae}

Gijsbert Willem de Kort (Gijs) was born in Waalwijk on July 30, 1992. In 2010, he graduated from the Theresialyceum in Tilburg and he subsequently started his academic career at the Eindhoven University of Technology. There he received a BSc (2013) and MSc (2015) in Chemical Engineering. During his studies, he developed an interest in polymeric materials and finding way to make them more sustainable. His bachelor thesis was focused on recycled poly(propylene). The topic of his master thesis was the synthesis and characterization of poly(pentadecalactone) with increased melt strength. During his studies, he did an industrial internship at DSM Coating Resins in Zwolle, where he worked on crystallization of powdercoating resins. He started his $\mathrm{PhD}$ in 2016 at the Aachen Maastricht Institute for Biobased Materials (Maastricht University) on the topic of reinforced thermoplastic composites consisting of LCPs and PLA. During this period, he passed the RPK courses A to D (2016-2018), published papers on the work described in this thesis and took part in collaborations, both within and outside of AMIBM. Currently, he remains at AMIBM as a postdoctoral researcher on the topic of sustainable engineering plastics. 


\section{List of publications}

\section{Patent:}

- $\quad$ de Kort, G. W.; Wilsens, C. H. R. M.; Rastogi, S., High Performance LCP Materials (Universiteit Maastricht) PCT/EP2020/068764.

\section{Publications:}

- $\quad$ de Kort, G. W.; Leoné, N.; Stellamanns, E.; Auhl, D.; Wilsens, C. H. R. M.; Rastogi, S. Effect of Shear Rate on the Orientation and Relaxation of a Vanillic Acid Based Liquid Crystalline Polymer. Polymers. 2018, 10, 935.

- de Kort, G. W.; Rastogi, S.; Wilsens, C. H. R. M. Controlling Processing, Morphology, and Mechanical Performance in Blends of Polylactide and Thermotropic Polyesters. Macromolecules, 2019, 52, 6005-6017.

- de Kort, G. W.; Bouvrie, L.; Rastogi, S.; Wilsens, C. H. R. M. Thermoplastic PLALCP Composites: A Route towards Sustainable, Reprocessable, and Recyclable Reinforced Materials. Sustain. Chem. Eng. 2020, 8, 624-631.

- de Kort, G. W.; Saidi, S.; Hermida-merino, D.; Leoné, N.; Srinivas, V.; Rastogi, S.; Wilsens, C. H. R. M. Importance of Viscosity Control for Recyclable Reinforced Thermoplastic Composites. Macromolecules, 2020, 53, 6690-6702.

- $\quad$ de Kort, G. W.; Saidi, S.; Hermida-merino, D.; Leoné, N.; Rastogi, S.; Wilsens, C. H. R. M. A Reactive Processing Route to Thermotropic Polyesters with Low Processing Temperature and Enhanced Relaxation Time., Macromolecules, 2020 (Under review).

\section{Unrelated to this thesis:}

- Jia, M.; Zhang, D.; De Kort, G. W.; Wilsens, C. H. R. M.; Rastogi, S.; Hadjichristidis, N.; Gnanou, Y.; Feng, X. All-Polycarbonate Thermoplastic Elastomers Based on Triblock Copolymers Derived from Triethylborane-Mediated Sequential Copolymerization of $\mathrm{CO}_{2}$ with Various Epoxides. Macromolecules, 2020, 53 (13), 5297-5307

- Appels, F. V. W.; van den Brandhof, J. G.; Dijksterhuis, J.; de Kort, G. W.; Wösten, H. A. B. Fungal Mycelium Classified in Different Material Families Based on Glycerol Treatment. Commun. Biol. 2020, 3 (1) 
- Roy, M.; Zhelezniakov, M.; de Kort, G. W.; Hawke, L. G. D.; Leoné, N.; Rastogi, S.; Wilsens, C. H. R. M. On the Nucleation of Polylactide by Melt-Soluble Oxalamide Based Organic Compounds. Polymer 2020, 202

- Wilsens, C. H. R. M.; Hawke, L. G. D.; De Kort, G. W.; Saidi, S.; Roy, M.; Leoné, N.; Hermida-Merino, D.; Peters, G. W. M.; Rastogi, S. Effect of Thermal History and Shear on the Viscoelastic Response of IPP Containing an Oxalamide-Based Organic Compound. Macromolecules 2019, 52 (7), 2789-2802

- Leoné, N.; Roy, M.; Saidi, S.; De Kort, G.; Hermida-Merino, D.; Wilsens, C. H. R. M. Improving Processing, Crystallization, and Performance of Poly-1-Lactide with an Amide-Based Organic Compound as Both Plasticizer and Nucleating Agent. ACS Omega 2019, 4 (6)

- Wilsens, C. H. R. M.; Hawke, L. G. D.; Troisi, E. M.; Hermida-Merino, D.; De Kort, G.; Leoné, N.; Saralidze, K.; Peters, G. W. M.; Rastogi, S. Effect of SelfAssembly of Oxalamide Based Organic Compounds on Melt Behavior, Nucleation, and Crystallization of Isotactic Polypropylene. Macromolecules 2018, 51 (13), 48824895 